
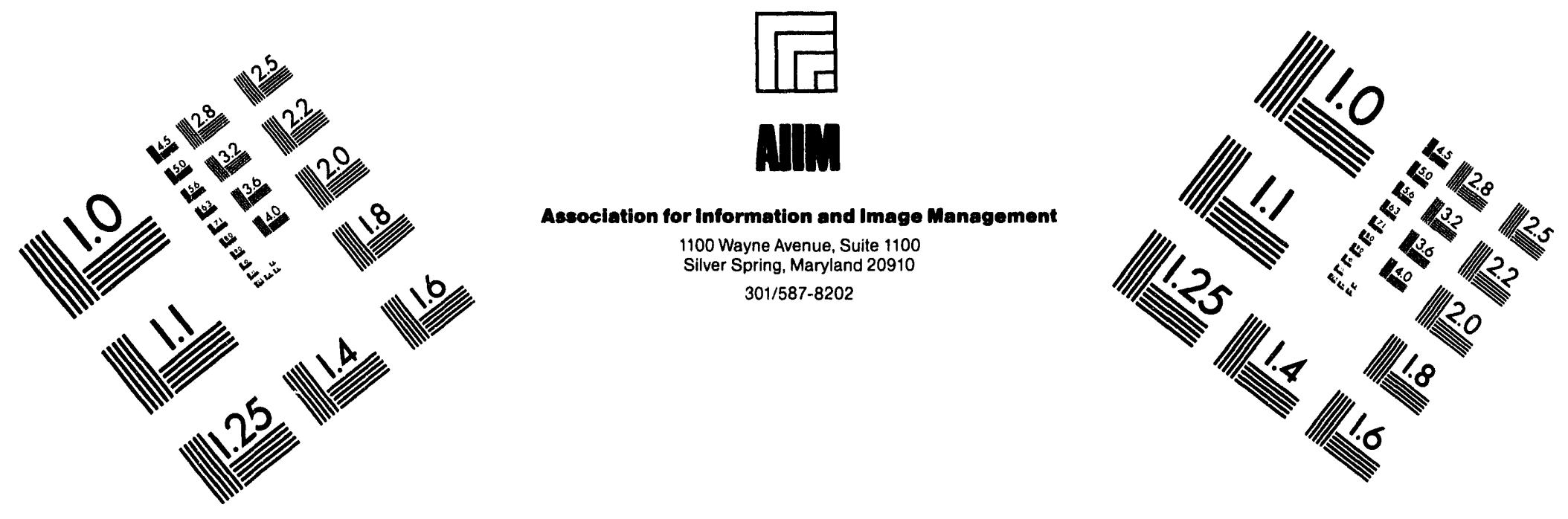

\title{
Centimeter
}

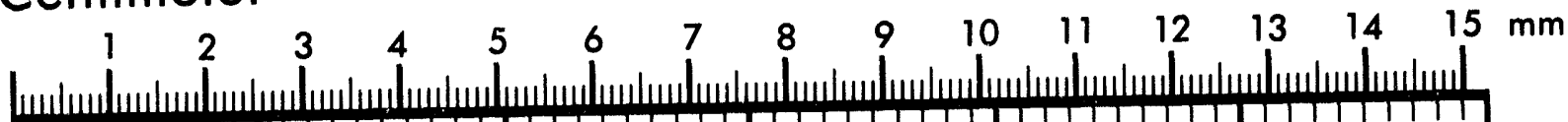

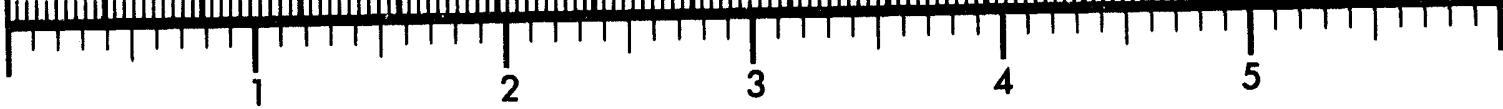
Inches
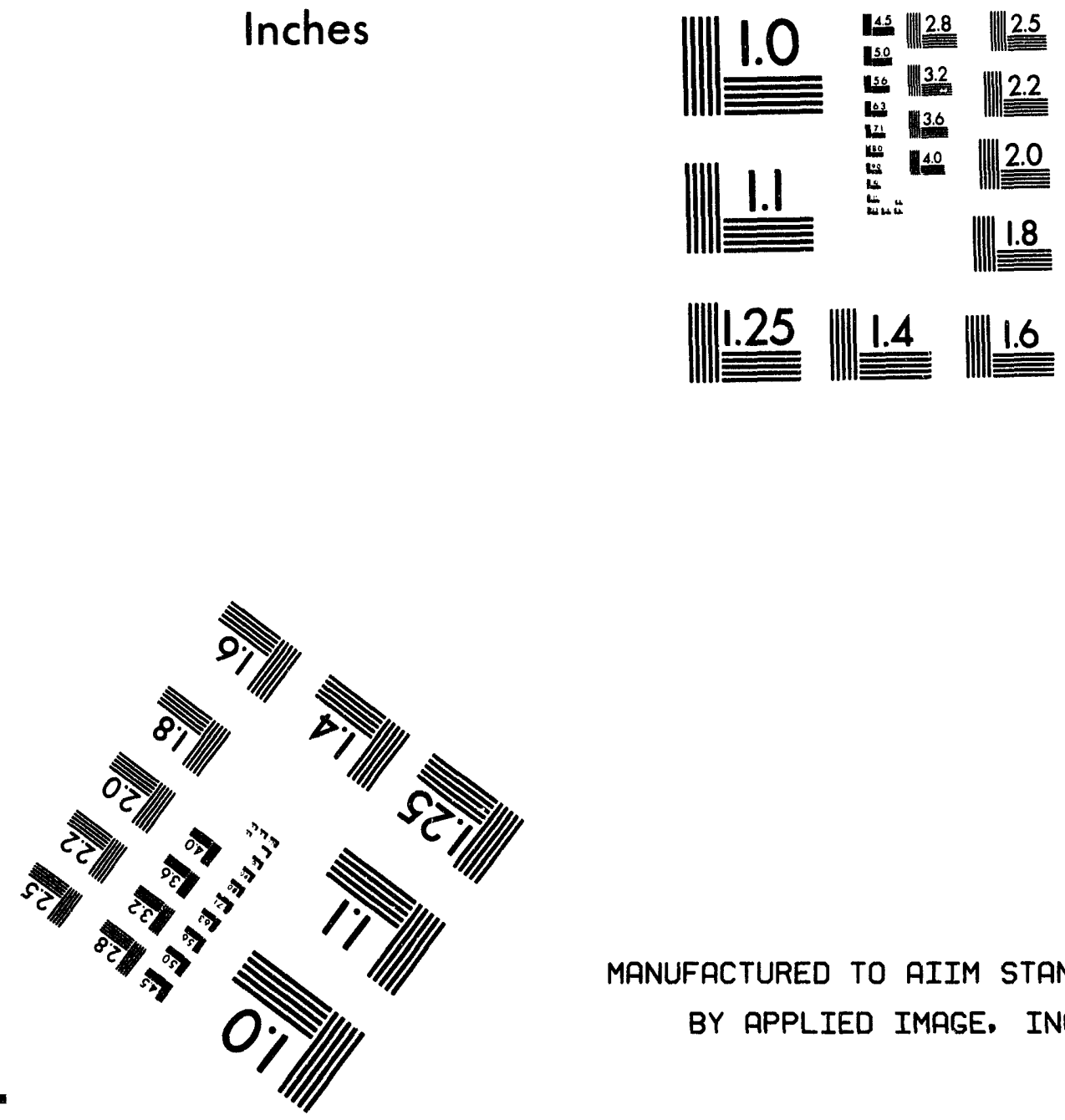

MANUFACTURED TO AIIM STANDARDS

$$
\text { BY APPLIED IMAGE, INC. }
$$

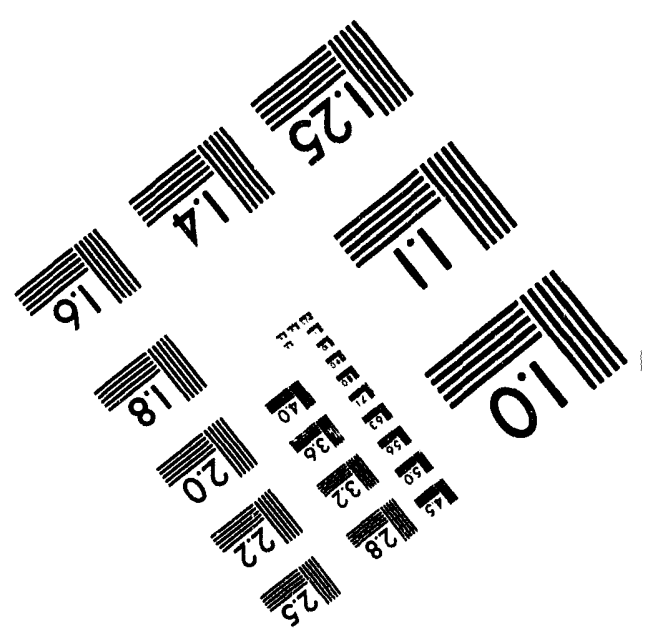



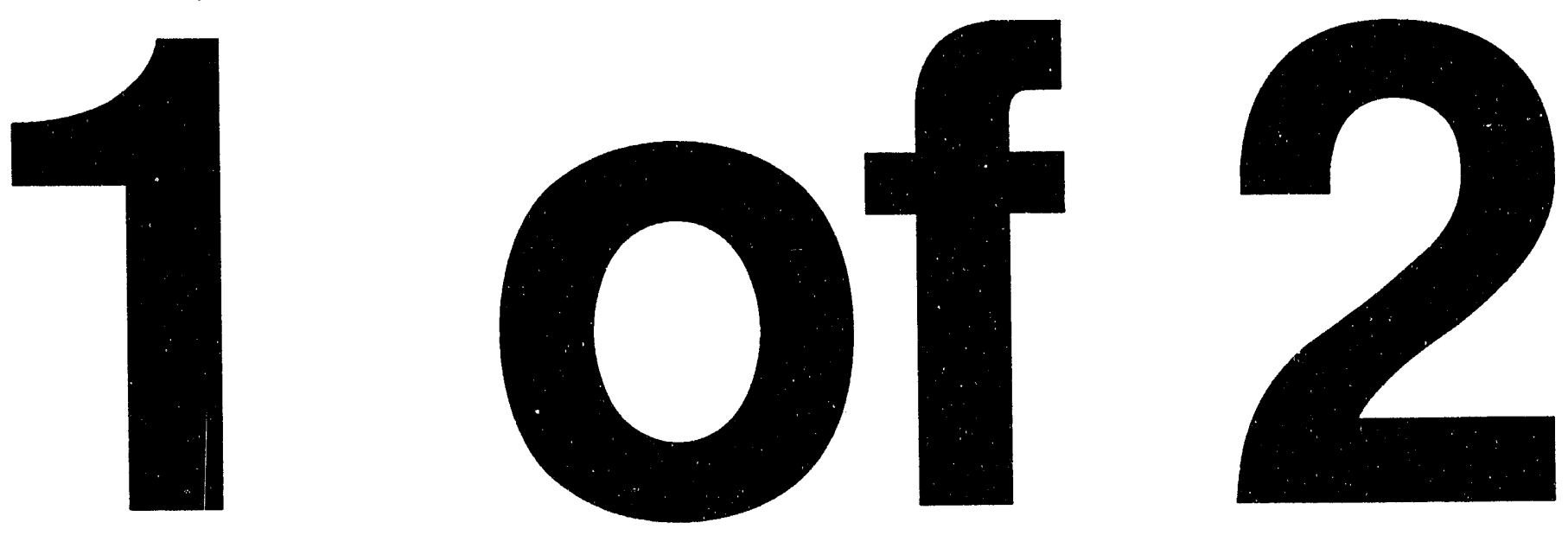


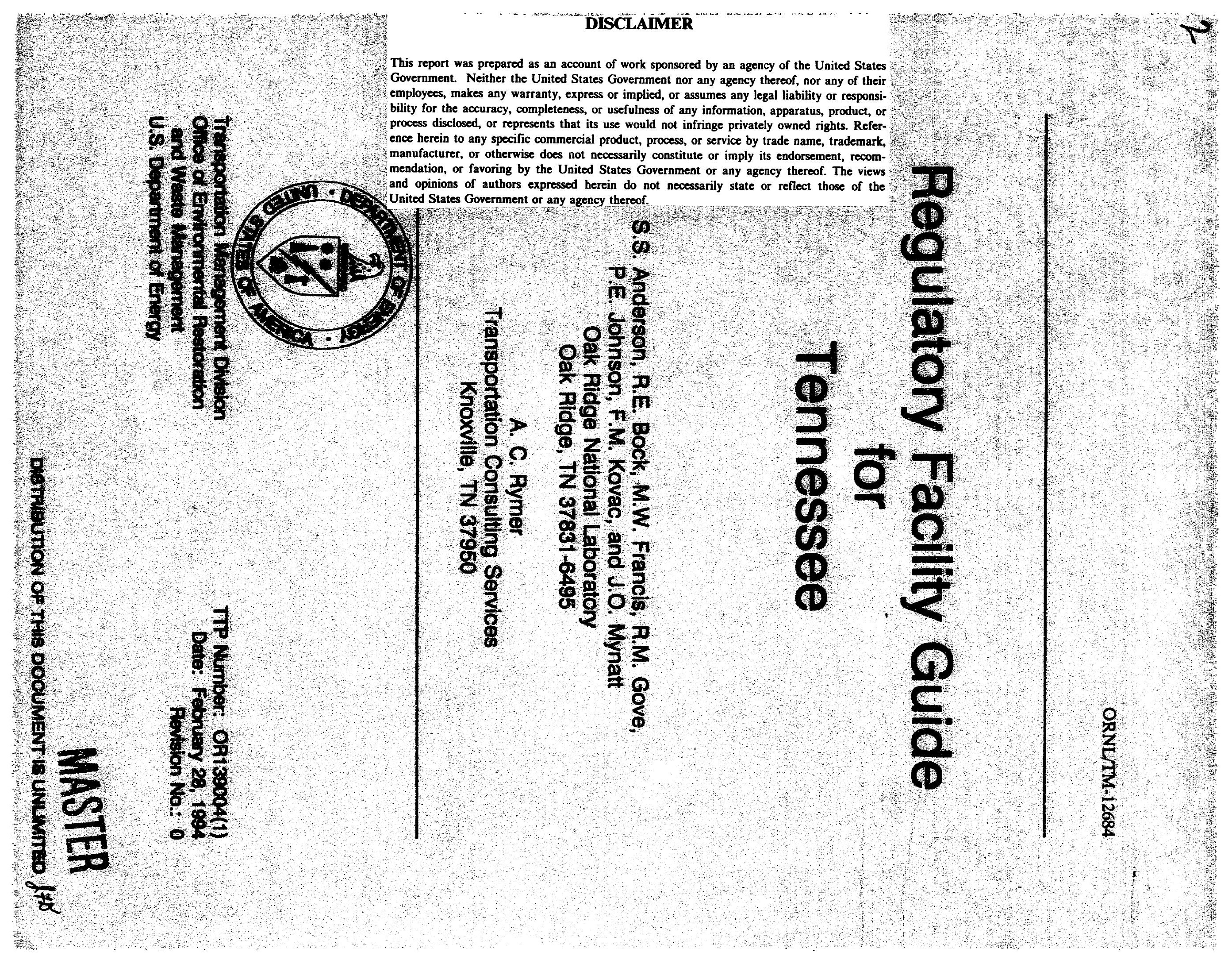




\section{REGULATORY FACILITY GUIDE FOR TENNESSEE}

ASSIGNED TO:-OSTI

COPY NUMBER:_ $\quad 119$

You are charged with responsibility for this copy of ORNL/TM-12684, entitled Regulatory Facility Guide for Tennessee. If new or revised pages are issued, you are responsible for inserting the new pages and destruction of superseded pages.

If this report is reassigned or is no longer needed, please report the name and address of the new assignee or return the report to:

J.O. Mynatt

Oak Ridge National Laboratory

Building 2001, MS-6050

Oak Ridge, TN 37831-6050 
ORNL/TM-12683

Revision 0

Date: February 1994

\section{REGULATORY FACIIITY GUIDE FOR TENNESSEE} PAGE CONTROL SHEET

Title Page

Acknowledgements

Table of Contents

Table of Contents (continued)

Table of Contents (continued)

Introduction

viii Introduction (continued)

ix Acronyms

1-1 1.1 Shipper Activities

1-2 1.1 Shipper Activities (continued)

1.3 1.1 Shipper Activities (continued)

1-4 1.1 Shipper Activities (continued)

1.5 1.2 Carrier Activities

1.6 1.2 Carrier Activities (continued)

1.7 1.2 Carrier Activities (continued)

1-8 1.3 Receiver Activities

Rev. 0, 02/94

Rev. 0, 02/94

Rev. 0, 02/94

Rev. 0, 02/94

Rev. 0, 02/94

Rev. 0, 02/94

Rev. 0, 02/94

Rev. 0, 02/94

Rev. 0, 02/94

Rev. 0, 02/94

Rev. 0, 02/94

Rev. 0, 02/94

Rev. 0, 02/94

Rev. 0, 02/94

Rev. 0, 02/94

Rev. 0, 02/94

2-1 2.1 Shipper Operations Jurisdiction

2-2 2.1 Shipper Operations Jurisdiction (continued)

2-3 2.1 Shipper Operations Jurisdiction (continued)

2-4 2.1 Shipper Operations Jurisdiction (continued)

2-5 2.1 Shipper Operations Jurisdiction (continued)

2-6 2.1 Shipper Operations Jurisdiction (continued)

2-7 2.2 Carrier Operations Jurisdiction

2-8 2.2 Carrier Operations Jurisdiction (continued)

2.9 2.2 Carrier Operations Jurisdiction (continued)

Rev. 0, 02/94

Rev. 0, 02/94

R.v. 0, 02/94

Rev. 0, 02/94

Rev. 0, 02/94

Rev. 0, 02/94

Rev. 0, 02/94

Rev. 0, 02/94

Rev. 0, 02/94

3-1 3.1 State Transit Table

3-2 3.1 State Transit Table (continued)

3-3 3.1 State Transit Table (continued)

3-4 3.1 State Transit Table (continued)

3.5 3.1 State Transit Table (continued)

3-6 3.1 State Transit Table (continued)

3-7 3.1 State Transit Table (continued)

3.8 3.1 State Transit Table (continued)

3-9 3.1 State Transit Table (continued)

3-10 3.2 Tribal Transit Table

Rev. 0, 02/94

Rev. 0, 02/94

Rev. 0, 02/94

Rev. 0, 02/94

Rev. 0, 02/94

Rev. 0, 02/94

Rev. 0, 02/94

Rev. 0, 02/94

Rev. 0, 02/94

Rev. 0, 02/94

Rev. 0, 02/94

Rev. 0, 02/94

Appendix A - References

Appendix B - Directory of State Transportation Agencies and Senior Officials

Appendix C - Import/Export Information and Requirements

Rev. 0, 02/94

Rev. 0, 02/94 


\section{Regulatory Facility Guide for \\ Tennessee}

S.S. Anderson, R.E. Bock, M.W. Francis, R.M. Gove, P.E. Johnson, F.M. Kovac, and J.O. Mynatt

Oak Ridge National Laboratory

Oak Ridge, TN 37831-6495

A. C. Rymer

Transportation Consulting Services

Knoxville, TN 37950

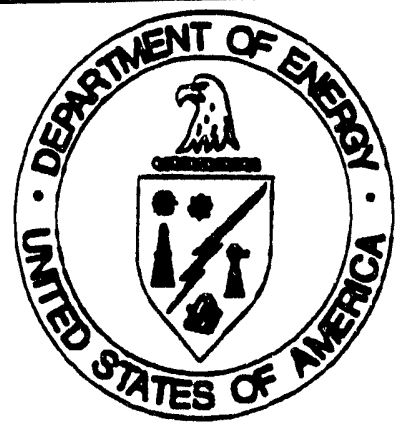

Transportation Management Division Office of Environmental Restoration and Waste Management U.S. Department of Energy
TTP Number: OR139004(1) Date: February 28, 1994 Revision No.: 0 
This report was prepared as an account of work sponsored by an agency of the United States Government. Neither the United States Government nor any agency thereof, nor any of their employees, makes any warranty, express or implied, or assumes any legal liability or responsibility for the accuracy, completeness, or usefulness of any information, apparatus, product, or process disclosed, or represents that its use would not infringe privately owned rights. Reference herein to any specific commercial product, process, or service by trade name, trademark, manufacturer, or otherwise, does not necessarily constitute or imply its endorsement, recommendation, or favoring by the United States Government or any agency thereof. The views and opinions of authors expressed herein do not necessarily state or retlect those of the United States Government or any agency thereot. 


\section{ACKNOWLEDGEMENTS}

The authors gratefully acknowledge the help of the numerous individuals who contributed to preparing this guide, especially:

\section{Battelle Seattle Research Center}

Marcus K. Lester

Edward E. Liebow

\section{Oak Ridge National Laboratory}

Ronald B. Pope

Joseph E. Ratledge

Julia M. Stockstill 
.

-

- 


\section{TABLE OF CONTENTS}

Page

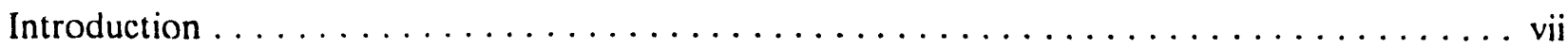

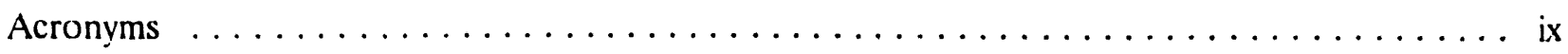

1. Part 1 - RFG Summary Tables

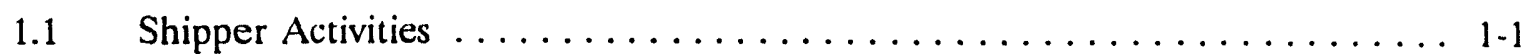

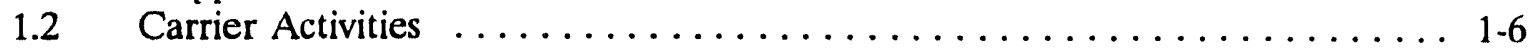

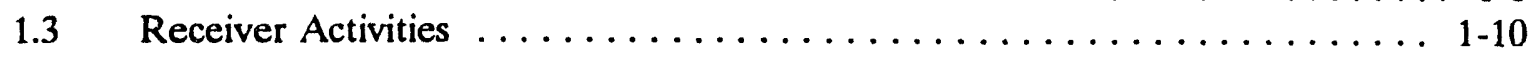

2. Part 2 - Operations Jurisdiction Tables

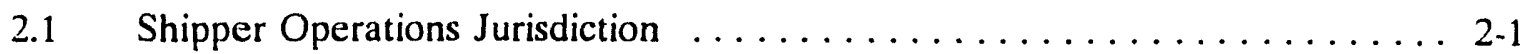

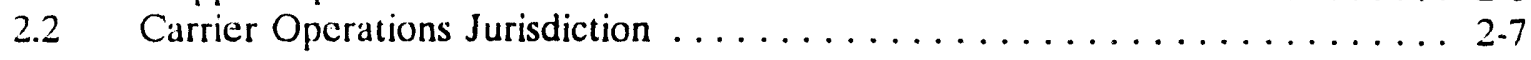

3. Part 3 - RFG Transit Tables

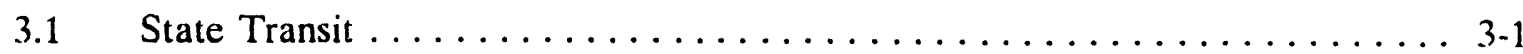

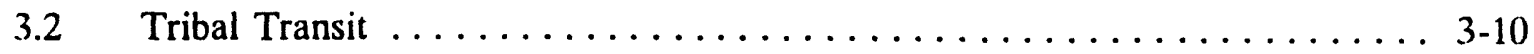

4. Part 4 - RFG Preferred Routes and Maps

4.1 Alabama Preferred Routes

4.1.1 Alabama Preferred Routes Map

4.2 Arizona Preferred Routes

4.2.1 Arizona Preferred Routes Map

4.3 Arkansas Preferred Routes

4.3.1 Arkansas Preferred Routes Map

4.4 California Preterred Routes

4.4.1 California Preferred Routes Map

4.4.2 Southern California Preferred Routes Map

4.4.3 Northern California Preferred Routes Map

4.5 Colorado Preferred Routes

4.5.1 Colorado Preferred Routes Map

4.5.2 Colorado (Denver and vicinity) Preferred Routes Map

4.6 Connecticut Preferred Routes
4.6.1 Connecticut Preferred Routes Map

4.7 Delaware Preferred Routes

4.7.1 Delaware Preferred Routes Map

4.8 District of Columbia Preferred Routes

4.9 Florida Preferred Routes

4.9.1 Florida Preferred Routes Map

4.10 Georgia Preferred Routes

4.10.1 Georgia Preferred Routes Map 


\section{TABLE OF CONTENTS (Continued)}

4.11 Idaho Preferred Routes

4.11.1 Idaho Preferred Routes Map

4.12 Illinois Preferred Routes

4.12.1 Illinois Preferred Routes Map

4.13 Indiana Preferred Routes

4.13.1 Indiana Preferred Routes Map

4.14 Iowa Preferred Routes

4.14.1 Iowa Preferred Routes Map

4.15 Kansas Preferred Routes

4.15.1 Kansas Preferred Routes Map

4.16 Kentucky Preferred Routes

4.16.1 Kentucky Preferred Routes Map

4.17 Louisiana Preferred Routes

4.17.1 Louisiana Preferred Routes Map

4.18 Maine Preferred Routes

4.18.1 Maine Preferred Routes Map

4.19 Maryland Preferred Routes

4.19.1 Maryland Preferred Routes Map

4.20 Massachusetts Preferred Routes

4.20.1 Massachusetts Preferred Routes Map

4.21 Michigan Preferred Routes

4.21.1 Michigan Preferred Routes Map

4.22 Minnesota Preferred Routes

4.22.1 Minnesota Preferred Routes Map

4.23 Mississippi Preferred Routes

4.23.1 Mississippi Preterred Routes Map

4.24 Missouri Preferred Routes

4.24.1 Missouri Preferred Routes Map

4.25 Montana Preferred Routes

4.25.1 Montana Preferred Routes Map

4.26 Nebraska Preferred Routes

4.26.1 Nebraska Preferred Routes Map

4.27 Nevada Preferred Routes

4.27.1 Nevada Preferred Routes Map

4.28 New Hampshire Preferred Routes

4.28.1 New Hampshire Preferred Routes Map

4.29 New Jersey Preferred Routes

4.29.1 New Jersey Preferred Routes Map

4.30 New Mexico Preferred Routes

4.30.1 New Mexico Preferred Routes Map

4.31 New York Preferred Routes

4.31.1 New York Preferred Routes Map 


\section{TABLE OF CONTENTS (Continued)}

4.32 North Carolina Preferred Routes

4.32.1 North Carolina Preferred Routes Map

4.33 North Dakota Preferred Routes

4.33.1 North Dakota Preferred Routes Map

4.34 Ohio Preferred Routes

4.34.1 Ohio Preferred Routes Map

4.35 Oklahoma Preferred Routes

4.35.1 Oklahoma Preferred Routes Map

4.36 Oregon Preferred Routes

4.36.1 Oregon Preferred Routes Map

4.37 Pennsylvania Preferred Routes

4.37.1 Pennsylvania Preferred Routes Map

4.38 Rhode Island Preferred Routes

4.38.1 Rhode Island Preferred Routes Map

4.39 South Carolina Preferred Routes

4.39.1 South Carolina Preferred Routes Map

4.40 South Dakota Preferred Routes

4.40.1 South Dakota Preferred Routes Map

4.41 Tennessee Preferred Routes

4.41.1 Tennessee Preferred Routes Map

4.42 Texas Preferred Routes

4.42.1 Texas Preferred Routes Map

4.42.2 Texas (Dallas-Fort Worth area) Preferred Routes Map

4.43 Utah Preferred Routes

4.43.1 Utah Preferred Routes Map

4.44 Vermont Preferred Routes

4.44.1 Vermont Preferred Routes Map

4.45 Virginia Preferred Routes

4.45.1 Virginia Preferred Routes Map

4.46 Washington Preferred Routes

4.46.1 Washington Preferred Routes Map

4.47 West Virginia Preferred Routes

4.47.1 West Virginia Preferred Routes Map

4.48 Wisconsin Preferred Routes

4.48.1 Wisconsin Preferred Routes Map

4.49 Wyoming Preferred Routes

4.49.1 Wyoming Preferred Routes Map

Appendix A Appendix B Appendix $\mathrm{C}$
References

Contacts Directory

Import/Export Information and Requirements 


\section{Regulatory Facility Guide for Tennessee}

This Regulatory Facility Guide (RFG) has been developed for the U. S. Department of Energy (DOE) and contractor facilities located in the state of Tennessee. It provides detailed compilations of international, federal, and state transportation-related regulations applicable to shipments originating at or destined to Tennessee facilities. This RFG was developed as an additional resource tool for use both by traffic managers who must ensure that transportation operations are in full compliance with all applicable regulatory requirements and by oversight personnel who must verify compliance activities.

This document is a resource tool. It is to be used as an index to guide you through the process of completing the type of shipment required. Its use is not to be substituted for the applicable regulations.

The RFG communicates information by means of summarized tables which guide the user to specific citations pertaining to a variety of transportation operations. The RFG consists of four main parts.

Part 1 contains the RFG Summary Tables for shippers, carriers, and receivers. These summary tables are constructed in a way that quickly points the user to the international, federal, or state citation codes pertaining to each designated activity. In the first table shown, SHIPPER ACTIVITIES, the first activity listed is Carrier Qualifications. As the user looks across the row/page from left to right, the first regulation listed under RSPA (Research and Special Programs Administration) can be found in the Code of Federal Regulations: (CFR), Title 49, between sections 100 and 199. For the specific CFR regulation, look at the 49 CFR 100-199 column directly across from the Carrier Qualifications activity. Therefore. for Carrier Qualifications, the specific CFR reference can be found in 49 CFR 171.1; 49 CFR 171.2(a); 49 CFR 174.7; 49 CFR 175.20; 49 CFR 176.13; and 49 CFR 177.800. Where no regulation requirement has been identified, (e.g. refer to DOC, 15 CFR, 19 CFR for Carrier Qualifications), the column is left blank.

Part 2 consists of Operations Jurisdiction Tables for the shipper and carrier. These jurisdiction tables provide detailed information for specific types of shipments and various carrier operations. They are formatted in the same manner as the activity tables so that the user is guided from the left-hand column which lists the detailed types of shipments across the page to the applicable agency and regulations for those shipments.

Part 3 contains the Transit Tables for states and federally-recognized tribal entities which provide state and tribal information for the states through which the transportation routes 
traverse. It provides identification of offices which need to be notified, telephone numbers, permit requirements, and other pertinent information. The tribal information, which is not complete at this time and will be further developed during FY 1994, will be included in all future updates.

Part 4 consists of "preferred routes" satisfying the 49 CFR 177.825 requirements for the transport of highway route controlled quantities of radioactive materials for each state, including alternative routes designated by the state. In addition, a map of each state (excluding Alaska and Hawaii) which shows the "preferred routes" is also provided.

There are three appendices: Appendix A provides a list of references used in the guide; Appendix B provides a contacts directory; and Appendix C provides import/export information and requirements. There is also an RFG List of Acronyms which appears on page ix of this document.

Since the RFGs are an evolving set of documents; corrections, comments, and suggested additions are solicited. Please provide comments to:

\author{
Stephanie S. Anderson \\ Oak Ridge National Laboratory \\ P.O. Box 2008 \\ Oak Ridge, TN 37831-6495 \\ Phone: (615) 576-8794 \\ FAX: (615) 574-3431
}

(Ms. Anderson has lead responsibility at ORNL for this activity on behalf of DOE's Transportation Management Division) 


\section{List of Acronyms}

$\begin{array}{ll}\text { CFR } & \text { Code of Federal Regulations } \\ \text { CMV } & \text { Commercial Motor Vehicle } \\ \text { DIR } & \text { Regulations are directly applicable to the operation. } \\ \text { DOC } & \text { Department of Commerce } \\ \text { DOE } & \text { Department of Energy } \\ \text { DOT } & \text { Department of Transportation } \\ \text { EPA } & \text { Environmental Protection Agency } \\ \text { FAA } & \text { Federal Aviation Administration } \\ \text { FHWA } & \text { Federal Highway Administration } \\ \text { FMCSR } & \text { Federal Motor Carrier Safety Regulations } \\ \text { FRA } & \text { Federal Railroad Administration } \\ \text { HM or HAZMAT } & \text { Hazardous Materials } \\ \text { HMR } & \text { Hazardous Materials Regulations } \\ \text { IATA } & \text { International Air Transport Association } \\ \text { ICAO } & \text { International Civil Aviation Organization } \\ \text { ICC } & \text { Interstate Commerce Commission } \\ \text { IMDG } & \text { International Maritime Dangerous Goods } \\ \text { IMO } & \text { International Maritime Organization } \\ \text { LNG } & \text { Liquified natural gas } \\ \text { NRC } & \text { Nuclear Regulatory Commission } \\ \text { OSHA } & \text { Occupational Safety and Health Administration } \\ \text { PCB } & \text { Polychlorinated biphenyl } \\ \text { RSPA } & \text { Research and Special Programs Administration } \\ \text { RFG } & \text { Regulatory Facility Guide } \\ \text { TCA } & \text { Tennessee Codes Annotated } \\ \text { TN } & \text { Tennessee } \\ \text { TPSC } & \text { Tennessee Public Service Commission }\end{array}$




\begin{tabular}{|c|c|c|c|c|c|c|c|c|c|c|c|c|c|c|}
\hline \multicolumn{15}{|c|}{ SHIPPER ACTIVITIES } \\
\hline \multirow{2}{*}{\multicolumn{2}{|c|}{$\begin{array}{l}\text { Stipper } \\
\text { Activity }\end{array}$}} & RSPA & FRA & FHWA & $I 0 C$ & EPA & OSHA & PAA & NRC & \multirow{2}{*}{$\begin{array}{c}\text { DOC } \\
15 \text { CFR } \\
19 \text { CFR }\end{array}$} & \multirow[b]{2}{*}{$\begin{array}{l}\text { Stale of } \\
\text { TN }\end{array}$} & \multirow[b]{2}{*}{$\begin{array}{c}\text { DOE } \\
\text { Onders }\end{array}$} & \multirow[b]{2}{*}{ MTA } & \multirow[b]{2}{*}{$\begin{array}{l}\text { IMO } \\
\text { MDO }\end{array}$} \\
\hline & & $\begin{array}{l}49 \text { CFR } \\
100-199\end{array}$ & $\begin{array}{l}49 \text { CFR } \\
200-299\end{array}$ & $\begin{array}{l}49 \mathrm{CFR} \\
300-349\end{array}$ & $\begin{array}{l}49 \text { CFR } \\
1000-1399\end{array}$ & $\begin{array}{l}40 \text { CFR } \\
1-799\end{array}$ & $29 \mathrm{CFR}$ & $14 \mathrm{CFK}$ & $10 \mathrm{CFR}$ & & & & & \\
\hline 1. & $\begin{array}{l}\text { Carrier } \\
\text { Qualifications }\end{array}$ & $\begin{array}{l}171.1 \\
171.2(\text { a) } \\
171.2(\text { b) } \\
174.7 \\
175.20 \\
176.13 \\
177.800\end{array}$ & $\begin{array}{l}213 \\
215 \\
219\end{array}$ & $\begin{array}{l}385 \\
387 \\
390.3\end{array}$ & $\begin{array}{l}1035 \\
1043.2 \\
1051 \\
1150-1174 \\
1312 \\
1330\end{array}$ & 263.11 & 1910.120 & 121 & 71.5 & & \begin{tabular}{l} 
TCA Title 65 \\
Chapper 15 \\
$655-15-107$ \\
$65-15-108$ \\
$65-15-110$ \\
$65-15-113$ \\
\hdashline TrSC Rule \\
Numbers: \\
$1220-2-1.18$ \\
$1220-2-1.19$ \\
$1220-2-1 \cdot-20$ \\
$1220-2-1-32$
\end{tabular} & $\begin{array}{l}1540.1 \\
5480.4\end{array}$ & 1.4 & \\
\hline 2 & $\begin{array}{l}\text { Carrier } \\
\text { Selection }\end{array}$ & $\begin{array}{l}171.1 \\
171.2(a) \\
171.2(\mathrm{~b}) \\
174.7 \\
175.20 \\
176.13 \\
177.800\end{array}$ & $\begin{array}{l}213 \\
215 \\
219\end{array}$ & $\begin{array}{l}385 \\
387 \\
390.3\end{array}$ & $\begin{array}{l}1035 \\
1043.2 \\
1051 \\
1150-1174 \\
1312 \\
1330\end{array}$ & 263.11 & 1910.120 & 121 & 71.5 & & $\begin{array}{l}\text { TCA } \\
65-15-107 \\
65-15-108 \\
65-15-110 \\
65-15-113 \\
\text { TPSC Rule } \\
\text { Numbers: } \\
1220-2-1 \cdot 18 \\
1220-2-1-19 \\
1220-2-1-20 \\
1220-2-1-.32\end{array}$ & $\begin{array}{l}540.1 \\
5480.4\end{array}$ & & \\
\hline 3. & $\begin{array}{l}\text { Emergency } \\
\text { Response }\end{array}$ & 172 Subpan G & 225 & 390.15 & & $\begin{array}{l}117 \\
302\end{array}$ & 1910.120 & & 71.5 & & $\begin{array}{l}\text { TCA } \\
58-2-301 \\
\text { et seq } \\
\\
49 \text { CPR } \\
172 \text { Subpan G } \\
225 \\
390.15 \\
\\
40 \text { CrR } \\
117 \\
302 \\
\end{array}$ & $5000.3 \mathrm{~B}$ & $\begin{array}{l}\text { 29.2I } \\
\text { USG-17, } \\
8.1 .6 .12\end{array}$ & \\
\hline & Hazardous Material & $\begin{array}{l}171.8 \\
172\end{array}$ & & & & & & & & & $\begin{array}{l}99 \mathrm{CPR} \\
171.8\end{array}$ & $\begin{array}{l}1540.1 \\
5480.3\end{array}$ & $\begin{array}{l}3 \\
49 \mathrm{CFR} \\
171.11\end{array}$ & $\begin{array}{l}49 \mathrm{CPR} \\
171.12\end{array}$ \\
\hline & $\begin{array}{l}\text { Hazardous } \\
\text { Substance }\end{array}$ & $\begin{array}{l}171.8 \\
172101, \text { App. A }\end{array}$ & & & & $\begin{array}{l}117 \\
302\end{array}$ & & & & & $\begin{array}{l}19 \mathrm{CFR} \\
171.8 \\
10 \mathrm{CFR} \\
117 \\
302\end{array}$ & $\begin{array}{l}1540.1 \\
5480.3\end{array}$ & $\begin{array}{l}29.21 \\
\text { USG }-01 \\
3.9\end{array}$ & $\begin{array}{l}\text { ALL } \\
\text { CODES } \\
\text { APPLY }\end{array}$ \\
\hline
\end{tabular}




\begin{tabular}{|c|c|c|c|c|c|c|c|c|c|c|c|c|c|}
\hline \multicolumn{14}{|c|}{ SHIPPER ACTIVITIES } \\
\hline Stipper & RSPA & ITRA & FITA & $\mathrm{IOC}$ & EPA & OSIIA & PAA & NRC & DOC & & & & \\
\hline Activity & $\begin{array}{l}49 \text { CFR } \\
100-199\end{array}$ & $\begin{array}{l}49 \text { CFR } \\
200-299\end{array}$ & $\begin{array}{l}99 \text { CFR } \\
300-399\end{array}$ & $\begin{array}{c}49 \text { CFR } \\
1000.1399\end{array}$ & $\begin{array}{l}40 \text { CFR } \\
1.799\end{array}$ & $29 \mathrm{ClR}$ & $14 \mathrm{CFR}$ & $10 \mathrm{CFR}$ & $\begin{array}{l}15 \text { CFR } \\
19 \text { CFR }\end{array}$ & $\begin{array}{l}\text { Stale of } \\
\text { TN }\end{array}$ & $\begin{array}{l}\text { DOE } \\
\text { Onders }\end{array}$ & LATA & $\begin{array}{l}\text { IMOI } \\
\text { IMDG }\end{array}$ \\
\hline 6. Hazardous Waste & $\begin{array}{l}171.3 \\
171.8\end{array}$ & & & & 261.3 & & & & & $\begin{array}{l}49 \mathrm{CPR} \\
171.8 \\
\\
40 \mathrm{CPR} \\
261.3\end{array}$ & $\begin{array}{l}1540.1 \\
5480.3\end{array}$ & $\begin{array}{l}29.21 \\
\text { USG. } 01\end{array}$ & $\begin{array}{l}\text { ALL } \\
\text { CODES } \\
\text { APPLY }\end{array}$ \\
\hline $\begin{array}{ll}\text { 7. } & \text { HM } \\
\text { Segregation }\end{array}$ & $\begin{array}{l}174.81 \\
175.78 \\
176 \text { Subpan D } \\
177.842 \\
177.848\end{array}$ & & & & & & & 71.5 & & $\begin{array}{l}\text { 19 CPR } \\
174.81 \\
175.78 \\
176.83 \\
177.842 \\
177.848 \\
\end{array}$ & $\begin{array}{l}1540.1 \\
5480.3\end{array}$ & $\begin{array}{l}9.3 .2 \\
\text { Table } \\
9.3 . \mathrm{A} \\
\\
-\end{array}$ & $\begin{array}{l}\text { Gen. } \\
\text { Intro. } \\
\text { Sect.15,18 }\end{array}$ \\
\hline 8. Labeling & 172 Subpart E & & & & 262.31 & & & 71.5 & & $\begin{array}{l}49 \mathrm{CPR} \\
172 \text { Subpant E } \\
40 \mathrm{CFR} \\
26231\end{array}$ & $\begin{array}{l}1540.1 \\
5480.3\end{array}$ & $\begin{array}{l}6.4, \\
7.2\end{array}$ & $\begin{array}{l}\text { Gen. } \\
\text { Intro. } \\
\text { Sect } 7,8\end{array}$ \\
\hline $\begin{array}{l}\text { 9. Loading } \\
\text { Unloading }\end{array}$ & $\begin{array}{l}174 \text { Subpart C } \\
175 \text { Subpart B } \\
176 \text { Subpart C } \\
177 \text { Subpart B }\end{array}$ & & 392.9 & & & & & 71.5 & & $\begin{array}{l}\text { 19 CPR } \\
174 \text { Subpan C } \\
175 \text { Subpart B } \\
176 \text { Subpart C } \\
177 \text { Subpart B }\end{array}$ & $\begin{array}{l}540.1 \\
5480.3\end{array}$ & 9.3 & $\begin{array}{l}\text { Gen. } \\
\text { Intro. } \\
\text { Sect } 17\end{array}$ \\
\hline 10. Manifest & 172.205 & & & & $\begin{array}{l}262 \text { Subpart B } \\
263 \text { Subpart B }\end{array}$ & & & & & $\begin{array}{l}9 \text { CPR } \\
172205 \\
\text { 40 CPR } \\
262 \text { Subpart B } \\
263 \text { Subpart B }\end{array}$ & $\begin{array}{l}1540.1 \\
5480.1 \mathrm{~B} \\
5480.3\end{array}$ & $\begin{array}{l}29.21 \\
\text { USG-01 } \\
\text { II(a) }\end{array}$ & $\begin{array}{l}\text { Gen. } \\
\text { Intro. } \\
\text { Sect.9 }\end{array}$ \\
\hline 11. Marking & 172 Subpan D & & & & 262.32 & & & 71.5 & & $\begin{array}{l}49 \text { CFR } \\
172 \text { Subpan D } \\
40 \mathrm{Cl} / \mathrm{R} \\
26232\end{array}$ & $\begin{array}{l}1540.1 \\
5480.3\end{array}$ & $\begin{array}{l}6.3 \\
7.1\end{array}$ & $\begin{array}{l}\text { Gen. } \\
\text { Intro. } \\
\text { Sect7.7.8 } \\
\text { Annex I }\end{array}$ \\
\hline 12 Packaging & $\begin{array}{l}173 \\
178 \\
179 \\
180\end{array}$ & & & & 26230 & & & 71 & & $\begin{array}{l}99 \mathrm{CPR} \\
173 \\
178 \\
179 \\
180 \\
40 \mathrm{CrR} \\
26230\end{array}$ & $\begin{array}{l}1540.1 \\
1540.2 \\
5480.3\end{array}$ & $\overline{5,10}$ & Annex I \\
\hline $\begin{array}{l}\text { 13. PCB/ } \\
\text { Asbestos }\end{array}$ & 173.140 & & & & $\begin{array}{l}761 \\
763\end{array}$ & & & & & $\begin{array}{l}49 \mathrm{CPR} \\
173.140 \\
40 \mathrm{CPR} \\
761 \\
763\end{array}$ & $\begin{array}{l}1540.1 \\
5480.3\end{array}$ & $\begin{array}{l}3.9 \\
4.2\end{array}$ & $\begin{array}{l}\text { Vol.V } \\
\text { Class } 9\end{array}$ \\
\hline
\end{tabular}




\begin{tabular}{|c|c|c|c|c|c|c|c|c|c|c|c|c|c|}
\hline \multicolumn{14}{|c|}{ SHIPPER ACTIVITIES } \\
\hline \multirow{2}{*}{$\begin{array}{l}\text { Sthipper } \\
\text { Activity }\end{array}$} & RSPA & FRA & FIIWA & $\overline{10 C}$ & $\overline{\text { EPA }}$ & $\overline{\text { osila }}$ & $\overline{\text { PAA }}$ & NRC & \multirow{2}{*}{$\begin{array}{c}\text { DOC } \\
15 \text { CFR } \\
19 \text { CFR }\end{array}$} & \multirow[b]{2}{*}{$\begin{array}{c}\text { Sale of } \\
\text { TN }\end{array}$} & \multirow[b]{2}{*}{$\begin{array}{l}\text { IDOE } \\
\text { Onders }\end{array}$} & \multirow[b]{2}{*}{ LATA } & \multirow[b]{2}{*}{$\begin{array}{l}\text { IMOM } \\
\text { IMDO }\end{array}$} \\
\hline & $\begin{array}{l}49 \text { CFR } \\
100-199\end{array}$ & $\begin{array}{l}19 \mathrm{CFR} \\
200.299\end{array}$ & $\begin{array}{l}9 \text { CFR } \\
300-399\end{array}$ & $\begin{array}{l}49 \text { CFR } \\
1000-1399\end{array}$ & $\begin{array}{l}0 \text { CFR } \\
1.799\end{array}$ & $29 \mathrm{CHR}$ & $14 \mathrm{CFR}$ & $10 \mathrm{CFK}$ & & & & & \\
\hline 14. Placarding & 172 Subpart F & & & & 26233 & & & 71.5 & & $\begin{array}{l}49 \mathrm{CPR} \\
172 \text { Subpart F } \\
40 \mathrm{CFR} \\
26233\end{array}$ & $\begin{array}{l}1540.1 \\
5480.3\end{array}$ & & $\begin{array}{l}\text { Gen. } \\
\text { Intro. } \\
\text { Sect } 7.8\end{array}$ \\
\hline $\begin{array}{l}\text { 15. Radioactive } \\
\text { Material }\end{array}$ & 173 Subpart I & & & & $\begin{array}{l}117 \\
302\end{array}$ & & & $\begin{array}{l}20.3(13), \\
70\end{array}$ & & $\begin{array}{l}9 \text { CPR } \\
173 \text { Subpart I } \\
40 \text { CFR } \\
117 \\
302\end{array}$ & $\begin{array}{l}1540.1 \\
5480.1 \mathrm{~B}\end{array}$ & $\begin{array}{l}1.3 .3 .3 \\
6\end{array}$ & $\begin{array}{l}\text { Vol.1v, } \\
\text { Class } 7\end{array}$ \\
\hline 16. Reconds & 172 Subpart C & & & $\begin{array}{l}1035 \\
1051 \\
1081\end{array}$ & 262 Subpart $D$ & 1910.120 & & 71.5 & & $\begin{array}{l}9 \text { CFR } \\
172 \text { Subpan C } \\
1035 \\
1051 \\
1081 \\
\\
40 \text { CFR } \\
262 \text { Subpart D }\end{array}$ & $\begin{array}{l}1540.1 \\
5480.1 \mathrm{~B} \\
5480.3\end{array}$ & $\begin{array}{l}29.21 \\
\text { USG-18 }\end{array}$ & $\begin{array}{l}\text { Gen. } \\
\text { Intro. } \\
\text { Sect9 }\end{array}$ \\
\hline 17. Reporting & $\begin{array}{l}171.15 \\
171.16\end{array}$ & 225 & 390.15 & & $\begin{array}{l}117 \\
263.30 \\
302\end{array}$ & 1910.120 & & 71.5 & & $\begin{array}{l}9 \text { CFR } \\
171.15 \\
171.16 \\
390.15\end{array}$ & $\begin{array}{l}1540.1 \\
5000.3 \mathrm{~B} \\
5480.3\end{array}$ & 9.5 .5 & \\
\hline $\begin{array}{l}\text { 18. Shipping } \\
\text { Papers }\end{array}$ & 172 Subpant C & & & $\begin{array}{l}1035 \\
1051 \\
1081\end{array}$ & $\begin{array}{l}262 \text { Subpart B } \\
263 \text { Subpar B }\end{array}$ & & & 71.5 & & $\begin{array}{l}19 \text { CPR } \\
172 \text { Subpant C } \\
40 \text { CPR } \\
262 \text { Subpan B } \\
263 \text { Subpart B }\end{array}$ & $\begin{array}{l}1540.1 \\
5480.3\end{array}$ & $\begin{array}{l}29.21 \\
\text { USG-17 } \\
6.6 \\
8\end{array}$ & $\begin{array}{l}\text { Gen. } \\
\text { Intro. } \\
\text { SecL.7.9 }\end{array}$ \\
\hline 19. Temporary Storage & $\begin{array}{l}174.81 \\
175.78 \\
176.83 \\
177.842 \\
177.848\end{array}$ & & & & $\begin{array}{l}26234 \\
263.12\end{array}$ & & & 71.5 & & $\begin{array}{l}9 \mathrm{CPR} \\
174.81 \\
175.78 \\
17.83 \\
177.842 \\
177.848 \\
40 \mathrm{CPR} \\
262.34 \\
263.12\end{array}$ & $\begin{array}{l}1540.1 \\
5480.3\end{array}$ & 9.2 & \\
\hline
\end{tabular}




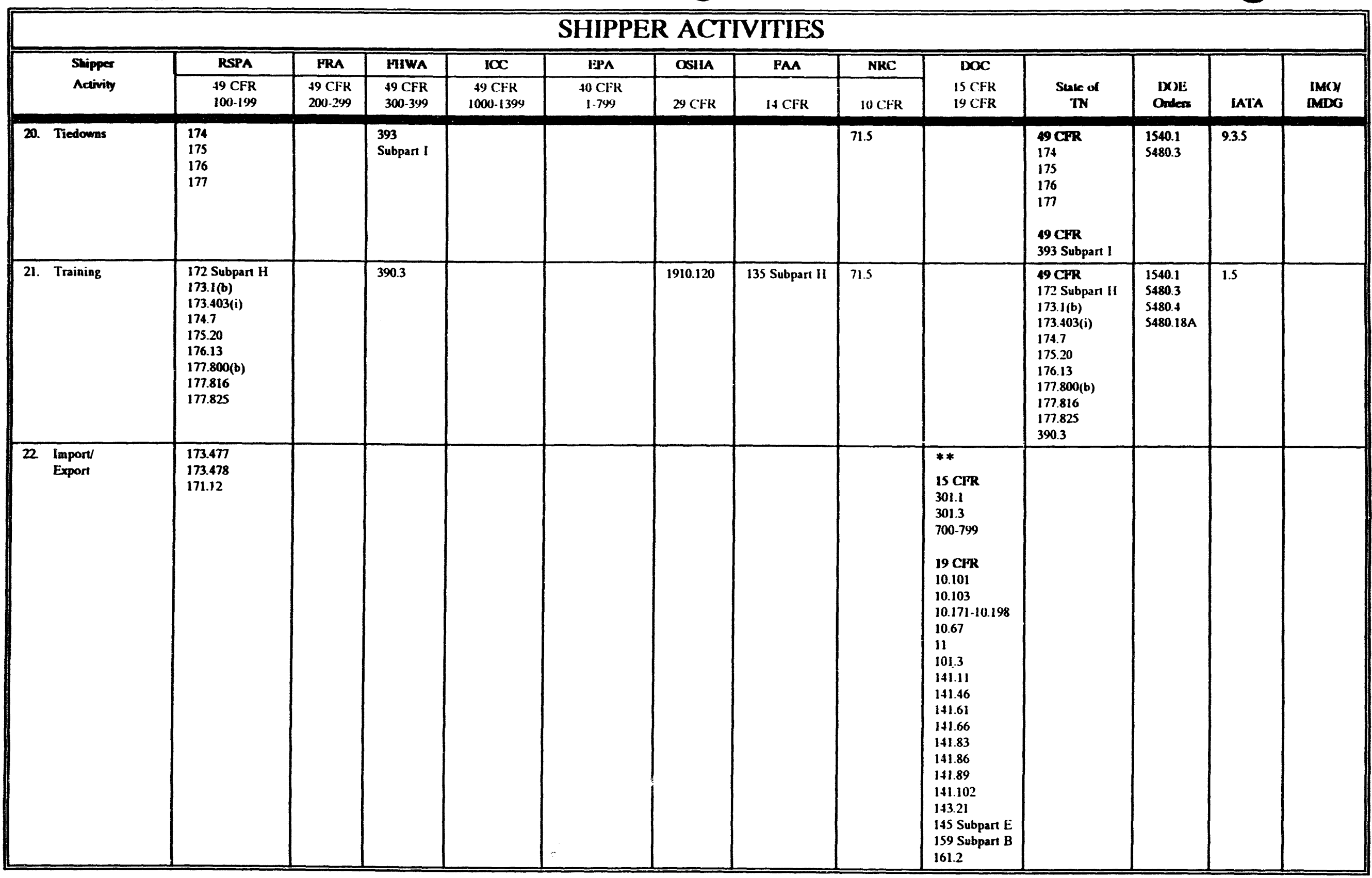

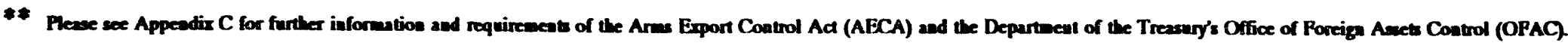

RFG for TIENNESSEE: Page $1-4$ 


\begin{tabular}{|c|c|c|c|c|c|c|c|c|c|c|c|c|}
\hline \multicolumn{13}{|c|}{ CARRIER ACTIVITIES } \\
\hline Carriar & RSPA & PRA & FHWA & IOC & EPA & CSHA & PAA & NRC & \multirow[b]{2}{*}{$\begin{array}{l}\text { Suak of } \\
\text { TN }\end{array}$} & \multirow[b]{2}{*}{$\begin{array}{l}\text { DOE } \\
\text { Onders }\end{array}$} & \multirow[b]{2}{*}{ IATA } & \multirow[b]{2}{*}{$\begin{array}{l}\text { MMOM } \\
\text { MDG }\end{array}$} \\
\hline Activity & $\begin{array}{l}49 \text { CFR } \\
100.199\end{array}$ & $\begin{array}{l}49 \mathrm{CFR} \\
200-299\end{array}$ & $\begin{array}{l}49 \text { CFR } \\
300-399\end{array}$ & $\begin{array}{c}49 \text { CFR } \\
1000.1399\end{array}$ & $\begin{array}{c}40 \text { CFR } \\
1.799\end{array}$ & 29 CFR & $14 \mathrm{CFR}$ & $10 \mathrm{CFR}$ & & & & \\
\hline $\begin{array}{l}\text { 1. Blocking/ } \\
\text { Bracing }\end{array}$ & 177 Subpart B & & 393.104 & & 26231 & & & 71.5 & $\begin{array}{l}49 \text { CFR } \\
177 \text { Subpart B } \\
40 \mathrm{CHR} \\
262.31\end{array}$ & $\begin{array}{l}1540.1 \\
5480.3 \\
5480.4\end{array}$ & 9.3 & $\begin{array}{l}\text { Gen. } \\
\text { Intro. } \\
\text { Sect.12 } \\
\text { App.Resol. } \\
\text { A.288(VIII) }\end{array}$ \\
\hline 2 Drug Testing & & 219 & 391 Subpan H & & & & 121 & & $\begin{array}{l}49 \text { CFR } \\
219 \\
391 \text { Subpart II }\end{array}$ & $\begin{array}{l}1540.1 \\
5480.4\end{array}$ & & \\
\hline $\begin{array}{l}\text { 3. Emergency } \\
\text { Response }\end{array}$ & 172 Subpan $\mathrm{G}$ & 225 & 390.15 & & $\begin{array}{l}263.30 \\
263.31\end{array}$ & 1910.120 & & & $\begin{array}{l}\text { TCA } \\
\text { 58-2-301 el seq } \\
49 \text { CFR } \\
172 \text { Subpart G } \\
225 \\
390.15 \\
40 \text { CFR } \\
263.30 \\
263.31 \\
\end{array}$ & 5000.38 & $\begin{array}{l}29.21 \\
\text { USG-17 }\end{array}$ & \\
\hline $\begin{array}{l}\text { 4. Hazardous } \\
\text { Malerial } \\
\end{array}$ & $\begin{array}{l}171.8 \\
172 \\
\end{array}$ & & & & & & & 71.5 & $\begin{array}{l}9 \mathrm{CFR} \\
171.8 \\
\end{array}$ & $\begin{array}{l}1540.1 \\
5480.3 \\
\end{array}$ & 3 & $\begin{array}{l}\text { ALL CODES } \\
\text { APPLY }\end{array}$ \\
\hline 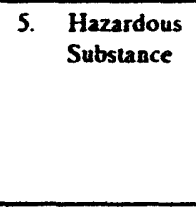 & 171.8 & & & & $\begin{array}{l}117 \\
302\end{array}$ & & & & $\begin{array}{l}99 \mathrm{CPK} \\
171.8 \\
40 \mathrm{CFR} \\
117 \\
302\end{array}$ & $\begin{array}{l}540.1 \\
5480.3\end{array}$ & $\begin{array}{l}29.21 \\
\text { USG-01 } \\
3.9\end{array}$ & $\begin{array}{l}\text { ALL CODES } \\
\text { APPLY }\end{array}$ \\
\hline 6. $\begin{array}{l}\text { Hazardous } \\
\text { Waste }\end{array}$ & $\begin{array}{l}171.3 \\
171.8\end{array}$ & & & & $\begin{array}{l}260 \\
261.3\end{array}$ & & & & $\begin{array}{l}9 \mathrm{CPR}^{2} \\
171.8 \\
40 \mathrm{Cl} \\
260 \\
261.3\end{array}$ & $\begin{array}{l}540.1 \\
5480.3\end{array}$ & $\begin{array}{l}2.9 .2 \\
\text { USG-01 }\end{array}$ & $\begin{array}{l}\text { ALL CODES } \\
\text { APPLY }\end{array}$ \\
\hline 7. $\begin{array}{l}\text { HM } \\
\text { Segregation }\end{array}$ & $\begin{array}{l}174.81 \\
175.78 \\
176 \text { Subpan D } \\
177.842 \\
177.848\end{array}$ & & & & & & & 71.5 & $\begin{array}{l}99 \text { CPR } \\
174.8 \mathrm{i} \\
175.78 \\
176.83 \\
177.842 \\
177.848\end{array}$ & $\begin{array}{l}1540.1 \\
5480.3\end{array}$ & $\begin{array}{l}9.3 .2 \\
\text { Table } \\
9.3 . \mathrm{A}\end{array}$ & $\begin{array}{l}\text { Gen. } \\
\text { Intro. } \\
\text { Sect.15,17 }\end{array}$ \\
\hline 8. Labeling & 172 Subpart E & & & & 26231 & & & 71.5 & $\begin{array}{l}9 \text { CrR } \\
172 \text { Subpart E } \\
40 \text { CFR } \\
26231\end{array}$ & $\begin{array}{l}540.1 \\
5480.3\end{array}$ & $\begin{array}{l}6.4 \\
7.2\end{array}$ & $\begin{array}{l}\text { Gen. } \\
\text { Intro. } \\
\text { Sect } 7.8\end{array}$ \\
\hline
\end{tabular}

RFG for TI:NNI:SSIII, Page 1-5 


\begin{tabular}{|c|c|c|c|c|c|c|c|c|c|c|c|c|}
\hline \multicolumn{13}{|c|}{ CARRIER ACTIVITIES } \\
\hline Camier & RSPA & FRA & FHWA & 100 & EPA & OSHA & PAA & NRC & \multirow[b]{2}{*}{$\begin{array}{l}\text { Stave of } \\
\text { TN }\end{array}$} & \multirow[b]{2}{*}{$\begin{array}{l}\text { DXSE } \\
\text { Onders }\end{array}$} & \multirow[b]{2}{*}{ IATA } & \multirow[b]{2}{*}{$\begin{array}{l}\text { IMCD } \\
\text { IMDG }\end{array}$} \\
\hline Activity & $\begin{array}{l}99 \mathrm{CFR} \\
100-199\end{array}$ & $\begin{array}{l}49 \text { CFR } \\
200-299\end{array}$ & $\begin{array}{l}49 \mathrm{CFR} \\
300-399\end{array}$ & $\begin{array}{c}49 \mathrm{CFR} \\
1000-1399\end{array}$ & $\begin{array}{c}40 \text { CFK } \\
1.799\end{array}$ & $29 \mathrm{CFR}$ & IH CTR & $10 \mathrm{CFR}$ & & & & \\
\hline 9. Licensing & 177.804 & & 383 & & & & & 71.5 & $\begin{array}{l}19 \text { CFR } \\
177.804 . \\
383\end{array}$ & $\begin{array}{l}1540.1 \\
5480.4\end{array}$ & & \\
\hline $\begin{array}{l}\text { 10. Loading/ } \\
\text { Unloading }\end{array}$ & $\begin{array}{l}174 \text { Subpart C } \\
175 \text { Subpart B } \\
176 \text { Subpart C } \\
177 \text { Subpart B }\end{array}$ & & 3929 & & & & & 71.5 & $\begin{array}{l}\text { 99 CPR } \\
174 \text { Subpart C } \\
175 \text { Subpart B } \\
176 \text { Subpart C } \\
177 \text { Subpart B }\end{array}$ & $\begin{array}{l}1540.1 \\
5480.3\end{array}$ & 9.3 & $\begin{array}{l}\text { Gen. } \\
\text { Intro. } \\
\text { Sect.17 }\end{array}$ \\
\hline 11. Marking & 172 Subpart D & & & & 262.32 & & & 71.5 & $\begin{array}{l}49 \text { CFR } \\
172 \text { Subpart D } \\
40 \text { CrR } \\
26232\end{array}$ & $\begin{array}{l}1540.1 \\
5480.3\end{array}$ & $\begin{array}{l}6.3 \\
7.1\end{array}$ & $\begin{array}{l}\text { Gen. } \\
\text { Intro. } \\
\text { SecL7.8 } \\
\text { Annex I }\end{array}$ \\
\hline $12 \begin{array}{c}\text { Non-Haz } \\
\text { Material }\end{array}$ & & & & & & & & & & $\begin{array}{l}1540.1 \\
5480.4\end{array}$ & & \\
\hline $\begin{array}{l}\text { 13. Operator } \\
\text { Quality }\end{array}$ & & & 391 & & & & & & & $\begin{array}{l}1540.1 \\
1540.2 \\
5480.3 \\
5480.4\end{array}$ & 1.4 & \\
\hline 14. Packaging & $\begin{array}{l}173 \\
178 \\
179 \\
180\end{array}$ & & & & 26230 & & & 71 & $\begin{array}{l}49 \mathrm{CPR} \\
173 \\
178 \\
179 \\
180 \\
\\
40 \mathrm{CPR} \\
26230\end{array}$ & $\begin{array}{l}1540.1 \\
5480.3\end{array}$ & 5,10 & $\begin{array}{l}\text { Gen. } \\
\text { Intro. } \\
\text { Sect.10,17 } \\
\text { Aanex I }\end{array}$ \\
\hline 15. Placarding & 172 Subpart F & & & & 26233 & & & $\begin{array}{l}20.3 \\
\text { (13) }\end{array}$ & $\begin{array}{l}19 \text { CrR } \\
172 \text { Subpart F } \\
40 \text { ClK } \\
26233\end{array}$ & $\begin{array}{l}1540.1 \\
5480.1 \\
5480.3\end{array}$ & & $\begin{array}{l}\text { Gen. } \\
\text { Intro } \\
\text { Sect } 7.8\end{array}$ \\
\hline 16. Records & $\begin{array}{l}\text { 171.16(b) } \\
172 \text { Subpant C }\end{array}$ & & $\begin{array}{l}397.19 \\
390.15\end{array}$ & $\begin{array}{l}1035 \\
1051 \\
1081\end{array}$ & 262 Subpan D & & & 71.5 & $\begin{array}{l}9 \text { CrR } \\
171.16(b) \\
390.15 \\
397.19 \\
172 \text { Subpart C } \\
1035 \\
1051 \\
1081 \\
\\
40 \text { CrR } \\
262 \text { Subpart D }\end{array}$ & $\begin{array}{l}1540.1 \\
5480.1 \mathrm{~B} \\
5480.3\end{array}$ & $\begin{array}{l}2.9 .2 \\
\text { USG-18 }\end{array}$ & $\begin{array}{l}\text { Gen. } \\
\text { Intro. } \\
\text { Sect.9 }\end{array}$ \\
\hline
\end{tabular}




\begin{tabular}{|c|c|c|c|c|c|c|c|c|c|c|c|c|}
\hline \multirow{2}{*}{$\begin{array}{l}\text { Cantier } \\
\text { Activity }\end{array}$} & RSPA & $\overline{\text { FRA }}$ & FHWA & $10 x$ & EPA & Osin & $\overline{\text { PAA }}$ & NRC & \multirow[b]{2}{*}{$\begin{array}{l}\text { Stave of } \\
\text { TN }\end{array}$} & \multirow[b]{2}{*}{$\begin{array}{l}\text { DOE } \\
\text { Ondere }\end{array}$} & \multirow[b]{2}{*}{ IATA } & \multirow[b]{2}{*}{$\begin{array}{l}\text { IMON } \\
\text { IMDC }\end{array}$} \\
\hline & $\begin{array}{l}49 \text { CFR } \\
100-199\end{array}$ & $\begin{array}{l}9 \text { CFR } \\
200-209\end{array}$ & $\begin{array}{l}49 \text { CFR } \\
300-3 \times 9\end{array}$ & $\begin{array}{c}49 \text { CFK } \\
1000-1399\end{array}$ & $\begin{array}{l}+0 \text { CFR } \\
1.799\end{array}$ & 29 CHR & $14(\% \mathrm{~K}$ & $10 \mathrm{CFR}$ & & & & \\
\hline 17. Reporting & $\begin{array}{l}171.15 \\
171.16\end{array}$ & 225 & 390.15 & & $\begin{array}{l}117 \\
263.30 \\
302\end{array}$ & 1910.120 & & 71.5 & $\begin{array}{l}\text { TCA } \\
58-2-301 \text { et seq } \\
\\
49 \mathrm{CFR} \\
171.15 \\
171.16 \\
225 \\
390.15 \\
\\
40 \mathrm{CFR} \\
117 \\
263.30 \\
302\end{array}$ & $\begin{array}{l}1540.1 \\
5000.3 B \\
5480.3\end{array}$ & 9.5 .5 & \\
\hline 18. Shipping Papers & 172 Subpart C & & & $\begin{array}{l}1035 \\
1051 \\
.081\end{array}$ & $\begin{array}{l}262 \text { Subpan B } \\
263 \text { Subpart B }\end{array}$ & & & 71.5 & $\begin{array}{l}\text { 49 CFR } \\
172 \text { Subpart C } \\
40 \text { CrR } \\
262 \text { Subpart B } \\
263 \text { Subpart B }\end{array}$ & $\begin{array}{l}1540.1 \\
5480.3\end{array}$ & $\begin{array}{l}29.21 \\
\text { USG-17 } \\
6.6 \\
8\end{array}$ & $\begin{array}{l}\text { Gen. } \\
\text { Intro. } \\
\text { Sect } 7,9\end{array}$ \\
\hline 19. Tiedowns & $\begin{array}{l}174 \\
175 \\
176 \\
177\end{array}$ & & 393 Subpant I & & & & & 71.5 & $\begin{array}{l}9 \text { CPR } \\
174 \\
175 \\
176 \\
177 \\
393 \text { Subpart I }\end{array}$ & $\begin{array}{l}1540.1 \\
5480.3 \\
5480.4\end{array}$ & 9.3 .5 & $\begin{array}{l}\text { Gen. } \\
\text { Intro. } \\
\text { Sect 12 } \\
\text { Resol. } \\
\text { A.288(VIII) }\end{array}$ \\
\hline 20. Training & $\begin{array}{l}172 \text { Subparn H } \\
174.7 \\
175.20 \\
176.13 \\
177.800(b) \\
177.816 \\
177.825\end{array}$ & & 390.3 & & & 1910.120 & 135 Subpart H & 71.5 & $\begin{array}{l}9 \text { CFR } \\
172 \text { Subpan H } \\
174.7 \\
175.20 \\
176.13 \\
177.800 \text { (b) } \\
177.816 \\
177.825 \\
390.3\end{array}$ & $\begin{array}{l}1540.1 \\
5480.3 \\
5480.4\end{array}$ & 1.5 & \\
\hline $\begin{array}{l}\text { 21. } \begin{array}{l}\text { Vehicle } \\
\text { laspection }\end{array}\end{array}$ & 177.804 & & $\begin{array}{l}3927 \\
3928 \\
3929 \\
396\end{array}$ & & & & $\begin{array}{l}109 \text { Sub- } \\
\text { chapter G, } \\
121\end{array}$ & & $\begin{array}{l}9 \mathrm{Cl} \mathrm{K} \\
177.804 \\
3927 \\
3928 \\
3929 \\
396\end{array}$ & $\begin{array}{l}1540.1 \\
5480.4\end{array}$ & & \\
\hline 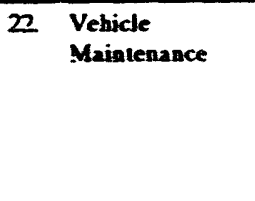 & 377.804 & & $\begin{array}{l}3927 \\
392.8 \\
392.9 \\
396\end{array}$ & & & & $\begin{array}{l}109 \text { Sub- } \\
\text { chapter (i, } \\
121\end{array}$ & 71.5 & $\begin{array}{l}9 \mathrm{ClR} \\
177.804 \\
392.7 \\
3928 \\
3929 \\
396\end{array}$ & $\begin{array}{l}1540.1 \\
5480.4\end{array}$ & & \\
\hline
\end{tabular}




\begin{tabular}{|c|c|c|c|c|c|c|c|c|c|c|c|c|}
\hline \multicolumn{13}{|c|}{ RECEIVER ACTIVITIES } \\
\hline & RSPA & Fin & FIWA & $10 x$ & EPA & $\overline{\text { OSIIA }}$ & $\overline{P M A}$ & $\overline{\text { NRC }}$ & DOC & & & \\
\hline $\begin{array}{l}\text { Roociver } \\
\text { Activity }\end{array}$ & $\begin{array}{l}49 \text { CFR } \\
100-199\end{array}$ & $\begin{array}{l}99 \text { CFR } \\
200-299\end{array}$ & $\begin{array}{l}49 \text { CFR } \\
300-359\end{array}$ & $\begin{array}{c}49 \mathrm{CFR} \\
10001-1399\end{array}$ & $\begin{array}{c}\text { S0 CFR } \\
1.799\end{array}$ & $29 \mathrm{CHK}$ & $14 \mathrm{CHK}$ & $10 \mathrm{CHR}$ & $\begin{array}{l}15 \text { CFR } \\
19 \text { CFR }\end{array}$ & $\begin{array}{l}\text { Sulce of } \\
\text { TN }\end{array}$ & $\begin{array}{l}\text { DOE } \\
\text { Ordere }\end{array}$ & LATA \\
\hline $\begin{array}{l}\text { 1. Emergency } \\
\text { Response }\end{array}$ & & & & & $\begin{array}{l}117 \\
264 \text { Subpant C } \\
302\end{array}$ & $\begin{array}{l}1910.38 \\
1910.120\end{array}$ & & & & $\begin{array}{l}\text { TCA } \\
\text { 58-2-201 } \\
\text { et seq }\end{array}$ & $5000.3 B$ & $\begin{array}{l}29.21 \\
\text { USG-17 }\end{array}$ \\
\hline $2 \quad \begin{array}{l}\text { HM } \\
\text { Segregation }\end{array}$ & & & & & & & & & & & & $\begin{array}{l}9.3 .2 \\
\text { Table } \\
9.3 . \mathrm{A}\end{array}$ \\
\hline $\begin{array}{l}\text { 3. Ispection } \\
\text { of Reccipt }\end{array}$ & & & & & & & & & & & 1540.1 & \\
\hline 4. Records & & & & & 264 Subpant E & & & & & & $5000.3 \mathrm{~B}$ & $\begin{array}{l}29.24 \\
\text { USG-18 }\end{array}$ \\
\hline 5. Reporting & & & & & $\begin{array}{l}117 \\
302\end{array}$ & 1910.120 & & & & & $5000.3 \mathrm{~B}$ & 9.5 .5 \\
\hline 6. Training & & & & & $\begin{array}{l}264.16 \\
265.16\end{array}$ & 1910.120 & & & & $\begin{array}{l}0 \text { CPR } \\
264.16 \\
265.16 \\
29 \mathrm{CPR} \\
1910.120\end{array}$ & $\begin{array}{l}5480.18 \mathrm{~A} \\
5480.20\end{array}$ & \\
\hline 7. Imporrfexpon & $\begin{array}{l}171.12 \\
173.477 \\
173.478\end{array}$ & & & & & & & & $\begin{array}{l}\text { 15 CPR } \\
301.1 \\
301.3 \\
700.799 \\
19 \text { CrR } \\
10.67 \\
10.101 \\
10.103 \\
10.171-10.198 \\
11 \\
101.3 \\
141.11 \\
111.46 \\
141.61 \\
141.66 \\
141.83 \\
141.86 \\
141.89 \\
141.102 \\
143.21 \\
145 \text { Subpart E } \\
159 \text { Subpart B } \\
161.2\end{array}$ & & & \\
\hline
\end{tabular}

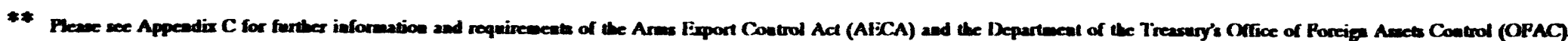





\begin{tabular}{|c|c|c|c|c|c|c|c|c|c|}
\hline \multicolumn{10}{|c|}{ 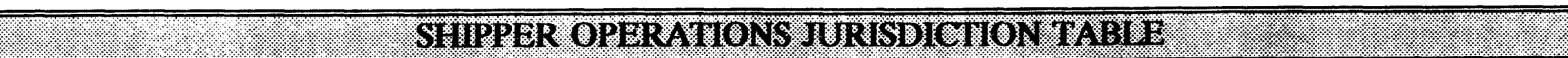 } \\
\hline \multirow[b]{2}{*}{$\begin{array}{l}\text { SHIPPER OPERATIONS } \\
\text { DESCRIPTION }\end{array}$} & \multicolumn{9}{|c|}{ Regulatory Agency/Applicable Regulations } \\
\hline & $\begin{array}{c}\text { RSPA } \\
\text { HMR } \\
49 \text { CFR 171.11, } \\
171.12\end{array}$ & $\begin{array}{l}\text { ICAO/ } \\
\text { IATA }\end{array}$ & $\begin{array}{c}\text { NRC } \\
10 \text { CFR } 71\end{array}$ & $\begin{array}{l}\text { DOE } \\
\text { Orders }\end{array}$ & $\begin{array}{c}\text { EPA } \\
40 \text { CFR 61, 117, } \\
240-272,302 \\
\text { (CERCLA) 760-761 }\end{array}$ & $\begin{array}{c}\text { OSHA } \\
29 \mathrm{CFR} \\
1900-1926\end{array}$ & $\begin{array}{c}\text { TPSC, TCA } \\
65-15-110 \\
65-15-113\end{array}$ & MOMMDG & $\begin{array}{l}\text { DOC }{ }^{* *} \\
15 \text { CFR } \\
19 \text { CFR }\end{array}$ \\
\hline \multicolumn{10}{|c|}{ INTERNATIONAL SHIPPING OPERATIONS } \\
\hline $\begin{array}{l}\text { Non Hazmat } \\
\text { Non Radioactive } \\
\text { Non Waste } \\
\text { Non Haz Substance }\end{array}$ & & & & Note 2 & & & * & & * \\
\hline $\begin{array}{ll}2 . & \text { Hazmat } \\
& \text { Non Radioactive } \\
& \text { Non Waste } \\
& \text { Non Haz Substance }\end{array}$ & * & * & & Note 1 & & * & $*$ & * & \\
\hline $\begin{array}{ll}\text { 3. Hazmat } \\
\text { Non Radioactive } \\
\text { Non Waste } \\
\text { Haz Substance }\end{array}$ & * & * & & Note 1 & * & * & * & * & \\
\hline $\begin{array}{ll}\text { 4. } & \text { Hazmat } \\
\text { Radioactive } \\
\text { Non Waste } \\
\text { Non Haz Substance }\end{array}$ & * & * & & Note 1 & & * & * & $*$ & * \\
\hline $\begin{array}{ll}\text { 5. } & \text { Hazmat } \\
\text { Radioactive } \\
\text { Non Waste } \\
\text { Haz Substance }\end{array}$ & * & * & & Note 1 & $*$ & * & * & $*$ & \\
\hline $\begin{array}{l}\text { 6pent Fuel } \\
\text { TRU Waste } \\
\text { Highway Route } \\
\text { Controlled Quantity }\end{array}$ & * & $*$ & & Note 1 & & $*$ & $*$ & $*$ & $*$ \\
\hline
\end{tabular}

${ }^{2}$ International Shipping Operations refers to the international portion of a shipment which may also involve intra- or inter-state shipping segments. For those segments, refer to pages 2-2 - 2-6.

* Applicable agencyiregulation.

** Please see Appendix C for further information and requircments of the Arms Export Control Act (AECA) and the Department of the Treasury's Office of Foreign Assets Control (OFAC).

Note 1 - DOE Orders:

Note 2 - DOE Orders:
$1540.1,1540.2,5480.1 \mathrm{~B}, 5480.3,5480.4,5000.3 \mathrm{~B}, 5633.5$

$1540.1,5000.3 \mathrm{E}, 5480.1 \mathrm{~B}, 5633.5$ 


\begin{tabular}{|c|c|c|c|c|c|c|}
\hline \multicolumn{7}{|c|}{ 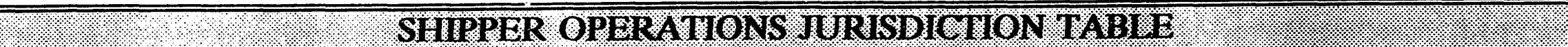 } \\
\hline \multirow[b]{2}{*}{$\begin{array}{c}\text { SHIPPER OPERATIONS } \\
\text { DESCRIPTION }\end{array}$} & \multicolumn{6}{|c|}{ Regulatory Agency/Applicable Regulations } \\
\hline & $\begin{array}{c}\text { RSPA } \\
\text { (HMR) } 49 \text { CFR 171.1 }\end{array}$ & $\begin{array}{c}\text { NRC } \\
10 \text { CFR } 71\end{array}$ & $\begin{array}{l}\text { DOE } \\
\text { Orders }\end{array}$ & $\begin{array}{c}\text { EPA } \\
40 \text { CFR 61, 117, 240-272, } \\
302 \text { (CERCLA) 760-761 }\end{array}$ & $\begin{array}{l}\text { OSHA } \\
29 \text { CFR 1900-1926 }\end{array}$ & $\begin{array}{c}\text { TPSC, TCA Title } \\
65-15-110 \\
65-15-113\end{array}$ \\
\hline \multicolumn{7}{|c|}{ INTERSTATE SHIPPING OPERATIONS } \\
\hline $\begin{array}{ll}\text { 7. Non Hazmat } \\
\text { Non Radioactive } \\
\text { Non Waste } \\
\text { Non Haz Substance }\end{array}$ & $*$ & & Note 2 & & & $*$ \\
\hline $\begin{array}{ll}\text { 8. } & \text { Hazmat } \\
\text { Non Radioactive } \\
\text { Non Waste } \\
\text { Non Haz Substance }\end{array}$ & $*$ & & Note 1 & & * & $*$ \\
\hline $\begin{array}{l}\text { 9. Hazmat } \\
\text { Non Radioactive } \\
\text { Non Waste } \\
\text { Haz Substance }\end{array}$ & * & & Note 1 & $*$ & * & * \\
\hline $\begin{array}{ll}\text { 10. } & \text { Hazmat } \\
\text { Non Radioactive } \\
\text { Waste } \\
\text { Non Haz Substance }\end{array}$ & $*$ & & Note 1 & $*$ & * & * \\
\hline $\begin{array}{l}\text { 11. Hazmat } \\
\text { Non Radioactive } \\
\text { Waste } \\
\text { Haz Substance }\end{array}$ & $*$ & & Note 1 & $*$ & * & $*$ \\
\hline $\begin{array}{ll}\text { 12. } & \text { Hazmat } \\
\text { Radioactive } \\
\text { Non Waste } \\
\text { Non Haz Substance }\end{array}$ & $*$ & $* *$ & Note 1 & & * & $*$ \\
\hline $\begin{array}{ll}13 . & \text { Hazmat } \\
\text { Radioactive } \\
\text { Non Waste } \\
\text { Haz Substance }\end{array}$ & * & $* *$ & Note 1 & $*$ & $*$ & * \\
\hline
\end{tabular}

\section{* Applicable agency/regulation.}

** Applies if shipment is in NRC-certified package.

Note 1 - DOE Orders: $1540.1,1540.2,5480.1 \mathrm{~B}, 5480.3,5480.4,5000.3 \mathrm{~B}$ 


\begin{tabular}{|c|c|c|c|c|c|c|}
\hline \multirow[b]{2}{*}{$\begin{array}{l}\text { SHIPPER OPERATIONS } \\
\text { DESCRIPTION }\end{array}$} & \multicolumn{6}{|c|}{ Regulatory Agency/Applicable Regulations } \\
\hline & $\begin{array}{c}\text { RSPA } \\
\text { (HMR) } 49 \text { CFR 171.1 }\end{array}$ & $\begin{array}{c}\text { NRC } \\
10 \text { CFR } 71\end{array}$ & $\begin{array}{l}\text { DOE } \\
\text { Orders }\end{array}$ & $\begin{array}{c}\text { EPA } \\
\text { 40 CFR 61, 117, 240-272, } \\
\text { 302 (CERCLA) 760-761 }\end{array}$ & $\begin{array}{l}\text { OSHA } \\
29 \text { CFR 1900-1926 }\end{array}$ & $\begin{array}{c}\text { TPSC, TCA Tale } \\
65-15-110 \\
65-15-113 \\
\end{array}$ \\
\hline \multicolumn{7}{|c|}{ INTERSTATE SHIPPING OPERATIONS (CONTINUED) } \\
\hline 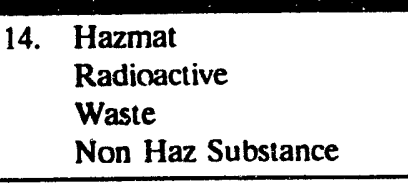 & * & $* *$ & Note 1 & $*$ & $*$ & $*$ \\
\hline $\begin{array}{ll}\text { 15. } & \text { Hazmat } \\
\text { Radioactive } \\
\text { Waste } \\
\text { Haz Substance } \\
\end{array}$ & $*$ & $* *$ & Note 1 & $*$ & $*$ & $*$ \\
\hline $\begin{array}{l}\text { 16. Spent Fuel } \\
\text { TRU Waste } \\
\text { Highway Route } \\
\text { Controlled Quantity }\end{array}$ & $*$ & $*$ & Note 1 & $*$ & * & $*$ \\
\hline \multicolumn{7}{|c|}{ INTRASTATE SHIPPING OPERATIONS } \\
\hline $\begin{array}{l}\text { 17. Non Hazmat } \\
\text { Non Radioactive } \\
\text { Non Waste } \\
\text { Non Haz Substance } \\
\end{array}$ & & & Note 2 & & & $*$ \\
\hline $\begin{array}{ll}18 . & \text { Hazmat } \\
& \text { Non Radioactive } \\
& \text { Non Waste } \\
& \text { Non Haz Substance } \\
\end{array}$ & $*$ & & Note 1 & & $*$ & $*$ \\
\hline $\begin{array}{ll}\text { 19. } & \text { Hazmat } \\
\text { Non Radioactive } \\
\text { Non Waste } \\
\text { Haz Substance }\end{array}$ & $*$ & & Note 1 & $*$ & * & $*$ \\
\hline
\end{tabular}

* Applicable agency/regulation.

** Applies if shipment is in NRC-certified package.

Note 1 - DOE Orders: $1540.1,1540.2,5480.1 \mathrm{~B}, 5480.3,5480.4,5000.313$

Note 2 - DOE Orders: $1540.1,5000.313,5480.1 B$

RFG for TENNESSEE, Page 2-3 


\begin{tabular}{|c|c|c|c|c|c|c|}
\hline \multicolumn{7}{|c|}{ 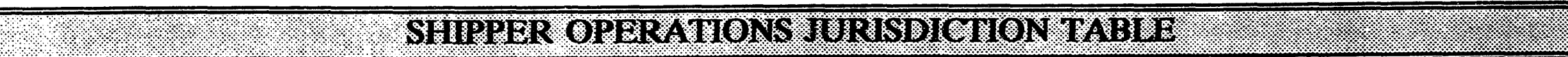 } \\
\hline \multirow[b]{2}{*}{$\begin{array}{l}\text { SHIPPER OPERATIONS } \\
\text { DESCRIPTION }\end{array}$} & \multicolumn{6}{|c|}{ Regulatory Agency/Applicable Regulations } \\
\hline & $\begin{array}{c}\text { RSPA } \\
\text { (HMR) } 49 \text { CFR } 171.1\end{array}$ & $\begin{array}{c}\text { NRC } \\
10 \text { CFR } 71\end{array}$ & $\begin{array}{l}\text { DOE } \\
\text { Orders }\end{array}$ & $\begin{array}{c}\text { EPA } \\
40 \text { CFR 61, 117, 240-272, } \\
302 \text { (CERCLA) 760-761 }\end{array}$ & $\begin{array}{c}\text { OSHA } \\
29 \text { CFR 1900-1926 }\end{array}$ & $\begin{array}{c}\text { TPSC, TCA Title } \\
65-15-110 \\
65-15-113 \\
\end{array}$ \\
\hline $\begin{array}{ll}\text { 20. } & \text { Hazmat } \\
& \text { Non Radioactive } \\
\text { Waste } \\
\text { Non Haz Substance }\end{array}$ & 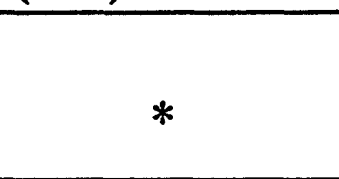 & & Note 1 & $*$ & $*$ & $*$ \\
\hline \multicolumn{7}{|c|}{ INTRASTATE SHIPPING OPERATIONS (CONTINUED) } \\
\hline $\begin{array}{l}\text { 21. Hazmat } \\
\text { Non Radioactive } \\
\text { Waste } \\
\text { Haz Substance }\end{array}$ & $*$ & & Note 1 & $*$ & $*$ & $*$ \\
\hline $\begin{array}{ll}\text { 22. } & \text { Hazmat } \\
& \text { Radioactive } \\
& \text { Non Waste } \\
& \text { Non Haz Substance }\end{array}$ & $*$ & $* *$ & Note 1 & & $*$ & $*$ \\
\hline $\begin{array}{ll}\text { 23. } & \text { Hazmat } \\
\text { Radioactive } \\
\text { Non Waste } \\
\text { Haz Substance }\end{array}$ & $*$ & $* *$ & Note 1 & & $*$ & $*$ \\
\hline $\begin{array}{ll}\text { 24. } & \text { Hazmat } \\
\text { Radioactive } \\
\text { Waste } \\
\text { Non Haz Substance }\end{array}$ & * & $* *$ & Note 1 & $*$ & $*$ & $*$ \\
\hline $\begin{array}{ll}\text { 25. } & \text { Hazmat } \\
\text { Radioactive } \\
\text { Waste } \\
\text { Haz Substance }\end{array}$ & $*$ & $* *$ & Note 1 & $*$ & $*$ & $*$ \\
\hline $\begin{array}{l}\text { 26. Spent Fuel } \\
\text { TRU Waste } \\
\text { Highway Route } \\
\text { Controlled Quantity }\end{array}$ & $*$ & $*$ & Note 1 & $*$ & $*$ & $*$ \\
\hline
\end{tabular}

\section{* Applicable agency/regulation.}

* $\quad$ Applies if shipment is in NRC-certifice package.

Notc 1 - DOE Orders: $1540.1,1540.2,5480.1 \mathrm{~B}, 54 \$ 0.3,5480.4,5000.3 \mathrm{~B}$

Notc 2 - DOE Orders: $1540.1,5000.3 B, 5480.113$ 


\begin{tabular}{|c|c|c|c|c|c|c|}
\hline \multicolumn{7}{|c|}{ 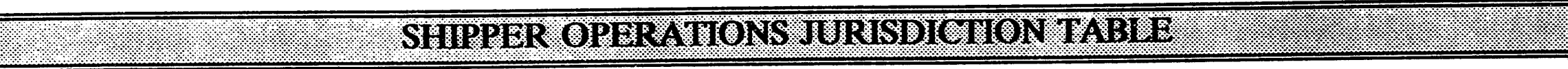 } \\
\hline \multirow[b]{2}{*}{$\begin{array}{l}\text { SHIPPER OPERATIONS } \\
\text { DESCRIPTION }\end{array}$} & \multicolumn{6}{|c|}{ Regulatory Agency/Applicable Regulations } \\
\hline & $\begin{array}{l}\text { RSPA } \\
\text { (HMR) } 49 \text { CFR } 171.1\end{array}$ & $\begin{array}{l}\text { NRC } \\
10 \text { CFR } 71\end{array}$ & $\begin{array}{l}\text { DOE } \\
\text { Orders }\end{array}$ & $\begin{array}{c}\text { EPA } \\
40 \text { CFR 61, 117, 240-272, } \\
302 \text { (CERCLA) 760-761 }\end{array}$ & $\begin{array}{l}\text { OSHA } \\
29 \text { CFR 1900-1926 }\end{array}$ & $\begin{array}{c}\text { TPSC, TCA Tile } \\
65-15-110 \\
65-15-113\end{array}$ \\
\hline $\begin{array}{l}\text { 27. Non Hazmat } \\
\text { Non Radioactive } \\
\text { Non Waste } \\
\text { Non Haz Substance }\end{array}$ & & & Note 2 & & & $*$ \\
\hline $\begin{array}{ll}\text { 28. } & \text { Hazmat } \\
\text { Non Radioactive } \\
\text { Non Waste } \\
\text { Non Haz Substance }\end{array}$ & $*$ & & Note 1 & & $*$ & $*$ \\
\hline $\begin{array}{ll}\text { 29. } & \text { Hazmat } \\
\text { Non Radioactive } \\
\text { Non Waste } \\
\text { Haz Substance } \\
\end{array}$ & $*$ & & Note 1 & $*$ & $*$ & $*$ \\
\hline $\begin{array}{ll}\text { 30. } & \text { Hazmat } \\
\text { Non Radioactive } \\
\text { Waste } \\
\text { Non Haz Substance } \\
\end{array}$ & $*$ & & Note 1 & $*$ & $*$ & $*$ \\
\hline $\begin{array}{ll}\text { 31. } & \text { Hazmat } \\
\text { Non Radioactive } \\
\text { Waste } \\
\text { Haz Substance } \\
\end{array}$ & $*$ & & Note 1 & $*$ & $*$ & * \\
\hline $\begin{array}{ll}\text { 32. } & \text { Hazmat } \\
\text { Radioactive } \\
\text { Non Waste } \\
\text { Non Haz Substance } \\
\end{array}$ & $*$ & $* *$ & Note 1 & & $*$ & $*$ \\
\hline $\begin{array}{ll}\text { 33. } & \text { Hazmat } \\
\text { Radioactive } \\
\text { Non Waste } \\
\text { Haz Substance }\end{array}$ & $*$ & $* *$ & Note 1 & $*$ & $*$ & $*$ \\
\hline
\end{tabular}

* Applicable agency/regulation.

** Applies if shipment is in NRC-certified package.

Note 1 - DOE Orders: $1540.1,1540.2,5480.113,5480.3,5480.4,5000.3 B$

Note 2 - DOE Orders: $1540.1,5000.3 B, 5480.113$

RFG for TENNESSEE, Page 2-5 


\begin{tabular}{|c|c|c|c|c|c|c|}
\hline \multicolumn{7}{|c|}{ 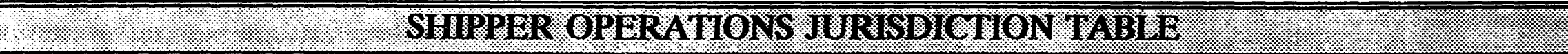 } \\
\hline \multirow[b]{2}{*}{$\begin{array}{l}\text { SHIPPER OPERATIONS } \\
\text { DESCRIPTION }\end{array}$} & \multicolumn{6}{|c|}{ Regulatory Agency/Applicable Regulations } \\
\hline & $\begin{array}{l}\text { RSPA } \\
\text { (HMR) } 49 \text { CFR } 171.1\end{array}$ & $\begin{array}{c}\text { NRC } \\
10 \text { CFR } 71\end{array}$ & $\begin{array}{l}\text { DOE } \\
\text { Orders }\end{array}$ & $\begin{array}{c}\text { EPA } \\
40 \text { CFR 61, 117, 240-272, } \\
302 \text { (CERCLA) 760-761 }\end{array}$ & $\begin{array}{l}\text { OSHA } \\
29 \text { CFR 1900-1926 }\end{array}$ & $\begin{array}{c}\text { TPSC, TCA Title } \\
65-15-110 \\
65-15-113\end{array}$ \\
\hline \multicolumn{7}{|c|}{ ROADS WITHOUT ACCESS CONTROLS SHIPPING OPERATIONS (CONTINUED) } \\
\hline $\begin{array}{ll}\text { 34. } & \text { Hazmat } \\
\text { Radioactive } \\
\text { Waste } \\
\text { Non Haz Substance }\end{array}$ & * & $* *$ & Note 1 & $*$ & * & * \\
\hline $\begin{array}{ll}\text { 35. } & \text { Hazmat } \\
\text { Radioactive } \\
\text { Waste } \\
\text { Haz Substance }\end{array}$ & $*$ & $* *$ & Note 1 & * & $*$ & * \\
\hline $\begin{array}{l}\text { 36. Spent Fuel } \\
\text { TRU Waste } \\
\text { Highway Route } \\
\text { Controlled Quantity }\end{array}$ & * & * & Note 1 & $*$ & * & $*$ \\
\hline
\end{tabular}

* Applicable agency/regulation.

$* * \quad$ Applies if shipment is in NRC-certified package.

Notc 1 - DOE Orders: $1540.1,1540.2,5480.1 \mathrm{~B}, 5480.3,5480.4,5000.3 \mathrm{~B}$ 


\begin{tabular}{|c|c|c|c|c|c|c|c|}
\hline \multicolumn{8}{|c|}{ CARRIRR OPERATIONS TURISDICTION TABIE } \\
\hline \multirow[b]{3}{*}{$\begin{array}{c}\text { CARRIER OPERATIONS } \\
\text { DESCRIPTION }\end{array}$} & \multicolumn{7}{|c|}{ REGULATORY AGENCY/APPLICABLE REGULATIONS } \\
\hline & \multirow{2}{*}{$\begin{array}{c}\text { RSPA } \\
\text { HMR } \\
49 \text { CFR 171.11, } \\
171.15\end{array}$} & \multicolumn{2}{|c|}{ FIIWA } & \multirow{2}{*}{$\begin{array}{c}\text { IPA } \\
40 \text { CFR } \\
240-272\end{array}$} & \multirow{2}{*}{$\begin{array}{c}\text { DOE } \\
\text { DOE } \\
\text { Orders }\end{array}$} & \multirow{2}{*}{$\begin{array}{c}\text { TCA } \\
\text { TPSC Titk } 65\end{array}$} & \multirow{2}{*}{$\begin{array}{c}\text { TCA } \\
\text { TN Dept.Safety } \\
\text { Title 55, Chapter 7- } \\
10,50\end{array}$} \\
\hline & & $\begin{array}{c}\text { FMCSR } \\
49 \text { CFR Part } 383.23\end{array}$ & $\begin{array}{c}\text { FMCSR } \\
49 \text { CFR Part 390- } \\
397\end{array}$ & & & & \\
\hline \multicolumn{8}{|c|}{ INTERSTATE CARRIER OPERATIONS } \\
\hline 1. $\mathrm{CMV}>26 \mathrm{k} \mathrm{lb}$, No Hazmat & * & * & DIR 390.3 & & Note 1 & DIR 65-15-113(a)(2) & DIR \\
\hline 2. CMV > 26k lb, Hazmat & * & * & DIR 390.3 & & Note 1 & DIR $65-15-113(a)(3)$ & DIR \\
\hline 3. $10 \mathrm{k}<\mathrm{CMV}<26 \mathrm{k} \mathrm{lb}$, No Hazmat & & & DIR 390.3 & & Note 2 & DIR 65-15-113(a)(2) & DIR \\
\hline 4. $10 \mathrm{k}<\mathrm{CMV}<26 \mathrm{k} \mathrm{Ib}$, Hazmat & * & & DIR 390.3 & & Note 1 & DIR 65-15-113(a)(3) & DIR \\
\hline 5. $\mathrm{MV}<10 \mathrm{k} \mathrm{Ib}$, Hazmat & * & & PSC & & Note 1 & DIR 65-15-113(a)(3) & DIR \\
\hline 6. Any Placarded Quantity of Hazmat & * & * & DIR 390.3 & & Note 1 & DIR 65-15-113(a)(3) & DIR \\
\hline 7. CMV $>26 \mathrm{k} 16$, Hazardous Waste & * & * & DIR 390.3 & DIR 263.10 & Note 1 & DIR 65-15-113(a)(3) & DIR \\
\hline $\begin{array}{l}\text { 8. } 10 \mathrm{k}<\mathrm{CMV}<26 \mathrm{k} \mathrm{lb} \text {, Hazardous } \\
\text { Waste }\end{array}$ & * & & DIR 390.3 & DIR 263.10 & Note 1 & DIR 65-15-113(a)(3) & DIR \\
\hline 9. MV < 10k lb, Hazardous Waste & $*$ & & PSC & DIR 263.10 & Note 1 & DIR 65-15-113(a)(3) & DIR \\
\hline \multicolumn{8}{|c|}{ INTRASTATE/INTERCITY CARRIER OPERATIONS } \\
\hline 10. CMV > 26k lb, No Hazmat & & * & PSC & & Note 2 & DIR 65-15-113(a)(3) & DIR \\
\hline 11. CMV > 26k lb, Hazmat & * & * & PSC & & Note 1 & DIR 65-15-113(a)(3) & DIR \\
\hline 12. $10 \mathrm{k}<\mathrm{CMV}<26 \mathrm{k} \mathrm{Ib}$, No Hazmat & & & PSC & & Note 2 & DIR $65-15-113(\mathrm{a})(2)$ & DIR \\
\hline 13. $10 \mathrm{k}<\mathrm{CMV}<26 \mathrm{k} \mathrm{lb}$, Hazmat & * & & PSC & & Note 1 & DIR 65-15-113(a)(3) & DIR \\
\hline 14. MV < 10k Ib, Hazmat & * & & PSC & & Note 1 & DIR 65-15-113(a)(3) & DIR \\
\hline
\end{tabular}

* Applicable agency/regulation.

Note 1 - DOIE Orders: $1540.1,1540.2,5480.1 \mathrm{~B}, 5480.3,5480.4,5000.313$

Note 2 - DOE Orders: $1540.1,5000.3 \mathrm{~B}, 5480.1 \mathrm{~B}$ 


\begin{tabular}{|c|c|c|c|c|c|c|c|}
\hline \multicolumn{8}{|c|}{ CARRIER OPERATIONS JURISDICION TABLE } \\
\hline \multirow[b]{3}{*}{$\begin{array}{l}\text { CARRIIER OPIERAITONS } \\
\text { DESCRIPTION }\end{array}$} & \multicolumn{7}{|c|}{ REGULATORY AGENCY/APPLICABLE REGULATIONS } \\
\hline & RSPA & \multicolumn{2}{|c|}{ HIWA } & \multirow{2}{*}{\begin{tabular}{|c|} 
IEPA \\
$40 \mathrm{CFR}$ \\
$240-272$
\end{tabular}} & \multirow{2}{*}{ 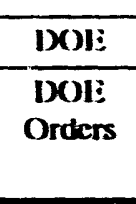 } & \multirow{2}{*}{\begin{tabular}{|c|} 
TCA \\
TPSC Title 65
\end{tabular}} & \multirow{2}{*}{$\begin{array}{c}\text { TCA } \\
\text { IN Depe.Safety } \\
\text { Title 55, Chapter 7- } \\
10,50\end{array}$} \\
\hline & $\begin{array}{c}\text { IIMR } \\
49 \text { CFR 171.11, } \\
171.15\end{array}$ & $\begin{array}{c}\text { FiMCSR } \\
49 \text { CFR Part } 383.23\end{array}$ & $\begin{array}{c}\text { FMMCSR } \\
49 \text { CFR Part 390- } \\
397 \\
\end{array}$ & & & & \\
\hline \multicolumn{8}{|c|}{ INTRASTATE/INTERCITY CARRIER OPERATIONS (CONTINUED) } \\
\hline 15. Any Placarded Quantity of Hazmat & * & $*$ & PSC & & Note 1 & DIR 65-15-113(a)(3) & DIR \\
\hline 16. CMV > 26k lb, Hazardous Waste & * & * & PSC & DIR 263.10 & Note 1 & DIR 65-15-113(a)(3) & DIR \\
\hline 17. $10 \mathrm{k}<\mathrm{CMV}<26 \mathrm{k} \mathrm{tb}$, Haz Waste & * & & PSC & DIR 263.10 & Note 1 & DIR 65-15-113(a)(3) & DIR \\
\hline 18. CMV < 10k lb, Haz Waste & * & & PSC & DIR 263.10 & Note 1 & DIR 65-15-113(a)(3) & $\overline{\text { DIR }}$ \\
\hline \multicolumn{8}{|c|}{ INTRACITY CARRIER OPERATIONS } \\
\hline 19. CMV $>26 \mathrm{k} \mathrm{lb}$, No Hazmat & & * & & & Note 2 & & DIR \\
\hline 20. CMV > 26k lb, Hazmat & * & * & & & Note 1 & & \\
\hline 21. $10 \mathrm{k}>\mathrm{CMV}>26 \mathrm{k} \mathrm{Ib}$, No Hazmat & & * & & & Note 2 & & DIR \\
\hline 22. $10 \mathrm{k}>\mathrm{CMV}>26 \mathrm{k} \mathrm{lb}$, Hazmat & $*$ & * & & & Note 1 & & $\overline{\text { DIR }}$ \\
\hline 23. $M V<10 \mathrm{k}$ lb, Hazmat & $*$ & & & & Note 1 & & DIR \\
\hline 24. Any Placarded Quantity of Hazmat & $*$ & * & & & Note 1 & & DIR \\
\hline 25. CMV > 26k lb, Hazardous Waste & * & * & & DIR 263.10 & Note 1 & & DIR \\
\hline $\begin{array}{l}\text { 26. } 10 \mathrm{k}<\mathrm{CMV}<26 \mathrm{k} \text { lb, Hazardous } \\
\text { Waste }\end{array}$ & * & & & DIR 263.10 & Note 1 & & DIR \\
\hline 27. CMV < 10k tb, Hazardous Waste & * & & & DIR 263.10 & Note 1 & & DIR \\
\hline
\end{tabular}




\begin{tabular}{|c|c|c|c|c|c|c|c|}
\hline \multirow[b]{3}{*}{$\begin{array}{l}\text { CARRIER OPERAIIONS } \\
\text { DESCRIPTION }\end{array}$} & \multicolumn{7}{|c|}{ REGULATORY AGENCY/APPLICABLE REGULATIONS } \\
\hline & \multirow{2}{*}{$\begin{array}{c}\text { KSPA } \\
\text { IMMR } \\
49 \text { CFR } 171.11 \text {, } \\
171.15\end{array}$} & \multicolumn{2}{|c|}{ HIWA } & \multirow{2}{*}{\begin{tabular}{|c|} 
IEPA \\
$40 \mathrm{CFK}$ \\
$240-272$
\end{tabular}} & \multirow{2}{*}{$\begin{array}{l}\text { DOI: } \\
\text { DOE } \\
\text { Orders }\end{array}$} & \multirow{2}{*}{$\frac{\text { TCA }}{\text { TPSC Titke } 65}$} & \multirow{2}{*}{$\begin{array}{c}\text { TCA } \\
\text { TN Dept.Safety } \\
\text { Tite 55, Chapter 7- } \\
10,50\end{array}$} \\
\hline & & $\begin{array}{c}\text { IPMCSR } \\
49 \text { CIRR Part } 383.23\end{array}$ & $\begin{array}{c}\text { FMCSR } \\
49 \text { CFR Part 390- } \\
397\end{array}$ & & & & \\
\hline \multicolumn{8}{|c|}{ RESERVATION ROADS WITHOUT ACCESS RESTRICTION CARRIER OPERATIONS } \\
\hline 28. CMV > 10k lb, No Hazmat & & $*$ & & & Note 2 & & DIR \\
\hline 29. CMV > 10k lb, Hazmat & * & * & & & Note 1 & & DIR \\
\hline 30. $M V<10 \mathrm{k}$ lb, Hazmat & * & & & & Note 1 & & $\overline{\text { DIR }}$ \\
\hline 31. Any Placarded Quantity of Hazmat & * & $*$ & & & Note 1 & & DIR \\
\hline 32. CMV > 26k lb, Hazardous Waste & * & * & & DIR 263.10 & Note 1 & & DIR \\
\hline $\begin{array}{l}\text { 33. 10k < CMV < 26k Ib, Hazardous } \\
\text { Waste }\end{array}$ & * & * & & DIR 263.10 & Note 1 & & DIR \\
\hline 34. CMV < 10k Ib, Hazardous Waste & $*$ & & & DIR 263.10 & Note 1 & & DIR \\
\hline
\end{tabular}





\begin{tabular}{|c|c|c|c|c|c|c|c|c|}
\hline \multicolumn{9}{|c|}{ STATE TRANSTT TABLE } \\
\hline \multirow[b]{2}{*}{ State } & \multicolumn{4}{|c|}{ Permits } & \multirow{2}{*}{$\begin{array}{c}\text { State } \\
\text { Ilaz. Wasle } \\
\text { Manifest }^{2}\end{array}$} & \multirow{2}{*}{$\begin{array}{c}\text { Oversine \& } \\
\text { Overwaight } \\
\text { Contact }\end{array}$} & \multirow{2}{*}{$\begin{array}{l}\text { Governor Designee } \\
\text { For Prenctification } \\
\text { Of Spent Fued }\end{array}$} & \multirow{2}{*}{$\begin{array}{l}\text { NRC } \\
\text { Afreament } \\
\text { State }\end{array}$} \\
\hline & Haomat $^{1}$ & Haz Waste W $^{2}$ & Radioactive' & Spent Fuet ${ }^{3}$ & & & & \\
\hline Alabamas & NR & 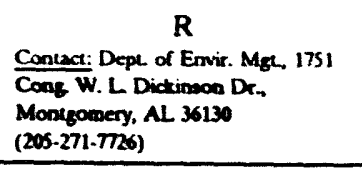 & NR & NR & NR & 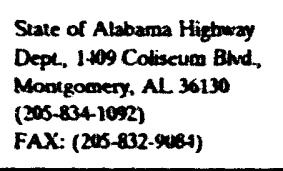 & 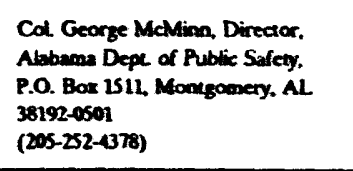 & YES \\
\hline Aleats & NR & 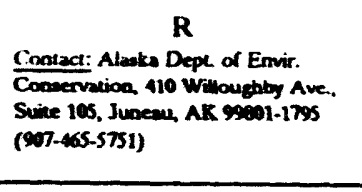 & NR & NR & NR & 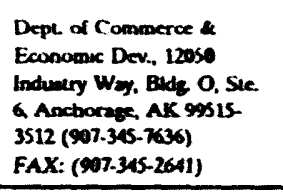 & 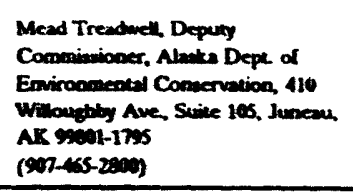 & No \\
\hline Arimona & NR & 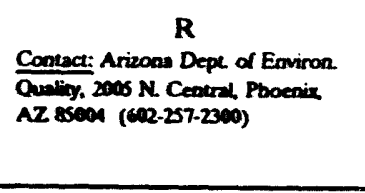 & NR & NR & NR & 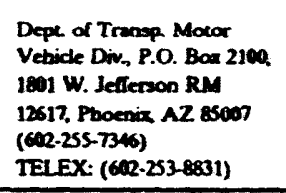 & 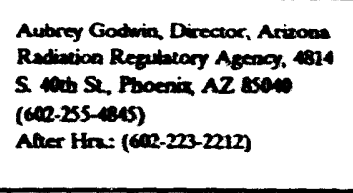 & YES \\
\hline Artameses: & NR & 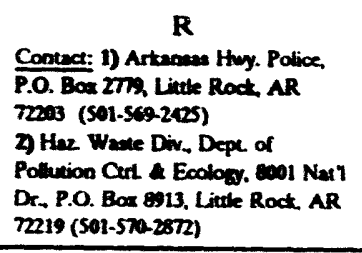 & $\begin{array}{l}\text { NR } \\
\text { Hewever, noufication is } \\
\text { required for rad mal a } \\
\text { high-tevet rad waste. } \\
\text { Conisc: (501-661-2136) }\end{array}$ & NR & 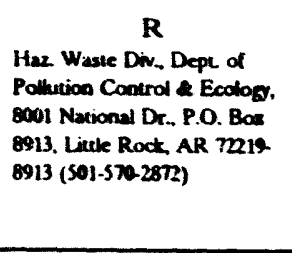 & 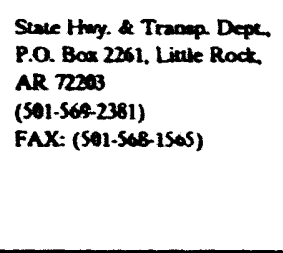 & 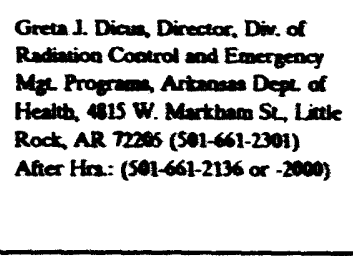 & YES \\
\hline Colitomin & 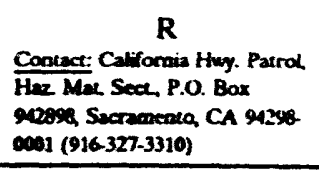 & 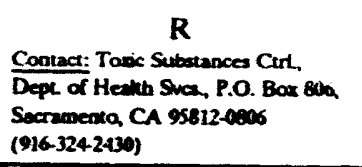 & NR & NR & NR & 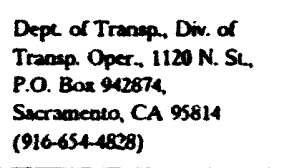 & 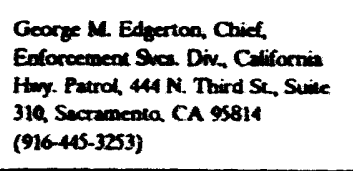 & YES \\
\hline Coloredo & 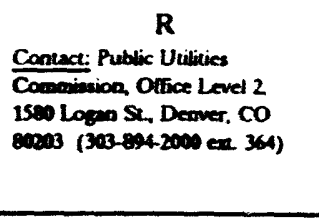 & 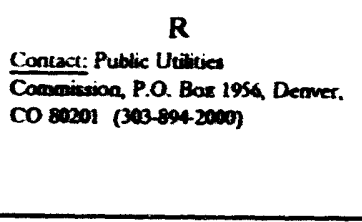 & 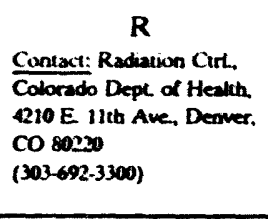 & $\begin{array}{l}\quad R \\
\text { Contast: Colorado } \\
\text { Stace Patrol, } 700 \\
\text { Kipling SL. } \\
\text { Deower, CO 80215 } \\
\text { (303-2290-1595) }\end{array}$ & NR & 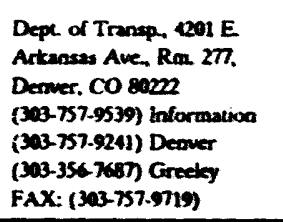 & 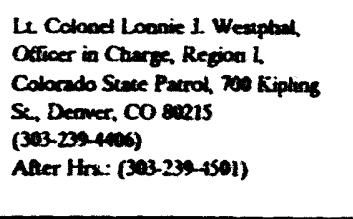 & YES \\
\hline
\end{tabular}




\begin{tabular}{|c|c|c|c|c|c|c|c|c|}
\hline \multicolumn{9}{|c|}{ STATE TRANSIT TABLE } \\
\hline \multirow[b]{2}{*}{ Stave } & \multicolumn{4}{|c|}{ Permits } & \multirow{2}{*}{$\begin{array}{c}\text { State } \\
\text { Ilaz Waste } \\
\text { Manifest }^{2}\end{array}$} & \multirow{2}{*}{$\begin{array}{l}\text { Oversize \& } \\
\text { Ovenweight } \\
\text { Contact }\end{array}$} & \multirow{2}{*}{$\begin{array}{l}\text { Governor Designoe } \\
\text { Por Prenutification } \\
\text { Or Spent Fuets }\end{array}$} & \multirow{2}{*}{$\begin{array}{l}\text { NRC } \\
\text { Afreement } \\
\text { State }\end{array}$} \\
\hline & Harman' & Har Waste ${ }^{2}$ & Radicactive' & Spent Fuet & & & & \\
\hline Comectian & NR & 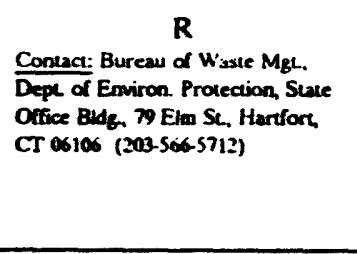 & 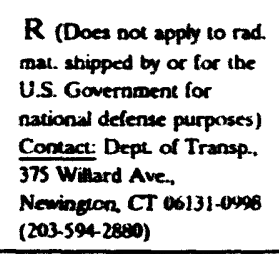 & 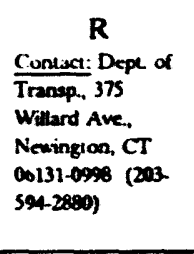 & 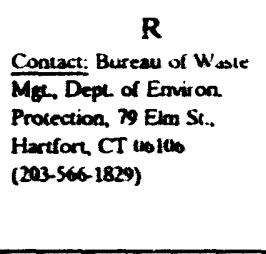 & 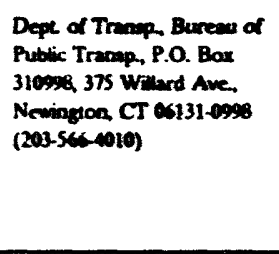 & 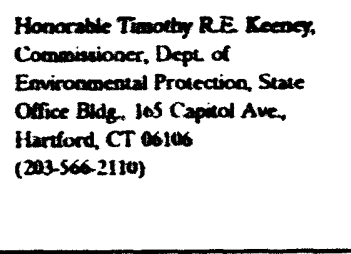 & No \\
\hline Detwerare & 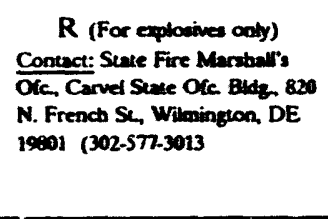 & 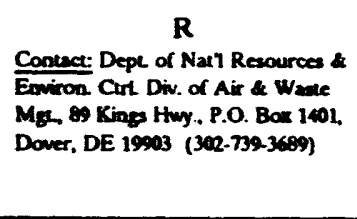 & NR & NR & 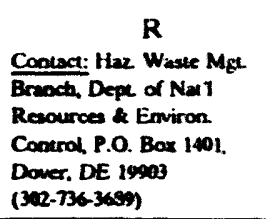 & 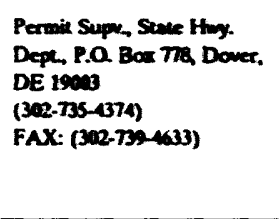 & 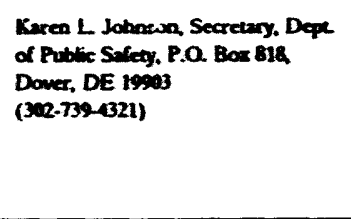 & No \\
\hline $\begin{array}{l}\text { Dintria of } \\
\text { Coturabia }\end{array}$ & 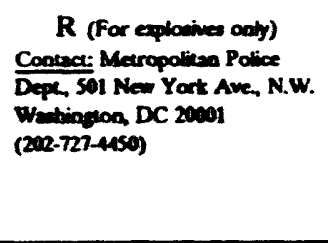 & NR & NR & NR & NR & 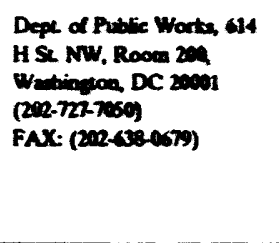 & 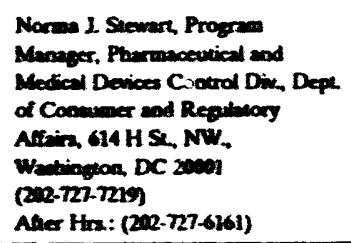 & No \\
\hline Proxith & NR & NR & 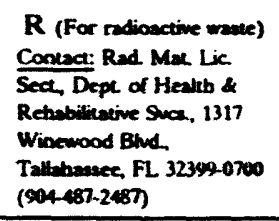 & NR & NR & 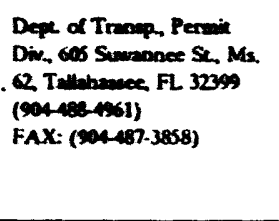 & 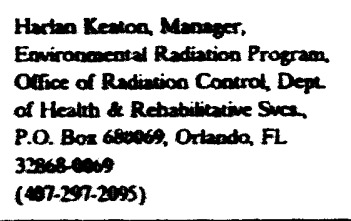 & YES \\
\hline Georga & 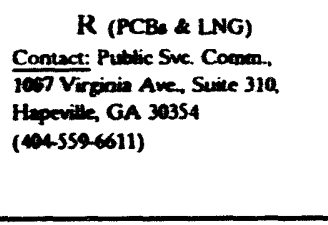 & 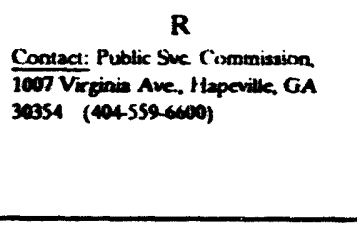 & 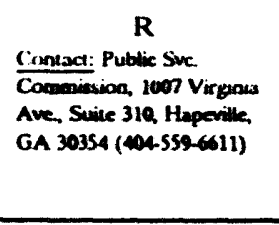 & 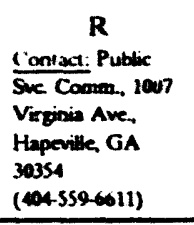 & NR & 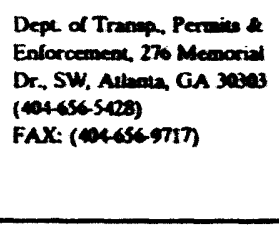 & 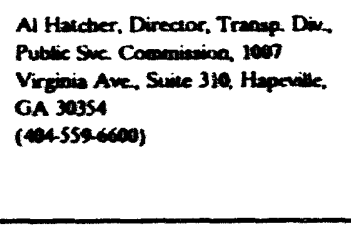 & YES \\
\hline Hami & NR & NR & NR & NR & NR & 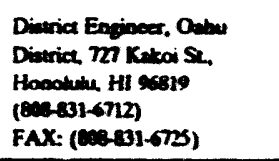 & 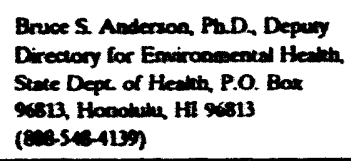 & No \\
\hline
\end{tabular}




\begin{tabular}{|c|c|c|c|c|c|c|c|c|}
\hline \multicolumn{9}{|c|}{ STATE TRANSIT TABLE } \\
\hline \multirow[b]{2}{*}{ State } & \multicolumn{4}{|c|}{ Permits } & \multirow{2}{*}{$\begin{array}{c}\text { State } \\
\text { ILar Waste } \\
\text { Manifest }\end{array}$} & \multirow{2}{*}{$\begin{array}{l}\text { Omervine \& } \\
\text { Omannigh } \\
\text { Contact }\end{array}$} & \multirow{2}{*}{ 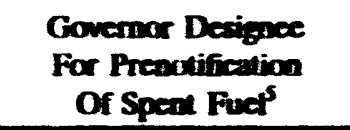 } & \multirow{2}{*}{$\begin{array}{c}\text { NRC } \\
\text { Afreconem } \\
\text { Sinet }\end{array}$} \\
\hline & Hamat ${ }^{1}$ & Har Waste & Radioactive' & Speat Fuer & & & & \\
\hline rato & 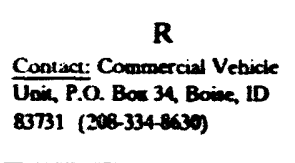 & 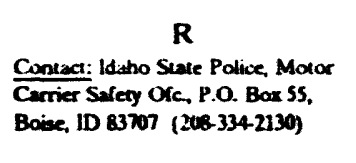 & NR & NR & NR & $\begin{array}{l}\text { Tramep. Depe, P.O. Bor } \\
7129, \text { Buime, 1D 83707.117 } \\
(20.3348420) \\
\text { FAX: (206-3348419) }\end{array}$ & 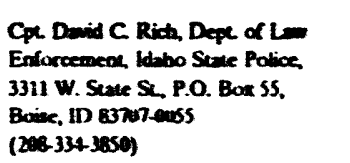 & YES \\
\hline mons & NR & 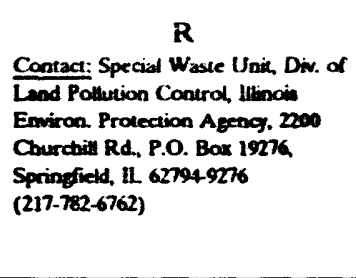 & NR & 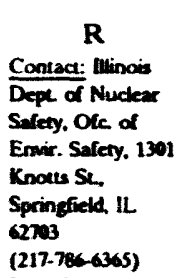 & 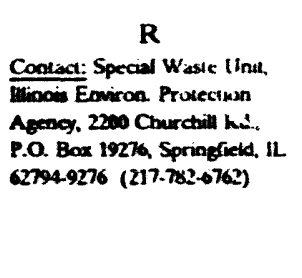 & 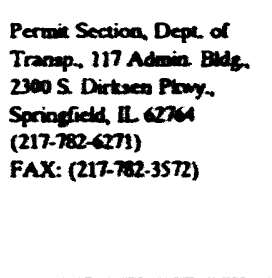 & 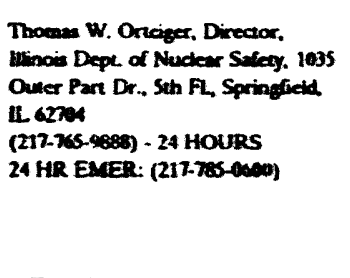 & YES \\
\hline 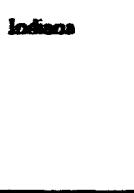 & NR & NR & NR & NR & 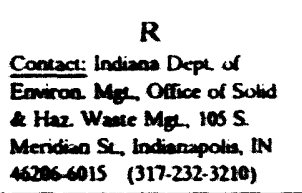 & 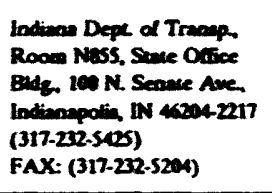 & 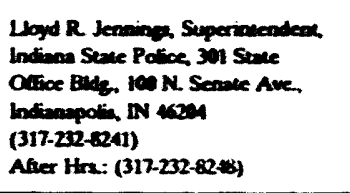 & No \\
\hline 1000 & NR & NR & NR & NR & NR & 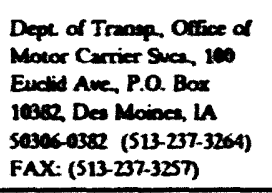 & 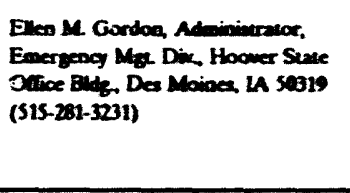 & YES \\
\hline Trmases & NR & 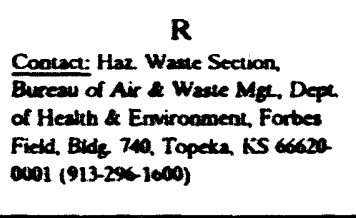 & NR & NR & NR & 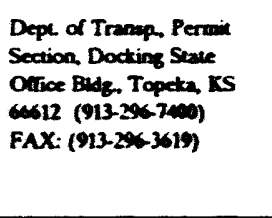 & 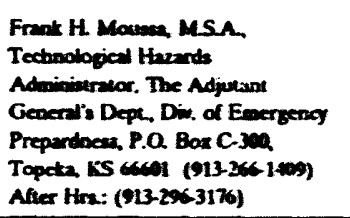 & YES \\
\hline Keneucty & 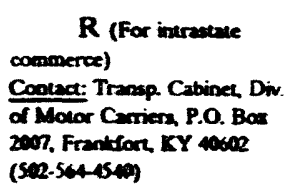 & 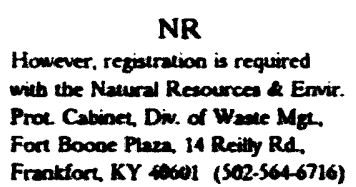 & 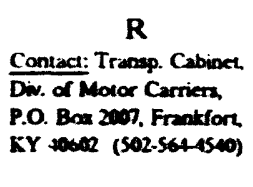 & NR & $\begin{array}{c}\text { NR } \\
\text { (liems D. F. H. L \& K musi be } \\
\text { completed os EPA manifess) }\end{array}$ & 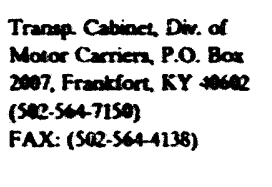 & 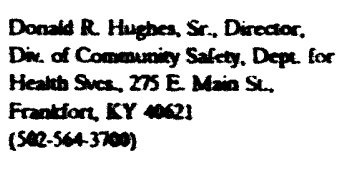 & YES \\
\hline
\end{tabular}




\begin{tabular}{|c|c|c|c|c|c|c|c|c|}
\hline \multicolumn{9}{|c|}{ STATE TRANSIT TABLE } \\
\hline \multirow[b]{2}{*}{ State } & \multicolumn{4}{|c|}{ Permins } & \multirow{2}{*}{$\begin{array}{c}\text { State } \\
\text { Ilez Wastc } \\
\text { Manifest }^{2}\end{array}$} & \multirow{2}{*}{$\begin{array}{l}\text { Oversine \& } \\
\text { Overweight } \\
\text { Contad4 }\end{array}$} & \multirow{2}{*}{$\begin{array}{l}\text { Governor Designce } \\
\text { For Prenuxification } \\
\text { Of Spent Fucts }\end{array}$} & \multirow{2}{*}{$\begin{array}{c}\text { NRC } \\
\text { Afreement } \\
\text { Stance }\end{array}$} \\
\hline & Homant 1 & Har Waste ${ }^{2}$ & Radioactive' & Spent Fuet ${ }^{3}$ & & & & \\
\hline Lovinimas & NR & NR & NR & NR & 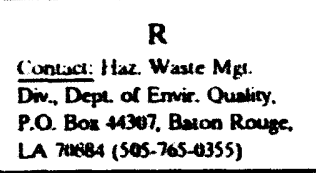 & 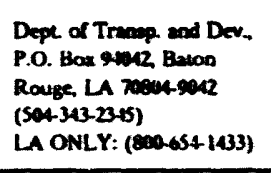 & 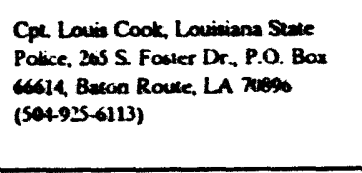 & YES \\
\hline Maine & NR & 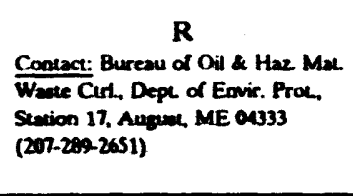 & NR & NR & 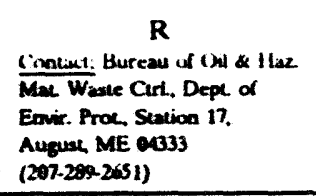 & 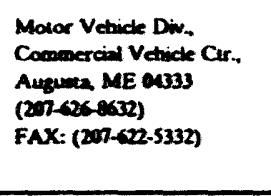 & 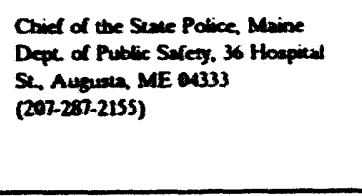 & No \\
\hline Mungtand & NR & 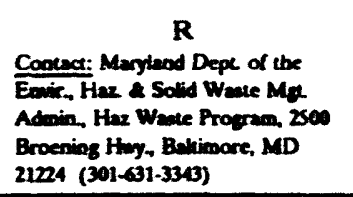 & NR & NR & 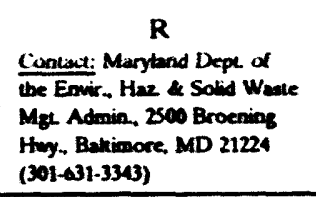 & 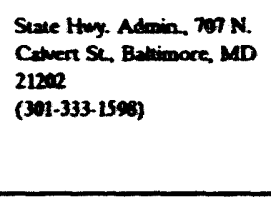 & 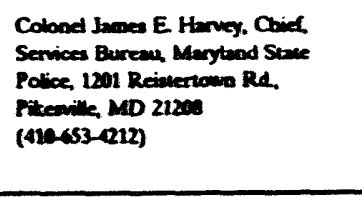 & YES \\
\hline 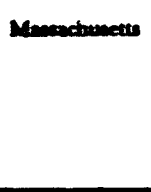 & NR & NR (For Tranoin) & NR & NR & 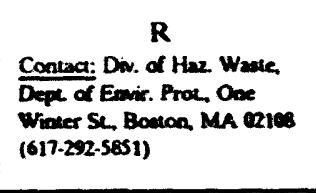 & 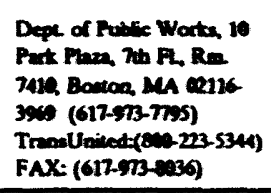 & 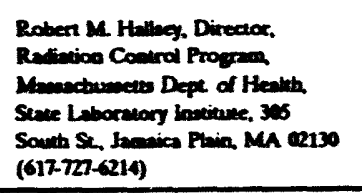 & No \\
\hline Mactiven & 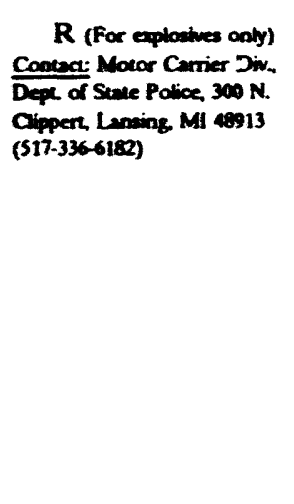 & 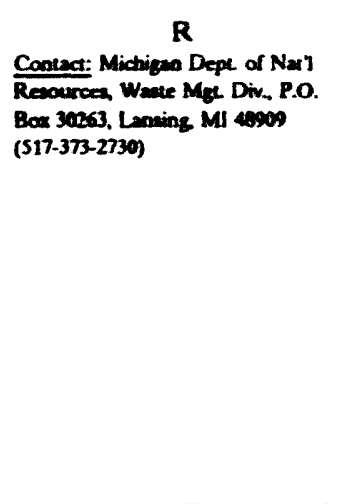 & NR & 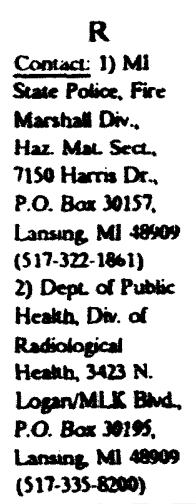 & 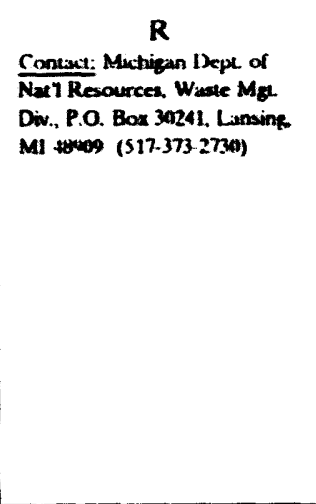 & 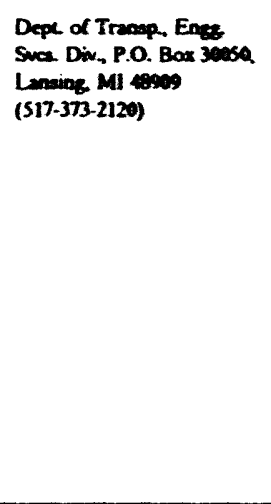 & 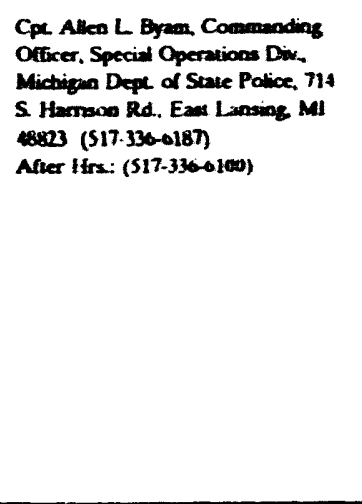 & No \\
\hline
\end{tabular}




\begin{tabular}{|c|c|c|c|c|c|c|c|c|}
\hline \multicolumn{9}{|c|}{ STATE TRANSIT TABLE } \\
\hline \multirow[b]{2}{*}{ State } & \multicolumn{4}{|c|}{ Permits } & \multirow{2}{*}{$\begin{array}{c}\text { State } \\
\text { Ilaz Waste } \\
\text { Manifest }^{2}\end{array}$} & \multirow{2}{*}{$\begin{array}{l}\text { Oversiax \& } \\
\text { Overweight } \\
\text { Contact }\end{array}$} & \multirow{2}{*}{$\begin{array}{l}\text { Governor Designoe } \\
\text { For Prenotification } \\
\text { Of Spene Fuets }\end{array}$} & \multirow{2}{*}{$\begin{array}{l}\text { NRC } \\
\text { Agreenent } \\
\text { State }\end{array}$} \\
\hline & Harman' & Han Waste ${ }^{2}$ & Radioactive' & Spent Fuet 3 & & & & \\
\hline Nimesolen & NR & 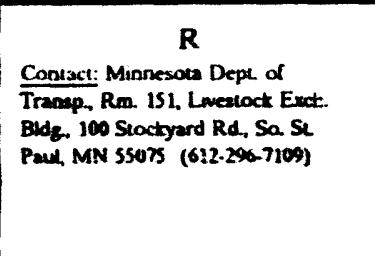 & NR & 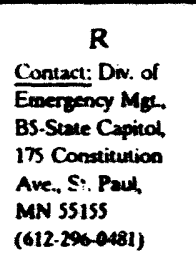 & 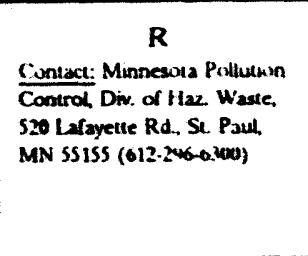 & 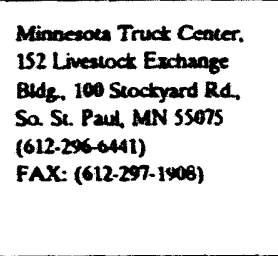 & 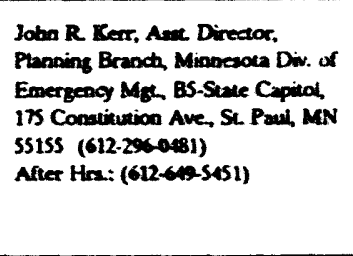 & No \\
\hline Mimineappi & NR & NR & 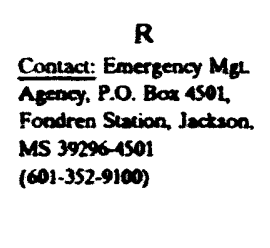 & 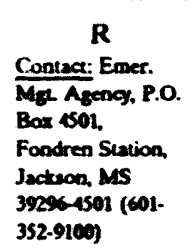 & NR & 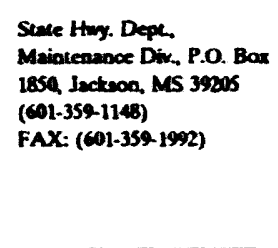 & 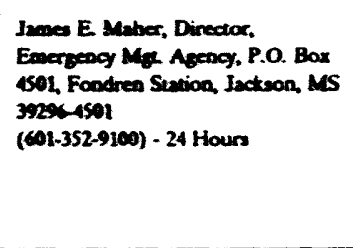 & YES \\
\hline Mimouri & NR & 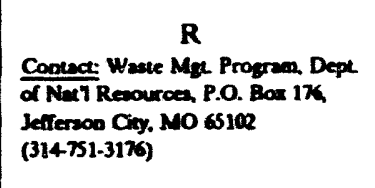 & NR & NR & 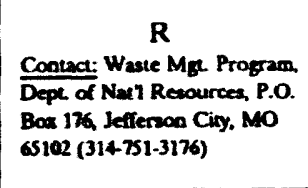 & 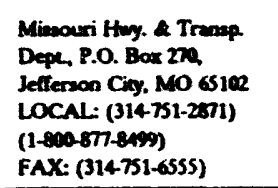 & 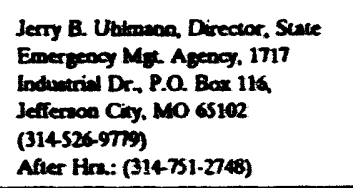 & No \\
\hline Mocemase & NR & NR & NR & NR & NR & 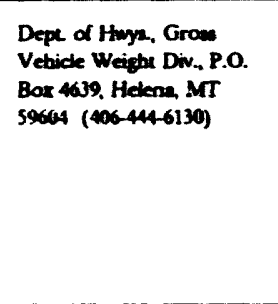 & 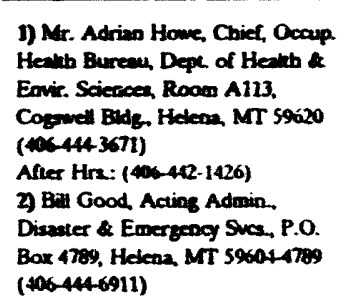 & No \\
\hline Netrents & NR & NR & NR & NR & NR & 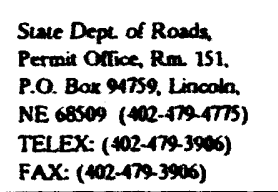 & 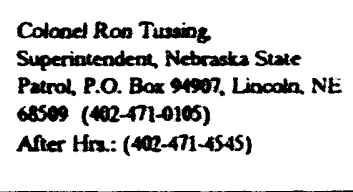 & YES \\
\hline Nonate & 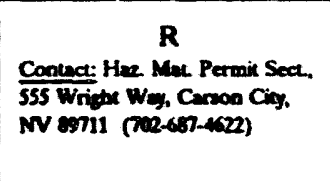 & NR & 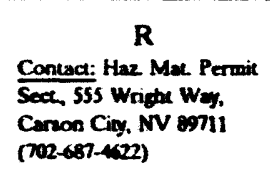 & NR & NR & 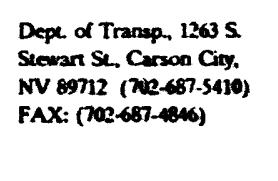 & 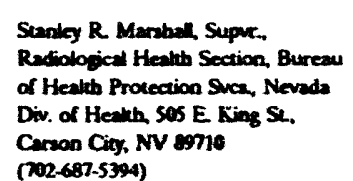 & YES \\
\hline
\end{tabular}




\begin{tabular}{|c|c|c|c|c|c|c|c|c|}
\hline \multicolumn{9}{|c|}{ STATE TRANSIT TABLE } \\
\hline \multirow[b]{2}{*}{ State } & \multicolumn{4}{|c|}{ Permits } & \multirow{2}{*}{$\begin{array}{c}\text { Statc } \\
\text { Itaz Waste } \\
\text { Manifest }\end{array}$} & \multirow{2}{*}{$\begin{array}{l}\text { Oversine \& } \\
\text { Overucight } \\
\text { Contact }\end{array}$} & \multirow{2}{*}{$\begin{array}{l}\text { Governor Designee } \\
\text { For Prenulification } \\
\text { Of Spent Fued }\end{array}$} & \multirow{2}{*}{$\begin{array}{c}\text { NRC } \\
\text { Agrecment } \\
\text { State }\end{array}$} \\
\hline & Harmat ${ }^{1}$ & Haz Waste ${ }^{2}$ & Radioactive ${ }^{t}$ & Spent Fuet ${ }^{3}$ & & & & \\
\hline New & \begin{tabular}{l}
\multicolumn{1}{c}{$\mathbf{R}$} \\
Conlact: Road Toll Admin. \\
Dept of Safey, 10 Hazen Dr., \\
Concord NH 03305 \\
(6003-271.2311)
\end{tabular} & 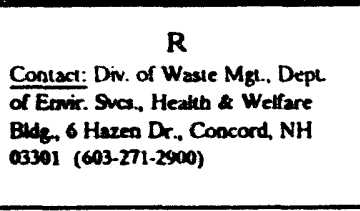 & NR & NR & 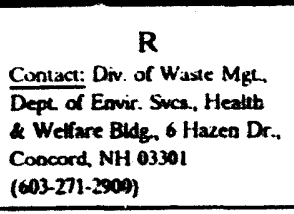 & $\begin{array}{l}\text { Depe, of Transp, they. } \\
\text { Permit Supvr, P.O. Box } \\
483 \text {, Concord, NH 03302. } \\
\text { (4483 (603-271-2691) }\end{array}$ & 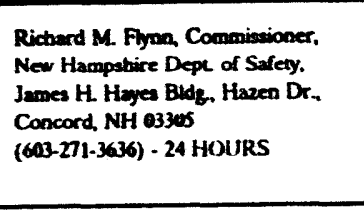 & YES \\
\hline New lersegy & 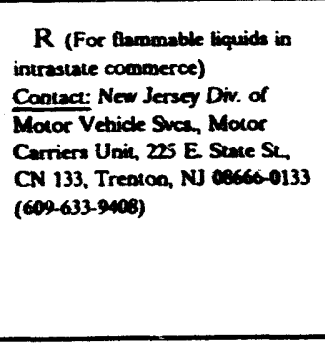 & 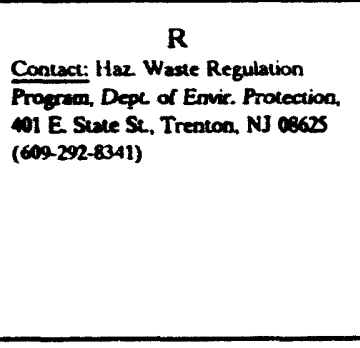 & 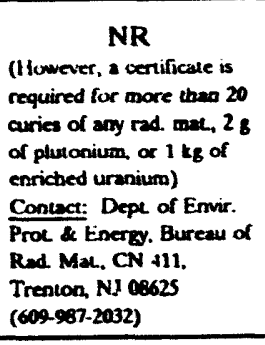 & NR & 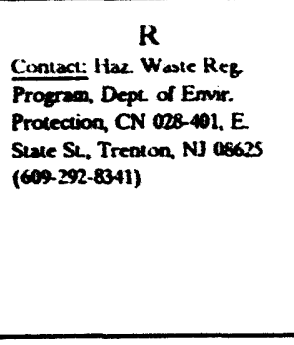 & 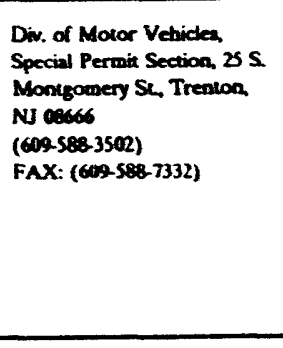 & 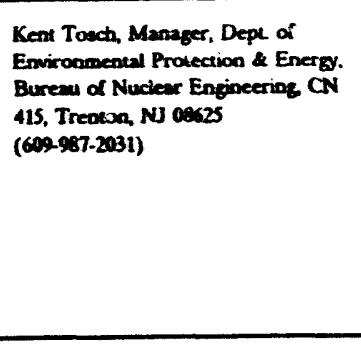 & No \\
\hline New Mesico & NR & 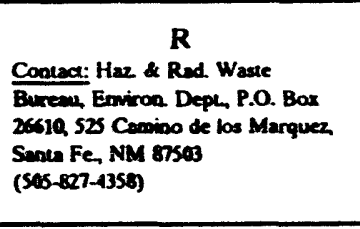 & NR & NR & NR & 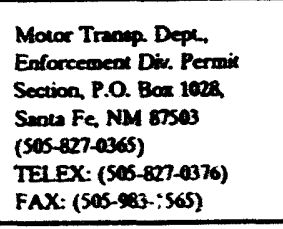 & 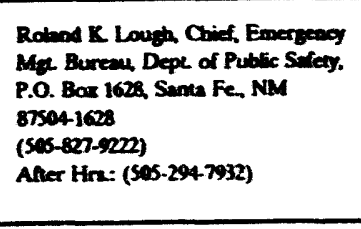 & YES \\
\hline New Yort & NR & 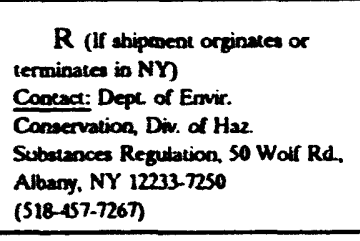 & $\begin{array}{l}\text { R (For LWW } \\
\text { Conlact: Depl of Envir. } \\
\text { Conservation, Div. of Haz } \\
\text { Subatunces Reguation, } 50 \\
\text { Wolf Rd. Albany, NY } \\
\text { 12233-7250 (518-457-7225) }\end{array}$ & NR & NR & 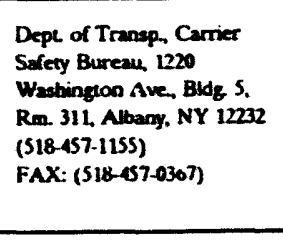 & 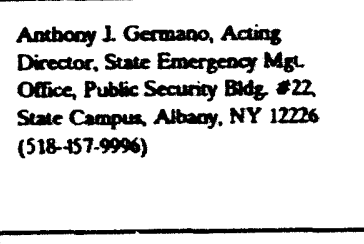 & YES \\
\hline North Cerotina & NR & NR & NR & NR. & $\begin{array}{c}\text { NR } \\
\text { (liems D. F, H, \& I must be } \\
\text { completed on EPA manifea) }\end{array}$ & 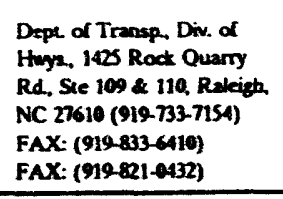 & 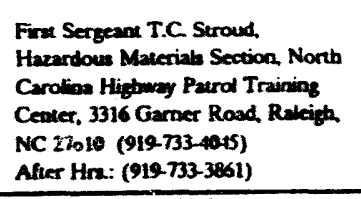 & YES \\
\hline North Datoon & 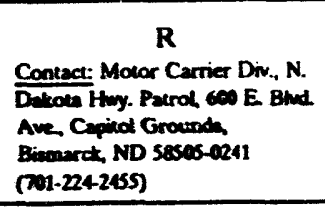 & 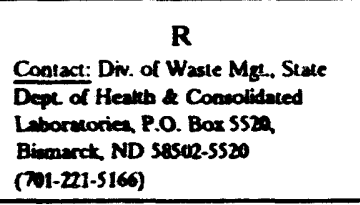 & NR & NR & NR & 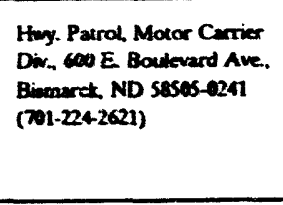 & 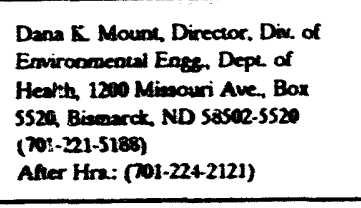 & YES \\
\hline
\end{tabular}




\begin{tabular}{|c|c|c|c|c|c|c|c|c|}
\hline \multicolumn{9}{|c|}{ STATE TRANSIT TABLE } \\
\hline \multirow[b]{2}{*}{ Sinte } & \multicolumn{4}{|c|}{ Permits } & \multirow{2}{*}{$\begin{array}{c}\text { Statc } \\
\text { Ilazz Waste } \\
\text { Manifest }^{2}\end{array}$} & \multirow{2}{*}{$\begin{array}{l}\text { Oversine \& } \\
\text { Cwerweight } \\
\text { Contact }\end{array}$} & \multirow{2}{*}{$\begin{array}{l}\text { Governor Designee } \\
\text { I"or Premutification } \\
\text { Of Spent Fuet }\end{array}$} & \multirow{2}{*}{$\begin{array}{l}\text { NRC } \\
\text { Agreement } \\
\text { State }\end{array}$} \\
\hline & Harmat ${ }^{1}$ & Haz Waste ${ }^{2}$ & Radioactive' & Spent Fuer? & & & & \\
\hline OAso & \begin{tabular}{l}
\multicolumn{1}{c}{$R$} \\
Conlect: Public Utilities \\
Commainsion, 180 E. Broad St. \\
Cohumbur OH 43266-0573 \\
(614-466-7232)
\end{tabular} & \begin{tabular}{l}
\multicolumn{1}{c}{$R$} \\
Contect: Public Utilities \\
Comminarion, 189 E Brosad SL. \\
Columbur, OH 43266-0573 \\
(614.+66-0109)
\end{tabular} & NR & NR & $\begin{array}{l}\text { NR } \\
\text { (Ilem I must be cumpleted on } \\
\text { EPA manif(ssi) }\end{array}$ & 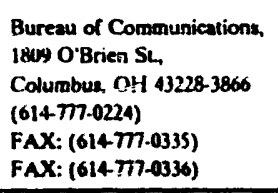 & 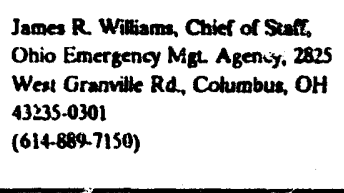 & No \\
\hline Otlehome & NR & 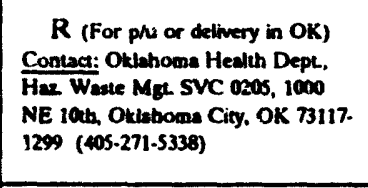 & NR & NR & NR & $\begin{array}{l}\text { Depl of Public Safery, Size } \\
\text { \& Weight Permit Div, P.O. } \\
\text { Bax 11415, Otlaboma Ciry, } \\
\text { OK 73136-0115 } \\
\text { (405-125-2390) } \\
\text { FAX: (405-125-2811) }\end{array}$ & 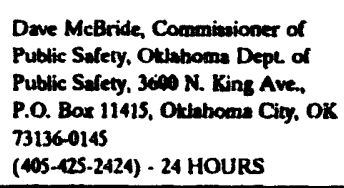 & No \\
\hline Oreson & NR & 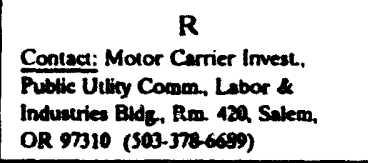 & $\begin{array}{c}\mathbf{R} \\
\text { Cinntact: Tranap. Sufery } \\
\text { Diw., Public Utiliy Comm. } \\
\text { 350 Winter SL, NE, Salem. } \\
\text { OR } 97310 \text { (503-378-5916) }\end{array}$ & NR & $\begin{array}{l}\text { NR } \\
\text { (Requires additional stale } \\
\text { information on EPA manifest) }\end{array}$ & $\begin{array}{l}\text { Transp. Permit Uniil, } 2950 \\
\text { State St., Rm. } 216 \text { Salem. } \\
\text { OR } 97310 \\
\text { (503-378-2568) } \\
\text { FAX: }(503-373-737)\end{array}$ & $\begin{array}{l}\text { Dewid Stewart-Smith, Adminizurator, } \\
\text { Fadity Regulation Div, Oregon } \\
\text { Deph, of Energs. } 625 \text { Marion SC. } \\
\text { NE, Solem, OR } 97310 \\
\text { (503-378-6169) }\end{array}$ & YES \\
\hline Pemogyanis & 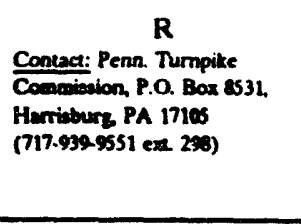 & 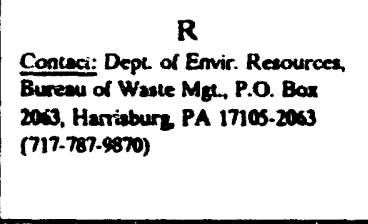 & NR & 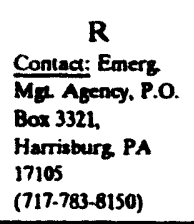 & \begin{tabular}{l}
\multicolumn{1}{c}{$\mathbf{R}$} \\
Conlact: Dept of Einvir. \\
Resourcea, Bureau of Waste \\
Mg., P.O. Box 2063. \\
Harrisburg PA 17105-2063 \\
(717.787.9870)
\end{tabular} & $\begin{array}{l}\text { Depl. of Transp. Central } \\
\text { Permit Ofc, Rm 1014, } \\
\text { Transp. \& Sity. Bldg. } \\
\text { Harrisburg, PA 17120 } \\
\text { (717-787.-4600) } \\
\text { FAX: (717.787.9690) }\end{array}$ & 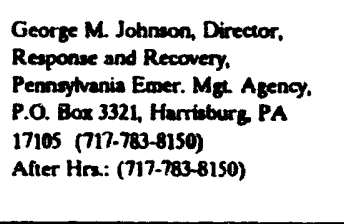 & No \\
\hline Rbode lalund & NR & \begin{tabular}{l}
\multicolumn{1}{c}{$\mathbf{R}$} \\
Contect: Div. of Air \& Haz. MaL. \\
Deph. of Envir. MgL. 291 \\
Promenede Sc. Providence, RI 02908 \\
(\$01-277.2797)
\end{tabular} & $\begin{array}{l}\text { R (Does not apply to rad. } \\
\text { mat. shipped by or for the } \\
\text { U.S. Gov' for national } \\
\text { defense purposes) } \\
\text { Consact: Public Ullities } \\
\text { Comm.. 100 Orange St. } \\
\text { Providence, RI } 02903 \\
(401-277.3500)\end{array}$ & NR & 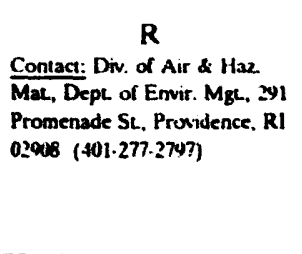 & $\begin{array}{l}\text { Depl of Transp. Mocor } \\
\text { Vethick Div., Stute Office } \\
\text { Bldg. Rm. } 106 \text {. Permits, } \\
\text { Providence, RI 02903 } \\
\text { (\$01-277.2986) }\end{array}$ & 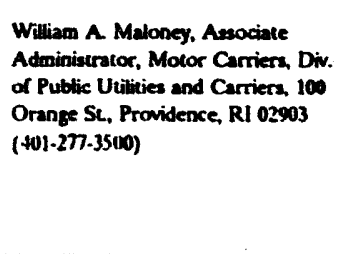 & YES \\
\hline South Carolina & 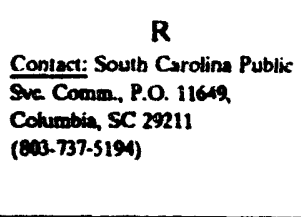 & 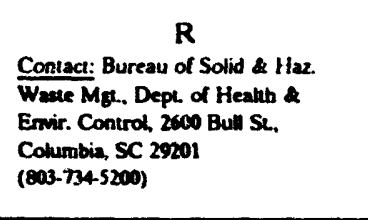 & NR & NR & $\begin{array}{l}\quad \mathrm{R} \\
\text { Contact: Burcau of Solid \& } \\
\text { Haz Waste MgL. Depl of } \\
\text { Health \& Emvir. Control, } 2600 \\
\text { Bull SL. Columbia. SC 29201 } \\
\text { (803-734-S300) }\end{array}$ & $\begin{array}{l}\text { Suate Hwy. Deph, Overnize } \\
\text { Permit Section, P.O. Bax } \\
\text { 191, Annex 3, Columbia, SC } \\
\text { 29202 (803-737.1279) }\end{array}$ & 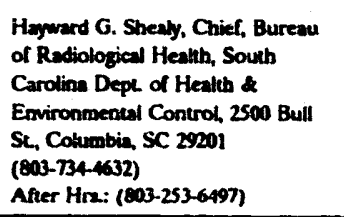 & YES \\
\hline
\end{tabular}




\begin{tabular}{|c|c|c|c|c|c|c|c|c|}
\hline \multicolumn{9}{|c|}{ STATE TRANSIT TABLE } \\
\hline \multirow[b]{2}{*}{ State } & \multicolumn{4}{|c|}{ Permits } & \multirow{2}{*}{$\begin{array}{c}\text { Statc } \\
\text { Ilaz Waste } \\
\text { Manifest }^{2}\end{array}$} & \multirow{2}{*}{$\begin{array}{l}\text { Oversize \& } \\
\text { Overweight } \\
\text { Contact }\end{array}$} & \multirow{2}{*}{$\begin{array}{l}\text { Governor Designoe } \\
\text { For Prenotification } \\
\text { Of Spent Fuels }\end{array}$} & \multirow{2}{*}{$\begin{array}{c}\text { NRC } \\
\text { Agreement } \\
\text { State }\end{array}$} \\
\hline & Harmat' & Haz Waste ${ }^{2}$ & Radioactive' & Spent Fuet ${ }^{3}$ & & & & \\
\hline Sounh Dakots & NR & NR & NR & NR & NR & 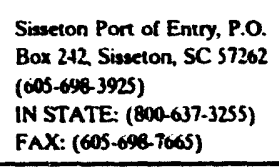 & 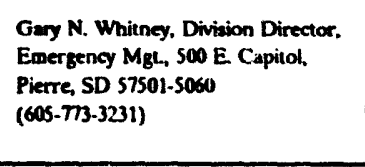 & No \\
\hline Tennesvee & NR & 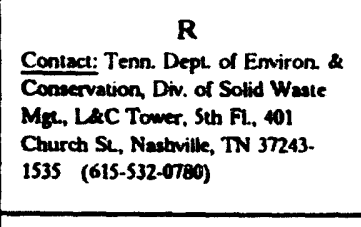 & NR & NR & NR & 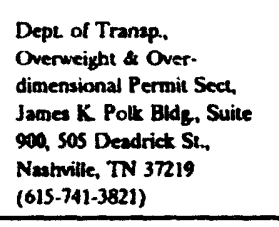 & 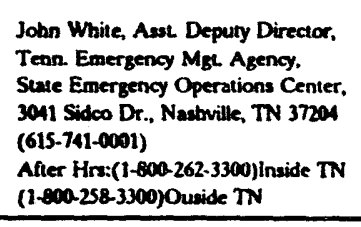 & YES \\
\hline Temon & NR & 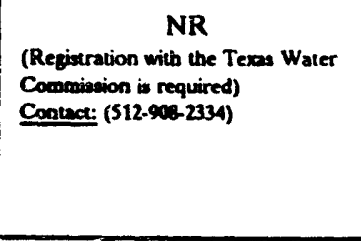 & NR & NR & 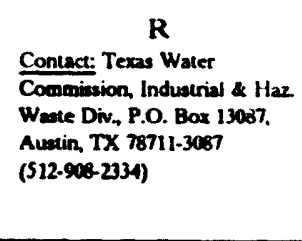 & 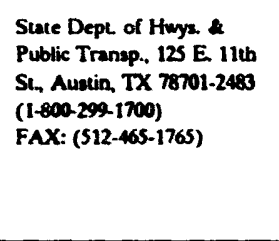 & 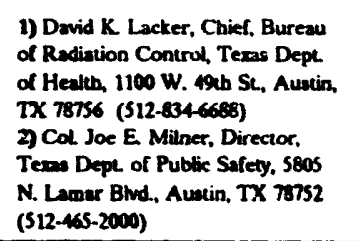 & YES \\
\hline Uater & NR & NR & NR & NR & NR & 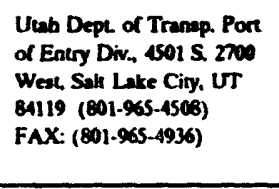 & 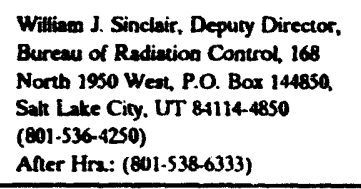 & YES \\
\hline Vermont & 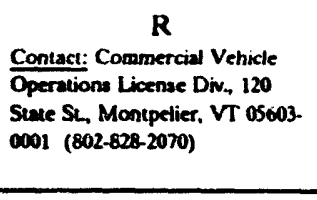 & 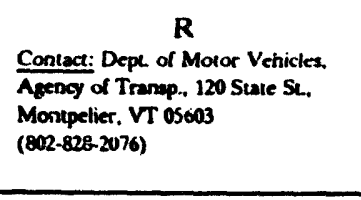 & NR & NR & 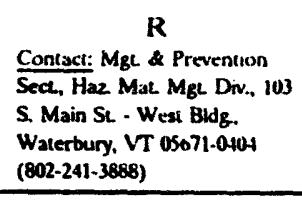 & 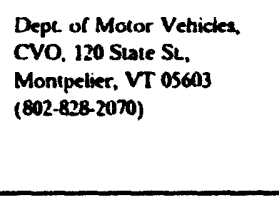 & 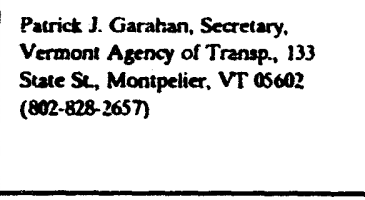 & No \\
\hline Virgenin & NR & 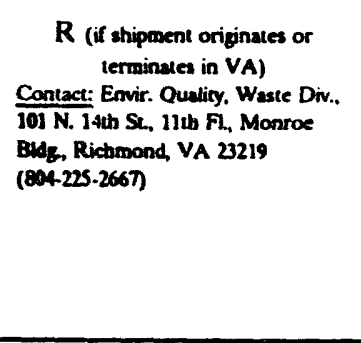 & NR & 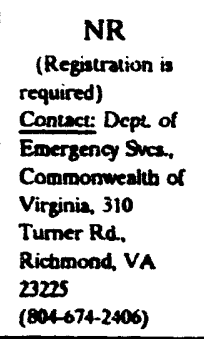 & NR & 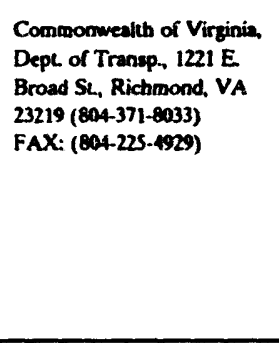 & 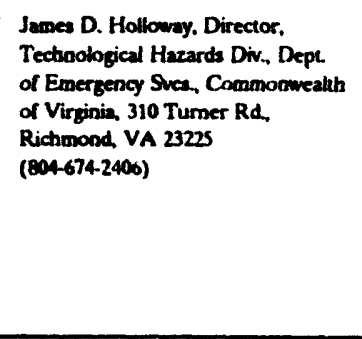 & No \\
\hline
\end{tabular}




\begin{tabular}{|c|c|c|c|c|c|c|c|c|}
\hline \multicolumn{9}{|c|}{ STATE TRANSIT TABLE } \\
\hline \multirow[b]{2}{*}{ State } & \multicolumn{4}{|c|}{ Permits } & \multirow{2}{*}{$\begin{array}{c}\text { State } \\
\text { Ilaz. Waste } \\
\text { Manifest }^{2}\end{array}$} & \multirow{2}{*}{$\begin{array}{l}\text { Oversize \& } \\
\text { Overweight } \\
\text { Contact }\end{array}$} & \multirow{2}{*}{$\begin{array}{l}\text { Governor Designee } \\
\text { For Prenotification } \\
\text { Of Spent Fuet }\end{array}$} & \multirow{2}{*}{$\begin{array}{l}\text { NRC } \\
\text { Agreement } \\
\text { State }^{6}\end{array}$} \\
\hline & Harmal ${ }^{1}$ & Har Waste ${ }^{2}$ & Radioactive & Spent Fuel & & & & \\
\hline Wahington & NR & NR & NR & NR & $\begin{array}{c}\text { NR } \\
\text { (llems D. F. H. \& I must he } \\
\text { completed on EPA manifest) }\end{array}$ & $\begin{array}{l}\text { Depl of Transp., Motor } \\
\text { Carricr Safety Swc, P.O. } \\
\text { Box 47367, OHympia, WA } \\
\text { 98504-7367 (206-159-6715) } \\
\text { FAX: (206-438-7163) } \\
\end{array}$ & 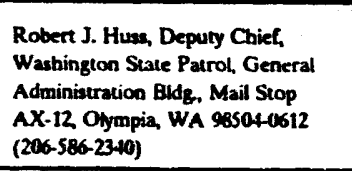 & YES \\
\hline Wear Virginis & NR & NR & NR & NR & NR & 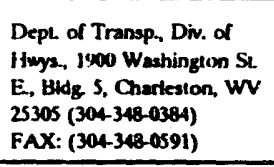 & $\begin{array}{l}\text { Cot. J.R. Buckalew, Superintendent } \\
\text { Deph. of Public Sadey, } 725 \text { Jefferson } \\
\text { Rd. South Charleston, WV 25309 } \\
\text { (304-746-2111) }\end{array}$ & NO \\
\hline Wisconsin & 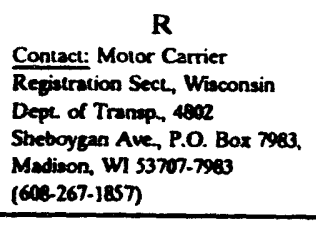 & $\begin{array}{l}\text { R } \\
\text { Conlact: Haz. Waste MgL Section, } \\
\text { Bureau of Solid \& Haz. Waste Mgt. } \\
\text { Depe of Nat7 Reaurces, P.O. Box } \\
\text { 7921, Madison, Wh 53707.7921 } \\
\text { (600-266-2111) }\end{array}$ & $\begin{array}{l}\text { R } \\
\text { Contacl: WI Div. of Motor } \\
\text { Veticles, P.O. Box 7955s } \\
\text { Madian, WI S3707.7955 } \\
\text { (608-267-6764) }\end{array}$ & NR & 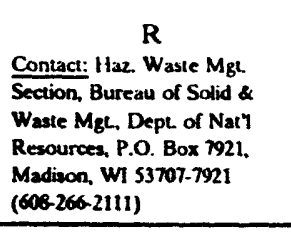 & $\begin{array}{l}\text { Dept. of Transp., Permits } \\
\text { Unit, P.O. Bax } 7989, \\
\text { Madison, W/ } 53707.7980 \\
\text { (608-266-7320) }\end{array}$ & $\begin{array}{l}\text { Leroy E Conner, Jr., Administrator. } \\
\text { Wisconsin Din. of Emergency } \\
\text { Govr., P. O. Box 7865. Madizon, W1 } \\
\text { S3707-7865 } \\
\text { (606-266-3232) }\end{array}$ & NO \\
\hline Wyoming & NR & NR & $\begin{array}{l}R \text { (For radioactive waste) } \\
\text { Contuct: Wyoming Hwy. } \\
\text { Patrot, P. O. Box } 1708 \\
\text { Cheyenne, WY } 82003-1708 \\
\text { (307.77.4317) }\end{array}$ & $\begin{array}{l}\quad \mathbf{R} \\
\text { Contact: The port } \\
\text { of entry that will } \\
\text { be used. Call the } \\
\text { WY Hwy. Patrol } \\
\text { for more info. }\end{array}$ & NR & $\begin{array}{l}\text { Size Permitz-information, } \\
\text { Wyoming ituy. Patrol, P.O. } \\
\text { Box } 1708 \text { Cbeyenne, WY } \\
82003-1708 \\
\text { (307-m-1317) } \\
\text { FAX: (307-m-4282) }\end{array}$ & 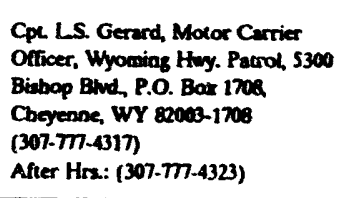 & No \\
\hline
\end{tabular}

1]. J. Keller \& Associates, Inc., State Hazardous Materials Manual: State Requirements for Shipping and Transponation, Neenah, Wl, 1993.

2J. J. Keller \& Associates, Inc., Hazardous Waste Regulatory Guide: State Waste Managernem Programs (Generutor, Transponter, Treameent, Starage \& Disposal Standards), Neenah, WI, 1993.

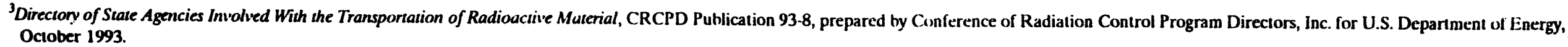

4Size and Weight Updase 1993, prepared by GO-West magazine and the Western Highway Institute for Kenworth Trucking Company and TIP Transport International Pool.

SFederal Register, Vol. 58, No. 124, p. 35050, Government Printing Office, Washington, DC, June 30, 1993.

${ }^{6}$ Richard Woodruff, Agreement Officer, NRC Region II, Allanta, GA (404-331-5553). 


\begin{tabular}{|c|c|c|c|c|}
\hline \multicolumn{5}{|c|}{ TRIBAL TRANSIT TABLE } \\
\hline \multirow{2}{*}{ TIRIBAL INITIYY } & \multirow{2}{*}{ STAITES INVOI.VIID } & \multicolumn{3}{|c|}{ TRIBAI. CONTACT } \\
\hline & & TTHE & NAME: & ADDRESSPHONE NUMBER \\
\hline Eastern - Cherokee & NC & Principal & Jonathan L. Tayior & $\begin{array}{l}\text { P.O. Box } \\
\text { Cherokee, NC } 28719 \\
(704-497-2770) \text { FAX: }(704-497-2950)\end{array}$ \\
\hline Hopi & $A Z$ & Chairman & Vernon Masayesva & $\begin{array}{l}\text { P.O. Box } \\
\text { Kykotsmovi, AZ 86039 } \\
(602-734-2440) \text { FAX: }(602-734-2430)\end{array}$ \\
\hline Jicarilla & NM & President & Levi Pesata & $\begin{array}{l}\text { P.O. Box } \\
\text { Dulce, NM 87528 } \\
(505-759-3240) \text { FAX: }(505-759-3000)\end{array}$ \\
\hline Navajo & $A Z, N M, U T$ & President & Petersen Zah & $\begin{array}{l}\text { P.O. Box } \\
\text { Window Rock, AZ } 86515 \\
\text { (602-871-6350) FAX: }(602-871-4020)\end{array}$ \\
\hline Ramah & NM & President & Martha Garcia & $\begin{array}{l}\text { Rt. 2, Box } \\
\text { Ramah, NM 87321 } \\
(505-775-3380) \text { FAX: }(505-775-3550)\end{array}$ \\
\hline
\end{tabular}

DOE/EM-1 has committed to provide advance notice of DOE spent fuel and high-level waste shipments to Indian tribes whose jurisdictions are crossed by or adjacent to transportation routes.

NOTE: This is an example of what ultimately will be provided. Information is being made available from the Battelle Seattle Research Center. Approximately 546 tribal contacts have been identified and will be included in future updates. Also, it is envisioned that a map showing the boundaries of all federally-recognized tribal entities within a state will be provided for each state. 


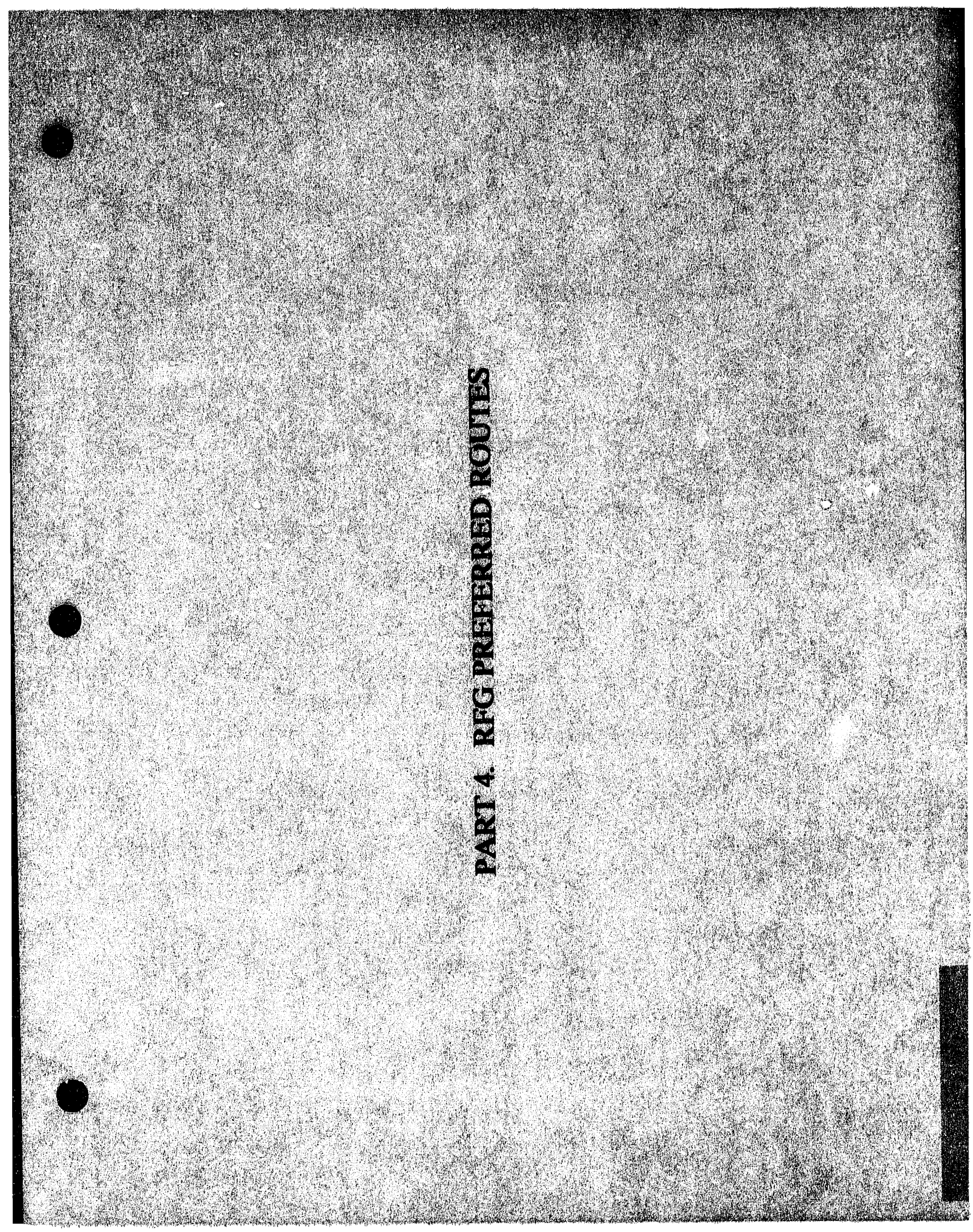




\section{Definition of Preferred Routes}

49 CFR 177.825 establishes the criterion for preferred routes. "A carrier or any person operating a motor vehicle containing a highway route controlled quantity of Class 7 (radioactive) materials, as defined in 49 CFR 173.403(1), shall operate the motor vehicle only over preferred routes." Preferred routes consist of Interstate System highways. If an Interstate System bypass or Interstate System beltway exists around a city, these roads shall be used in place of the Interstate System highway through the city. States routing agencies have the option of designating alternative routes, in addition to, or in lieu of, Interstate System routes.

Routes must be selected to reduce the time in transit over the preferred route segment of the trip. For pick-up and delivery not over preferred routes, the route selected must be the shortest-distance route from the pick-up location to the nearest preferred route entry location, and the shortest-distance from the nearest preferred route exit location.

The following list identifies route segments which meet the 49 CFR 177.825 definition of preferred routes for the identified state. Following the list of preferred routes is a list of the State-designated routes. 


\section{Preferred Routes in Alabama}

1-10 - From the Mississippi state line to Florida state line

1-20 - From the junction with 1-459 east of Birmingham to the Georgia state line

I-20/I-59 - From Mississippi state line to the junction with I-65 in Birmingham

I-459 - From the junction with I-20/1-59 southwest of Birmingham to the junction with 1-59 northeast of Birmingham

1-565 - From the junction with 1-65 northeast of Decatur to Huntsville

I-59 From the junction with 1-459 northeast of Birmingham to the Georgia state line

I-65 - From the junction with I-10 southwest of Mobile to the Tennessee state line

1-85 - From the junction with 1-65 in Montgomery to the Georgia state line

\section{State Designated Preferred Routes}

Alabama has not designated any additional roads. 


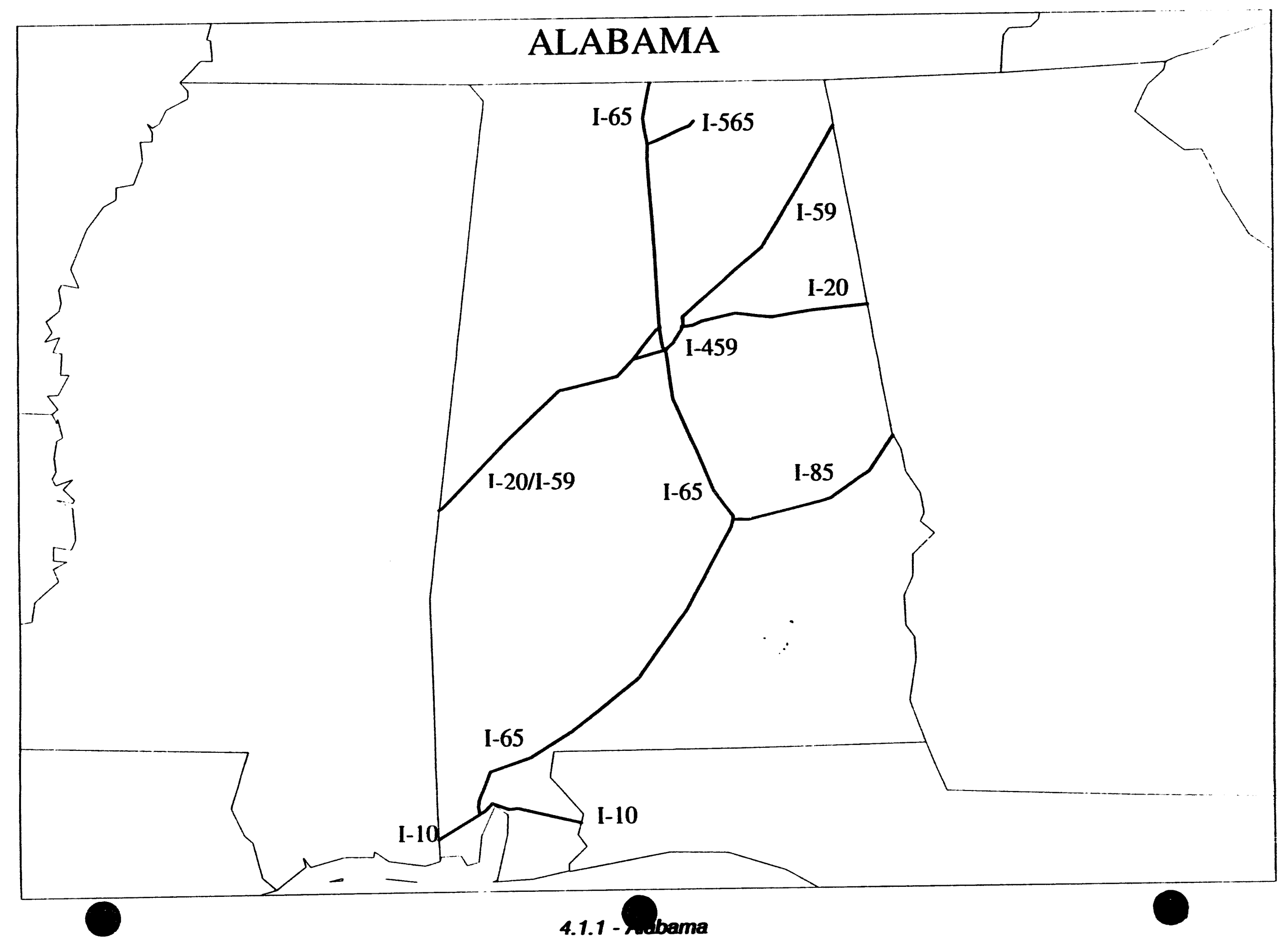




\section{Preferred Routes in Arizona}

1-10 - From the California state line to the junction with I-17 west of Phoenix

- From the junction with I-17 south of Phoenix to the New Mexico state line

I-15 - From the Nevada state line to the Utah state line

1-17 - From the junction with I-10 south of Phoenix to I-40 in Flagstaff

I-19 - From Mexican border to 1-10 in Tucson

I-40 - From California state line to the New Mexico state line

I-8 - From I-10 to the California state line

\section{State Designated Preferred Routes}

Arizona has not designated any additional roads. 


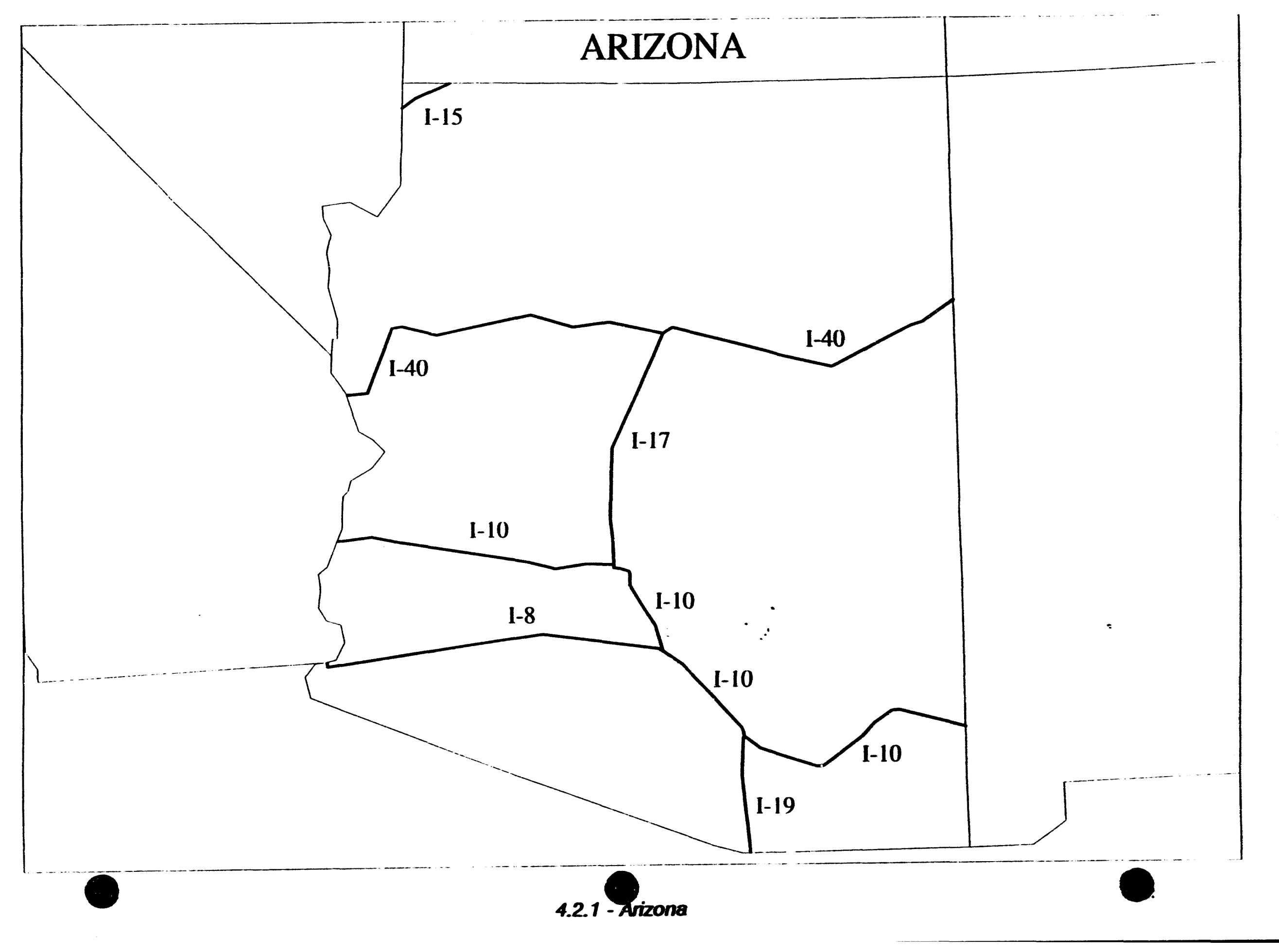




\section{Preferred Routes in Arkansas}

1-30 From the Texas state line to the junction with 1-440 south of Little Rock

- From the junction with 1-30 southwest of Little Rock to the junction with I-40 northwest of North Little Rock

I-40 From Oklahoma state line to the Tennessee state line

I-440 - From the junction with I-30 south of Little Rock to I-40 east of North Little Rock

I-55 - From Tennessee state line to the Missouri state line

\section{State Designated Preferred Routes}

Arkansas has not designated any additional roads. 


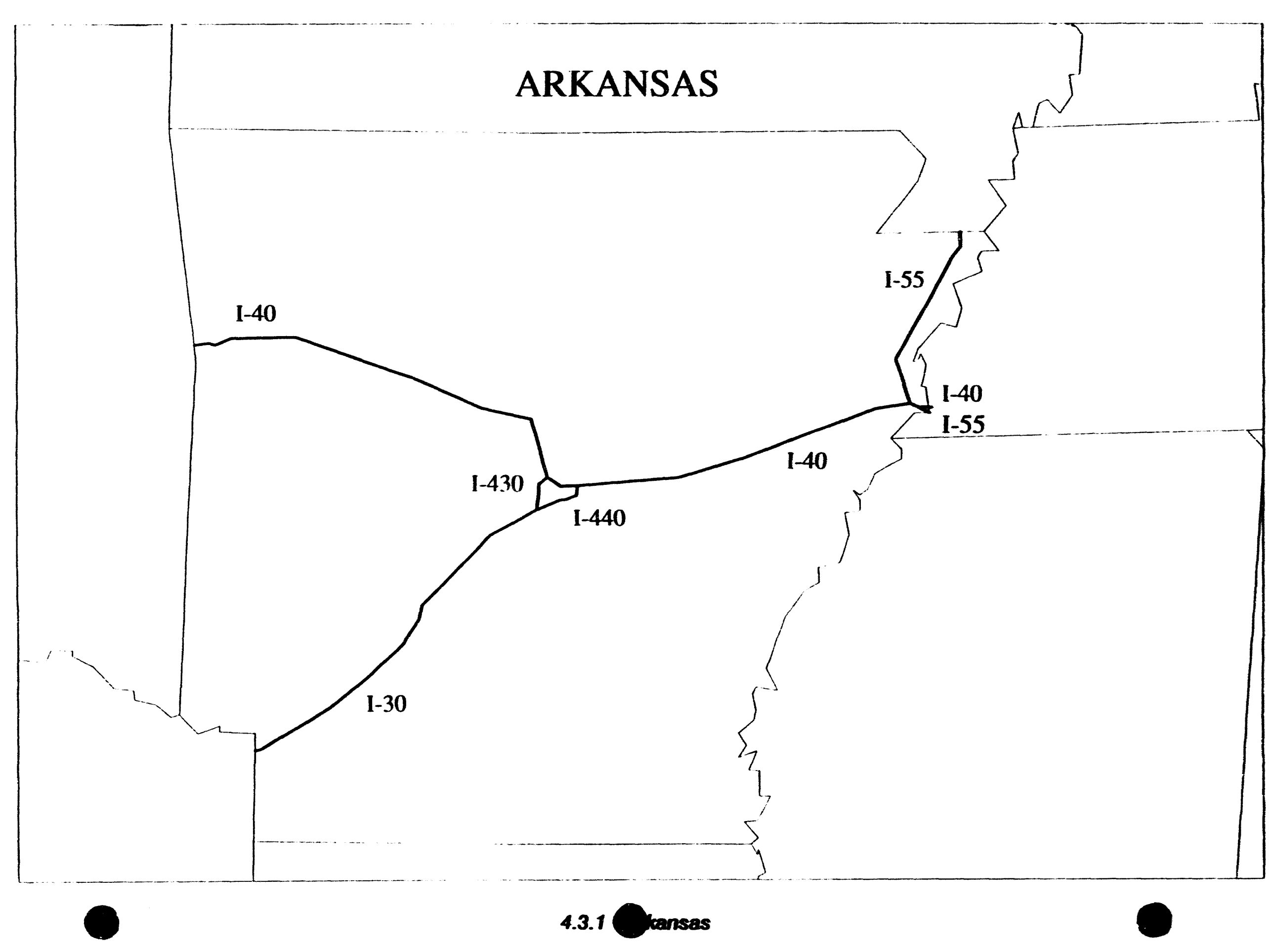




\section{Preferred Routes in California}

1-10

1-15

1-210

I-215

[-280)

1-40

I-405

$1-5$

I-505

I-581)

1-680

1-780

$1-8$

$1-80$

I-805

- From the Arizona state line to junction with 1-605 near Baldwin Park

- From the Nevada state line to 1-8 in San Diego

- From 1-5 east of Tracy to 1-580 west of Tracy

- From I-5 in northwestern Los Angeles to I-10 near Pomona

- From I-15 northwest of San Bernardino to 1-15 northwest of Temecula

- From I-680 in San Jose to San Francisco

- From the Arizona state line to 1-15 near Barstow

- From 1-5 in northwestern Los Angeles to I-5 in Santa Ana

- From the Oregon state line to the junction of 1-405 in northwestern Los Angeles

- From the junction of 1-605 west of Whittier to the junction of I-805 north of San Diego

- From 1-5 northwest of Woodland to 1-80 northeast of Vacaville

- From 1-5 southwest of Vernalis to 1-80 in Oakland

- From I-280 in San Jose to I-80 southwest of Fairfield

- From I-680 in Benicia to 1-80 in Vallejo

- From the Arizona state line to the junction of 1-805 in San Diego

- From the Nevada state line to San Francisco

- From 1-5 north of San Diego to San Ysidro

\section{State Designated Preferred Routes}

California has not designated any additional roads. 


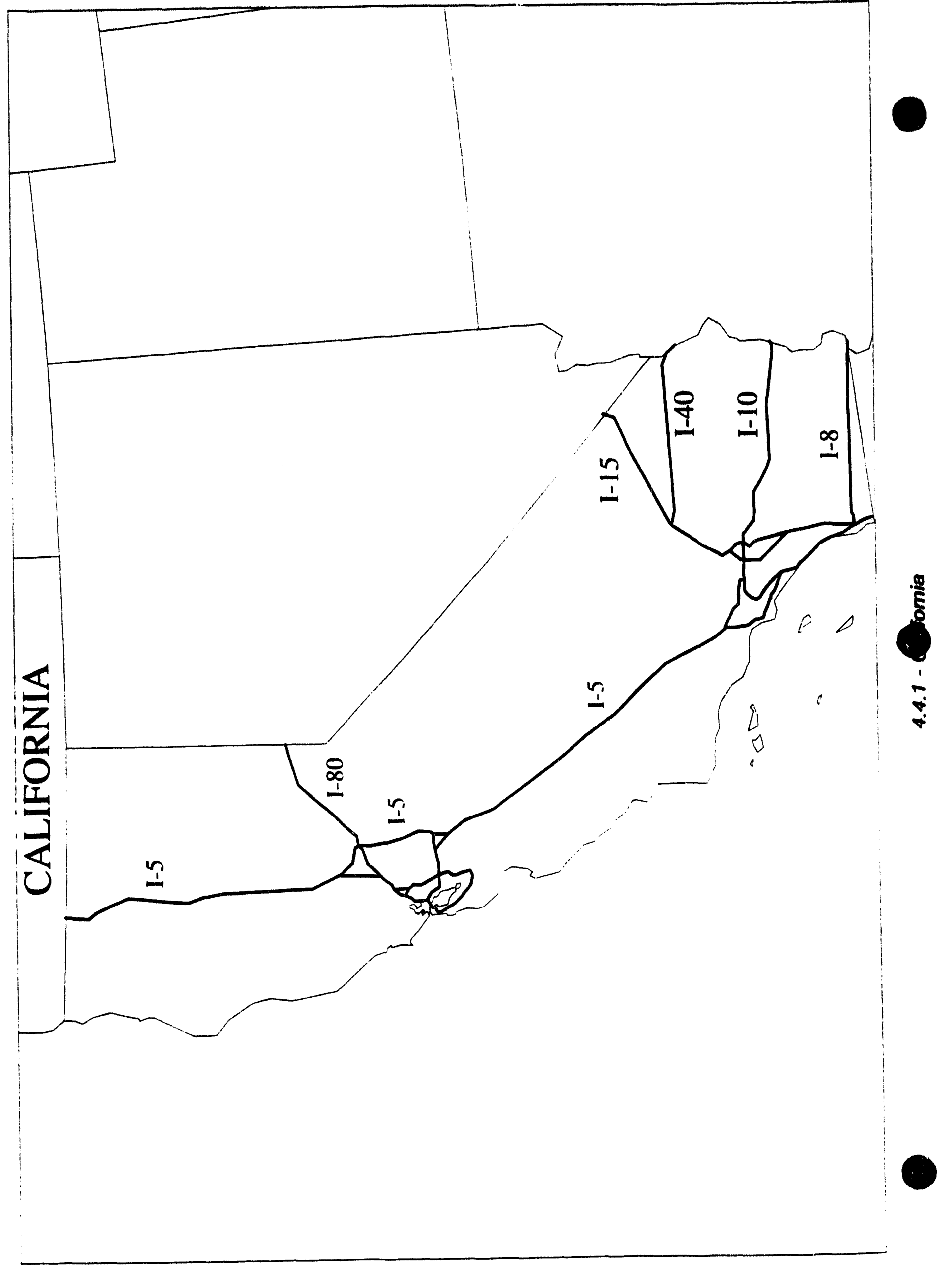




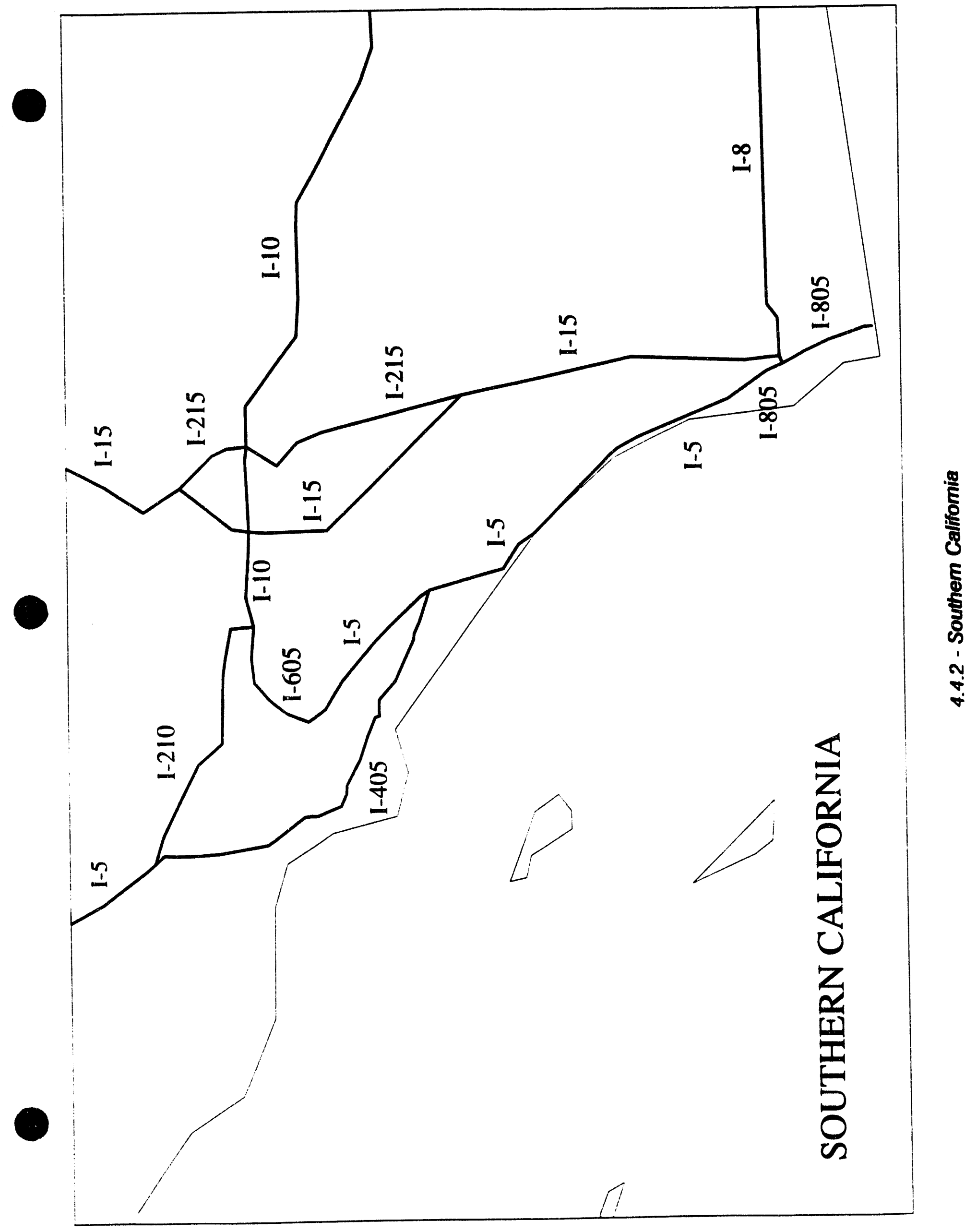




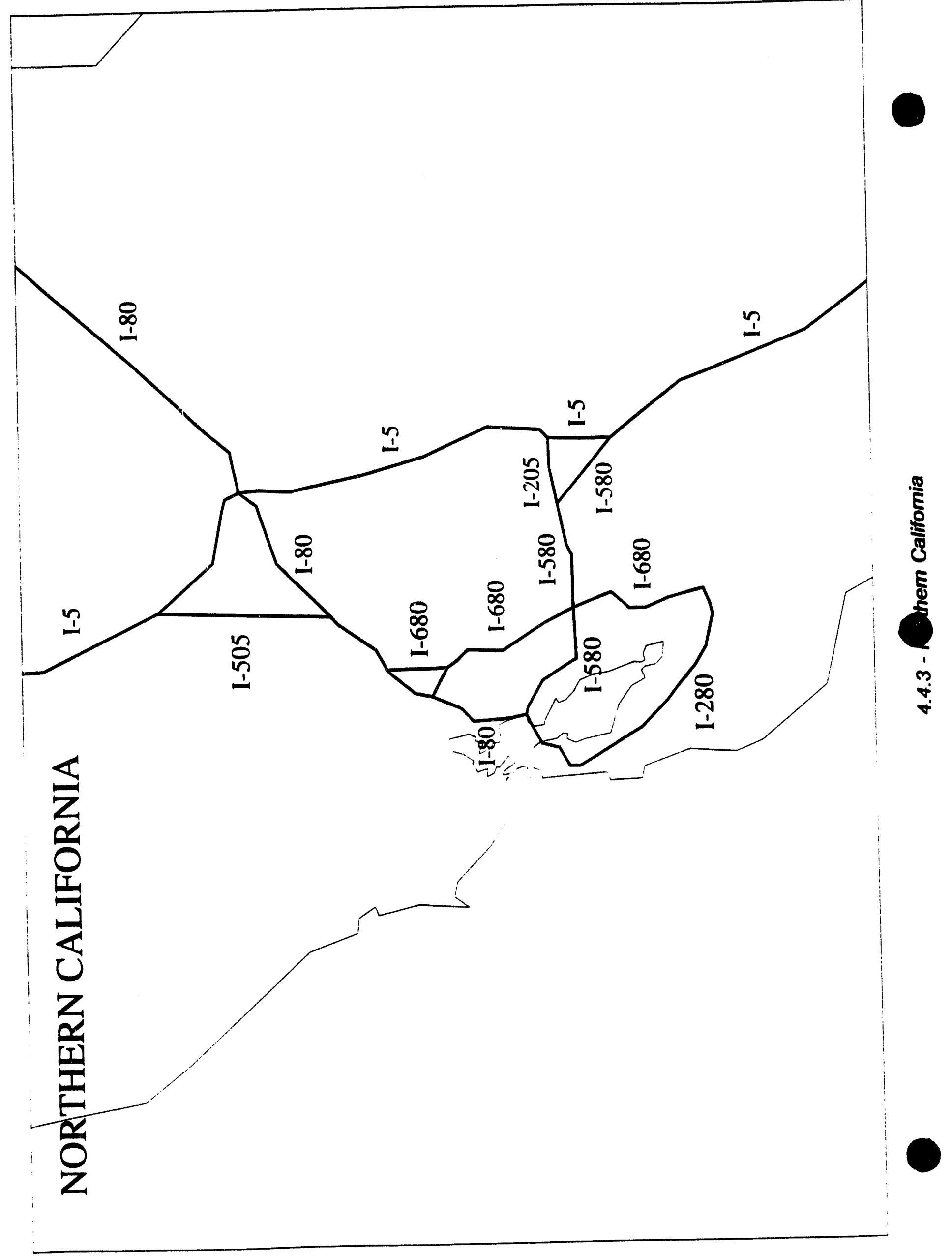




\section{Preferred Routes in Colorado}

1-25 From the New Mexico state line to the Wyoming state line

1-225

- From the junction of 1-25 southeast of Denver to the junction of 1-70 east of Denver

[-70

- From the junction of 1-76 north of Denver to the junction of 1-70 east of Denver

I-76

- From the Kansas state line to the Utah state line

- From the Nebraska state line to I-70 northwest of Denver

\section{State Designated Preferred Routes}

Colorado has designated the following roads, effective March 10, 1989, for access to the Rocky Flat Plant.

U.S. 36 - From the junction of 1-25 north of Denver to the junction of State Route(S.R) 128 near Broomfield

S.R. 128 - From the junction with U.S. 36 near Broomfield to the junction of S.R. 93

S.R. 93 - From the junction with S.R. 128 to the entrance of the Rocky Flats Plant. 


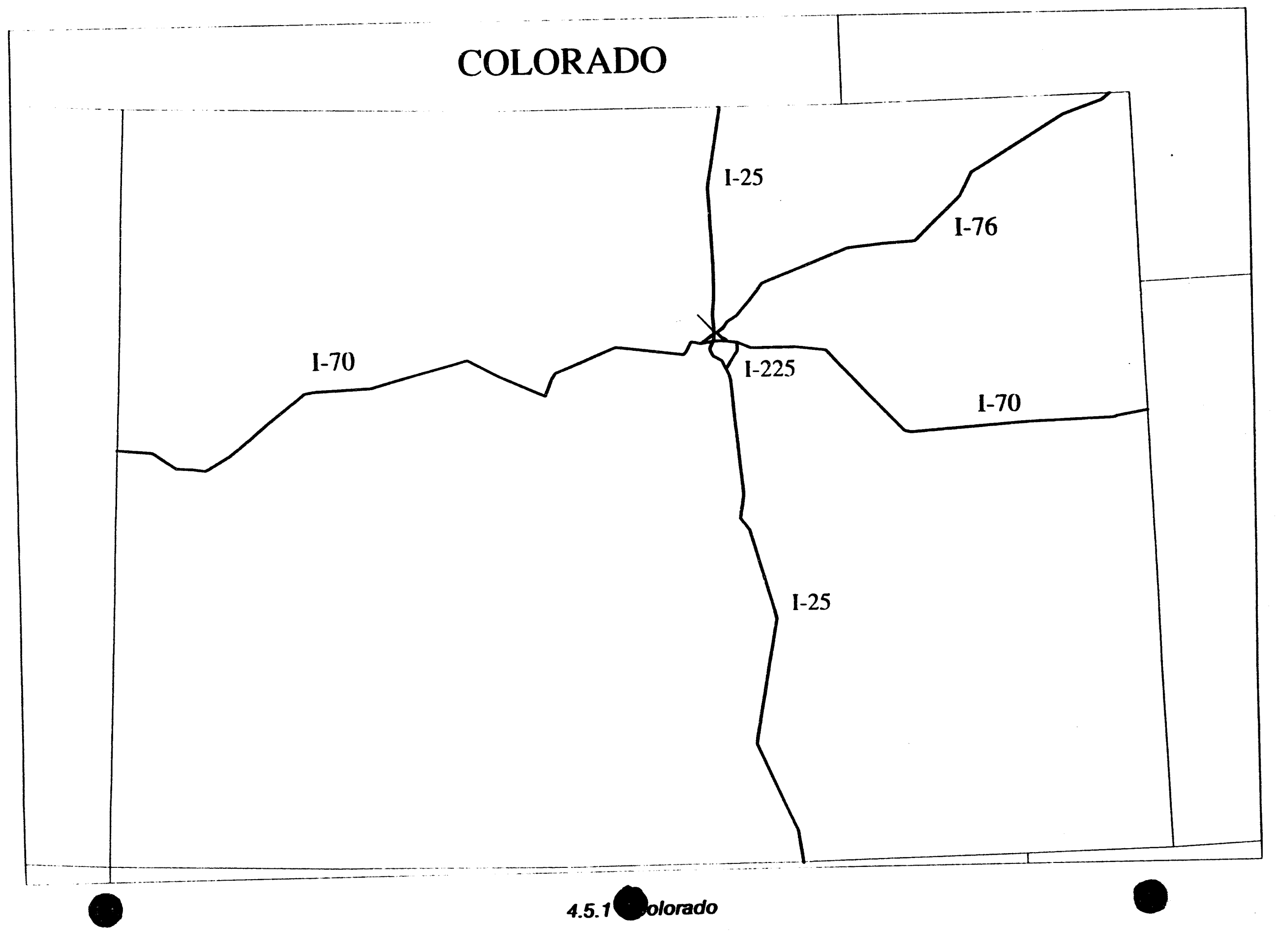




\section{DENVER and vicinity}

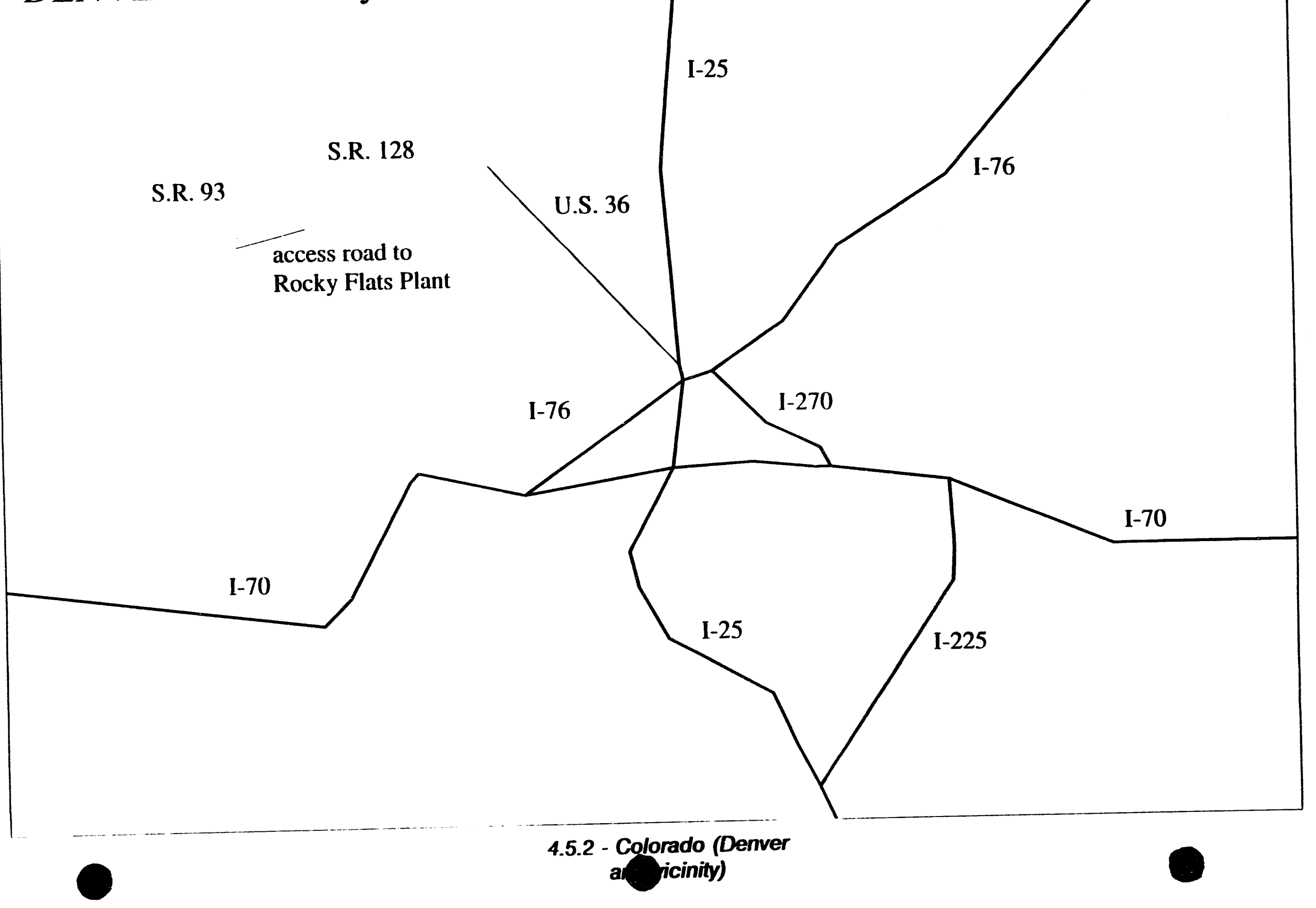




\section{Preferred Routes in Connecticut}

$1-384$

$1-395$

$1-691$

$1-84$

[-9]

I-95
- From the junction with I-84 east of Hartford to the end of I-384 east of Manchester

- From the junction with I-95 west of New London to the Massachusetts state line

- From the New York state line to the New York state line

- From the junction with I-84 east of Waterbury to the junction of I-91 east of Meriden

- From the New York state line to the Massachusetts state line

- From I-95 in New Haven to the Massachusetts state line

- From the New York state line to the Rhode Island state line

\section{State Designated Preferred Routes}

Connecticut has not designated any additional roads. 


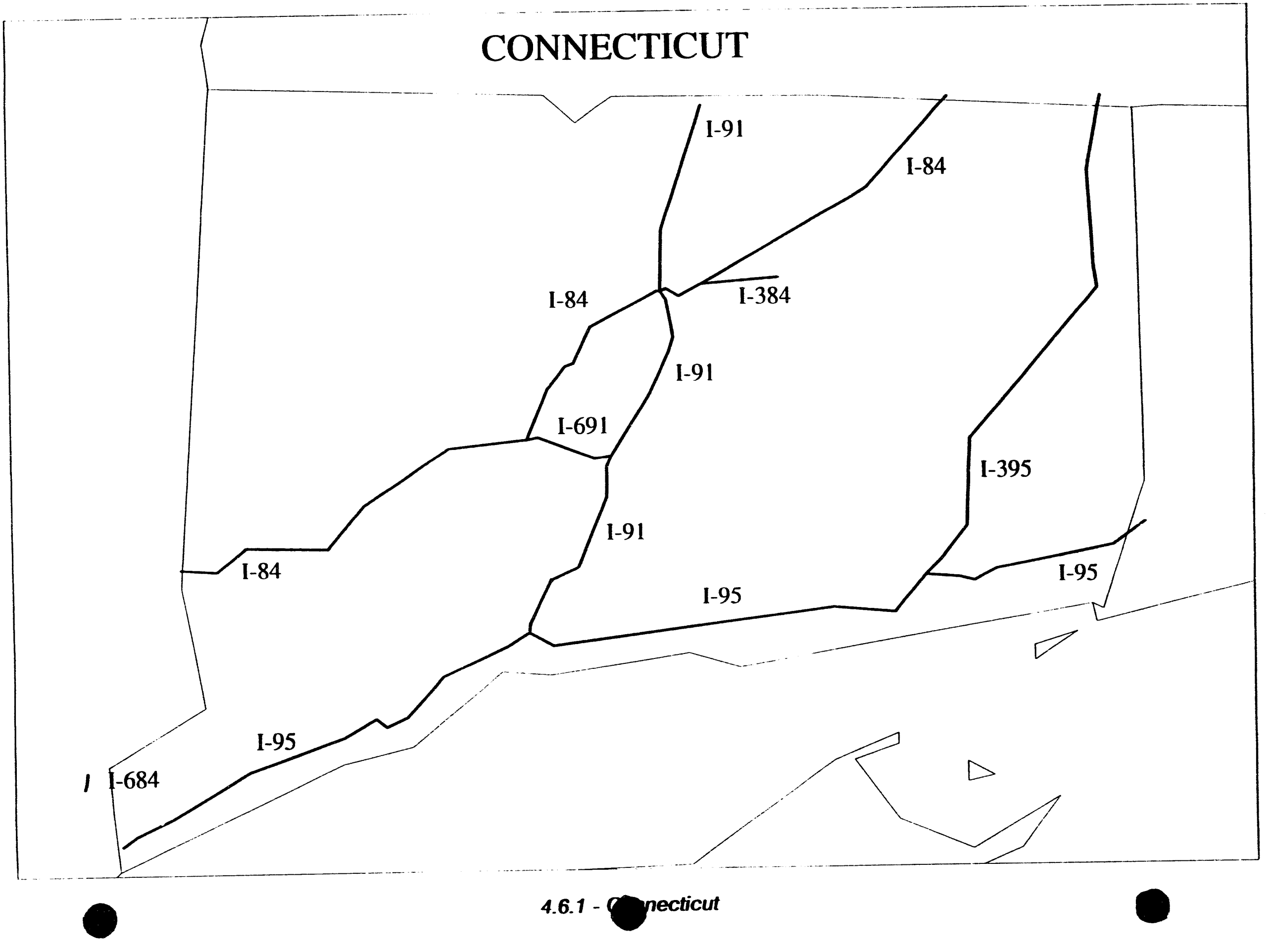




\section{Preferred Routes in Delaware}

1-295

I-495

I-95

- From the junction with I-95 southwest of Wilmington to the New Jersey state line

- From the junction with 1-95 southwest of Wilmington to the junction with 1-95 northeast of Wilmington

- From the Maryland state line to the junction of 1-495 southwest of Wilmington

- From the junction of I-495 northeast of Wilmington to the Pennsylvania state line

\section{State Designated Preferred Routes}

Delaware has not designated any additional roads. 


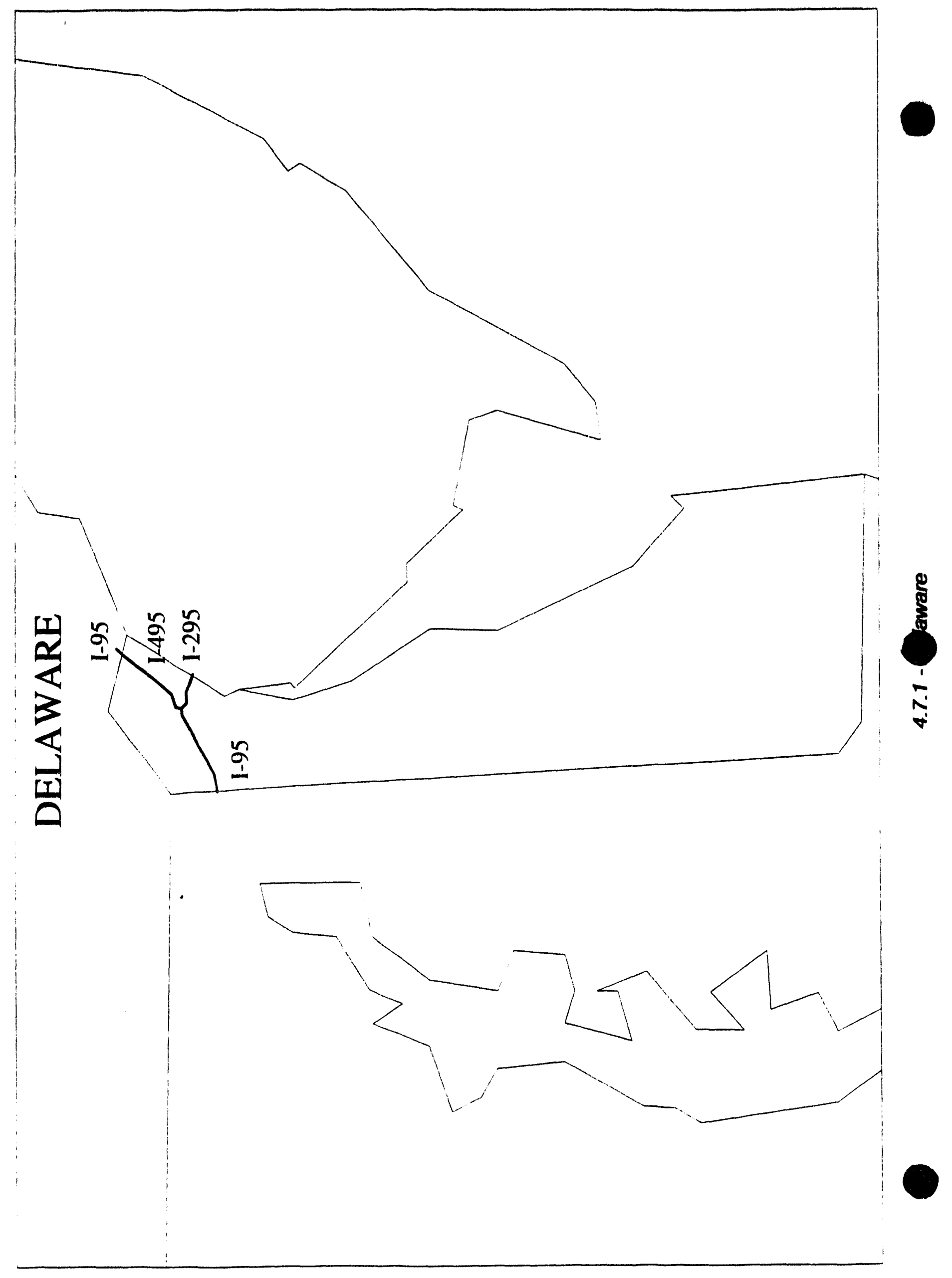




\section{Preferred Routes in the District of Columbia}

By definition, no roads within the District of Columbia are preferred routes. The District of Columbia is within an Interstate System beltway.

\section{State Designated Preferred Routes}

The District of Columbia has not designated any roads. 
0
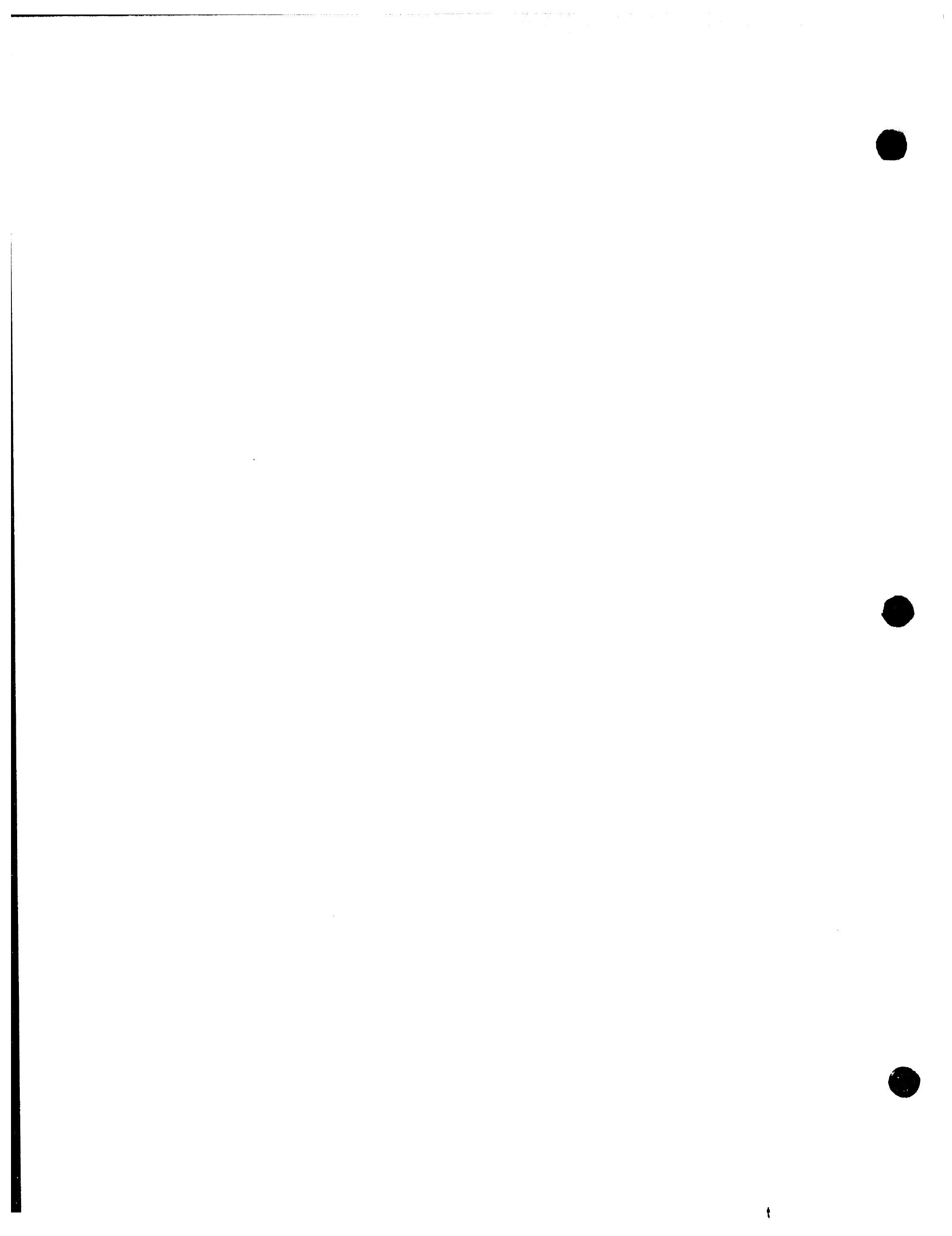

\section{0
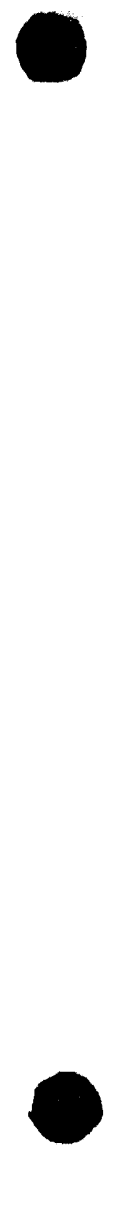 \\ - \\ .}

政

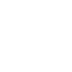




\section{Preferred Routes in Florida}

1-10 - From the Alabama state line to the junction with 1-295 west of Jacksonville

I-295

$1-4$

- From the junction with 1-95 southeast of Jacksonville to the junction with I-95 north of Jacksonville

- From the junction with 1-75 northeast of Tampa to the junction with 1-95 near Daytona Beach

I-75

- From the junction with 1-75 west of Plantation to the junction with 1-95 in Fort Lauderdale

$1-95$

- From Hialeah to the Georgia state line

- From Miami to the junction with 1-295 southeast of Jacksonville

- From the junction with 1-295 north of Jacksonville to the Georgia state line

\section{State Designated Preferred Routes}

Florida has not designated any additional roads. 


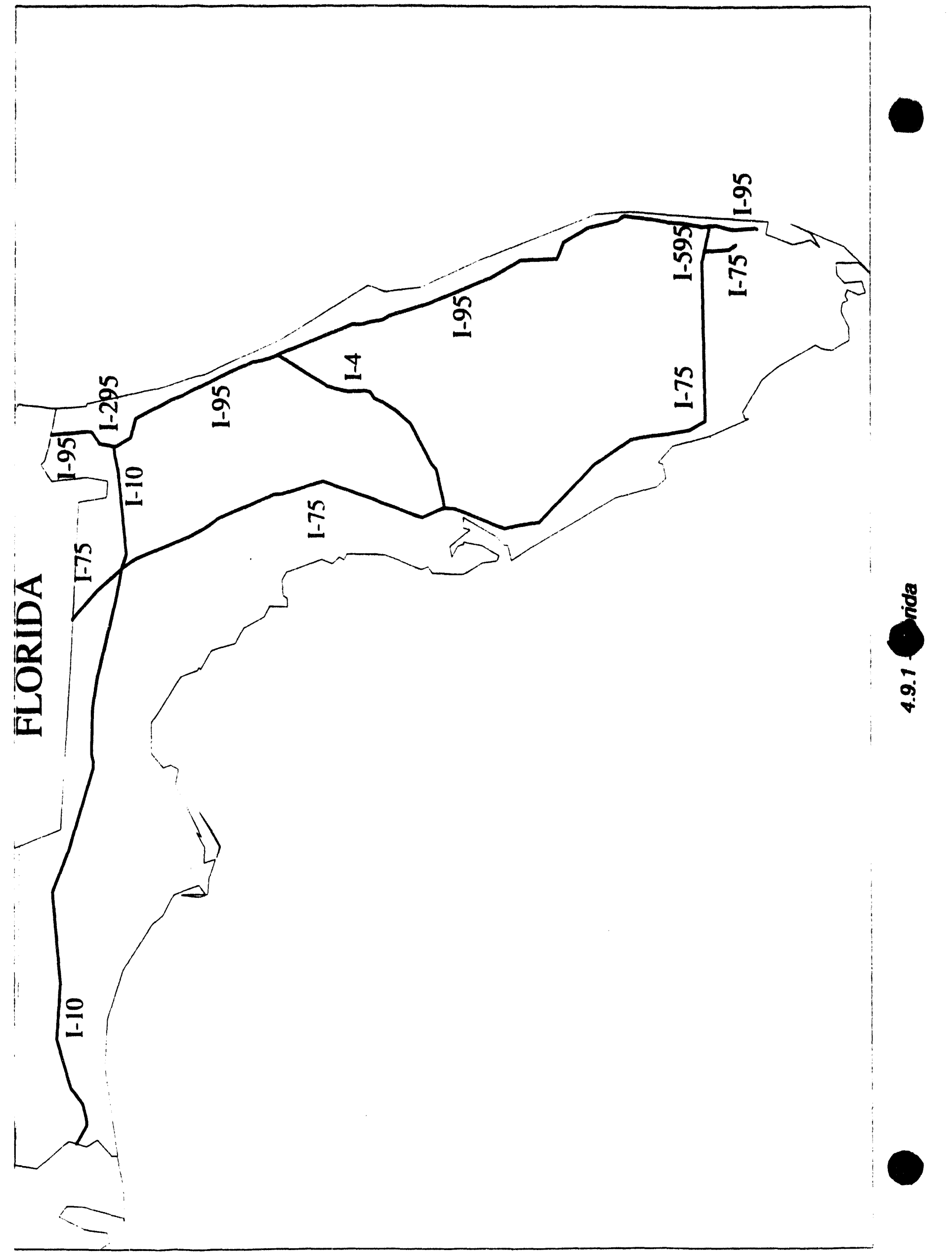




\section{Preferred Routes in Georgia}

I-16

$1-185$

I-20

1-24

1-285

$1-475$

I-520

$1-575$

1-59

I-675

$1-75$

1-85

I-95

$1-985$

- From the junction with 1-75 in Macon to Savannah

- From the Columbus to the junction with 1-85 east of La Grange

- From the Alabama state line to the junction with 1-285 west of Atlanta

- From the junction with 1-285 east of Atlanta to the South Carolina state line

- From the Tennessee state line to the Tennessee state line

- The entire route around Atlanta

- From the junction with I-75 southwest of Macon to the junction with 1-75 northwest of Macon

- From the junction with I-20 west of Augusta to the end of 1-520 suuth of Augusta

- From the junction with 1-75 near Marietta to the end of I-575 near Ball Ground

- From the Alabama state line to the junction with I-24

- From the Alabama stath 1-75 east of Jonesboro to the junction with 1-285 southeast of Atlanta

- From the Florida state line to the junction with I-285 south of Atlanta

- From the junction with 1-285 northwest of Atlanta to ihe Tennessee state line

- From the Alabama state line to the junction with I-285 southwest of Atlanta

- From the junction with I-285 northeast of Atlanta to the South Carolina state line

- From the Florida state line to the South Carolina state line

- From the junction with I-85 south of Buford to Gainesville

\section{State Designated Preferred Routes}

Georgia has not designated any additional roads. 


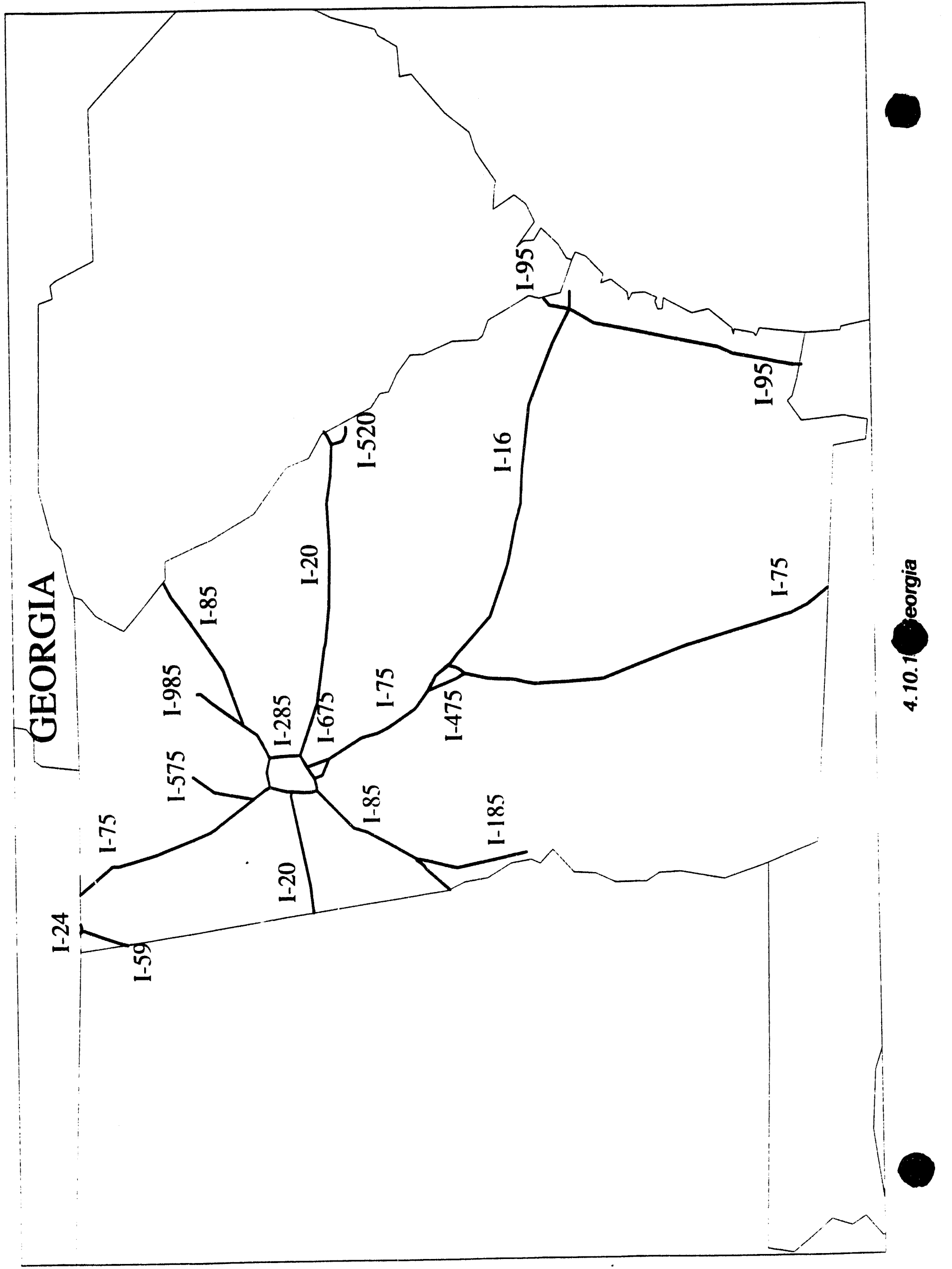



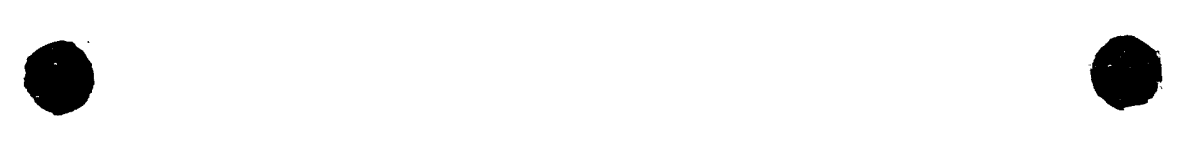

\section{Preferred Routes in Idaho}

I-15 From the Montana state line to the Utah state line

1-84 From the Oregon state line to the Utah state line

I-86 - From I-15 to I-84

I-90 - From the Montana state line to the Washington state line

\section{State Designated Preferred Routes}

Idaho has not designated any additional roads. 


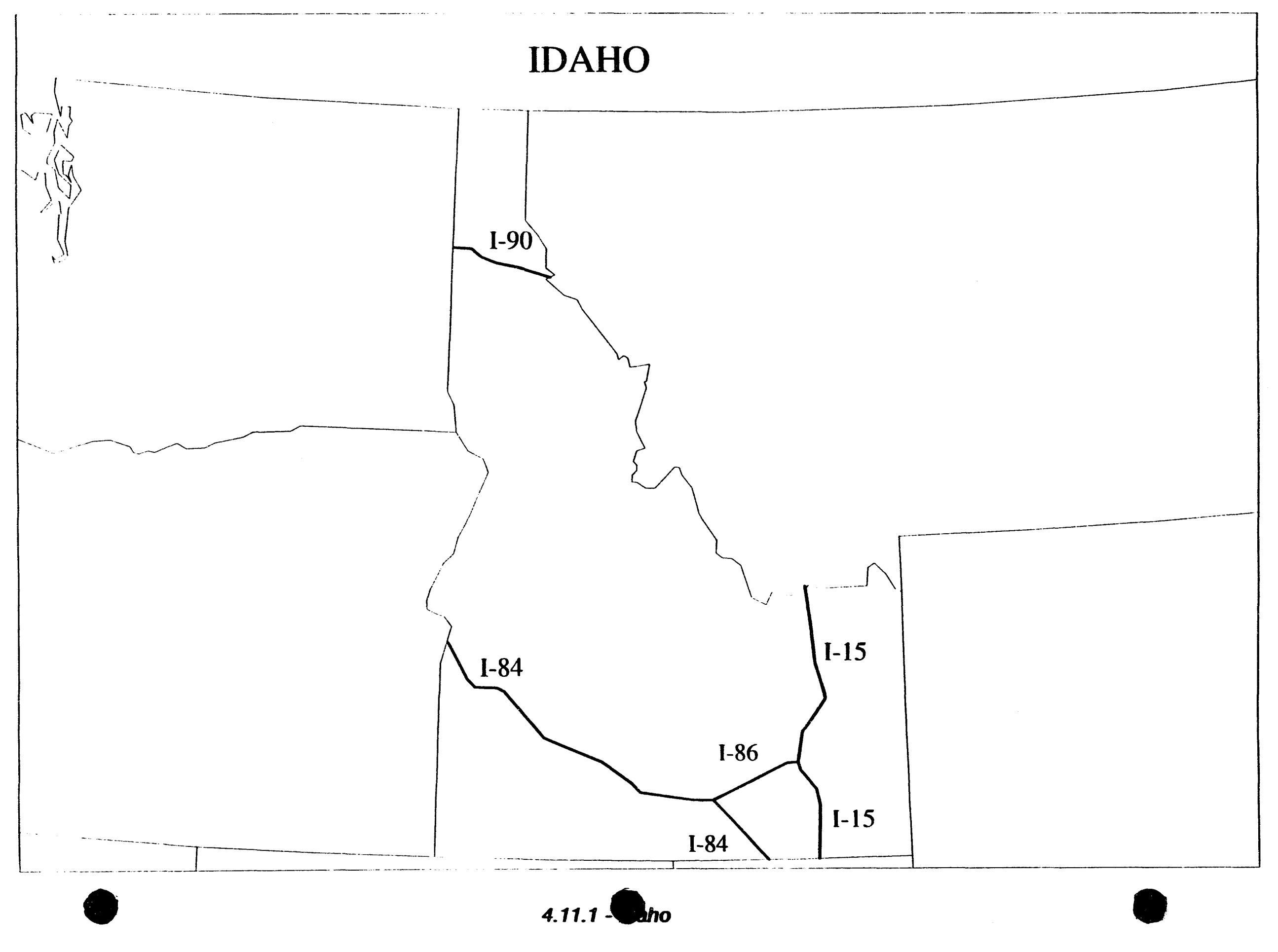




\section{Preferred Routes in Illinois}

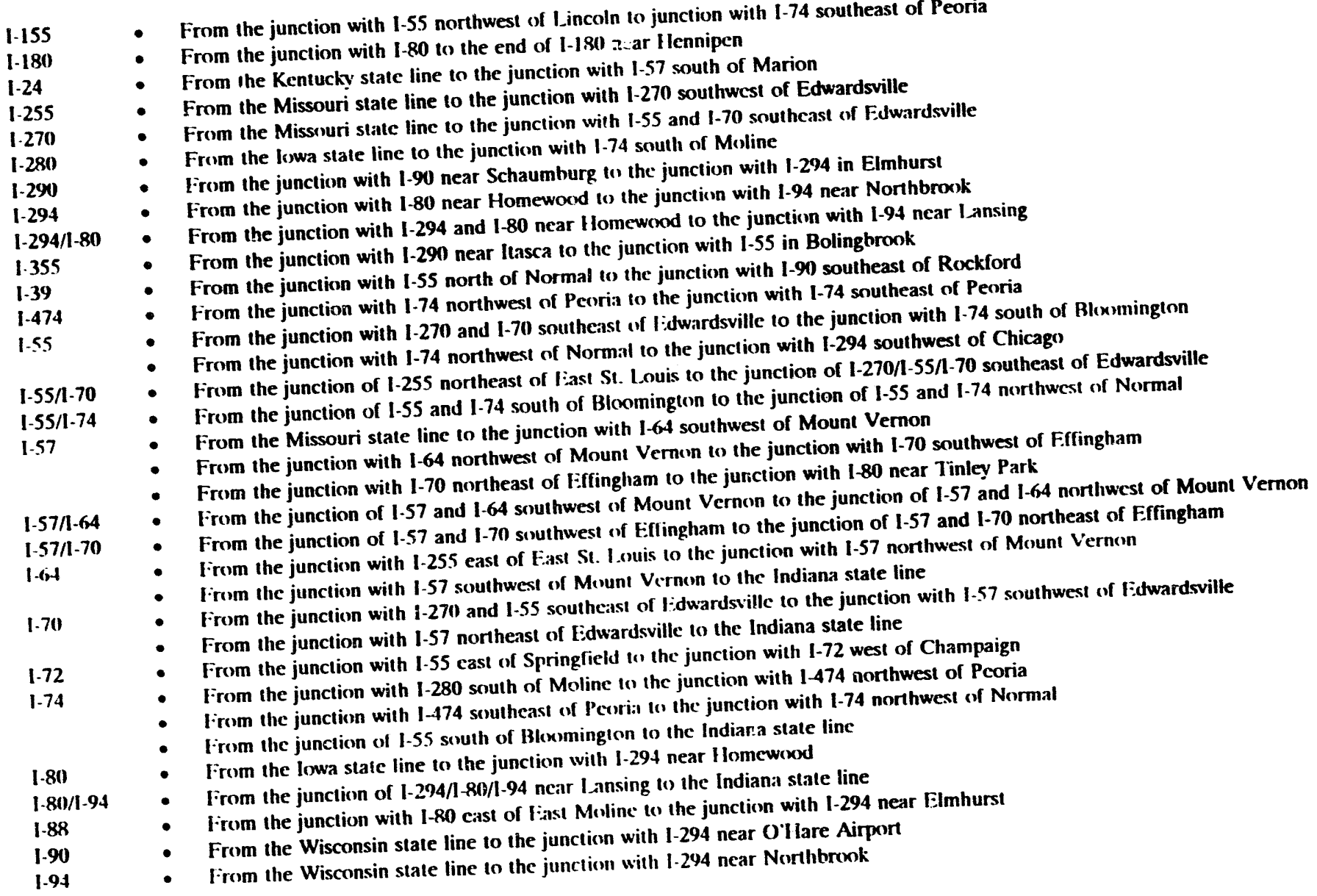

\section{State Designated Preferred Routes}

Illinois has not designated any additional roads.

\subsection{2 - Illinois}




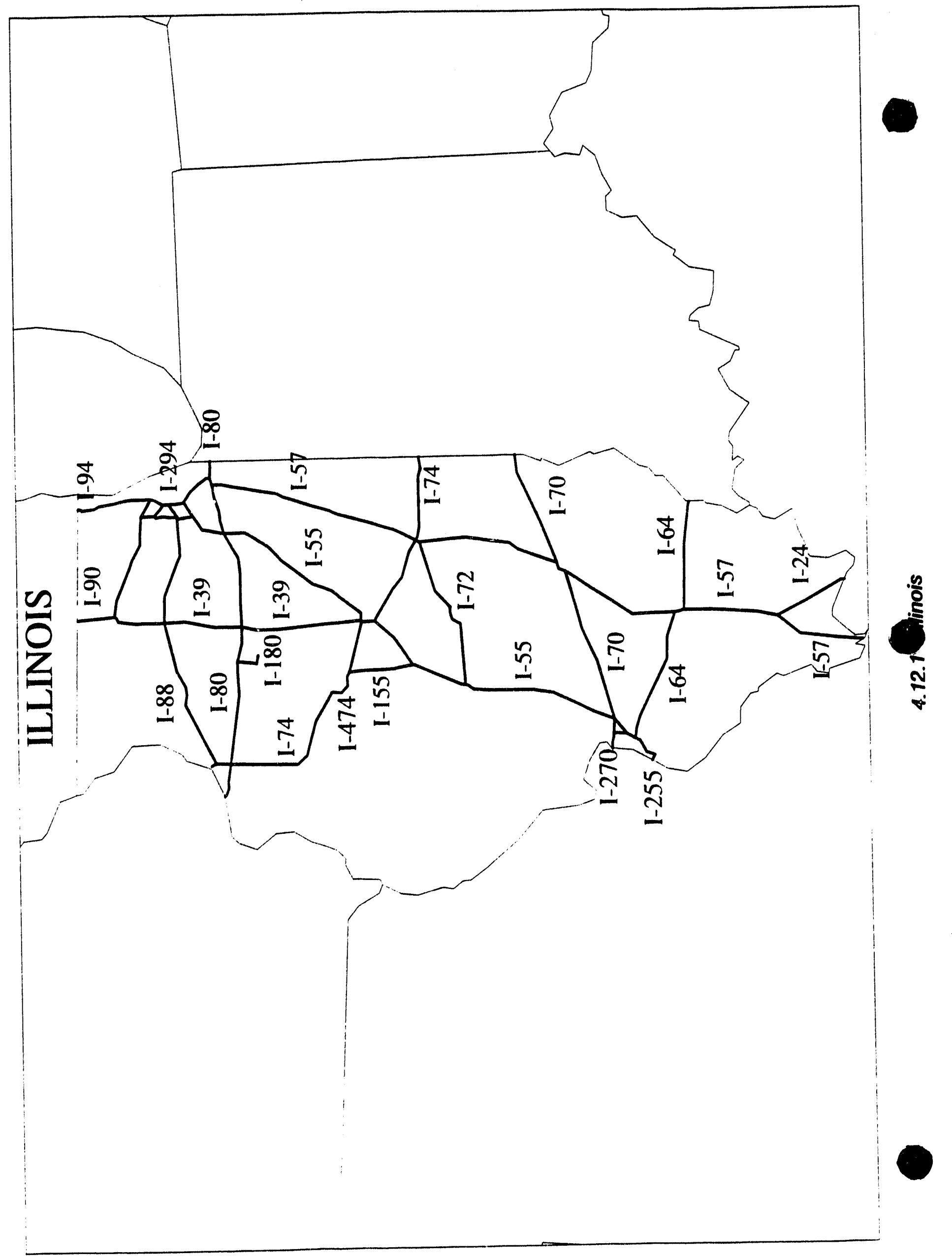




\section{Preferred Routes in Indiana}

I-265

1-275

I-465

1-64

I-70

$1-74$

I-80//-90 - From the junction of I-80/I-90//I-94 near Portage to the Ohio state line

1-80/[-94 - From the Illinois state line to the junction of I-80/1-90//-94 near Portage

- From the junction of $1-80 / 1-90 / 1-94$ near Portage to the Michigan state line

\section{State Designated Preferred Routes}

Indiana has not designated any additional roads. 


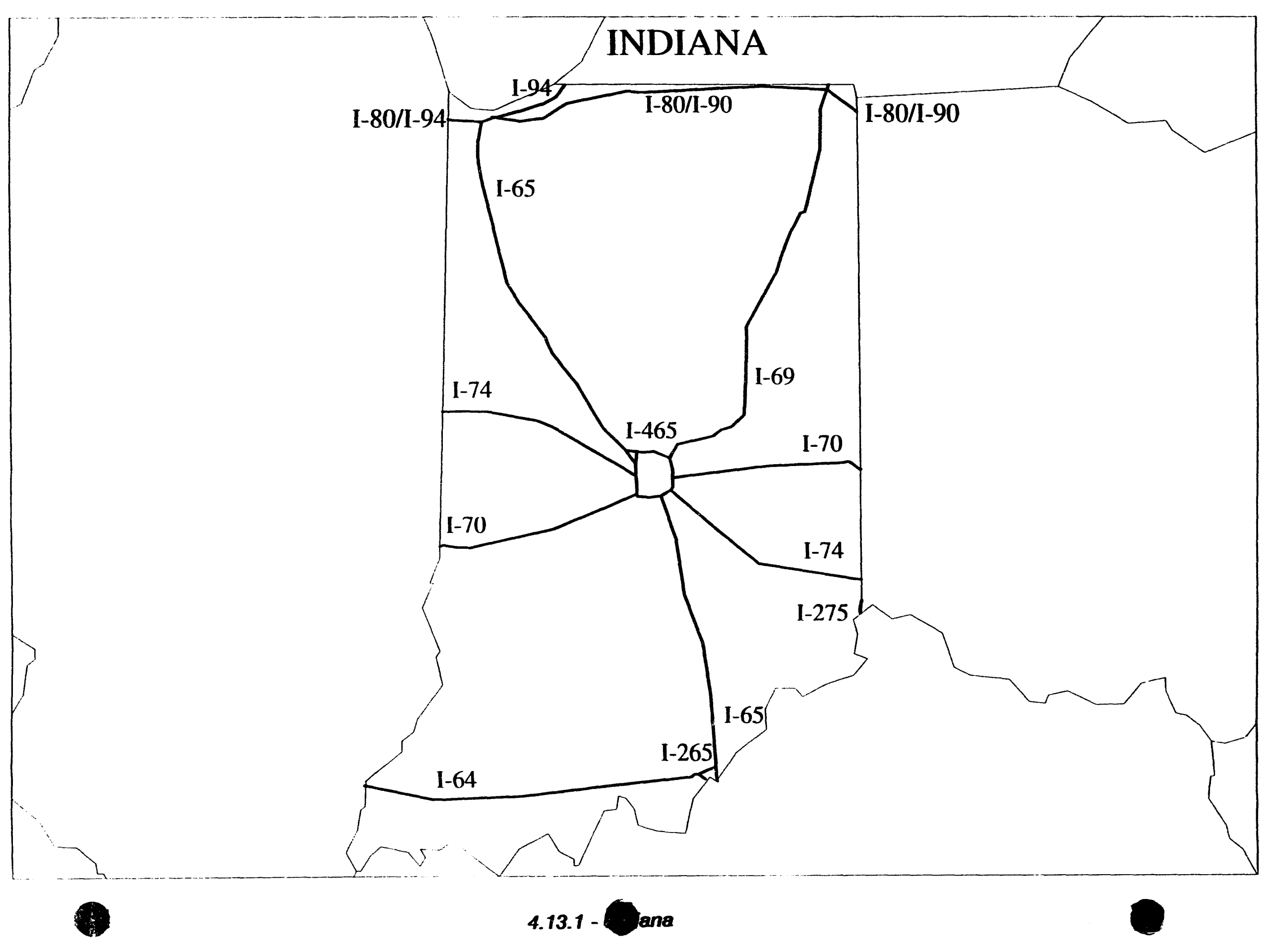




\section{Preferred Routes in lowa}

I-129

$1-280$

1-29 - From the Missouri state line to the junction with 1-80 southeast of Council Bluffs

- From the junction with 1-680 south of Missouri Valley to the South Dakota state line

I-29/I-680

- From the junction of these roads north of Council Bluffs to south of Missouri Valley

$1-35$

- From the Missouri state line to the junction with 1-80 west of Des Moines

- From the junction with 1-80 north of Des Moines to the Minnesota state line

I-35/I-80 - From the junction of these roads north of Des Moines to west of Des Moines

- From the junction of I-80 northwest of lowa City to Waterloo

1-680

- From the Nebraska state line to the junction with 1-29 north of Council Bluffs

- From the junction with 1-29 south of Missouri Valley to the junction with I-80 northeast of Council Bluffs

I-80

- From the junction with I-29 southeast of Council Bluffs to the junction with I-35 west of Des Moines

- From the junction with I-35 north of Des Moines to the junction with I-280 northwest of Davenport

\section{State Designated Preferred Routes}

Iowa has designated, as of Septemher 1, 1988, that the following routes be used:

I-280 in lieu of 1-80 through the Quad Cities areas

1-35/1-80 in lieu of 1-235 through Des Moines

$\mathrm{I}-80$ and $\mathrm{I}-680$ in lieu of $\mathrm{I}-29$ through the Council Bluffs area 


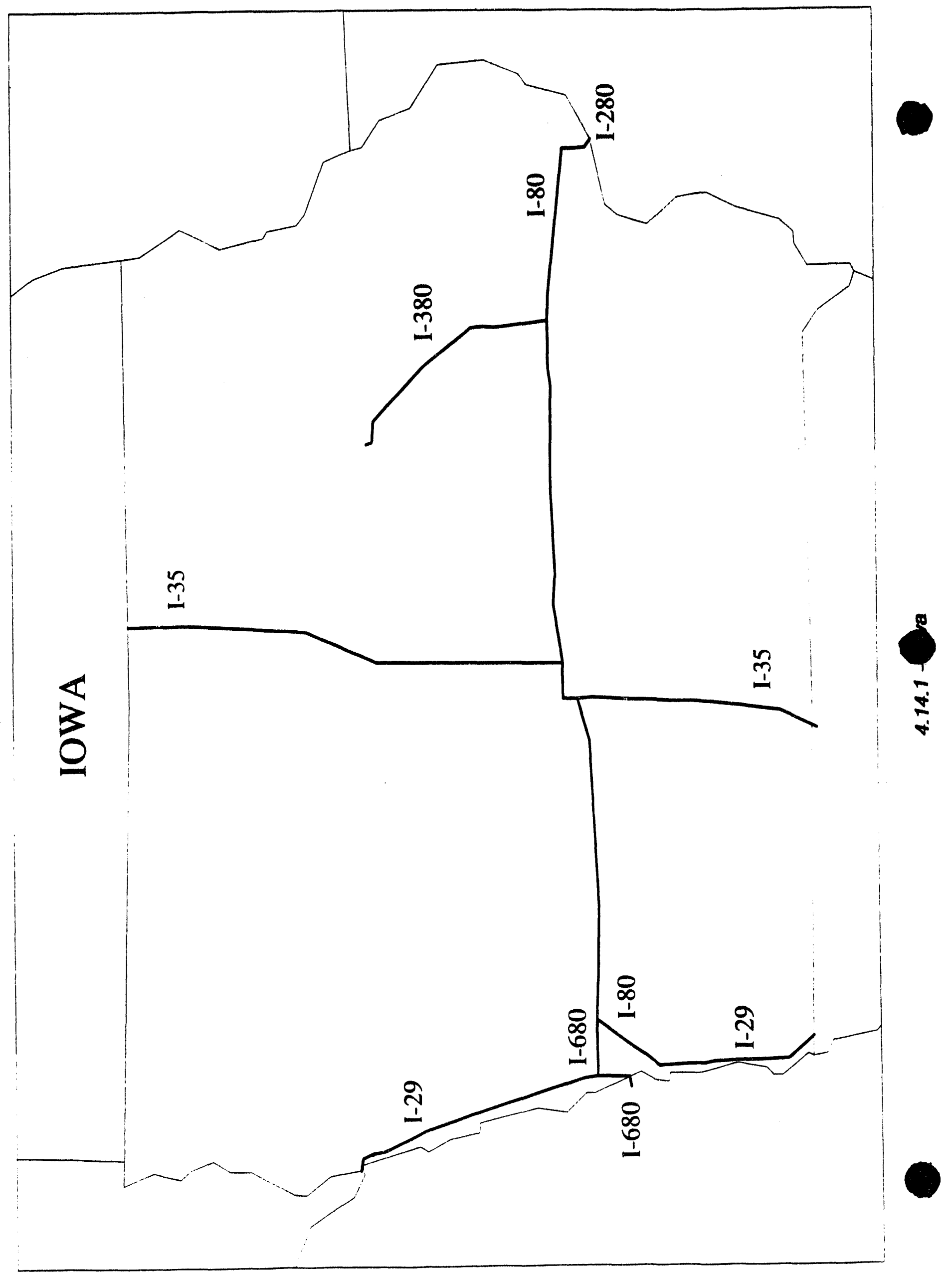




\section{Preferred Routes in Kansas}

I-135 - From the junction with I-235 north of Wichita to the junction with I-70 near Salina

- From the junction with 1-235 north of Wichita to the junction with I-35 south of Wichita

- From the junction with I-35 near Emporia to the junction with I-470 south of Topeka

- From the Oklahoma state line to the junction with I-435 near Overland Park

- From the Missouri state line northwest of Kansas City to the Missouri state line south of Kansas City

- From the junction with I-70 west of Topeka to the junction with 1-70 east of Topeka

$1-70$

- From the Colorado state line to the junction with I-470 west of Topeka

- From the junction with I-470 east of Topeka to the junction with I-435 west of Kansas City

\section{State Designated Preferred Routes}

Kansas has not designated any additional roads. 


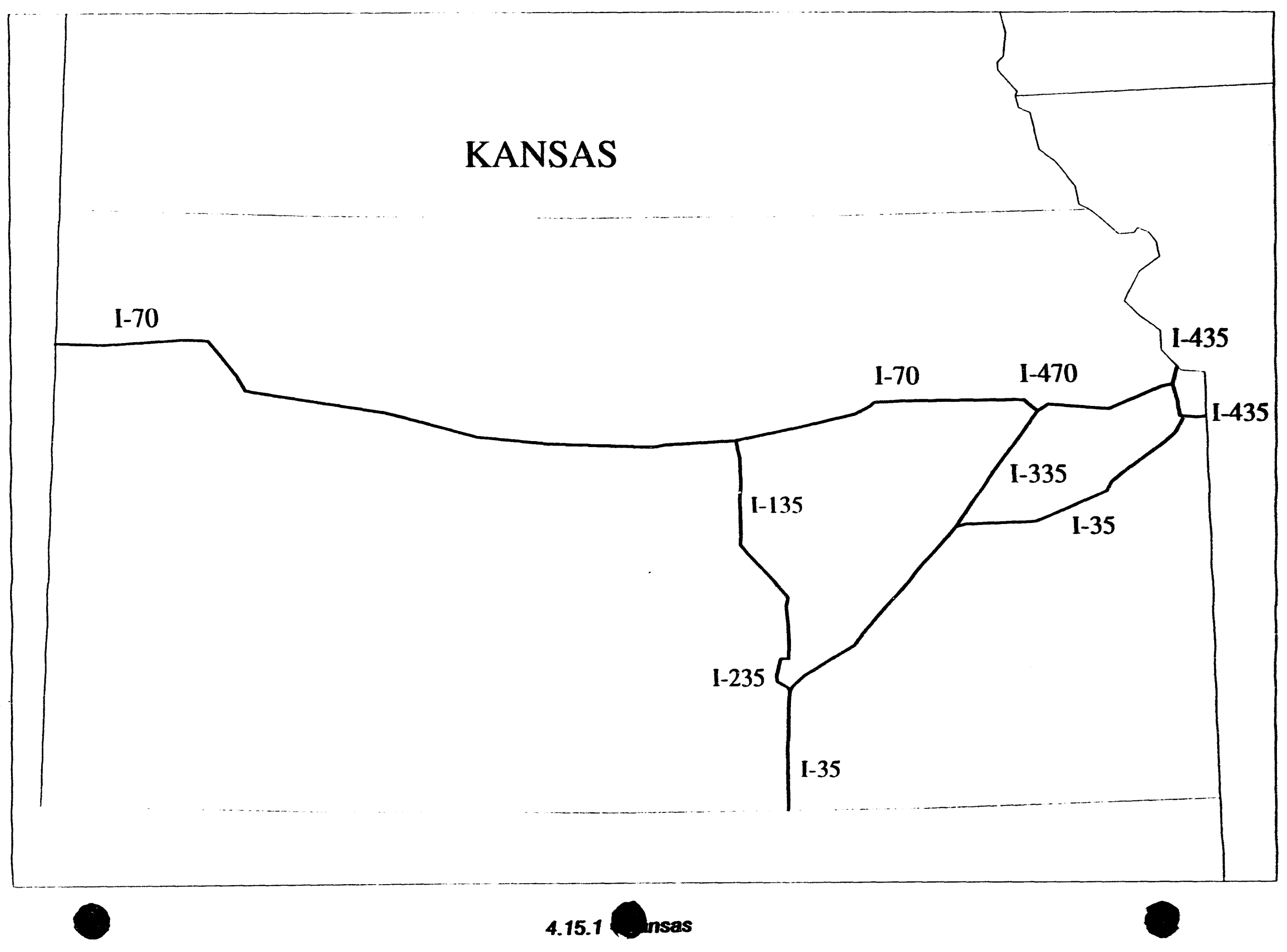




\section{Preferred Routes in Kentucky}

1-24 From the Tennessee state line to the Illinois state line

1-265 - From the junction with I-265 south of Louisville to the junction with I-71 northeast of Louisville

1-275 - From the Ohio state line to the Indiana state line

1-64 From the Indiana state line to the junction with 1-75 north of Lexington

- From the junction with I-75 east of Lexington to the West Virginia state line

I-64/1-75 - From the junction of 1-64 and I-75 north of Lexington to the junction of 1-64 and 1-75 east of Lexington

I-65 - From the Tennessee state line to the Indiana state line

1-71 From the junction with 1-64 in Louisville to the junction with I-71 near Walton

1-71/1-75 - From the junction of I-71 and 1-75 near Walton to the junction with 1-275 near Erlanger

1-75 From the Tennessee state line to the junction with 1-64 east of Lexington

- From the junction with I-64 north of Lexington to the junction with I-71 near Walton

\section{State Designated Preferred Routes}

Kentucky has designated, on November 2.1988 , the above listed highways as the preferred routes in their state. 


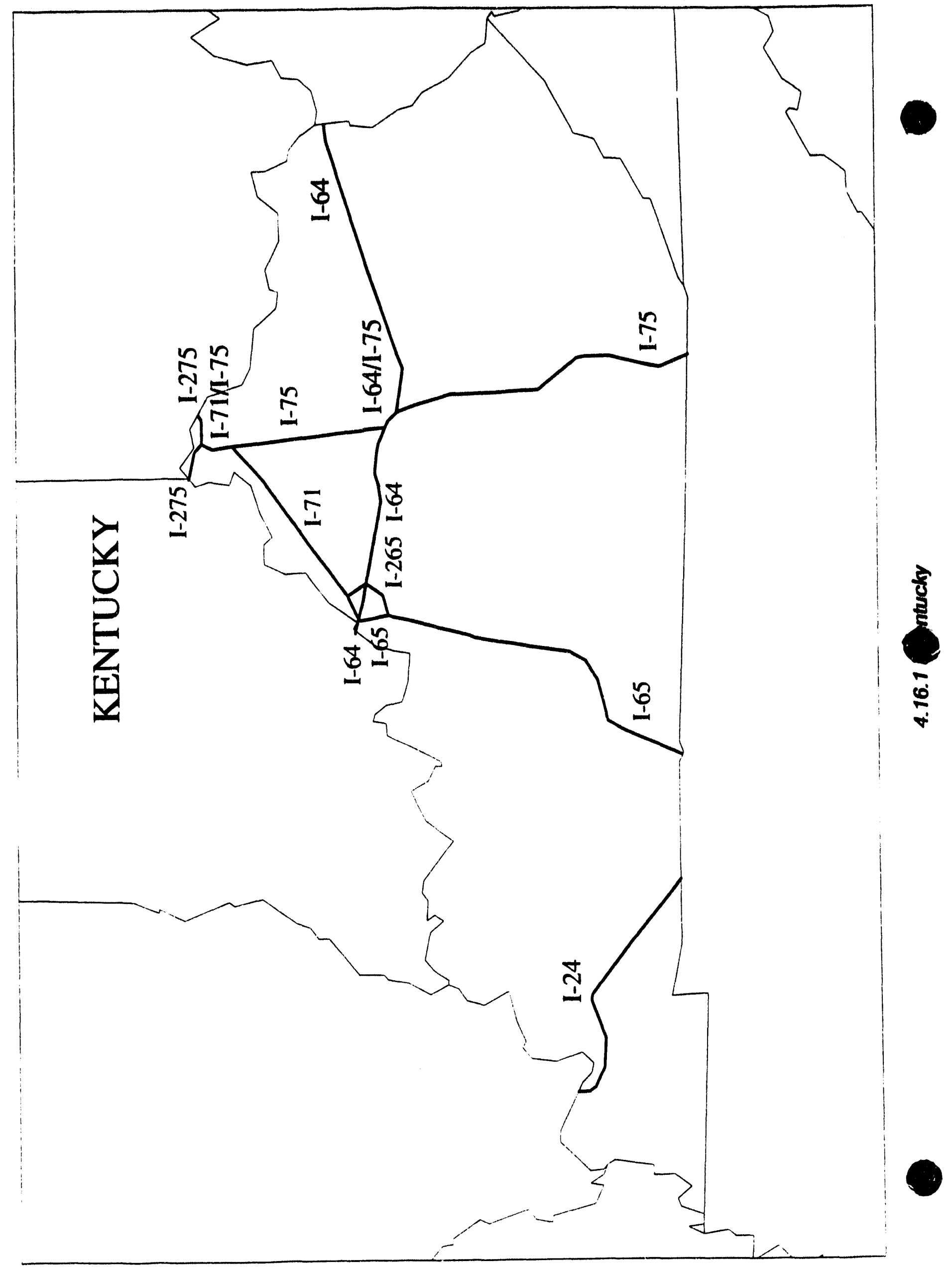




\section{Preferred Routes in Louisiana}

1-10 - From the Texas state line to the junction with 1-610 northwest of downtown New Orleans

- From the junction with I-610 northeast of downtown New Orleans to the Mississippi state line

$1-110$

- From the junction with 1-10 in Baton Rouge to the end of 1-110

$1-12$

- From the junction with I-10 in Baton Rouge to the junction with 1-10 and I-59 near Slidell

$1-20$

- From the Texas state line to the junction with 1-220 west of Shreveport

- From the junction with 1-220 east of Bossier City to the Mississippi state line

- From the junction with 1-20 west of Shreveport to the junction with 1-20 east of Bossier City

- From the junction with 1-10 west of New Orleans to the end of 1-310

$1-49$

- From the junction with I-10 near Lafayette to Shreveport (parts of this road are not complzted yet)

$1-510$

- From the junction with 1-10 east of New Orleans to the end of 1-510

$1-55$

- From the Mississippi state line to the junction with I-10 near La Place

$1-59$

- From the Mississippi state line to the junction with 1-10 and 1-12 near Slidell

I-610

- From the junction with 1-10 northwest of downtown New Orleans to the junction with 1-10 northeast of downtown New Orleans

\section{State Designated Preferred Routes}

Louisiana has not designated any additional roads. 


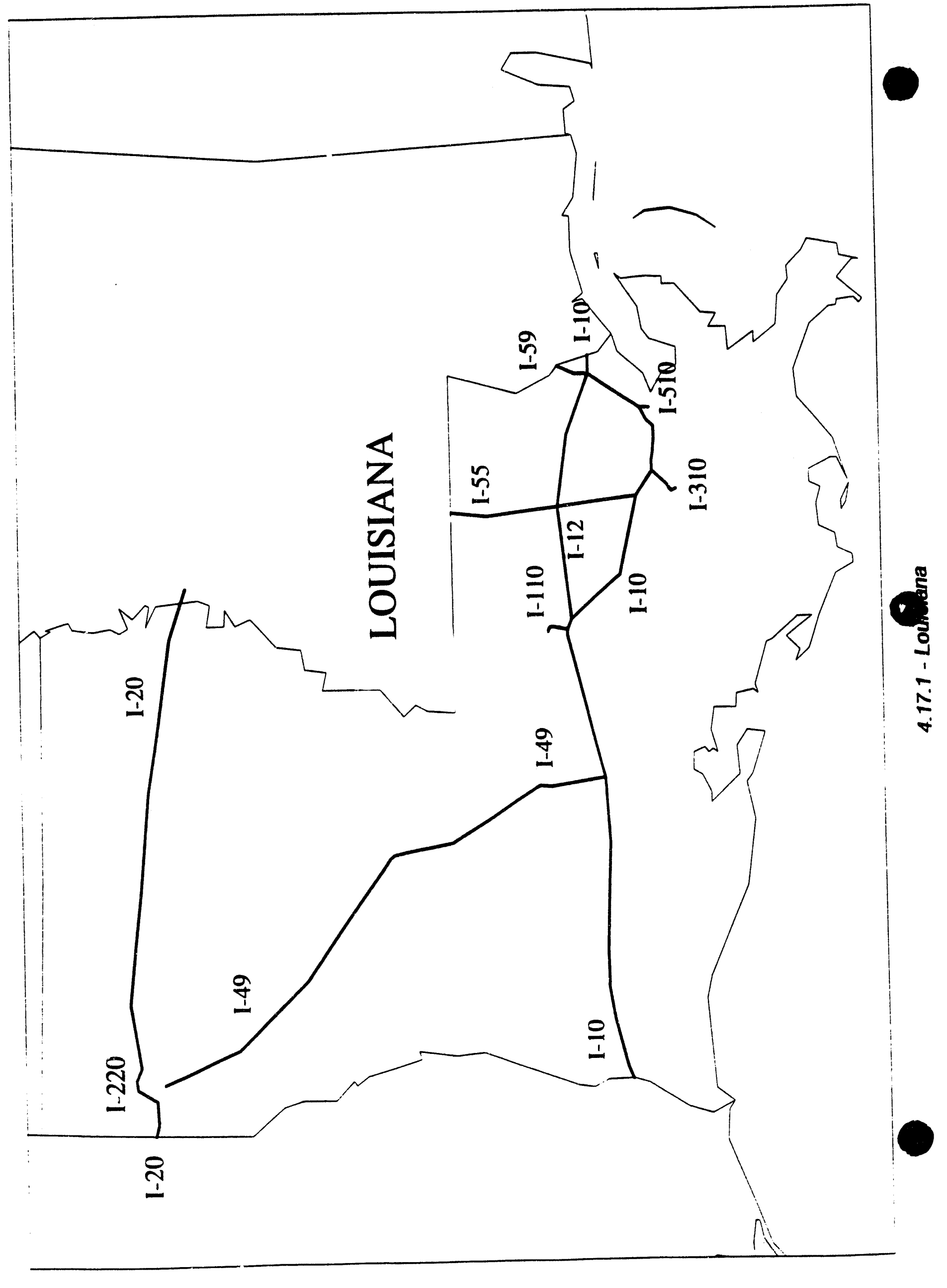




\section{Preferred Routes in Maine}

I-495

I-95

- From the junction with 1-95 north of Portland to the junction with 1-95 south of Augusta

- From the New Hampshire state line to the Canadian border

\section{State Designated Preferred Routes}

Maine has not designated any additional roads. 


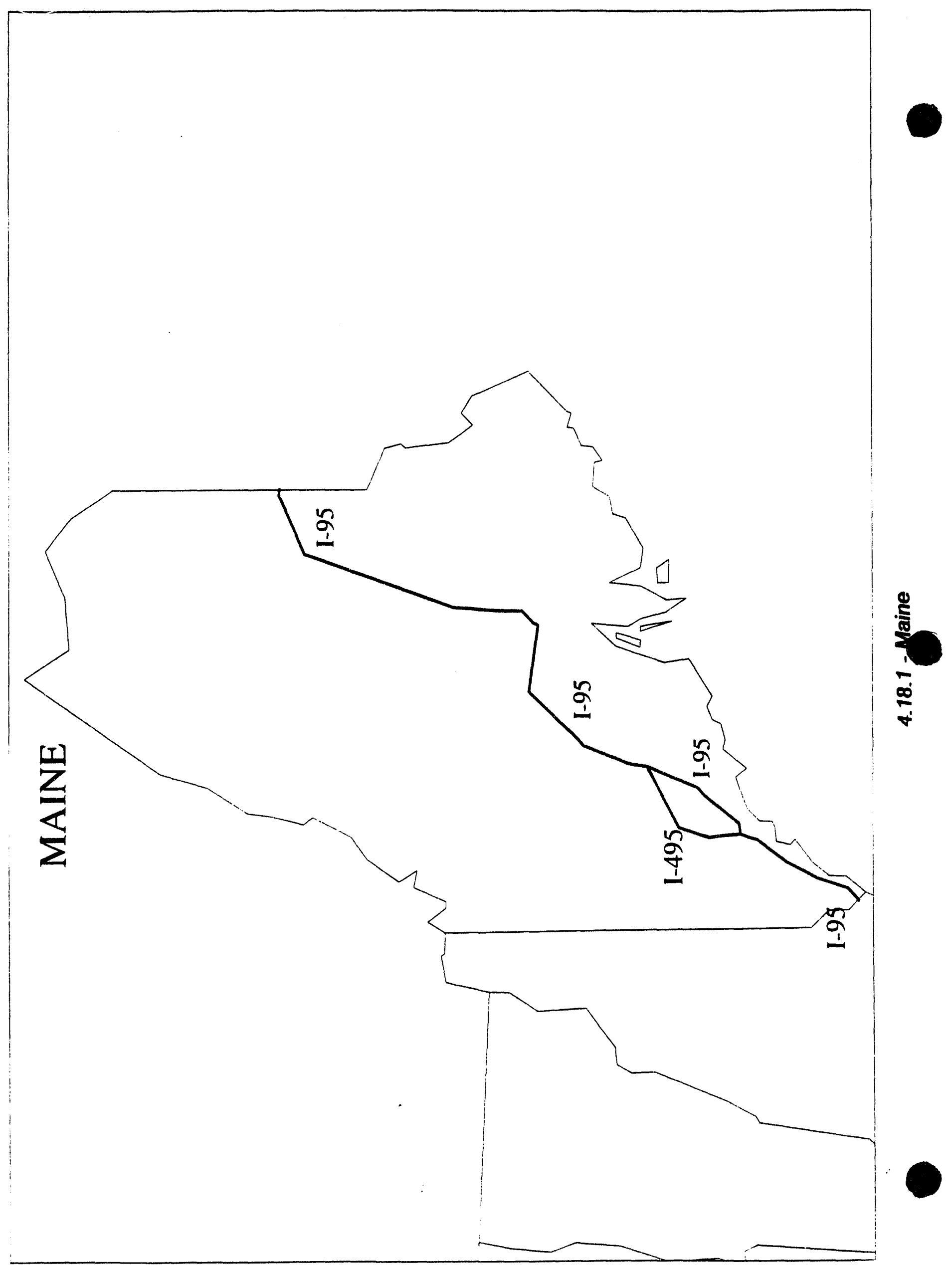




\section{Preferred Routes in Maryland}

I-270

- From the junction with I-495 north of Bethesda to the junction with I-70 in Frederick

I-270Spur

- From the junction with I-494 northwest of Bethesda to the junction with I-270 south of Rockville

$1-495$

- From the Virginia state line to the junction with I-95 southwest of Beltsville

I-495/I-95

- From the Virginia state line to the junction with 1-95 southwest of Beltsville

I-68

- From the West Virginia state line to the junction with 1-70 near Hancock

- The entire route around Baltimore

I-70

- From the Pennsylvania state line to the junction with I-695 west of Baitimore

- From the West Virginia state line to the Pennsylvania state line

- From the junction with I-695 north of Baltimore to the Pennsylvania state line

- From the junction with I-495 southwest of Beltsville to the junction with I-695 southwest of Baltimore

- From the junction with I-695 northeast of Baltimore to the Delaware state line

- From the junction with I-695 south of Baltimore to the end of 1-97 near Annapolis

\section{State Designated Preferred Routes}

Maryland has not designated any additional roads. 


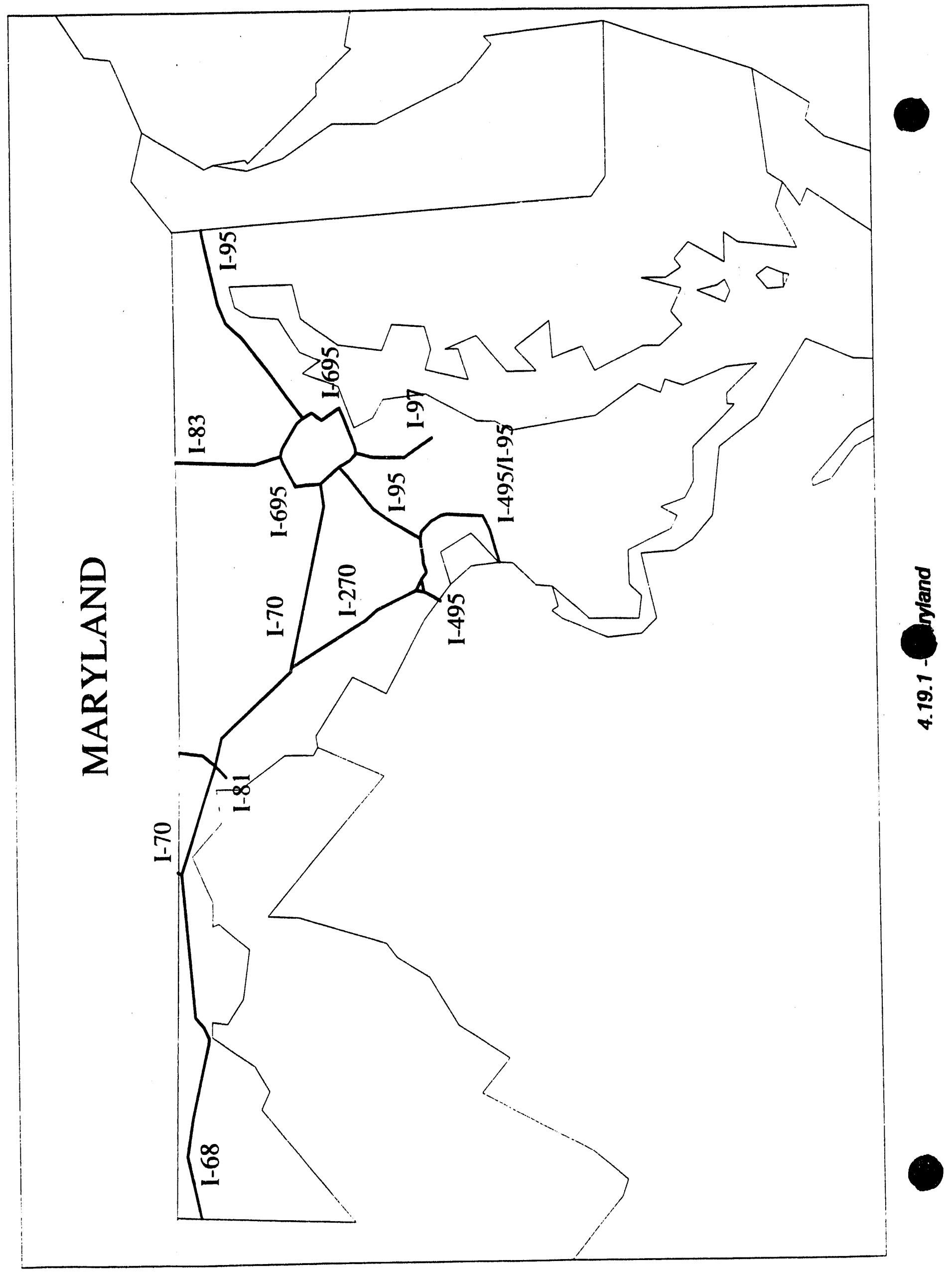




\section{Preferred Routes in Massachusetts}

[-190

1-290

|-291

I-295

1-395

I-495

I-84

1-90)

[-91

I-93

I-95

- From the junction with 1-290 in Worcester to the end of 1-190 near Leominster

- From the junction with 1-395 and I-90 south of Worcester to the junction with 1-495 near Marlborough

- From the junction with I-91 in Springfield to the junction with I-90 northeast of Springfield

- From the Rhode Island state line to the junction with I-95 near Attleboro

- From the Connecticut state line to the junction with I-290 and 1-90 south of Worcester

- From the junction with 1-195 north of Wareham to the junction with I-95 east of Amesbury

- From the Connecticut state line to the junction with 1-90 north of Sturbridge

- From the New York state line to the junction with 1-495 west of Framingham

- From the Connecticut state line to the Vermont state line

- From the junction with I-495 southeast of Lawrence to the New Hampshire state line

- From the junction with 1-295 near Attlesboro to the junction with I-495 south of Foxboro

- From the junction with 1-495 east of Amesbury to the New Hampshire state line

\section{State Designated Preferred Routes}

Massachusetts has not designated any additional roads. 


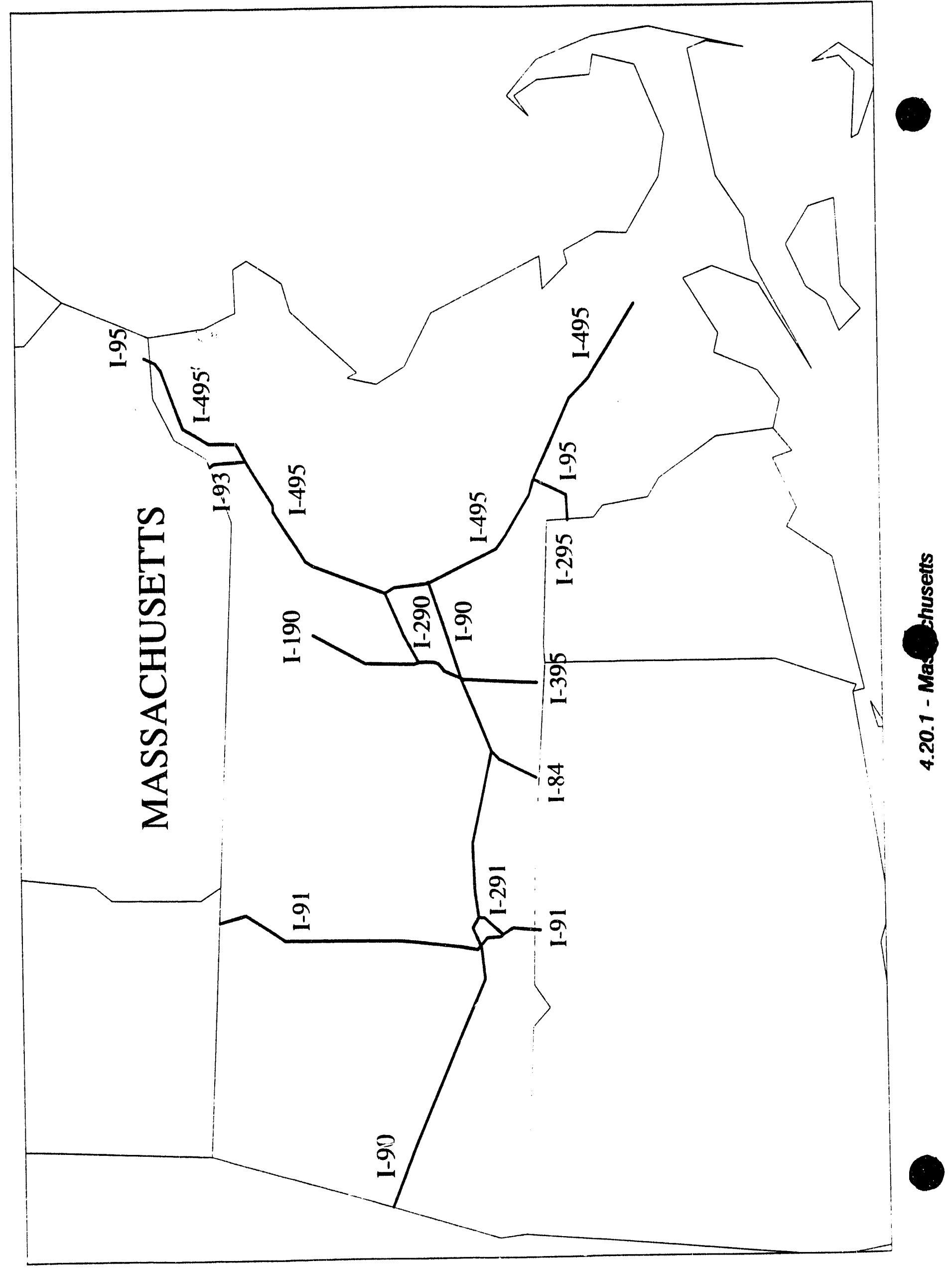




\section{Preferred Routes in Michigan}

- From the junction with I-94 east of Benton Harbor to the junction of 1-96 east of Grand Rapids

- From the junction with 1-75 northeast of Monroe to the junction with I-96 near Plymouth

- From the Indiana state line to the junction with 1-96 southwest of Lansing

- From the junction with 1-96 northeast of Lansing to the junction with 1-94 near Port Huron

From the junction of 1-69 and 1-96 southwest of Lansing to the junction of I-69 and 1-96 northwest of Lansing

- From the Ohio state line to the junction with 1-275 northeast of Monroe

- From the junction with 1-696 north of Detroit to the Canadian border

- From the Indiana state line to the junction with I-275 near Romulus

- From the junction with I-696 in St. Clair Shores to the Canadian horder

- From the Muskegon to the junction with I-69 northwest of Lansing

- From the junction with 1-69 southwest of Lansing to the junction with 1-275 and 1-696 in Farmington Hills

\section{State Designated Preferred Routes}

Michigan has not designated any additional roads. 


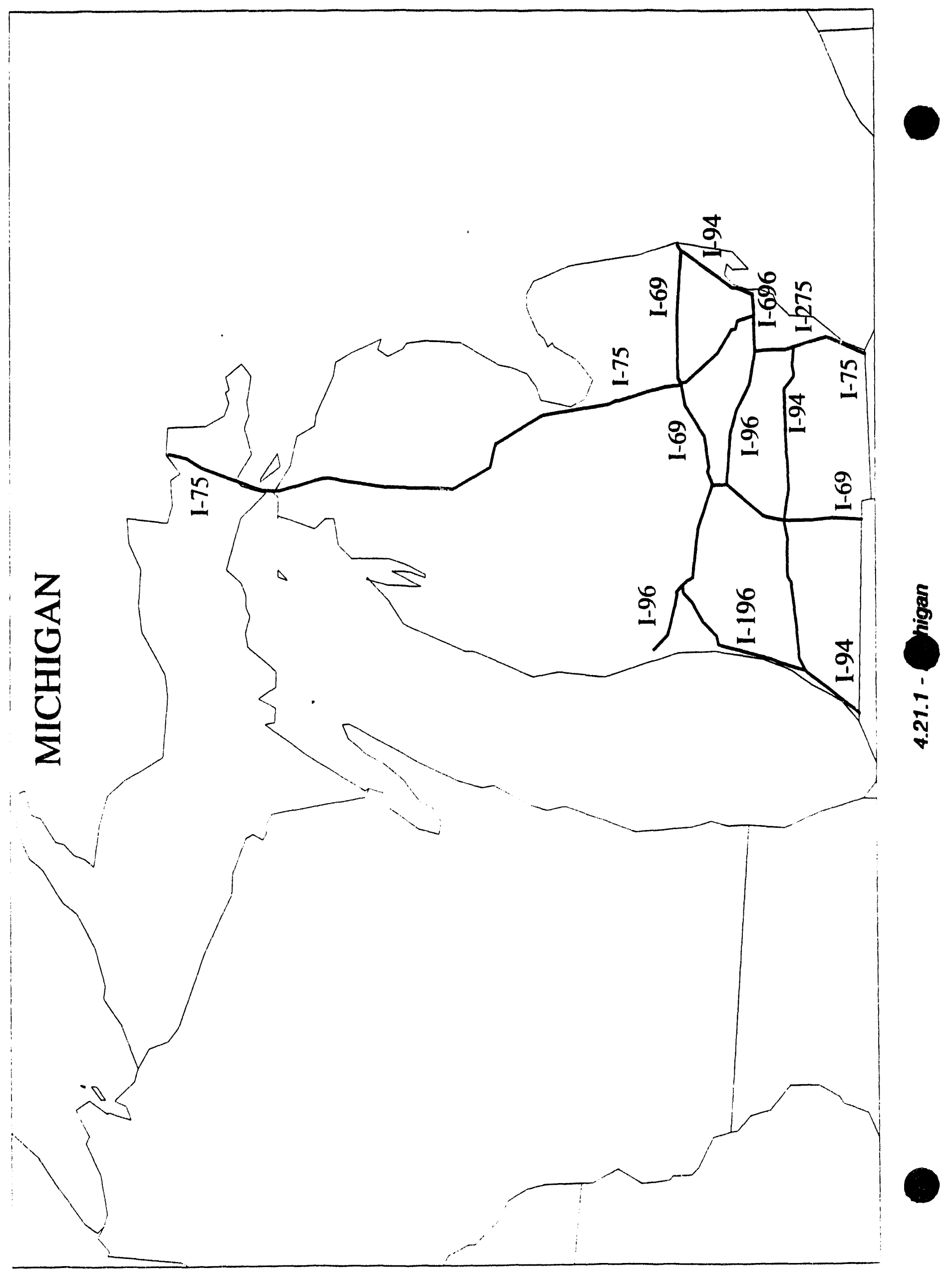




\section{Preferred Routes in Minnesota}

I-35

- From the lowa state line to the junction of 1-35E/l-35W in Apple Valley

- $\mathrm{nm}$ the junction of I-35E/l-35W northeast of Lino Lakes to the end of $\mathrm{I}-35$ in Duluth

I-35E

- From the junction with I-35/I-35W in Apple Valley to the junction with I-494 in Mendota Heights

- From the junction with I-694 southwest of White Bear Lake to the junction of I-35/l-35W northeast of Lino Lakes

I-35W

- From the junction with 1-35/1-35E in Apple Valley to the junction with 1-494 in Bloomington

- From the junction with I-694 in New Brighton to the junction of I-35/I-35E northeast of Lino Lakes

- From the junction of 1-94 in Maple Grove to the junction of I-694 and I-94 east of St. Paul

- From the junction of I-35 in Duluth to the Wisconsin state line

- From the junction with I-494 and I-94 east of St. Paul to the junction with 1-94 in Brooklyn Center

- From the South Dakota state line to the Wisconsin state line

- From the North Dakota state line to the junction with I-694 in Brooklyn Center

- From the junction with I-494 and 1-694 east of St. Paul to the Wisconsin state line

\section{State Designated Preferred Routes}

Minnesota has not designated any additional roads. 


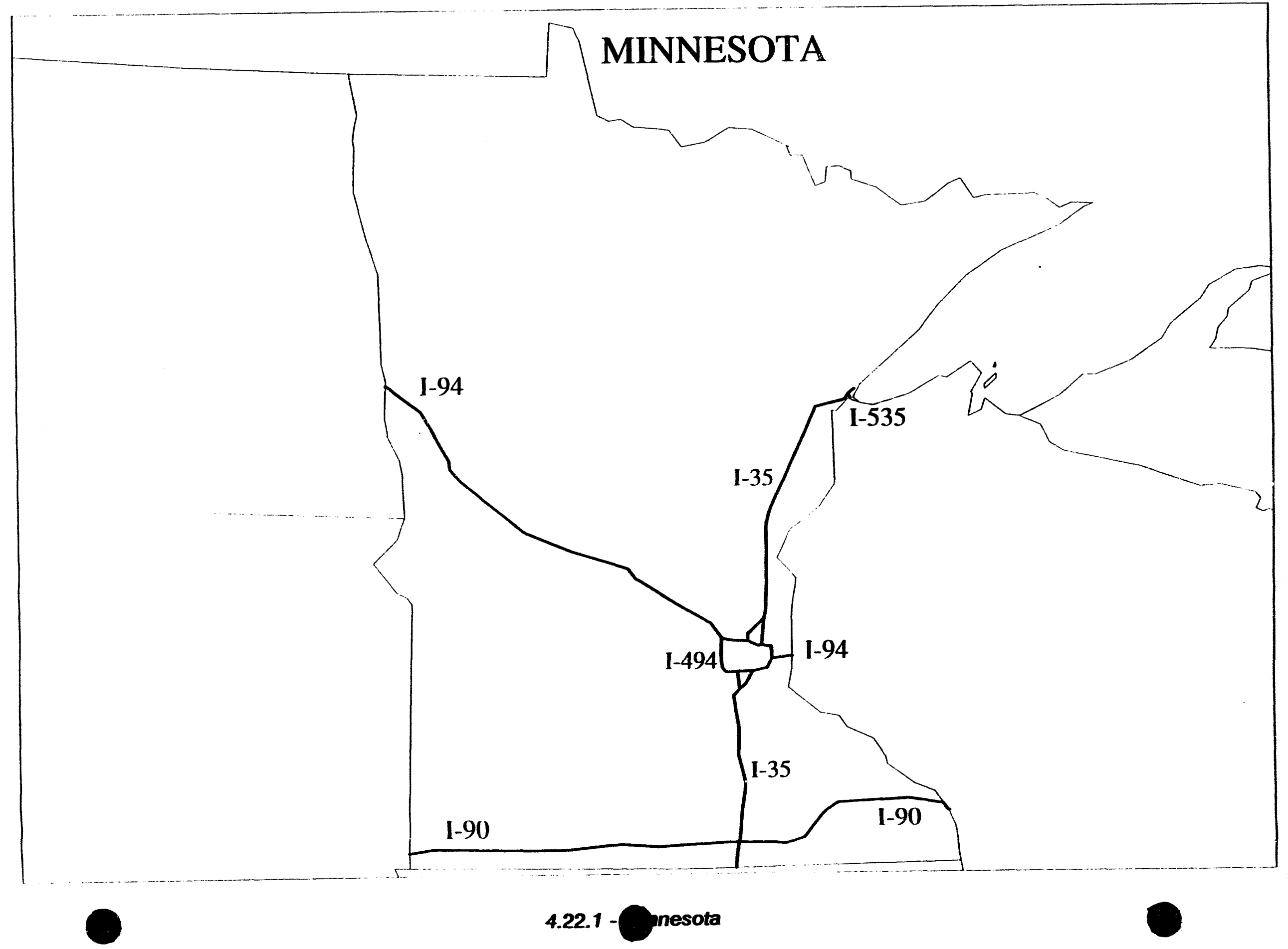




\section{Preferred Routes in Mississippi}

1-20 From the Louisiana state line to the junction of I-55 southwest of Jackson

- From the junction with I-55 south of Jackson to the junction with I-59 west of Meridian

I-2(0/I-55 - From the junction with I-20 and I-55 southwest of Jackson to the junction with I-20 and I-55 south of Jackson

1-20/1-59 - From the junction with I-20 and I-59 west of Meridian to the Alabama state line

- From the junction with 1-55 north of Jackson to the junction with I-20 west of Jackson

I-55 - From the Louisiana state line to the junction with I-20 southwest of Jackson

- From the junction with I-20 south of Jackson to the Louisiana state line

$1-59$

- From the Louisiana state line to the junction with 1-20 west of Meridian

I-10 From the Louisiana state line to the Alabama state line

\section{State Designated Preferred Routes}

Mississippi has not designated any additional roads. 


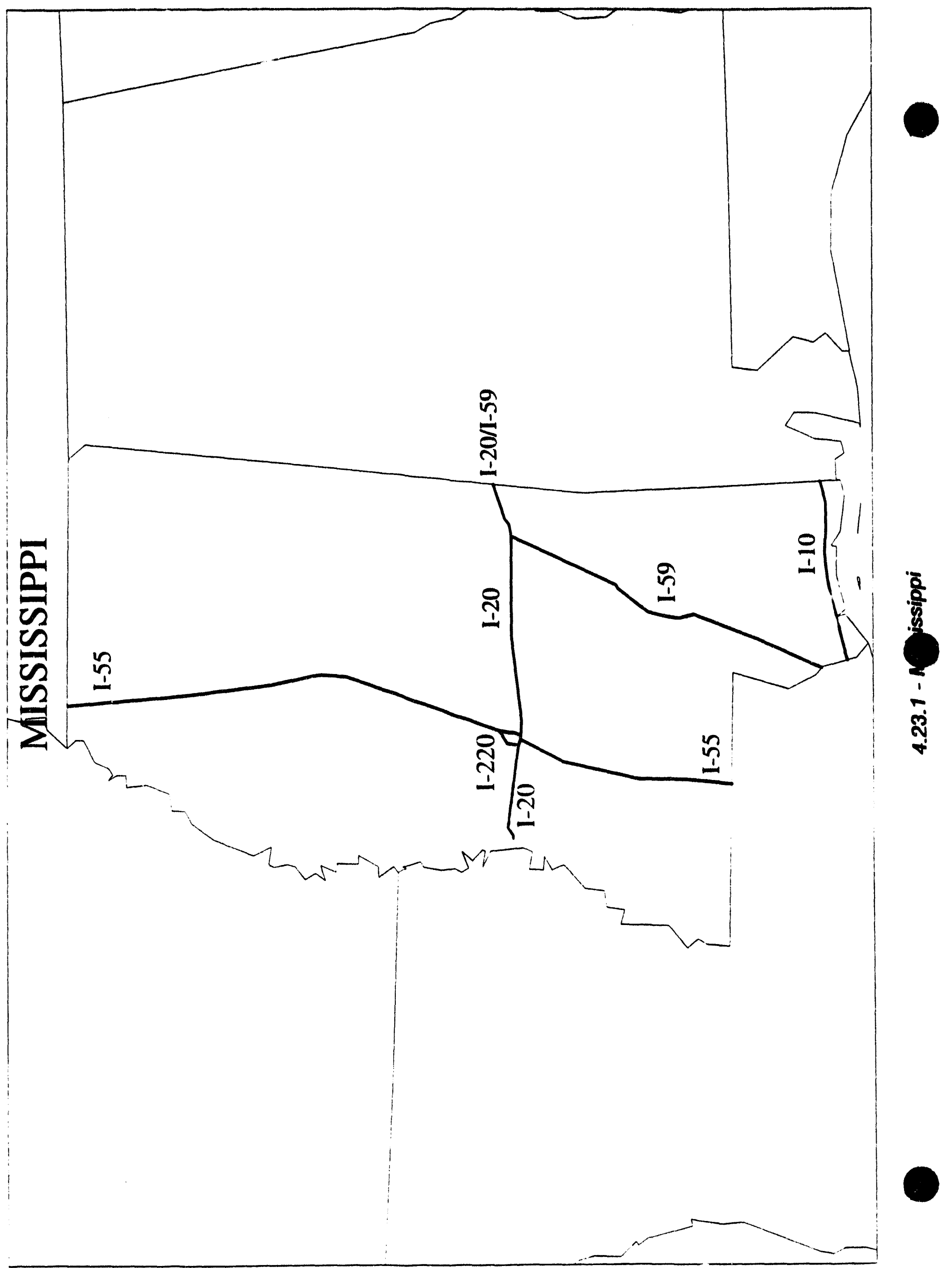




\section{○}

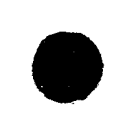

\section{Preferred Routes in Missouri}

I-155 - From the junction with 1-55 near Caruthersville to the Tennessee state line

I-255 - From the junction with I-55 and I-270 south of St. Louis to the Illinois state line

I-270 - From the junction with 1-55 and 1-255 south of St. Louis to the Illinois state line

1-29 - From the junction with 1-435 near the Kansas City International Airport to the Iowa state line

1-35 From the junction with 1-435 northeast of Kansas City to the lowa state line

I-435 - From the junction with I-29 northwest of Kansas City to the Kansas state line

- From the junction with 1-29 northwest of Kansas City to the Kansas state line east, and southern portions of the metropolitan area)

I-44 From the Oklahoma state line to the junction of I-270 southwest of St. Louis

1-470 - From the junction with 1-435 south of Kansas City to the junction with 1-70 near Independence

1-55 - From the Arkansas state line to the junction with 1-270 and 1-255 south of St. Louis

I-57 - From the Illinois state line to the junction with 1-55 near Sikeston

1-70 - From the junction with 1-470 near Independence to the junction with 1-270 northwest of St. Louis

\section{State Designated Preferred Routes}

Missouri has not designated any additional roads. 

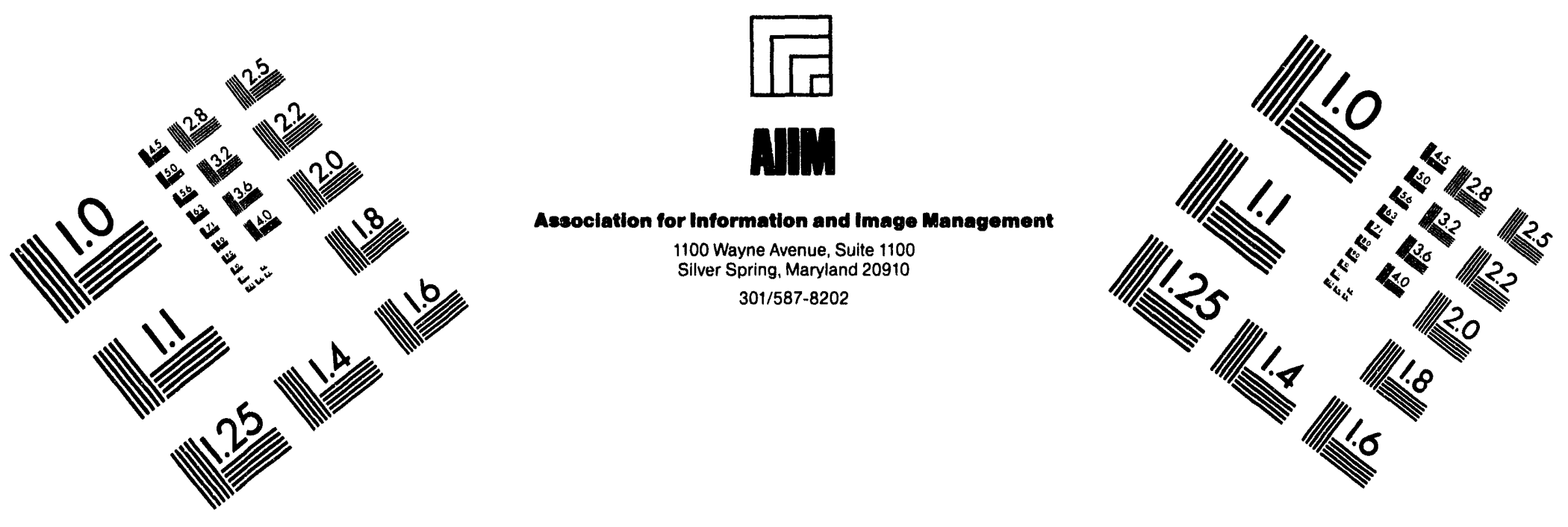

\section{Centimeter}

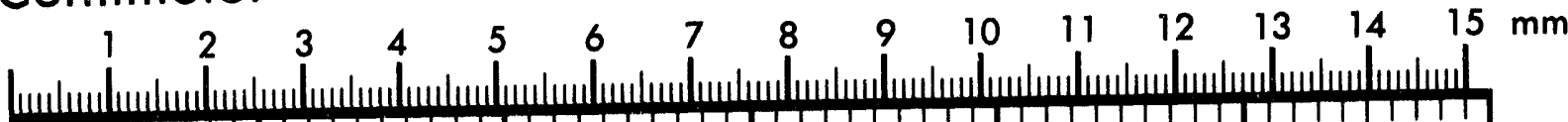

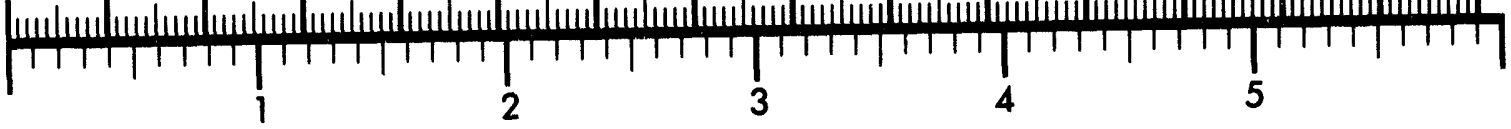
Inches
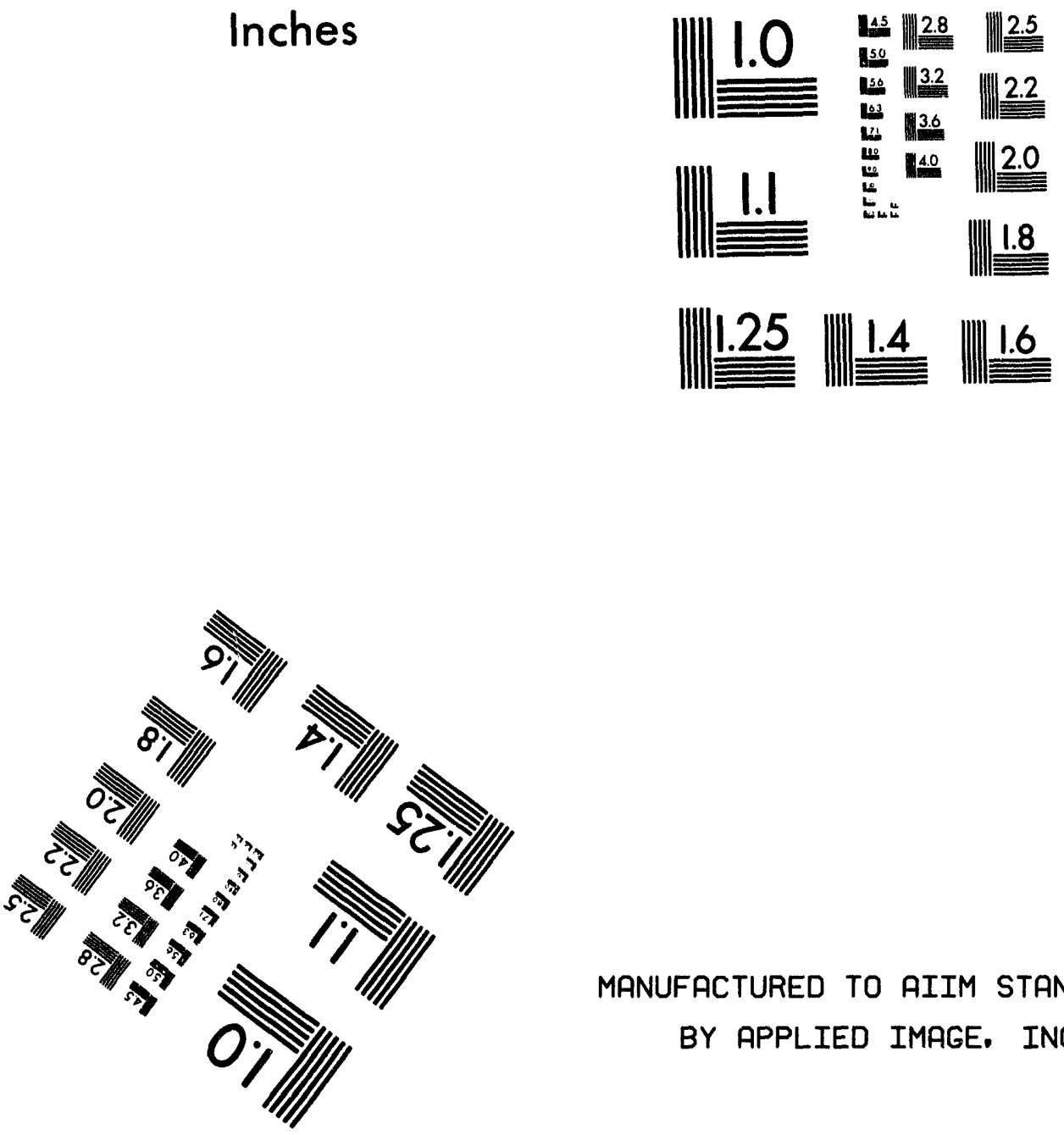

MANUFACTURED TO AIIM STANDARDS

BY APPLIED IMAGE, INC.

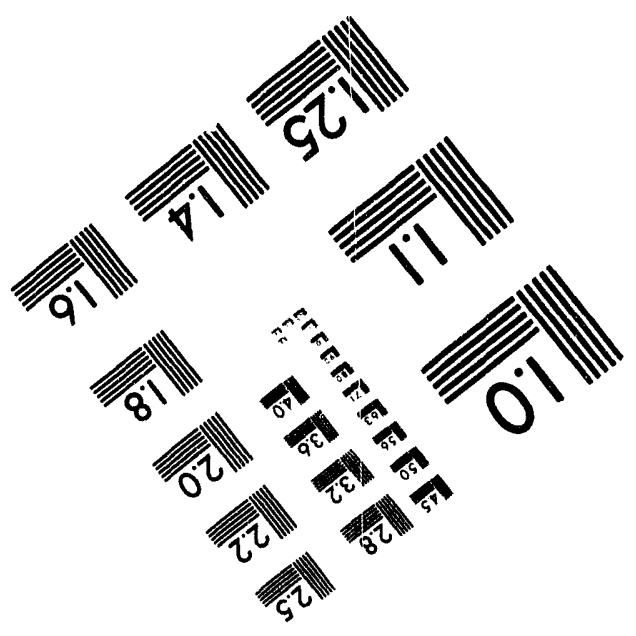



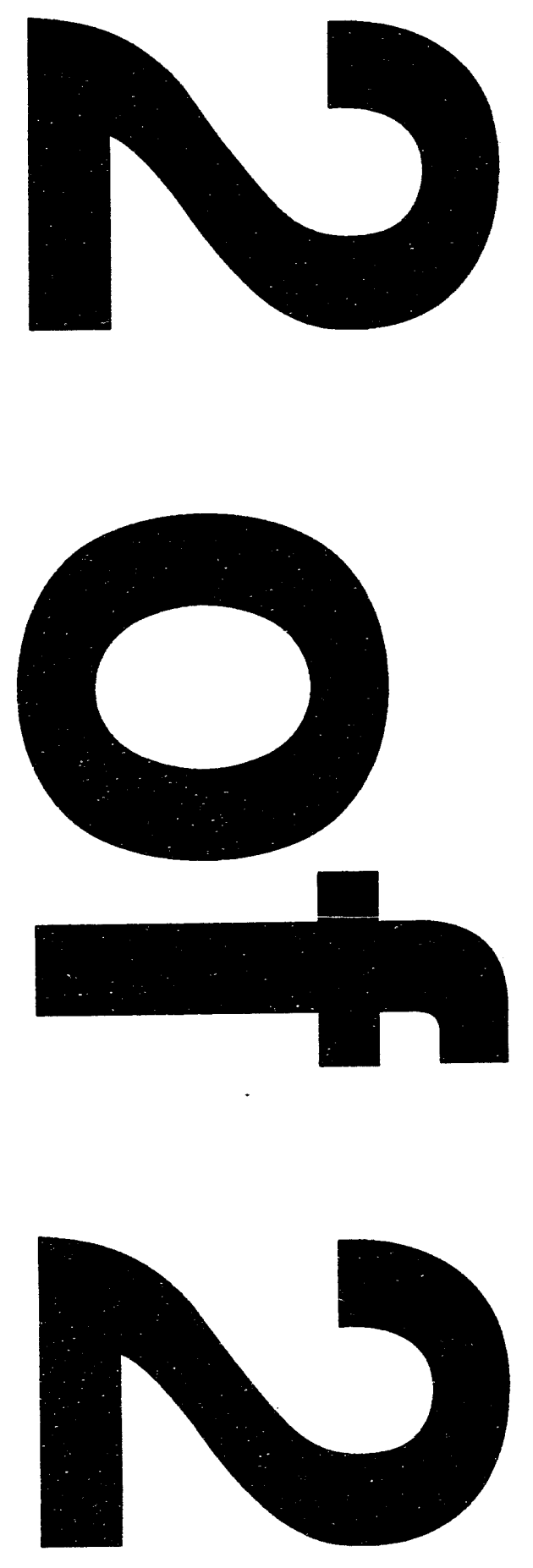


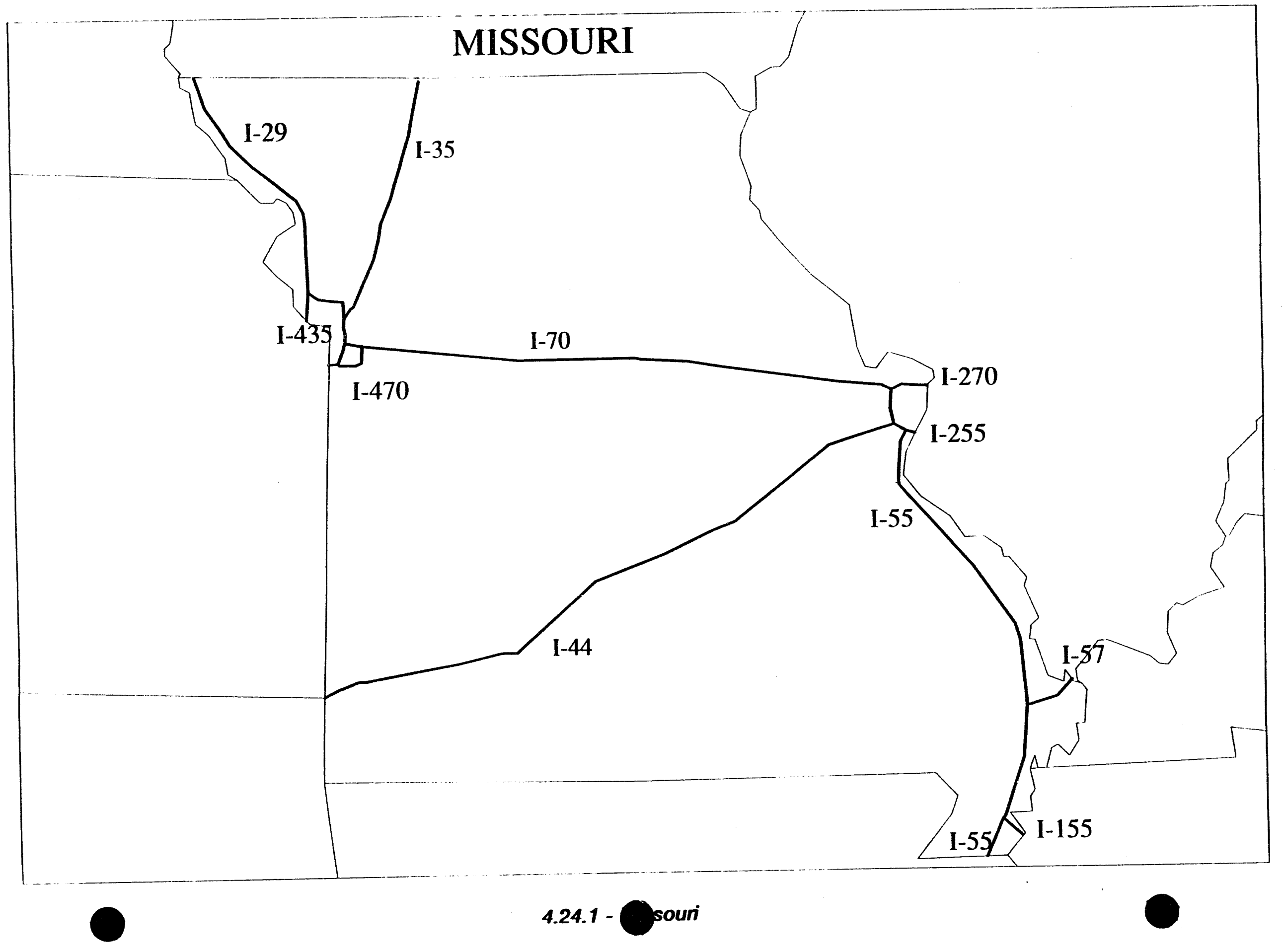



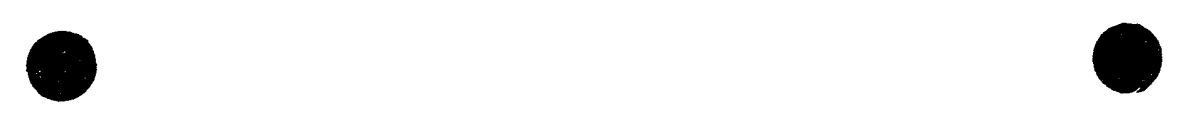

\section{Preferred Routes in Montana}

I-15

- From the Idaho state line to junction with I-90 west of Butte

- From the junction with I-90 east of Butte to the Canadian border

I-15/I-90 - From the I-15/I-90 junction west of Butte to the I-15/I-90 junction east of Butte

- From the Idaho state line to the junction with I-15 west of Butte

- From the junction with I-15 east of Butte to the Wyoming state line

I-94 From the North Dakota state line to I-90 near Billings

\section{State Designated Preferred Routes}

Montana has not designated any additional roads. 


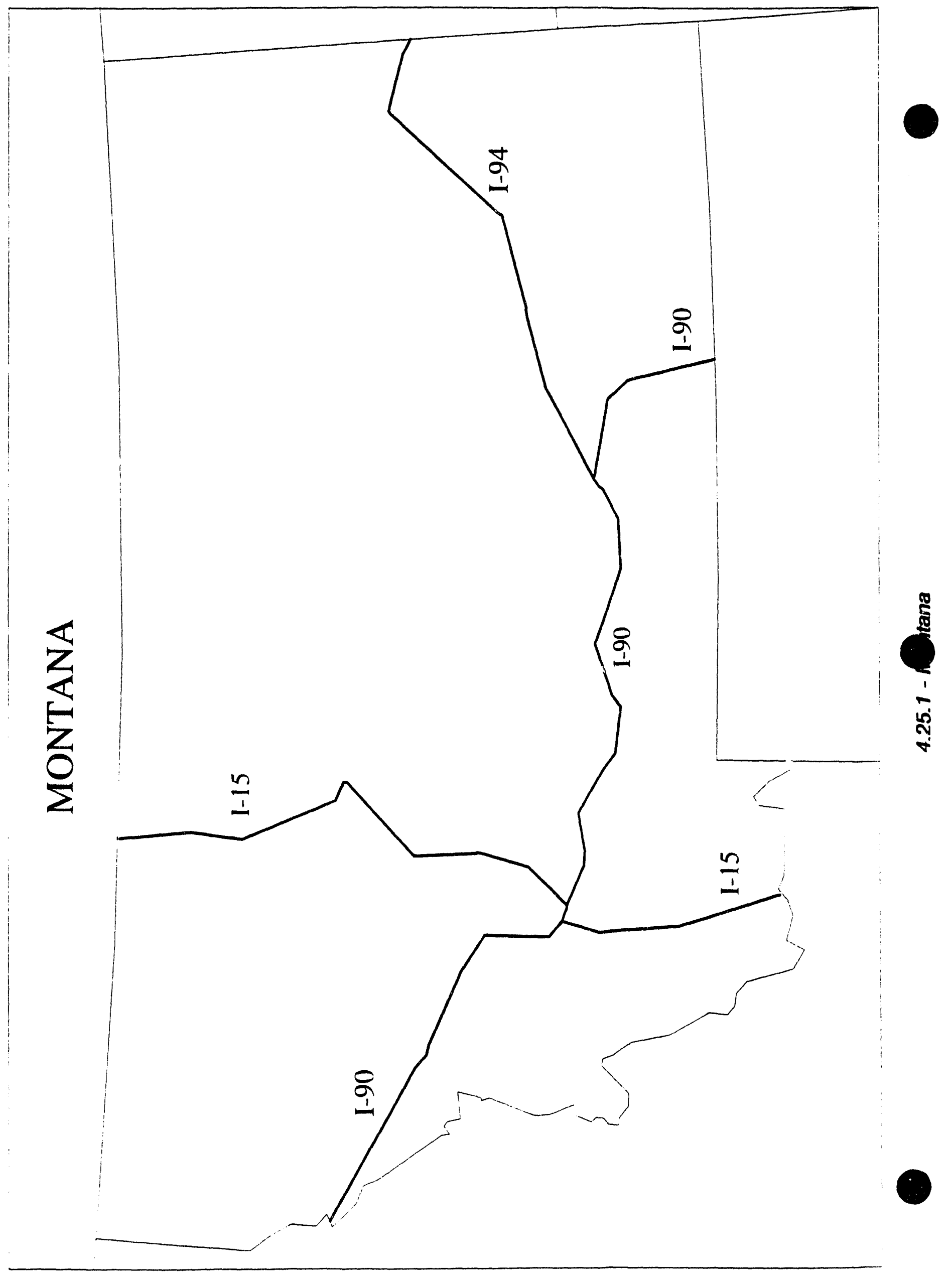




\section{Preferred Routes in Nebraska}

1-129 - From the lowa state line to the end of 1-129 near Dakota City

1-680 - From the Iowa state line to the junction with I-80 west of Omaha

I-76 - From the Colorado state line to the junction with I-80

I-80 - From the Wyoming state line to the junction with I-680 west of Omaha

\section{State Designated Preferred Routes}

Nebraska designated, on August 3, 1988, that I-680 be used in lieu of $I 80$ in the Omaha area. 


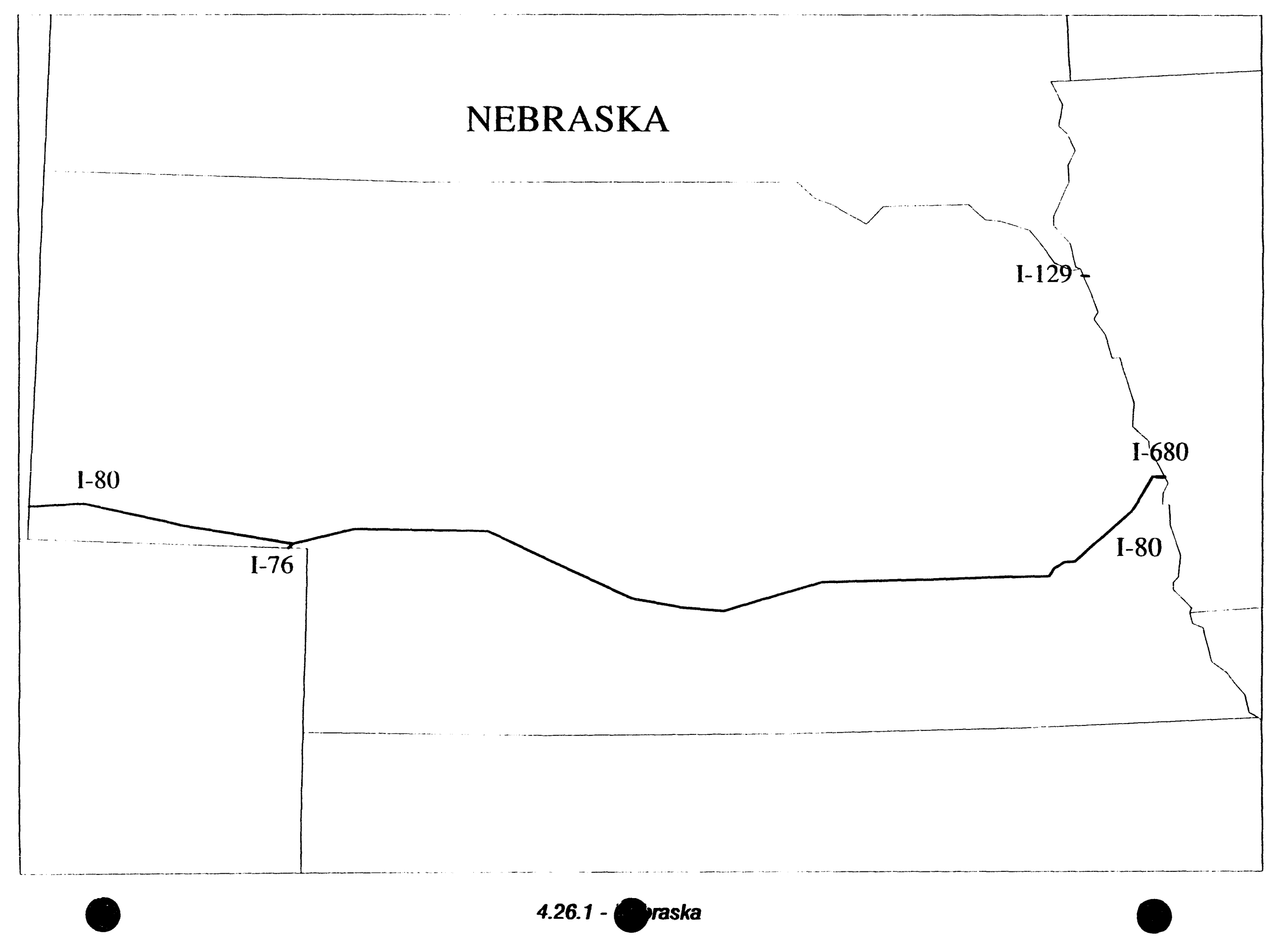




\section{Preferred Routes in Nevada}

I-15 - From the Arizona state line to the California state line

I-80 From the California state line to the Utah state line

\section{State Designated Preferred Routes}

Nevada has not designated any additional roads. 


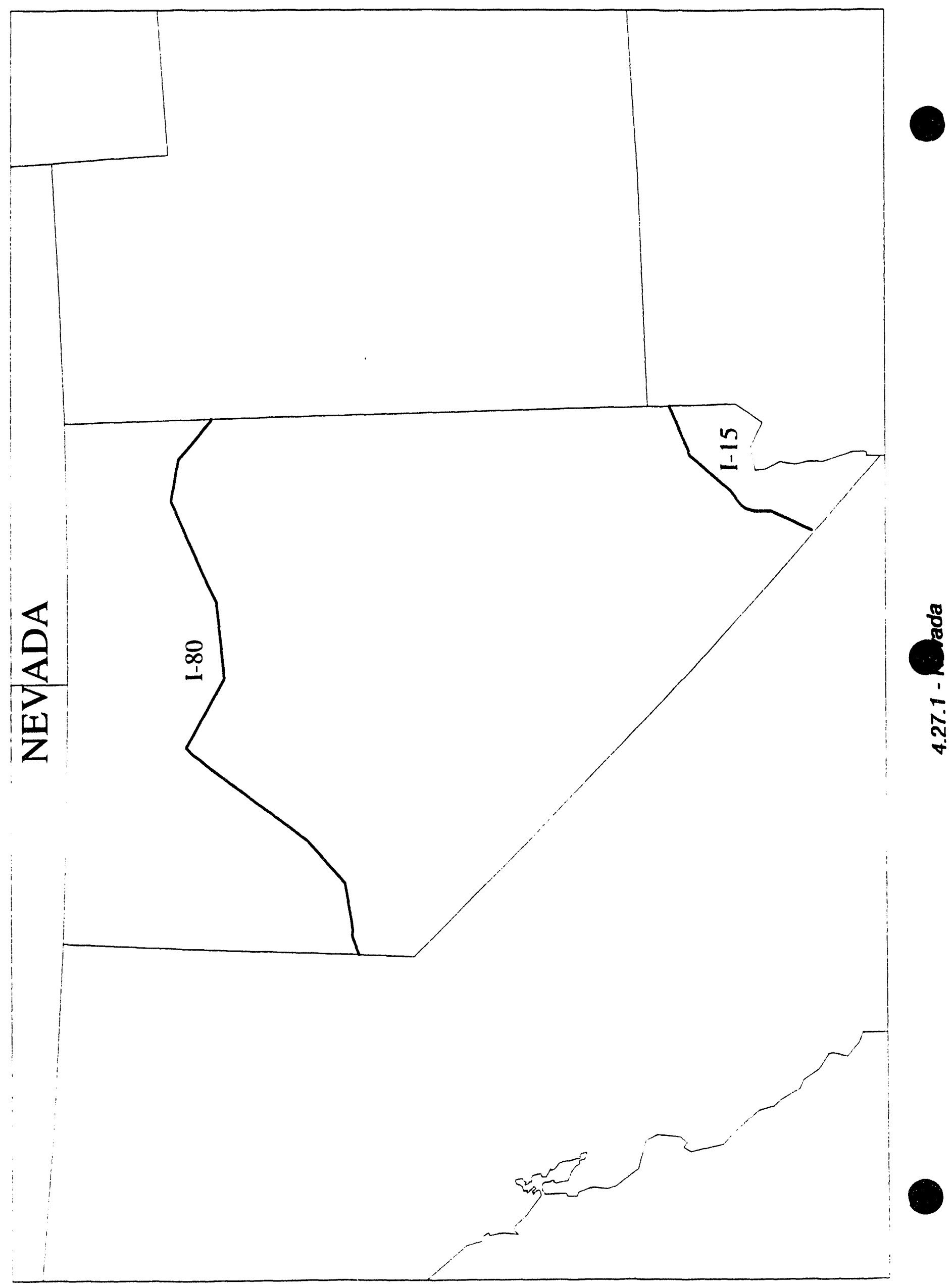



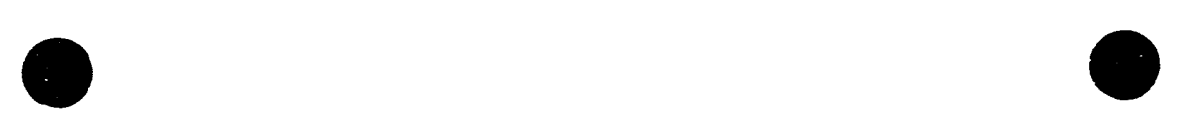

\section{Preferred Routes in New Hampshire}

1-89

- From the junction with 1-93 south of Concord to the Vermont state line

$1-93$

- From Massachusetts state line to the Vermont state line

I-95

- From the Massachusetts state line to the Maine state line

\section{State Designated Preferred Routes}

New Hampshire has not designated any additional roads. 


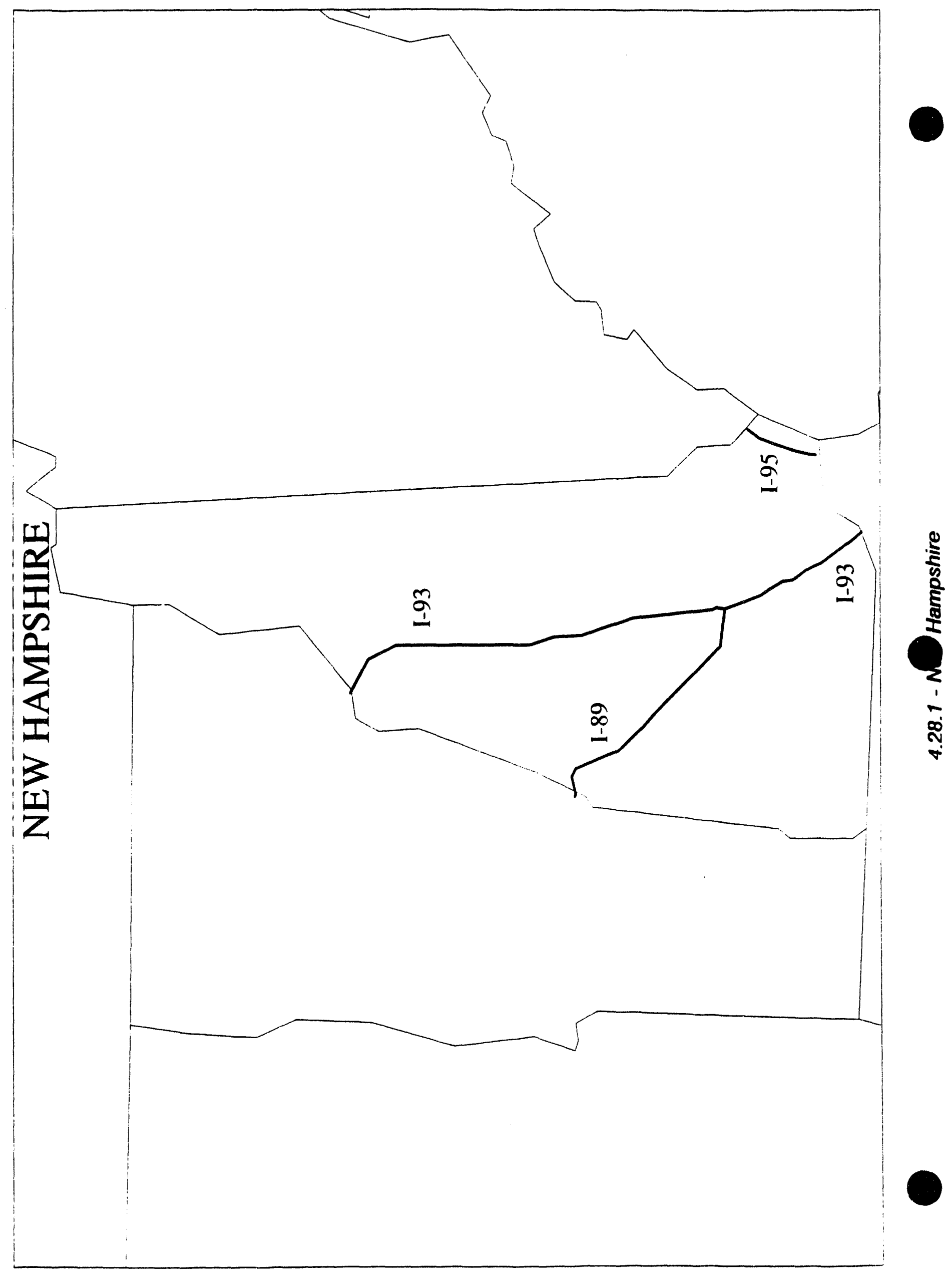




\section{Preferred Routes in New Jersey}

- From the junction with I-295 south of Trenton to the end of I-195 southwest of Neptune

- From the Pennsylvania state line to the junction with the New Jersey Turnpike

- From the Delaware state line to the junction with U.S. 130 south of Trenton

- From the junction with I-195 south of Trenton to the junction with I-95 north of Trenton

- U.S. 130 and U.S. 206 provide temporary connection for the portion of 1-295 which is under construction south of Trenton

- From the junction with the New Jersey Turnpike west of Perth Amboy to the junction with I-80 near Parsippany

- From the Pennsylvania state line to the junction with I-287

I-80)

- From the Pennsylvania state line to the New York state line The New Jersey Turnpike between I-287 and I-276 serves as a temporary connection for the uncompleted portion of I-95
between Trenton and New Brunswick.

\section{State Designated Preferred Routes}

New Jersey has not designated any additional roads. 


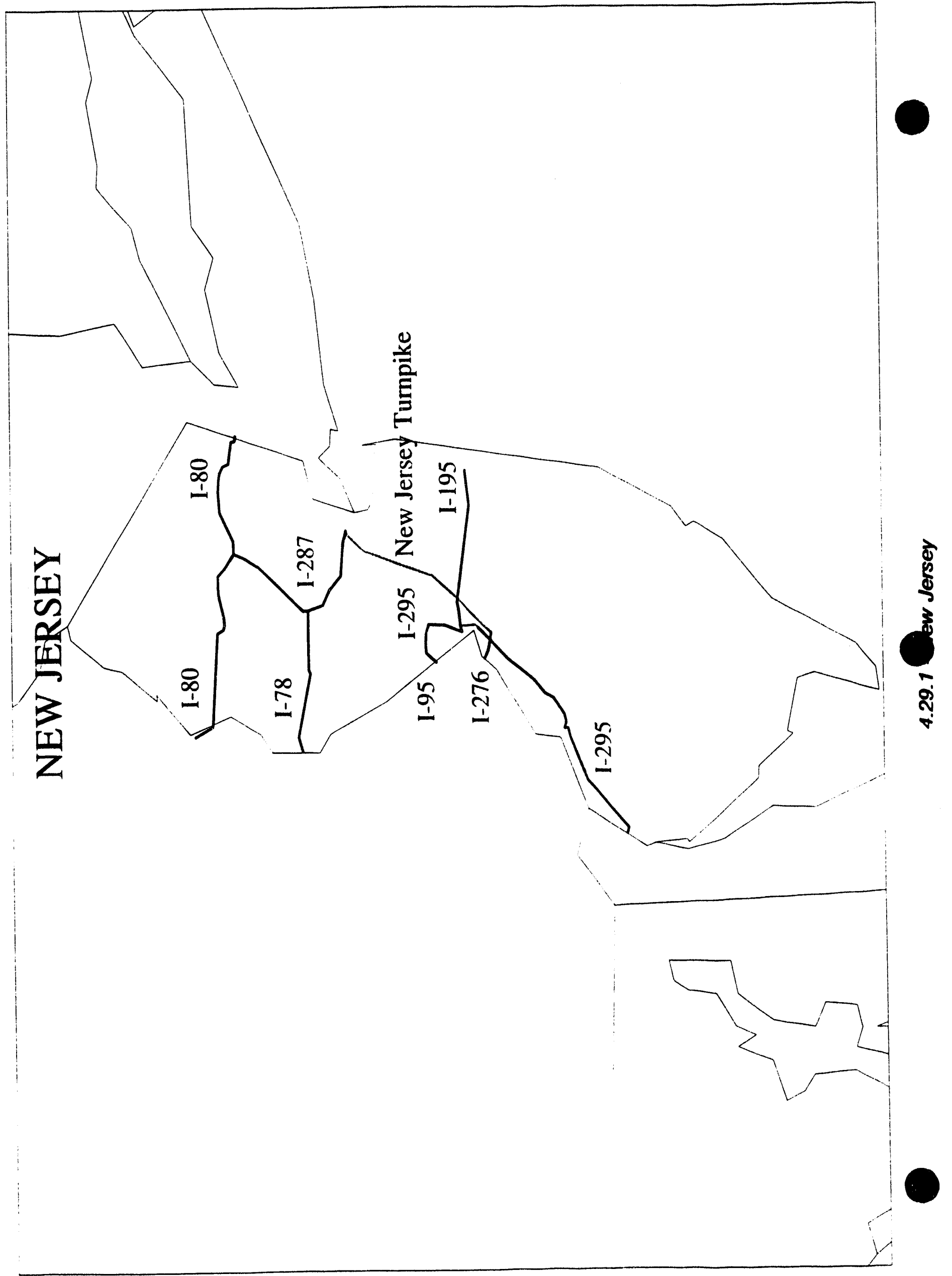




\section{Preferred Routes in New Mexico}

1-10

- From the Arizona state line to the Texas state line

I-25

- From the junction with 1-10 in Las Cruces to the Colorado state line

I-40

- From the Arizona state line to the Texas state line

\section{State Designated Preferred Routes}

New Mexico has designated the following roads for shipment to the Waste Isolation Pilot Plant (WIPP). These roads can not he used by other HRCQ shipments.

U.S. 180/62 - From the junction with U.S. 285 to the WIPP access road

U.S. 285 - From the junction with $1-25$ southeast of Santa Fe to the Texas state line

U.S. 285/84 - From the junction with S.R. 502 to the junction with I-25 south of Santa Fe

U.S. 54

- From the junction with I-41) near Santa Rosa to U.S. 285 near Vaughn

S.R. 4

- From S.R. 502 to Los Alamos Truck Route

S.R. 502

- From S.R. 4 to U.S. 285/84

Los Alamos Truck Route - From Los Alamos to S.K. 4 


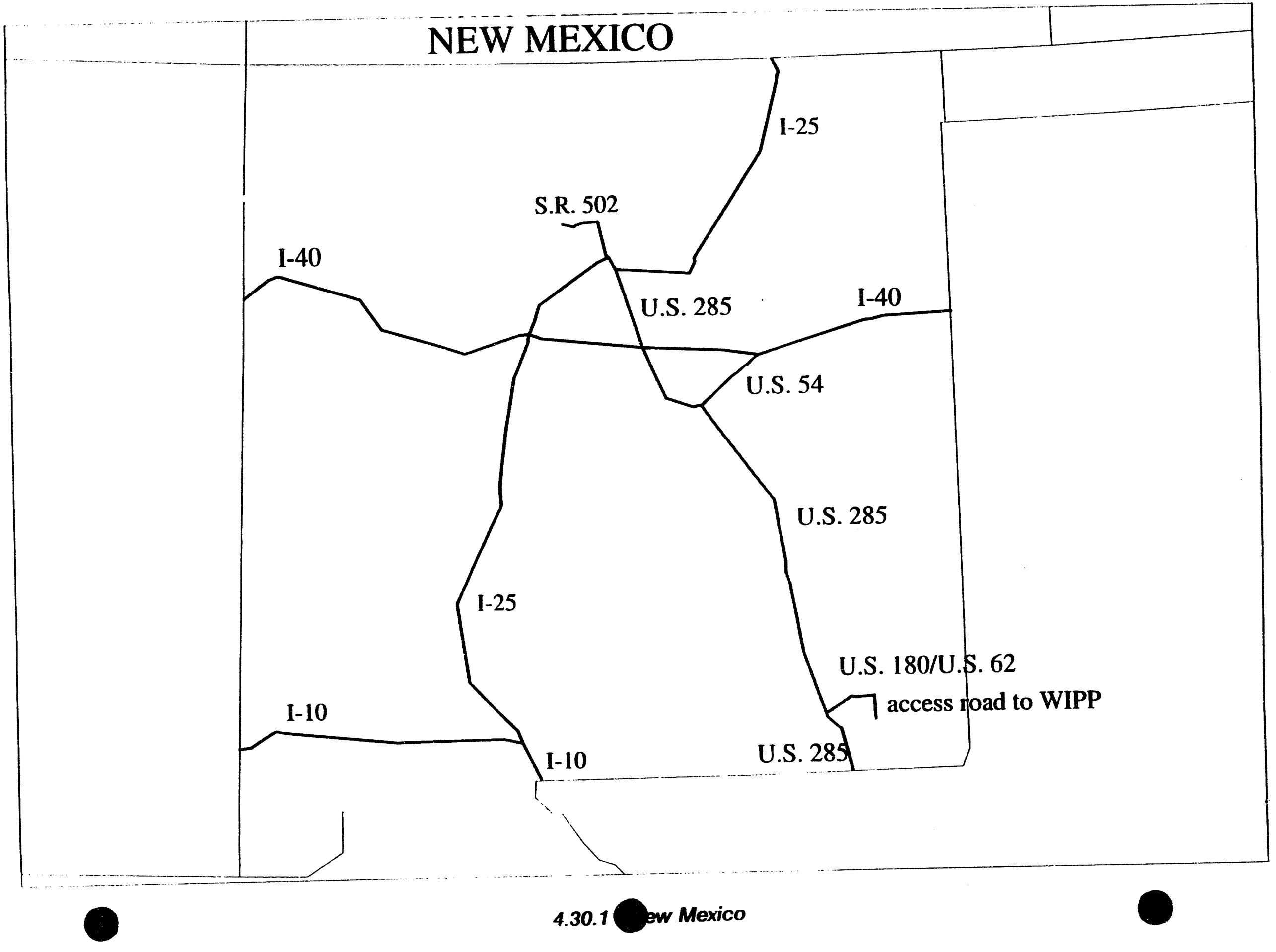




\section{Preferred Routes in New York}

I-190

1-287

I-287/I-87

$1-290$

1-295

1-390

$1-481$

1-490)

I-495

$1-590$

1-684

$1-81$

I-84

$1-87$

I-88

$1-90$

$1-95$

- From the junction with 1-290 northwest of Buffalo to the end of 1-190 north of Niagara Falls

- From the junction with I-87 west of White Plains to the junction with I-95 near Port Chester

- From the junction with I-87 near Suffern to the junction with 1-87 west of White Plains

- From the junction with I-90 northeast of Buffalo to the junction with I-190 north of Buffalo

- From the junction with I-95 in Bronx to the junction with I-495 in Queens

- From the junction with $[-490$ west of Rochester to the end of $1-390$ northwest of Bath

- From the junction with 1-81 south of Syracuse to the junction with 1-81 north of Syracuse

- From the junction with 1-90 southwest of Rochester to the junction with 1-390 west of Rochester

- From the junction with 1-590 east of Rochester to the junction with I-90 southeast of Rochester

- From the junction with I-295 in Queens to the end of I-495 near Calverton

- From the junction with 1-390 south of Rochester to the junction with 1-490 east of Rochester

- From the junction with 1-287 in White Plains to the Connecticut state line

- From the Connecticut state line to the junction with I-684 near Brewster

- From the Pennsylvania state line to the junction with 1-481 south of Syracuse

- From the junction with 1-90 north of Syracuse to the Canadian border

- From the Pennsylvania state line to the Connecticut state line

- From the junction with 1-95 in Bronx to the junction with 1-287 west of White Plains

- From the junction with 1-287 near Suffern to the Canadian border

- From the junction with 1-81 north of Binghamton to the junction with 1-90 west of Schenectady

- From the Pennsylvania state line to the Massachusetts state line

- From the New Jersey state line to the Connecticut state line

\section{State Designated Preferred Routes}

New York has not designated any additional roads. 


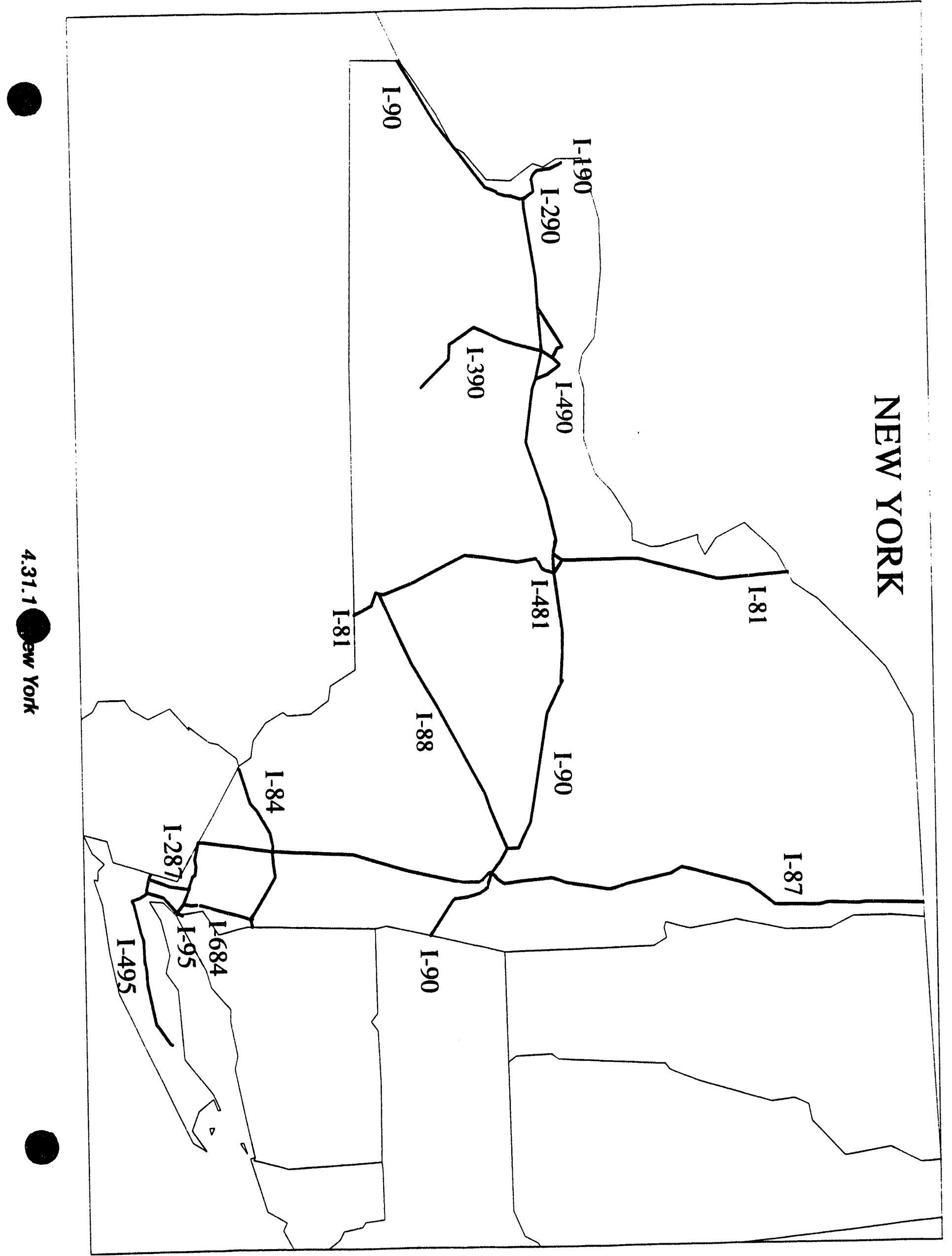




\section{Preferred Routes in North Carolina}

I-26

- From the South Carolina state line to the junction with I-40 southwest of Asheville

1-40 - From the Tennessee state line to the junction with 1-85 west of Winston-Salem

- From the junction with 1-85 west of Durham to the end of 1-40 northeast of Wilmington

1-40/1-85 - From the junction with I-40 and I-85 west of Winston-Salem to the junction with I-40 and I-85 west of Durham

- From the South Carolina state line to the Virginia state line

I-85 - From the South Carolina state line to the junction with I-40 west of Winston-Salem

- From the junction with I-85 west of Durham to the Virginia state line

$1-95$

- From the South Carolina state line to the Virginia state line

\section{State Designated Preferred Routes}

North Carolina has not designated any additional roads. 


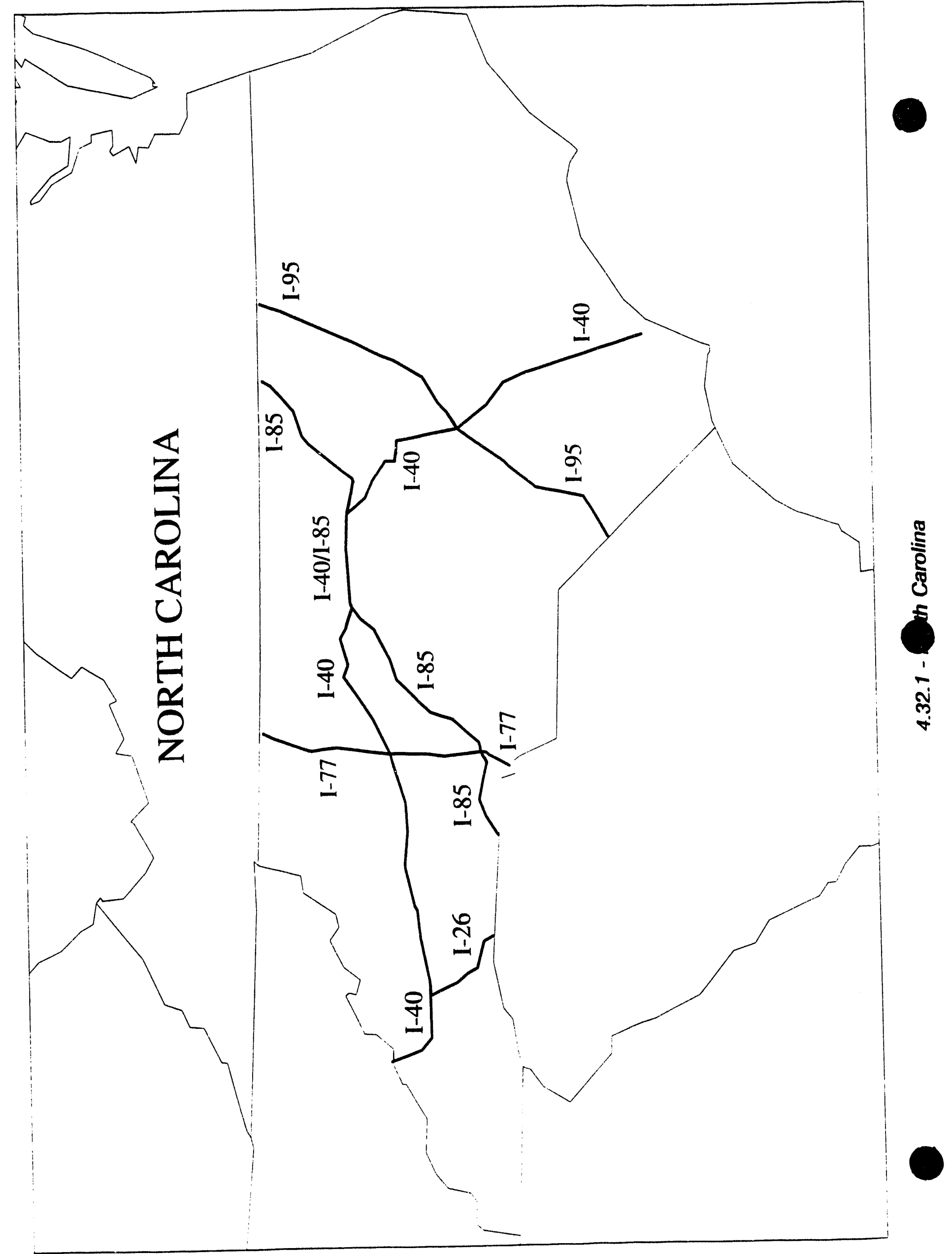




\section{Preferred Routes in North Dakota}

1-29 From the South Dakota state line to the Canadian border

1-94 From the Minnesota state line to the Montana state line

\section{State Designated Preferred Routes}

North Dakota has not designated any additional roads. 
NORTH DAKOTA

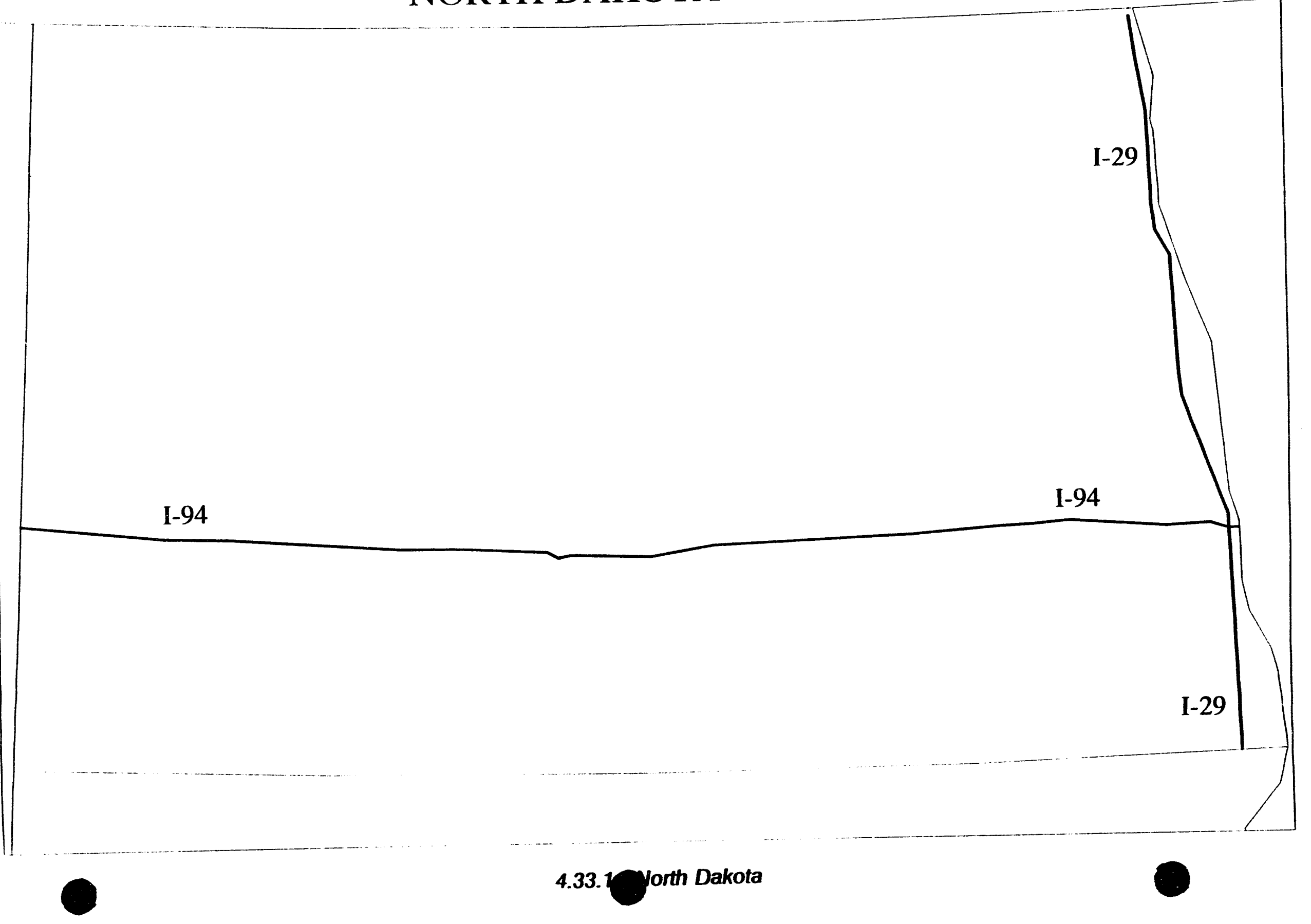




\section{Preferred Routes in Ohio}

1-270 Entire route around Columbus

$[-271$

$1-275$

1-277

1-280

$[-470$

I-475

$1-480$

1-675

$1-70$

$[-7 !$

I-74

$1-75$

$1-76$

$1-77$

1-80

- From junction with I-90 west of Cleveland to the Pennsylvania state line

$\begin{array}{lll}1-80 & \text { - From junction with I-90 west of Cleveland to the Pennsylvania state line } \\ 1-8(0 / 1-90 & \text { - From the Indiana state line to junction of } I-80 \text { and } 1-90 \text { west of Cleveland }\end{array}$

1-90 - From 1-271 northeast of Cleveland to the Pennsylvania state line

- From 1-71 south of Cleveland metro area to I-90 northeast of Cleveland

- From the Indiana state line to the Kentucky state line around the Cincinnati area

- From I-76 to I-77 in Akron

- From 1-80/1-90 to 1-75 east of Tuledo

- From I-70 to the West Virginia state line

- From I-75 near Perrysburg to I-75 in Toledo

- From 1-80 southwest of the Cleveland metro area to I-80 southeast of Cleveland

- From I-75 south of Dayton to I-70 near Fairborn

- From the Indiana state line to I-270 west of Columbus

- From 1-270 east of Columbus to I-470 west of Bellaire

- From I-275 northeast of Cincinnati to I-270 south of Columbus

- From I-270 north of Columbus to 1-480 southwest of Cleveland

- From the Indiana state line to I-275 northwest of Cincinnati

- From 1-270 north of Cincinnati to 1-80/1-90 near Toledo

- From 1-475 north of Toledo to the Michigan state line

- From I-71 west of Akron to the Pennsylvania state line

- From I-71 the West Virginia state line to I-480 south of Cleveland

\section{State Designated Preferred Routes}

Ohio has not designated any additional roads. 


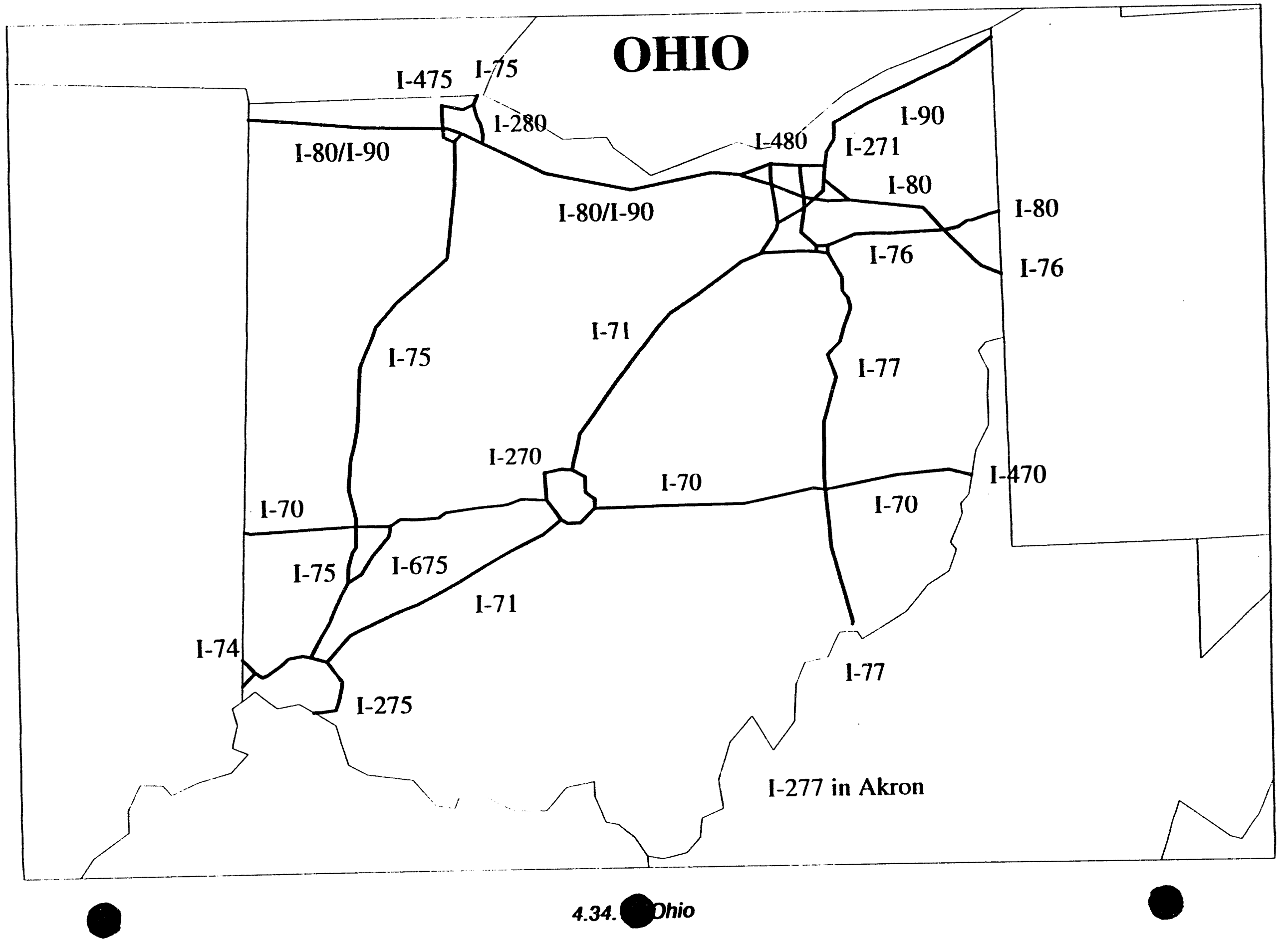




\section{Preferred Routes in Oklahoma}

1-240 - From I-40 southeast of the Oklahoma City metro area to I-44 southwest of Oklahoma City

1-35 - From the Texas state line to the junction with 1-240 south of Oklahoma City

- From the junction with I-40 east of Oklahoma City to the junction with I-44 northeast of Oklahoma City

- From the junction with I-44 southeast of Edmond to the Kansas state line

1-35/1-44 - From northeast of Oklahoma City to southeast of Edmond

1-40 - From the Texas state line to I-44 west of Oklahoma City

- From the junction of 1-35 east of Oklahoma City to the Arkansas state line

I-44 From the Texas state line to the junction with I-35 northeast of Oklahoma City

- From the junction with 1-35 southeast of Edmond to the Missouri state line

\section{State Designated Preferred Routes}

Oklahoma has not designated any additional roads. 


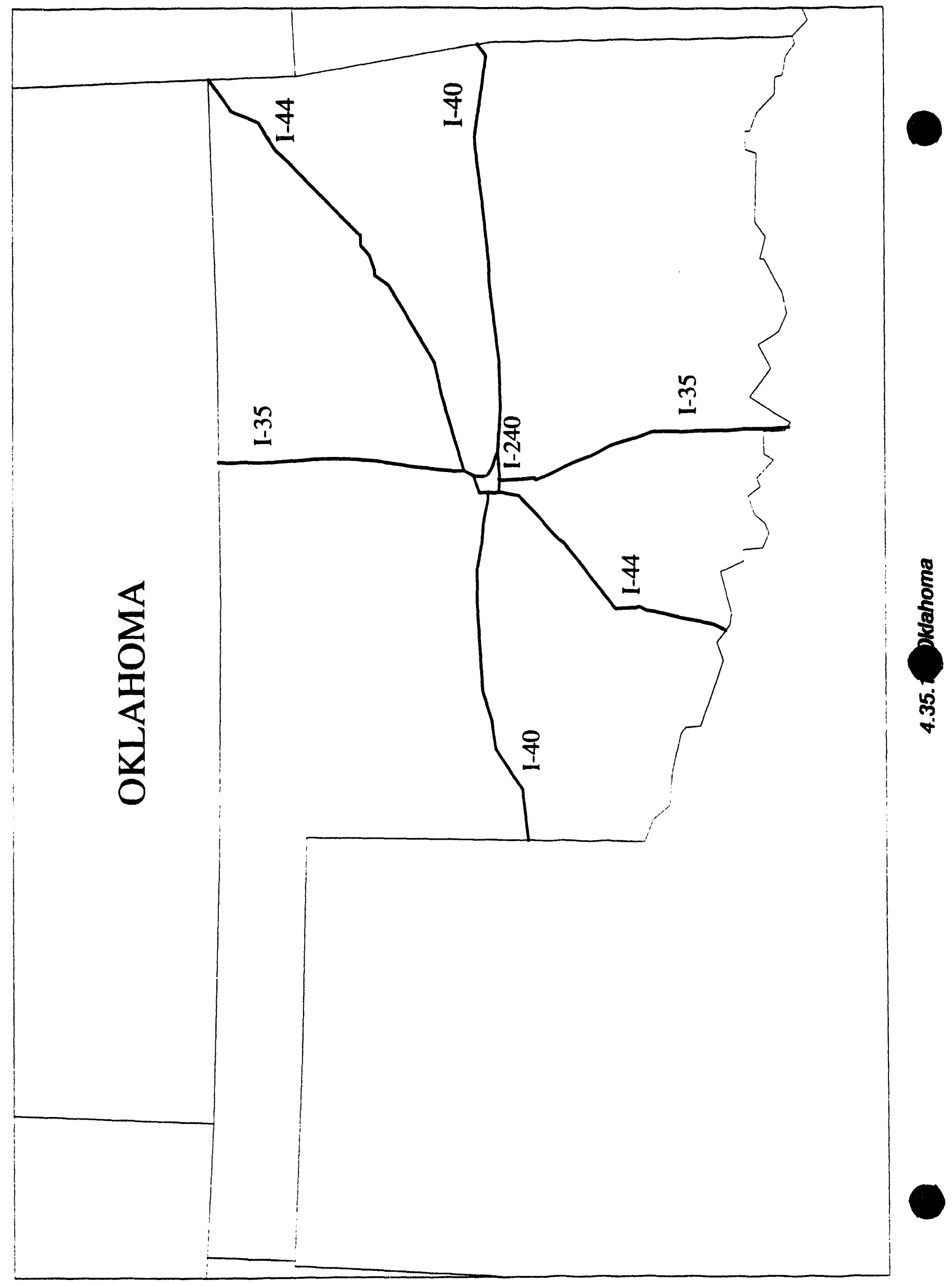



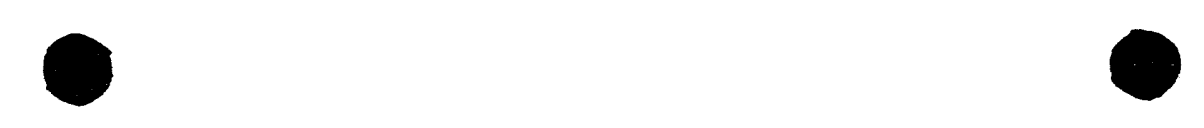

\title{
Preferred Routes in Oregon
}

\author{
I-105 - From I-5 to Eugene \\ 1-205 - From the Washington state line to I-5 south of Portland \\ I-5 From the junction with I-205 south of Portland to the California state line \\ 1-82 - From Washington state line to 1-84 \\ I-84 From I-205 to Idaho state line
}

\section{State Designated Preferred Routes}

Oregon has not designated any additional roads. 


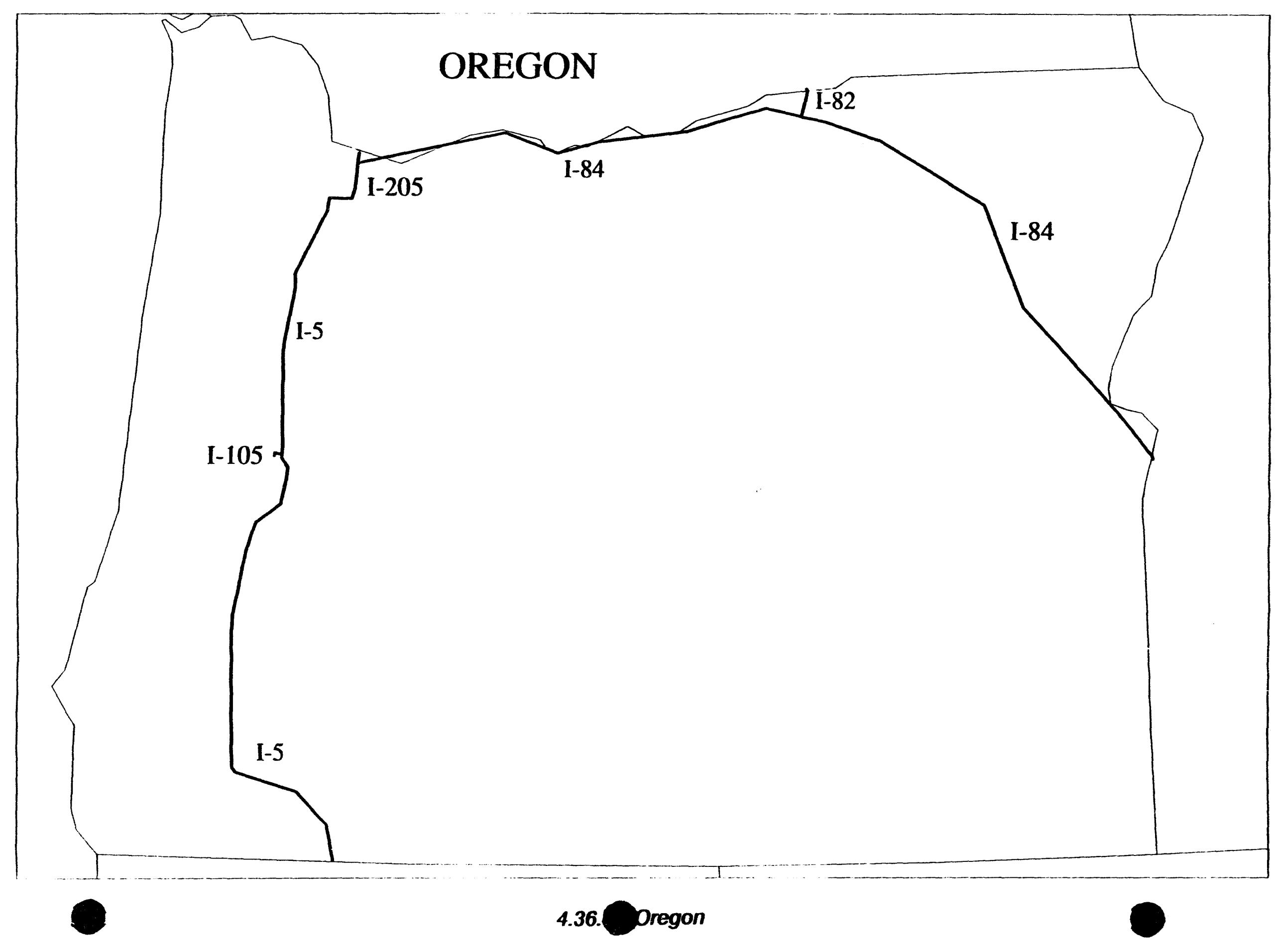




\section{Preferred Routes in Pennsylvania}

I-176 - From I-76 to the end if I-176 southeast of Reading

1-180 - From 1-80 north of Milton to Williamsport

1-276 - From the junction of 1-76 near King of Prussia to the New Jersey state line

$1-283$

$1-380$

- From the junction of 1-76 southeast of Harrisburg to the junction of 1-83 east of Harrisburg

- From the junction with 1-84 southeast of Scranton to the junction with 1-80

$\mathrm{I}-380 / 1-84$ From the junction with 1-81 near Dunmore to the split of these roads southeast of Scranton

1-476 - From the junction of 1-95 near Chester to the junction of 1-276 east of Norristown

1-70 - From the West Virginia state line to the junction with 1-79 north of Washington

- From the junction with 1-79 east of Washington to the junction with 1-76 southwest of Greenburg

- From the junction with 1-76 at Breezewood to the Maryland state line

1-70/1-76 - From the junction of these roads southwest of Greenburg to Breezewood

$1-70 / 1-79$ - From the junction of these roads north of Washington to east of Washington

I-76 - From the Ohio state line to the junction of I-70 southwest of Greenburg

- From the junction of 1-70 at Breezewood to the junction of 1-476 southwest of Conshohocken

1-78 - From the junction of 1-81 northwest of Lebanon to New Jersey state line

1-79 From the West Virginia state line to the junction with $1-70$ east of Washington

- From the junction with 1-70 north of Washington to Erie

1-80 - From the Ohio state line to New Jersey state line

I-81 - From the Maryland state line to New York state line

I-83 - From the Maryland state line to the junction with I-81 northeast of Harrishurg

I-84 From the junction with 1-380 southeast of Scranton to New York state line

$1-90$ - From the Ohio state line to New York state line

1-95 From the Delaware state line to the junction of 1-476 near Chester

- From the Delaware state line to the junction of $1-476$ near Chester $1-276$ and $1-95$ and U.S. 1 has not heen designated as a preferred route)

\section{State Designated Preferred Routes}

Pennsylvania has not designated any additional roads. 


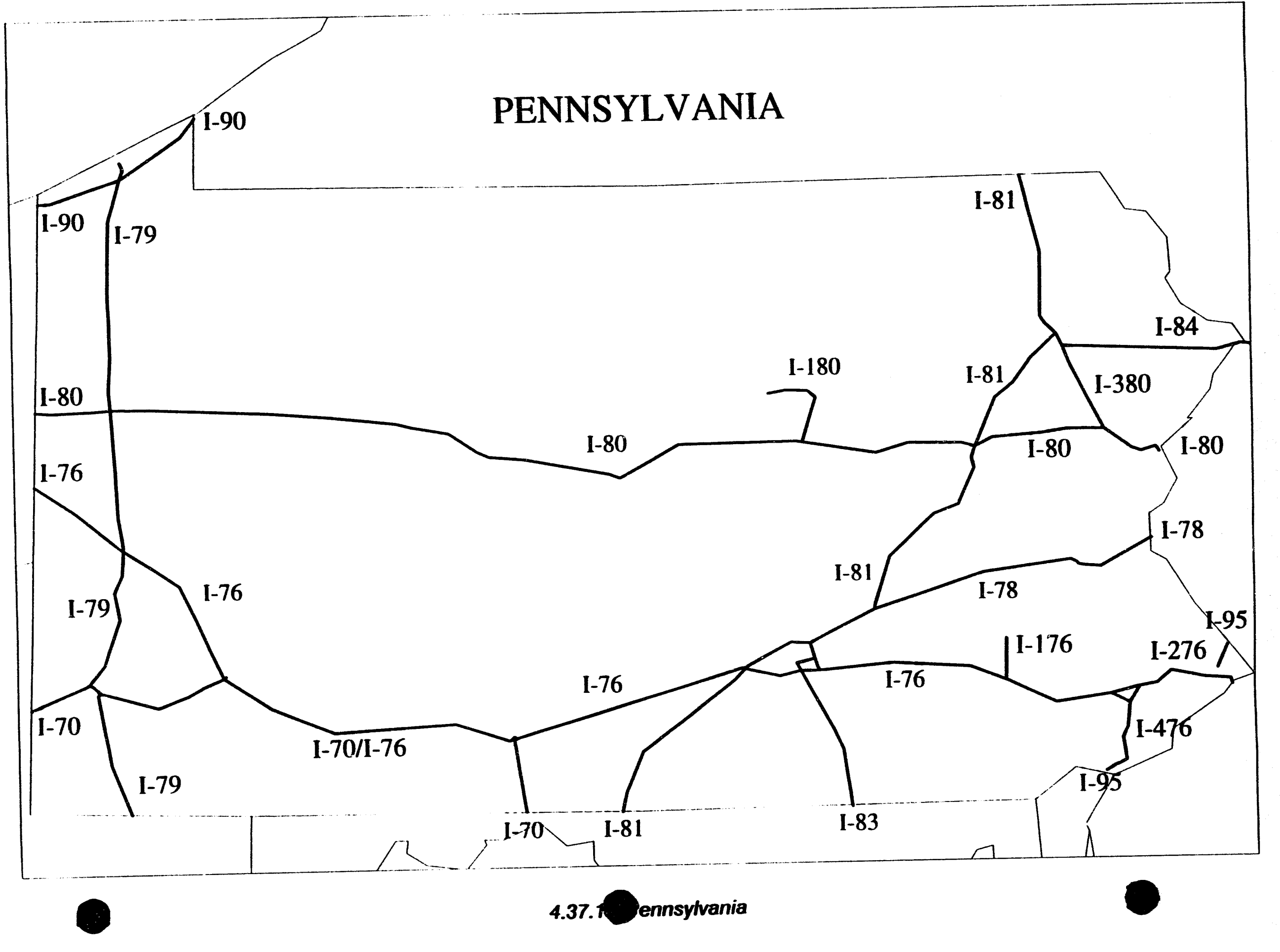




\section{-}

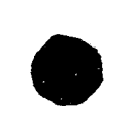

\section{Preferred Routes in Rhode Island}

1-295 From the junction with I-95 southwest of Providence to the Massachusetts state line

I-95 - From the Connecticut state line to the junction with 1-295 southwest of Providence

\section{State Designated Preferred Routes}

Rhode Island has not designated any additional roads. 


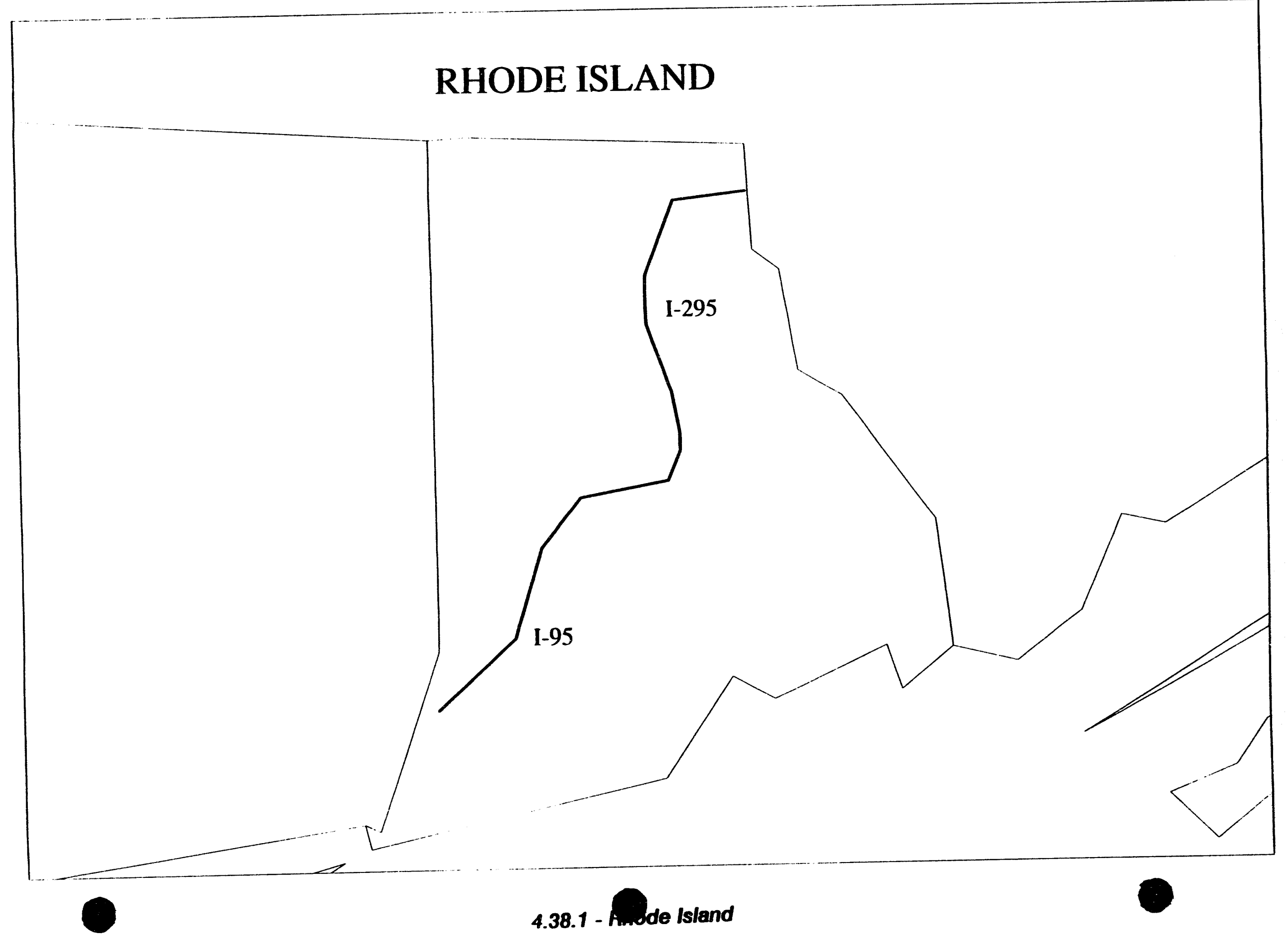




\section{Preferred Routes in South Carolina}

I-20 - From the Georgia state line to the junction with I-95 west of Florence

I-26 From the junction with I-526 north of Charleston to the North Carolina state line

I-385 From the junction with I-26 near Clinton to the junction with I-85 east of Greenville

1-77 - From the junction with 1-26 south of Columbia to the junction with State Route 12 east of Columbia - From Exit 15 northeast of Columbia to the North Carolina state line

I-85 From the Georgia state line to the North Carolina state line

$1-95$

- From the Georgia state line to the North Carolina state line

\section{State Designated Preferred Routes}

South Carolina has not designated any additional roads. 


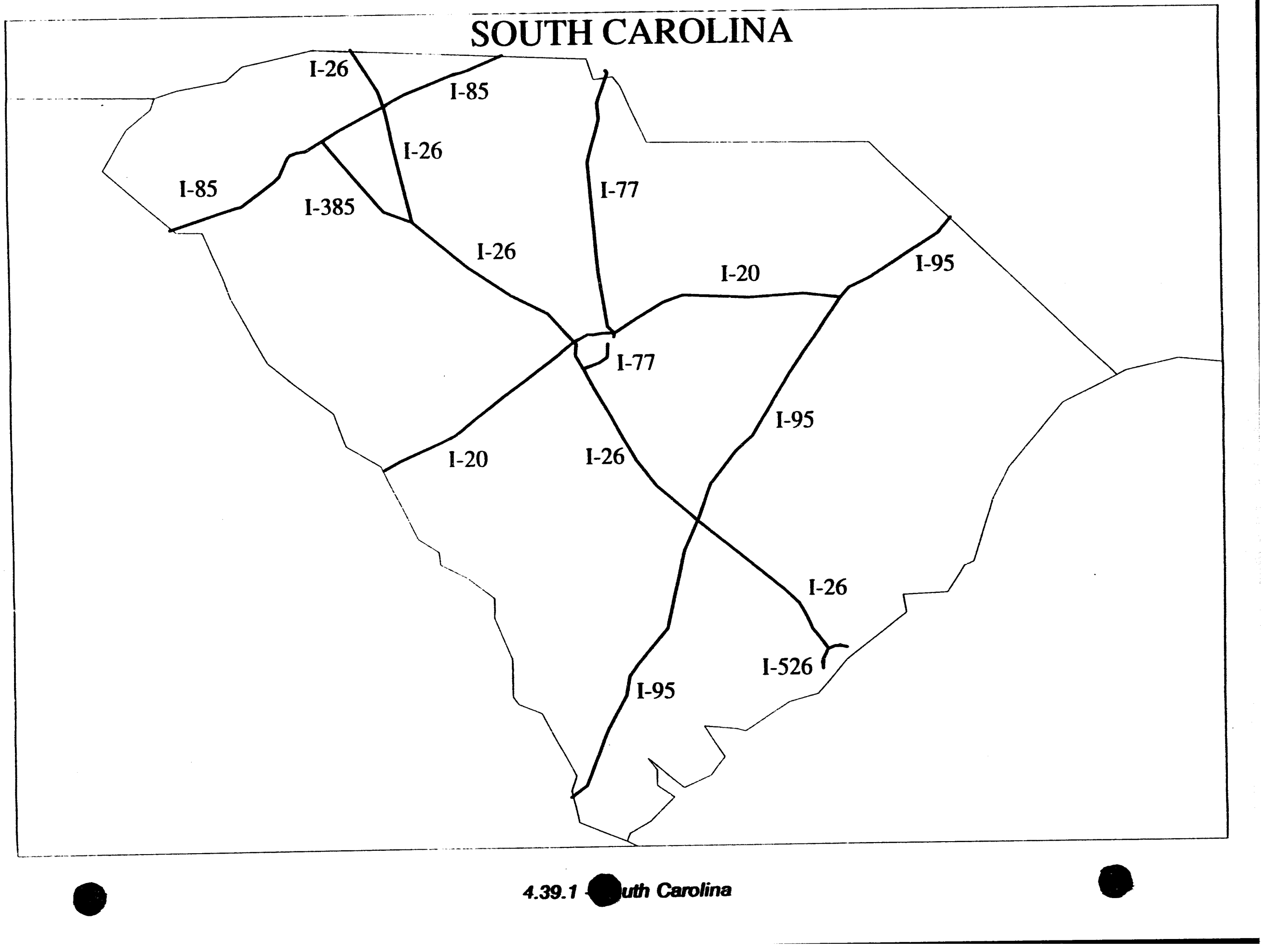




\section{Preferred Routes in South Dakota}

I-229 - From I-29 to I-90

I-29 From Nibraska state line to the North Dakota state line

I-90 From the Minnesota state line to the Wyoming state line

\section{State Designated Preferred Routes}

South Dakota has not designated any additional roads. 


\section{SOUTH DAKOTA}

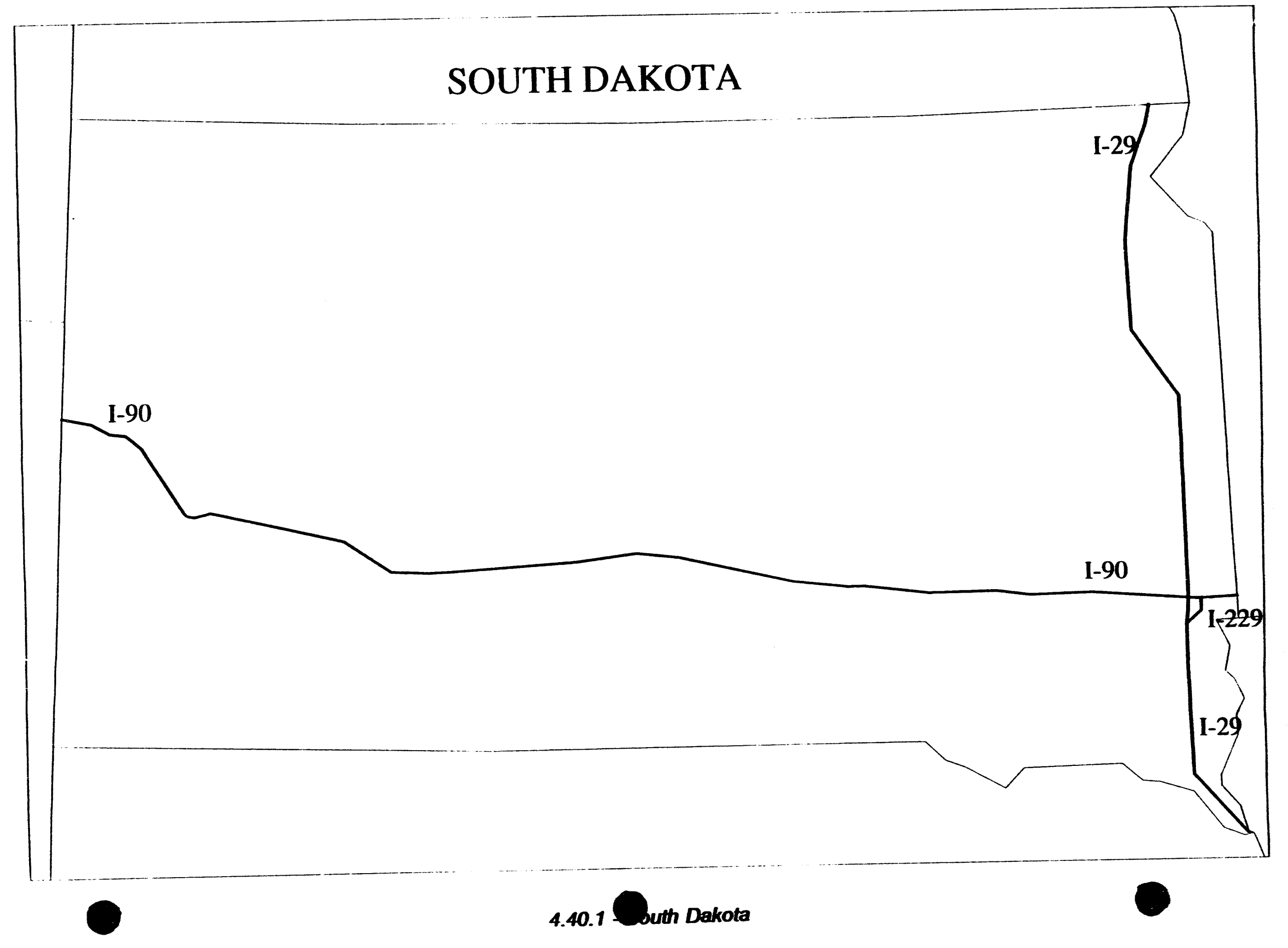




\section{Preferred Routes in Tennessee}

I-155 - From the Missouri state line to Dyershurg

I-181 - From Kingsport to Johnson City

1-24 From the Kentucky state line to 1-65 north of Nashville

- From $1-40$ in southeastern Nashville to the Georgia state line

- From the Georgia state line to I-75 east of Chattanooga

1-24/1-65 - From junction of 1-24 and 1-65 north of Nashville to 1-265 in Nashville

$1-240$ - From 1-40 east of Memphis to 1-55 south of Memphis

1-265 - From 1-40 to I-65 in Nashville

I-40 - From the Arkansas state line to I-265 in Nashville

- From 1-24 in southeastern Nashville to 1-75 west of Knoxville

- From 1-640 northeast of Knoxville to the North Carolina state line

1-40/1-75 - From junction of 1-40 and I-75 west of Knoxville to I-640 in Knoxville

1-440 - From I-40 to 1-24 in Nashville

1-55 - From the Arkansas state line to the Mississippi state line

1-640 - From 1-40/1-75 in Knoxville to 1-40 northeast of Knoxville

1-65 - From the Alabama state line to 1-440) in Nashville

- From I-24 north of Nashville to the Kentucky state line

1-75 - From the Georgia state line to I-40 west of Knoxville

- From I-640 in Knoxville to the Kentucky state line

$1-81$

- From the Virginia state line to I-40 near Dandridge

\section{State Designated Preferred Routes}

Tennessee has designated $1-640$ as a preferred route in lieu of $1-40$ in the Knoxville area. Per 49 CFR $177.825(\mathrm{~b})$, $1-640$ should be used instead of 1-40 because 1-640 is an Interstate System bypass around Knoxville. 


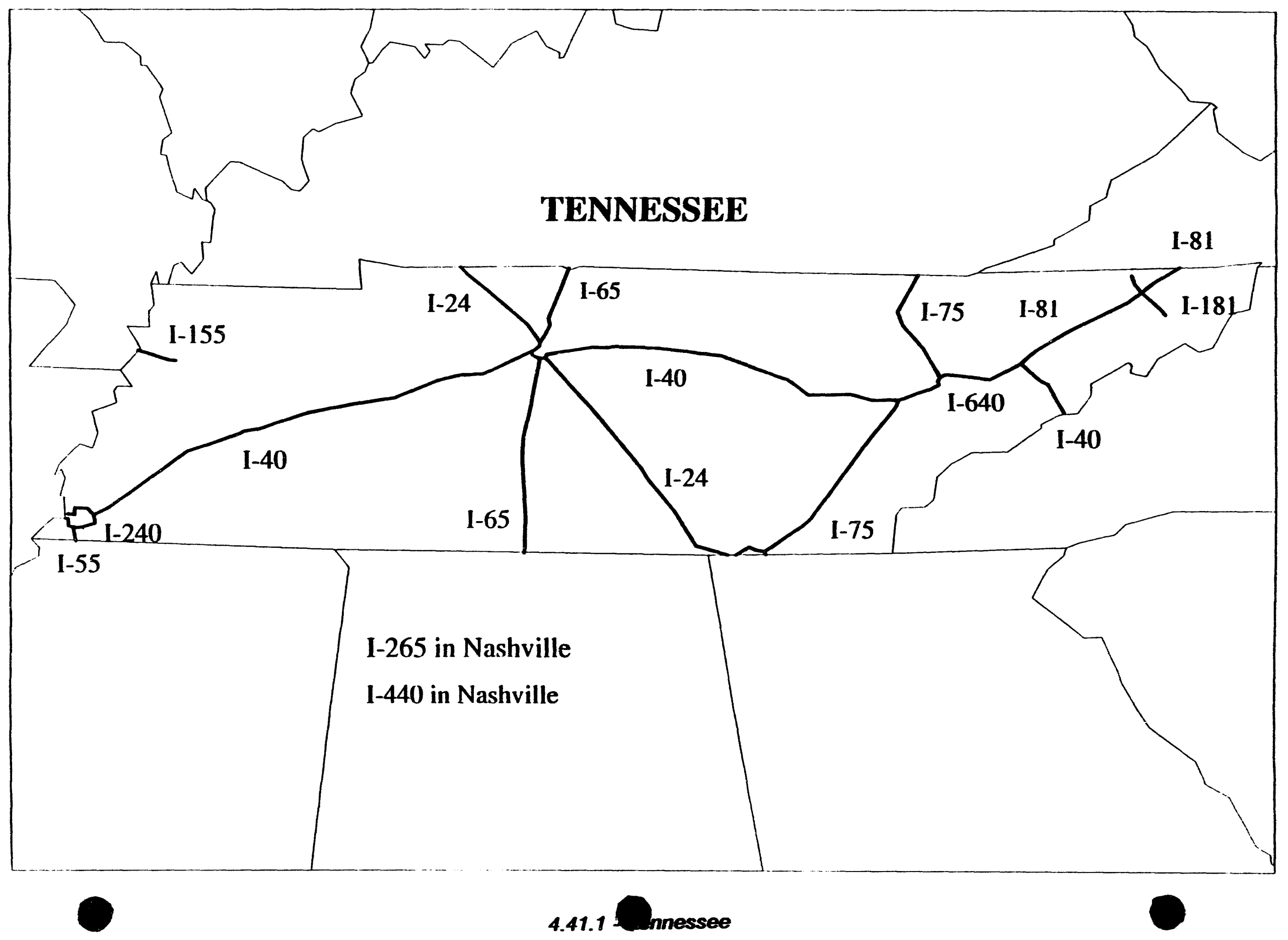





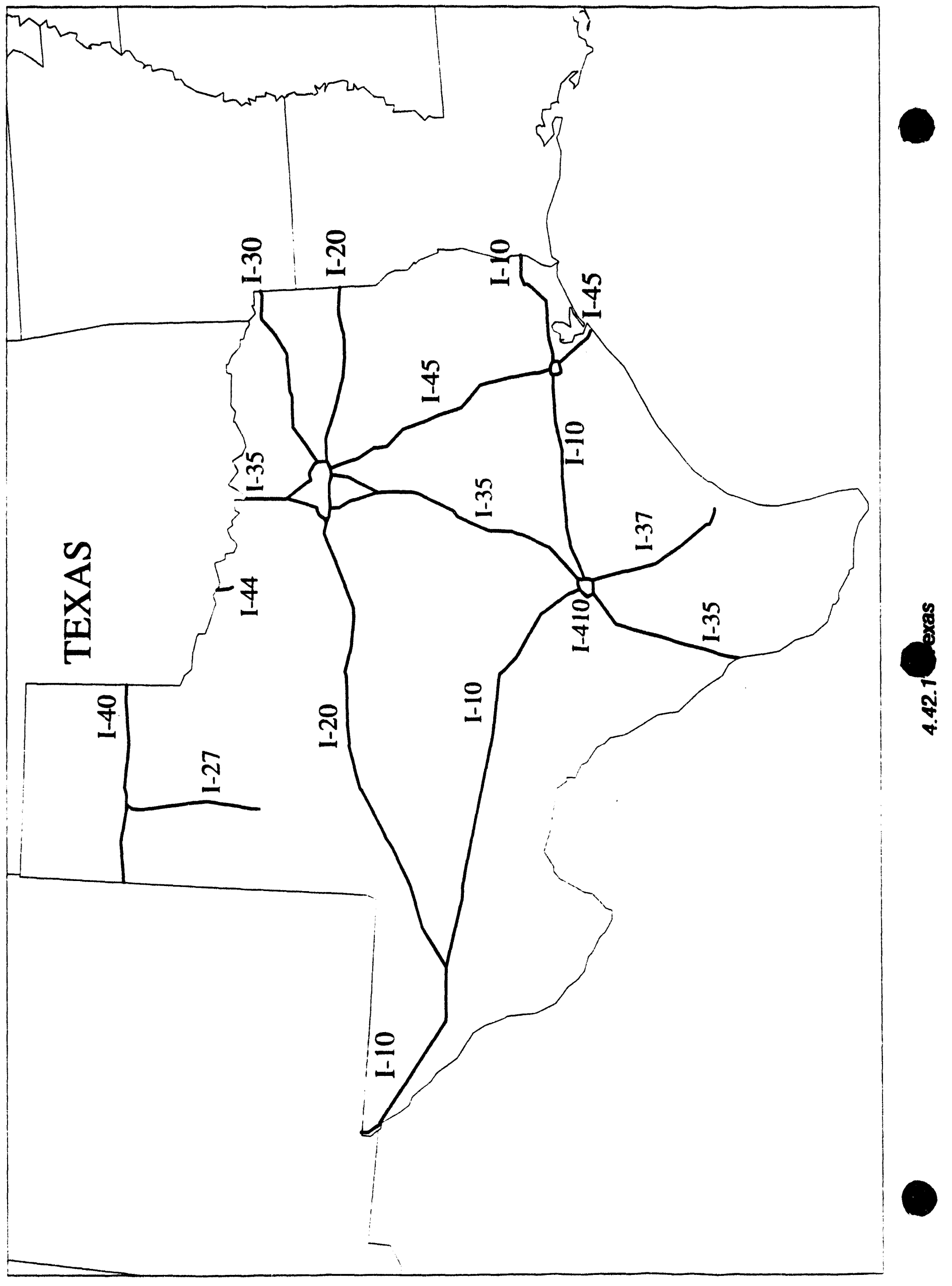




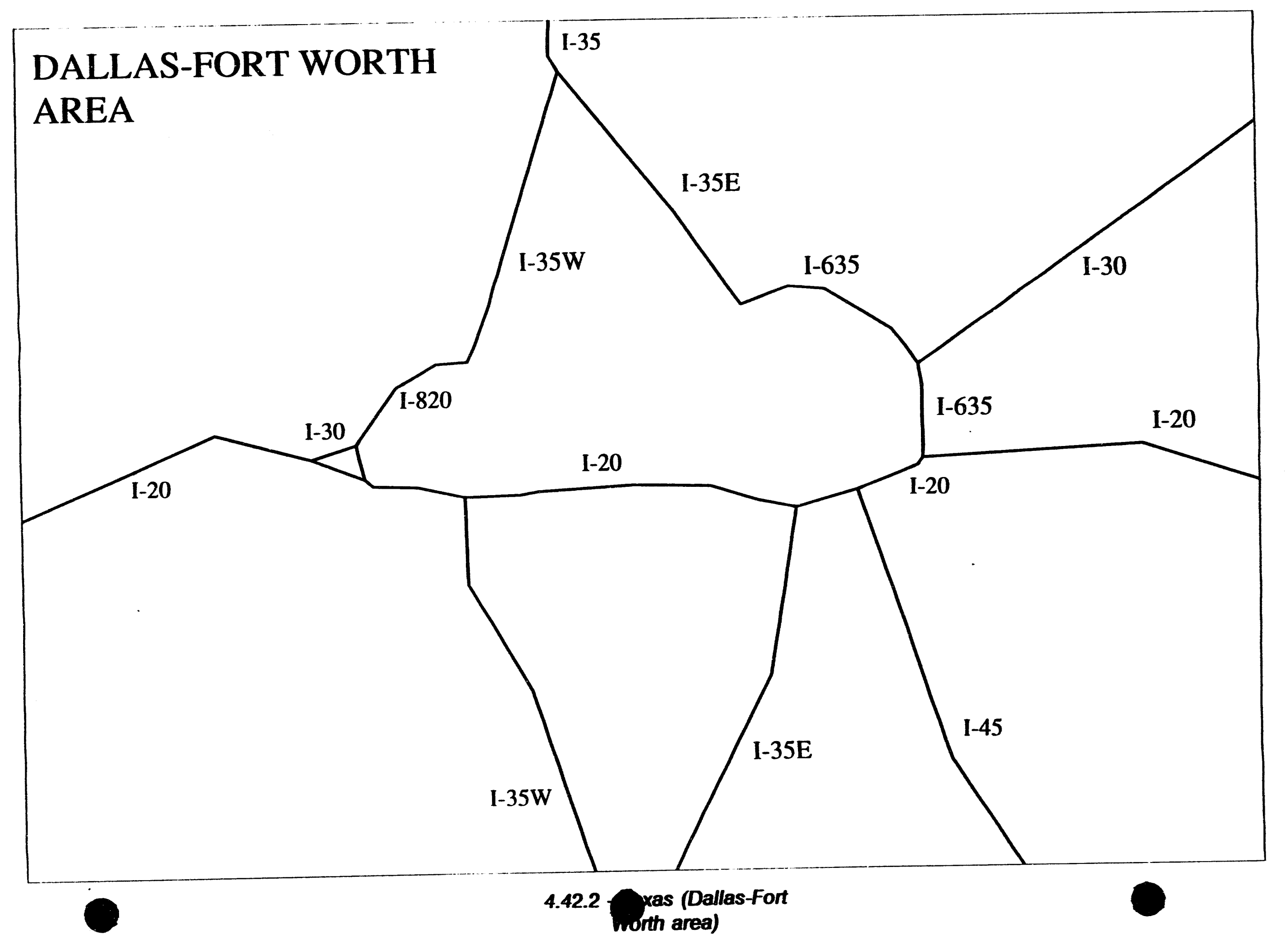




\section{Preferred Routes in Utah}

1-15 From the Arizona state line to 1-215 south of Salt Lake City

- From 1-215 north of Salt Lake City to the junction with I-84 near Ogden

- From the junction with 1-84 near Tremonton to the Idaho state line

I-15/I-84 - From junction of I-15 and I-84 near Ogden to junction of I-15 and I-84 at Tremonton

- From junction of 1-80 southeast of Salt Lake City to the junction with 1-15 north of Salt Lake City

I-70

- From I-15 to the Colorado state line

1-80 - From the Nevada state line to 1-215 west of Salt Lake City

- From junction of I-215 southeastern of Salt Lake City to the Wyoming state line

$1-84$

- From the Idaho state line to I-15 near Tremonton

- From junction with I-15 near Ogden to I-80

\section{State Designated Preferred Routes}

Utah has not designated any additional roads. 


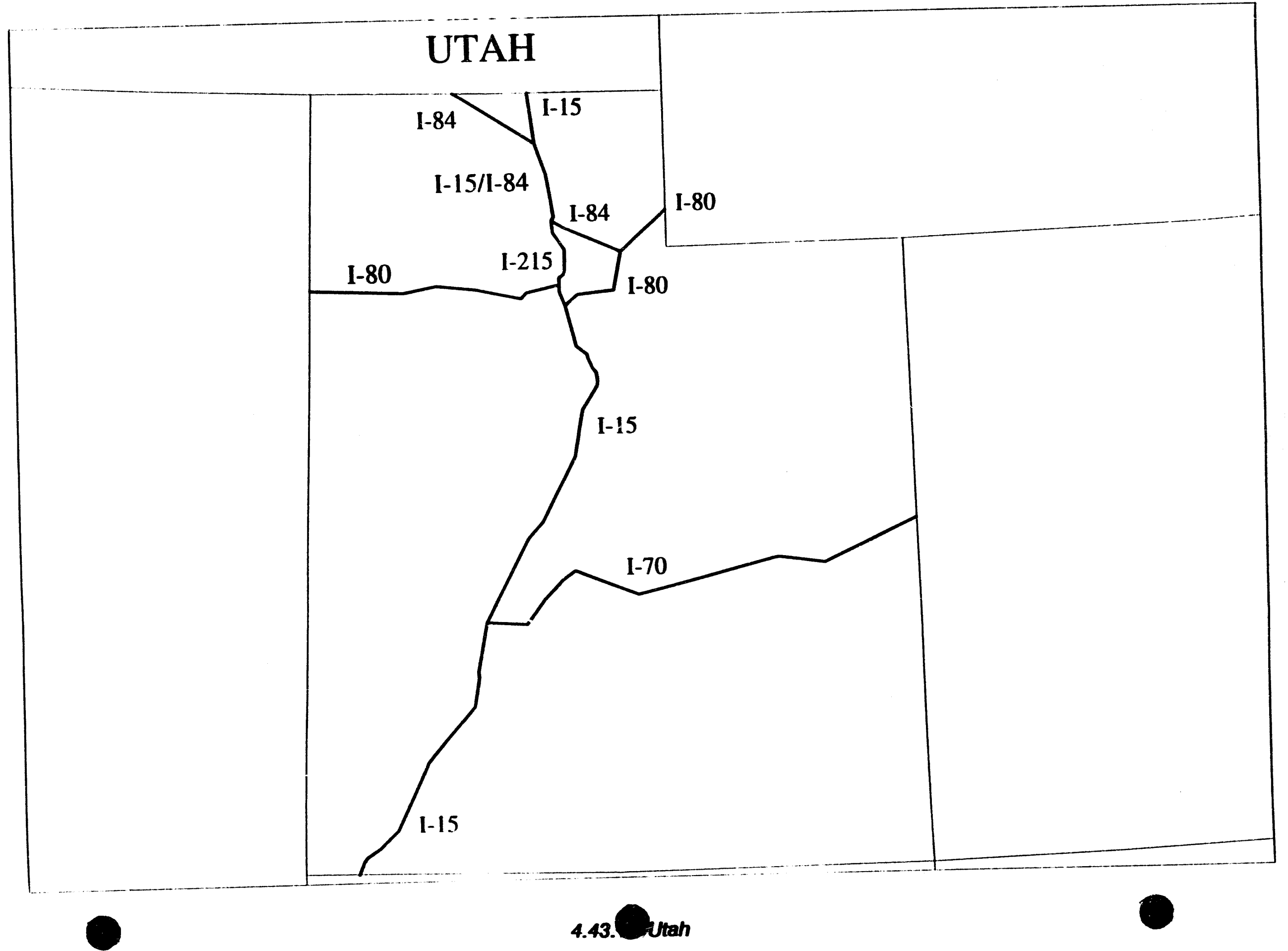



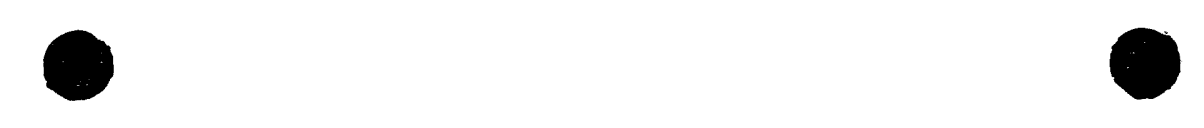

\section{Preferred Routes in Vermont}

1-89 From the New Hampshire state line to the Canadian border

I-91 - From Massachusetts state line to the Canadian border

1-93 From the New Hampshire state line to the junction with I-91 near St. Johnsbury

\section{State Designated Preferred Routes}

Vermont has not designated any additional roads. 


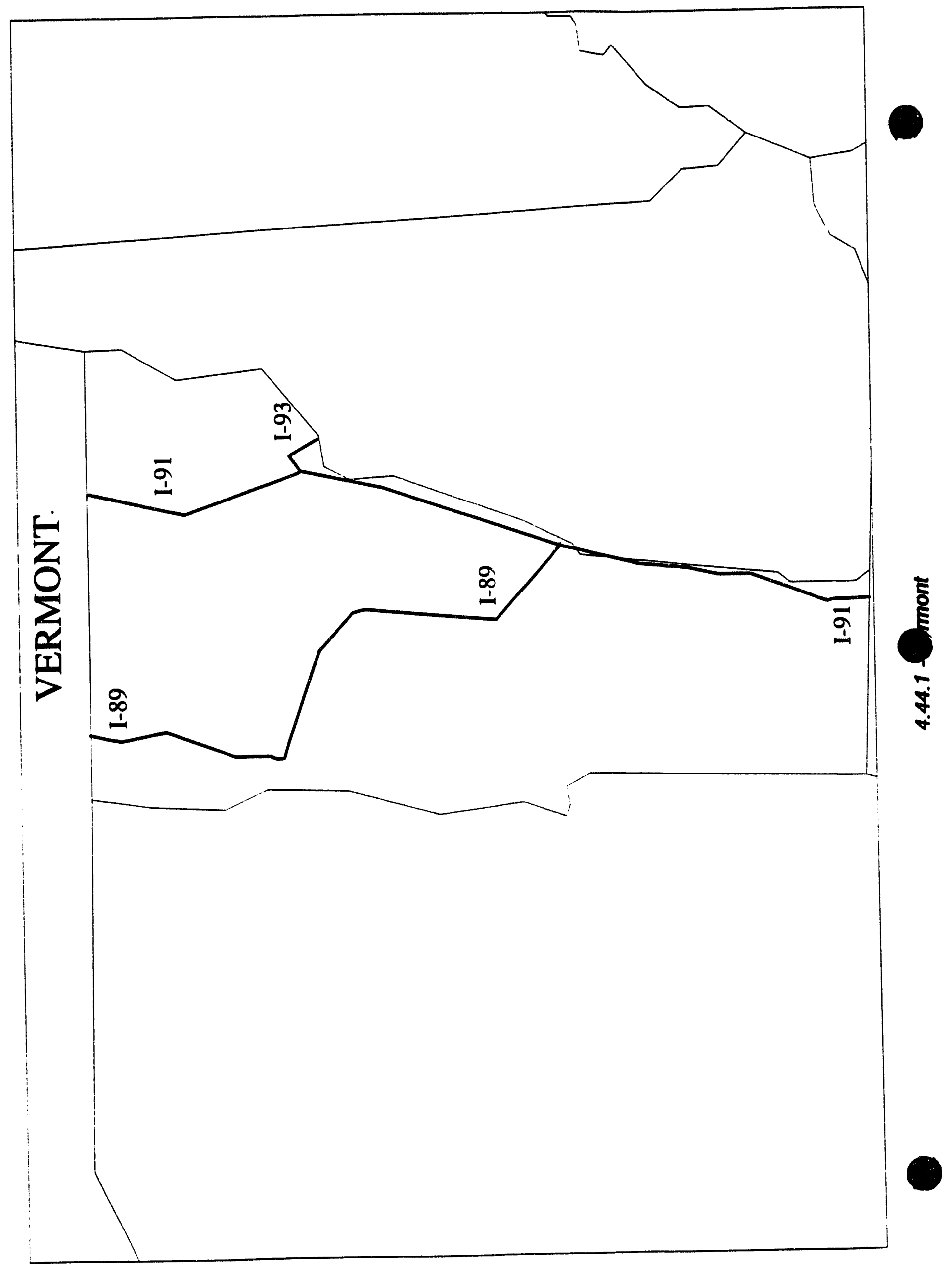




\section{Preferred Routes in Virginia}

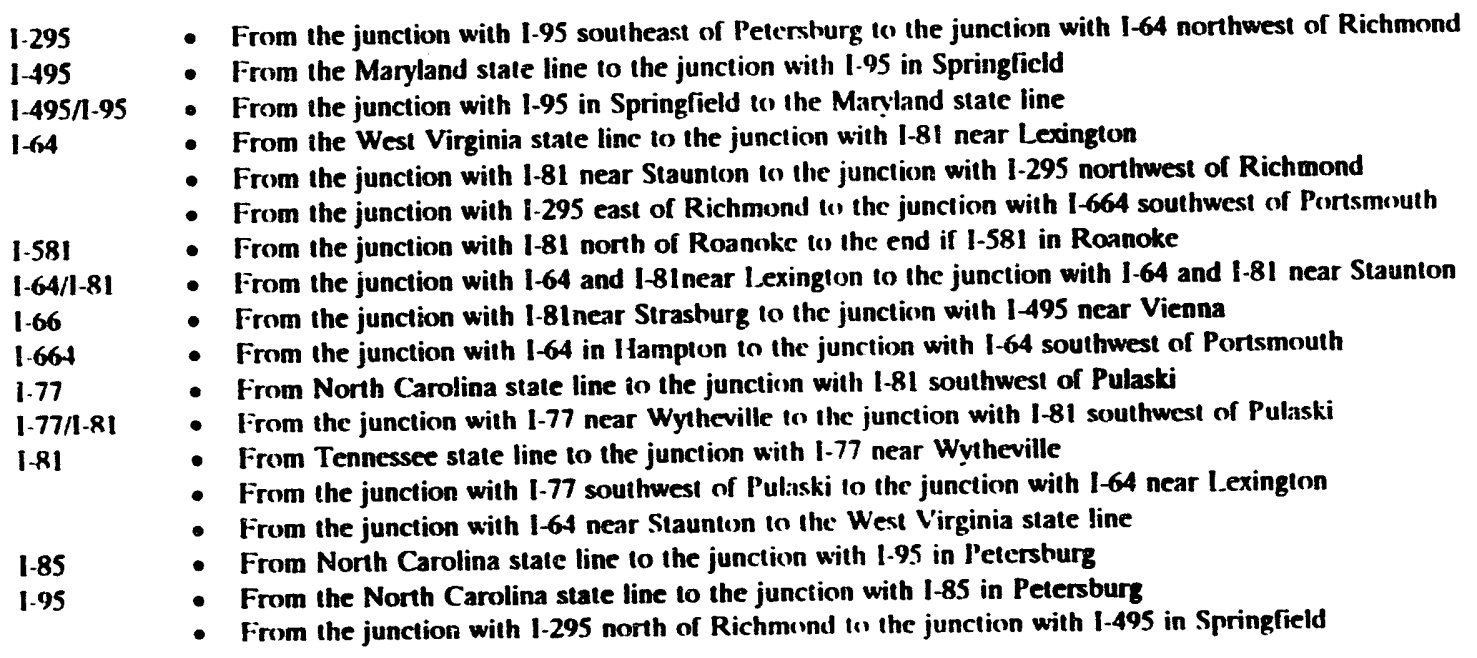

\section{State Designated Preferred Routes}

Virginia has designated the following additional roads. effective March 1. 1993:

\begin{tabular}{|c|c|}
\hline S.R. 207 & - Between 1-95 and U.S. 301 \\
\hline II.S. $30 !$ & - Between S.R. 207 and U.S. 17 \\
\hline II.S. 29 & - Between 1-66 and 1-64 \\
\hline II.S. 17 & - Between II.S. 301 and $1-81$ \\
\hline S.R. 208 & - Between I-95 and U.S. 522 \\
\hline II.S. 522 & - Between 1-64 and S.R. 208 \\
\hline S.R. 155 & - Between I-64 and S.R. 5 \\
\hline S.R. 5 & - Between S.R. 155 and S.R. 156 \\
\hline S.R. 156 & - Between S.R. 5 and S.R. 10 \\
\hline S.R. 10 & - Between S.R. 156 and U.S. 58 \\
\hline U.S. 4611 & - Between I.295 and II.S. 58 \\
\hline II.S. 58 & - Between 1-664 and 1-95 \\
\hline II.S. 460 & - Between 1-295 and U.S. 58 \\
\hline il.S. 258 & - Between 1-64 and S.R. IN \\
\hline (1.S. 460 & - Between 1-581 and 1-85. \\
\hline (I.S. 460 & - Between the West Virginia state line and S.R. I(W) \\
\hline $\begin{array}{l}\text { S.R. I(M) } \\
1.77\end{array}$ & $\begin{array}{l}\text { - Between } I-81 \text { and U.S. } 461) \\
\text { - Banned helween } I-81 \text { and the West } V\end{array}$ \\
\hline .77 & - Banned hetween 1-81 and the West Virginia stalc li \\
\hline
\end{tabular}




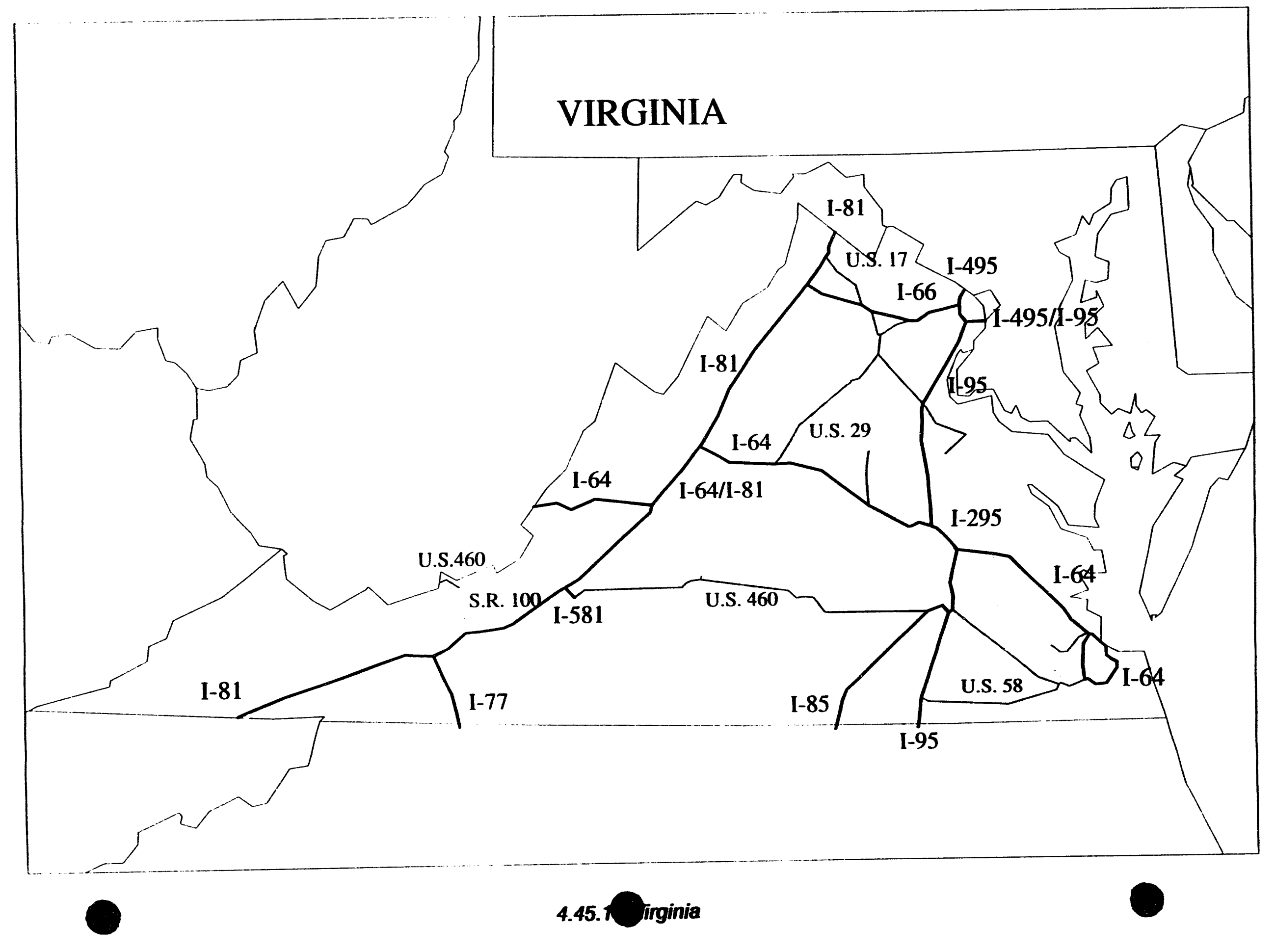




\section{Preferred Routes in Washington}

I-182 From I-82 to Pasco

I-205 From the Oregon state line to I-5 north of Vancouver

1-405 - From junction of 1-5 south of Seattle to junction of I-5 north of Seattle

1-5 From the junction with 1-205 north of Vancouver to junction with 1-405 south of Seattle

- From junction with 1-405 north of Seattle to the Canadian border

1-82 From Oregon state line to 1-90

1-90 - From I-405 to Idaho state line

\section{State Designated Preferred Routes}

Washington has not designated any additional roads. 


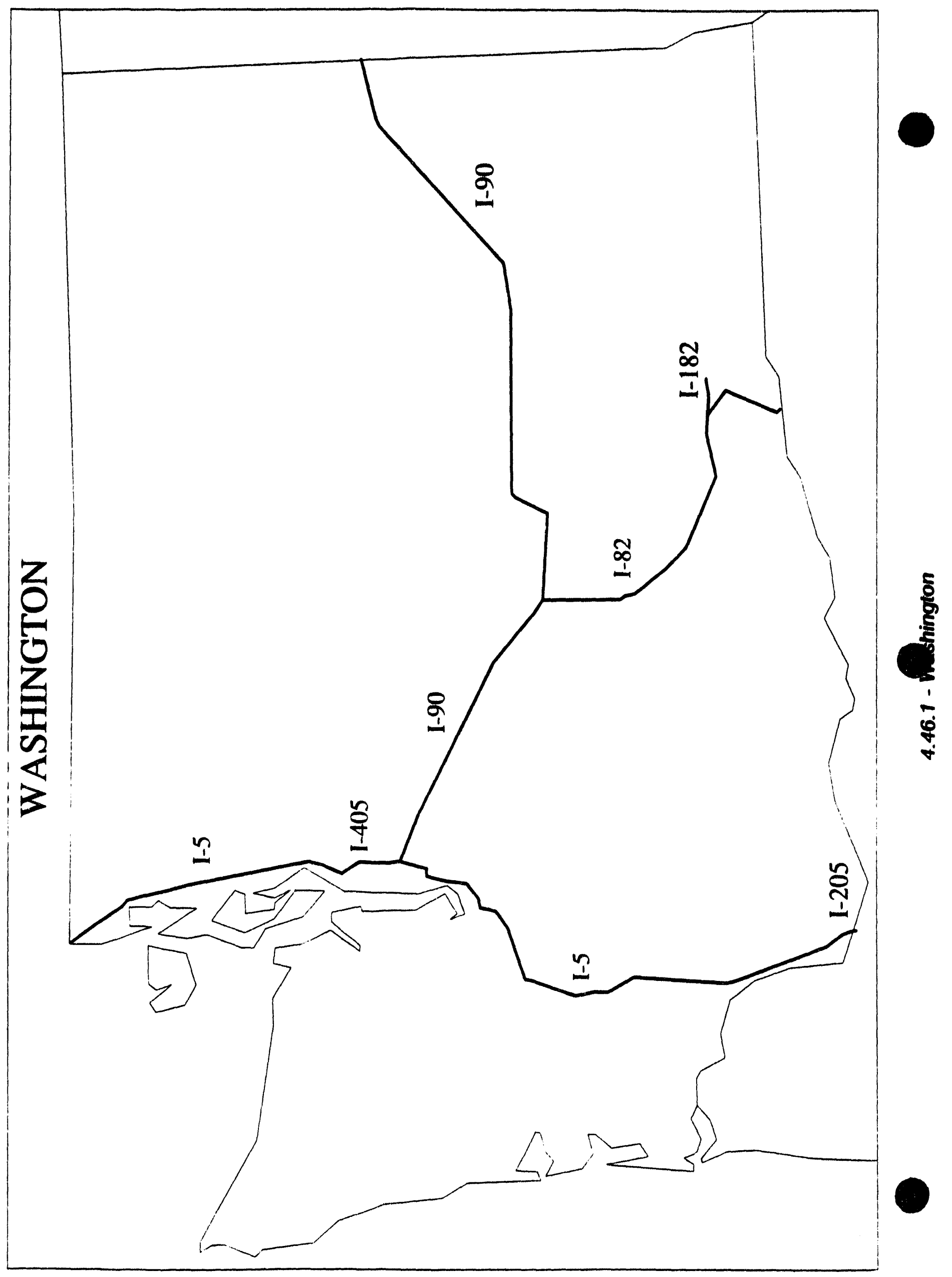




\section{Preferred Routes in West Virginia}

$1-470$

- From the Ohio state line to the junction with I-70 in Wheeling

1-64 From the Kentucky state line to the junction with 1-77 in Charleston

- From the junction with I-77 south of Beckley to the Virginia state line

I-64/I-77 - From the junction of I-64 and I-77 in Charleston to the junction of I-64 and I-77 south of Beckley

I-68 From the junction with 1-79 south of Morgantown to the Maryland state line

1-70 - From the junction with 1-470 in Wheeling to the Pennsylvania state line

1-77 From the junction with U.S. 52 east of Bluefield to the junction with 1-64 south of Beckley

- From the junction with 1-64 in Charleston to the Ohio state line

I-79 - From the junction with I-77 north of Charleston to the Pennsylvania state line

1-81 - Frem the Virginia state line to the Maryland state line

\section{State Designated Preferred Routes}

West Virginia has not designated any additional roads, but shipments coming from Virginia are banned from using $1-77$ (due to tunnels in Virginia). Virginia has designated U.S. 460 as the port of entry into West Virginia. 


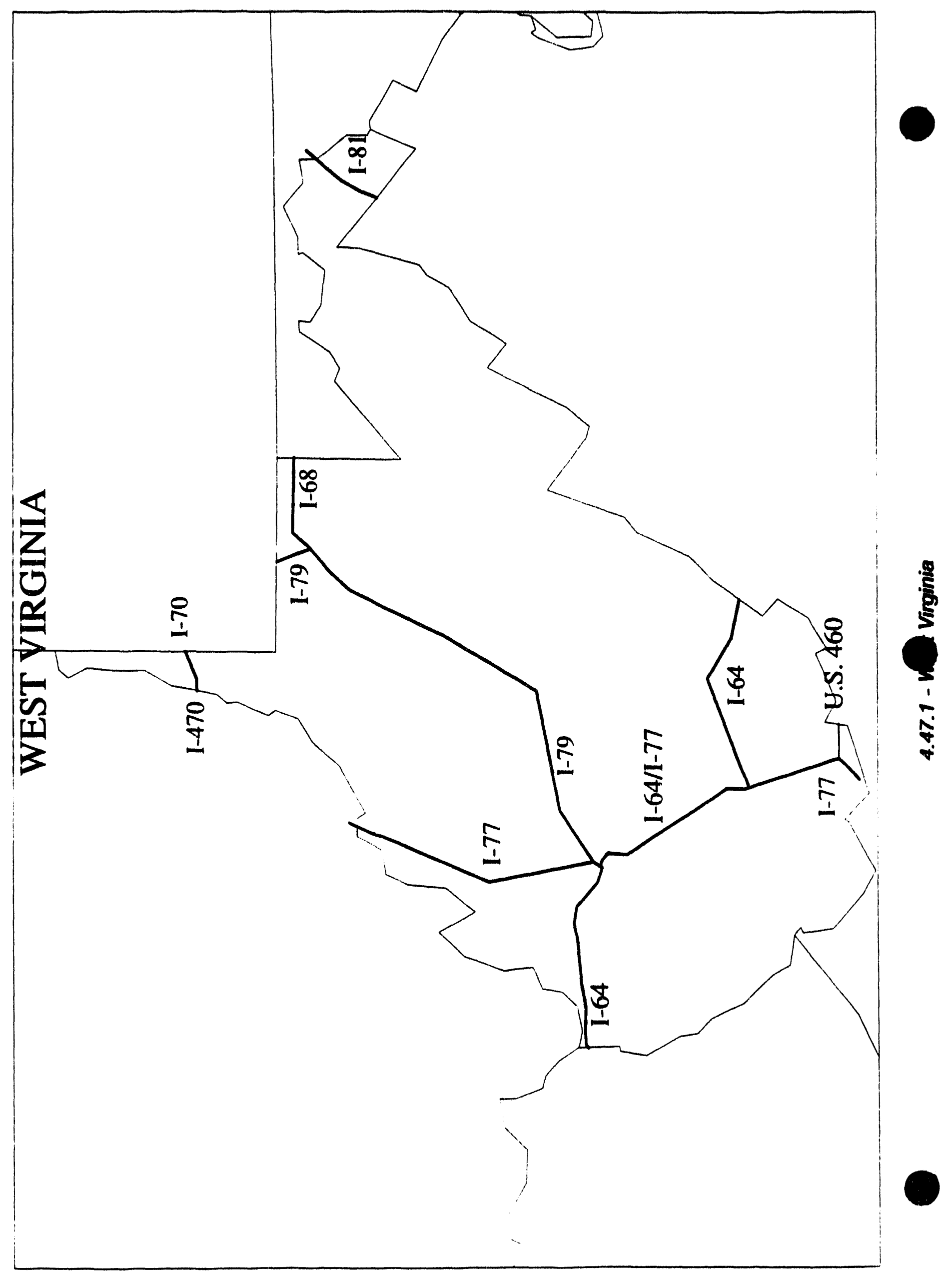




\section{Preferred Routes in Wisconsin}

1-43 From the junction with 1-90 east of Beloit to the junction with 1-894 southwest of Milwaukee

- From the junction with I-94 in Milwaukee to Green Bay

1-43/I-894 - From the junction of 1-43 and I-894 southwest of Milwaukee to the junction of I-94 south of Milwaukee

1-43/1-94 - From the junction of 1-43/I-894/I-94 south of Milwaukee to the junction with I-794 in Milwaukee

$1-535$

- From the Minnesota state line to Superior

I-894

- From junction of I-94 west of Milwaukee to junction of I-43 southwest of Milwaukee

1-90 - From the Minnesota state line to junction with 1-94 near Tomah

- From junction with 1-94 east of Madison to the Illinois state line

I-90/I-94 - From the junction of I-90 and 1-94 near Tomah to the junction of 1-90 and I-94 east of Madison

I-94 From the Minnesota state line to the junction with 1-90 near Tomah

- From the junction with 1-90 east of Madison to the junction with 1-43 in Milwaukee

- From the junction with 1-43 and 1-894 south of Milwaukee to the Illinois state line

\section{State Designated Preferred Routes}

Wisconsin has not designated any additional roads. 


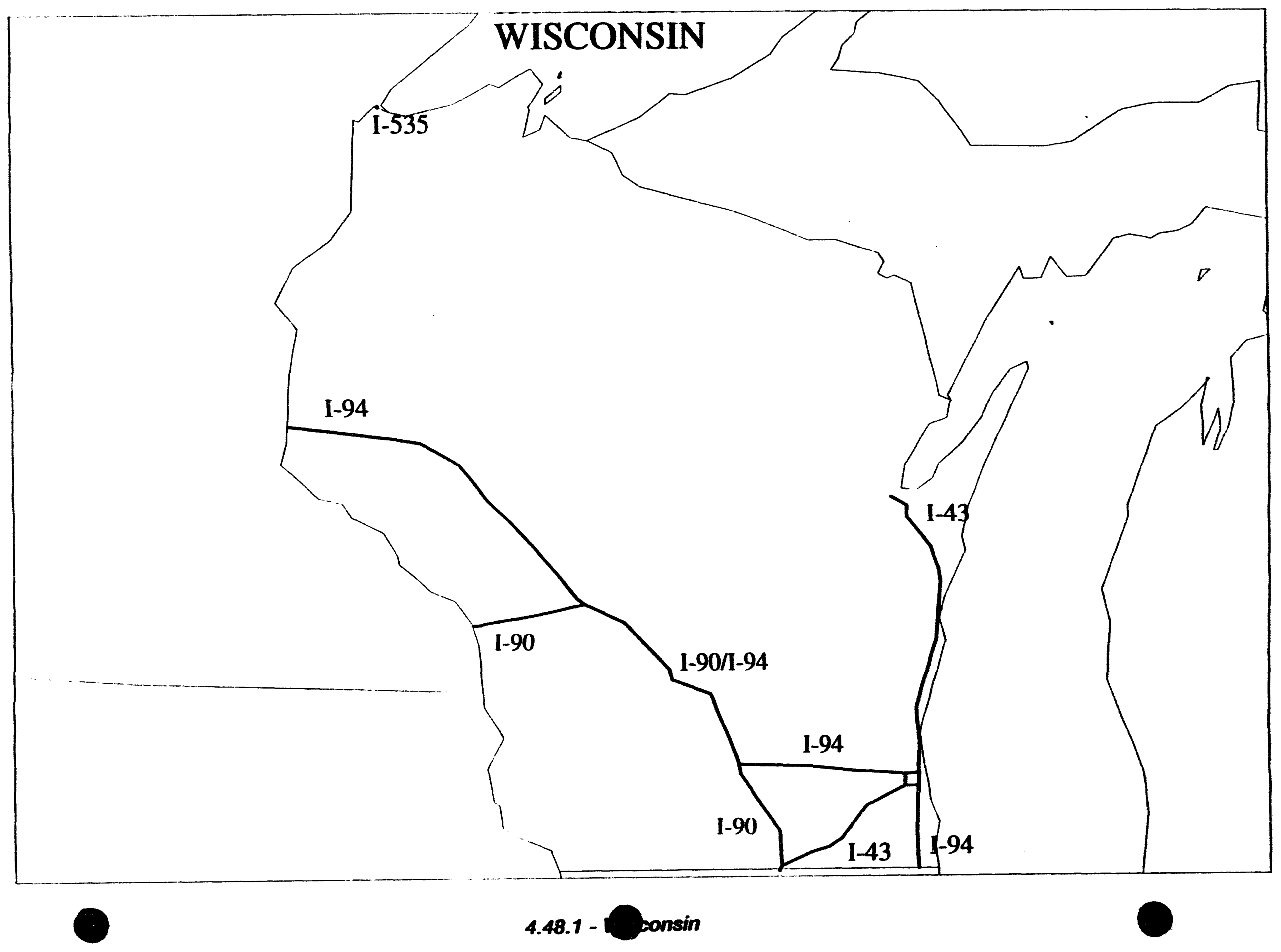



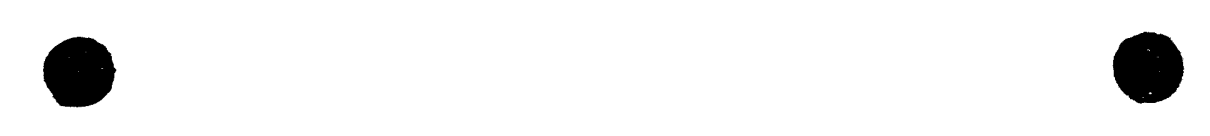

\section{Preferred Routes in Wyoming}

1-25 From the Colorado state line to 1-90

1-80 - From the Nebraska state line to the Utah state line

1-90 - From the Montana state line to the South Dakota state line

\section{State Designated Preferred Routes}

Wyoming has not designated any additional roads. 


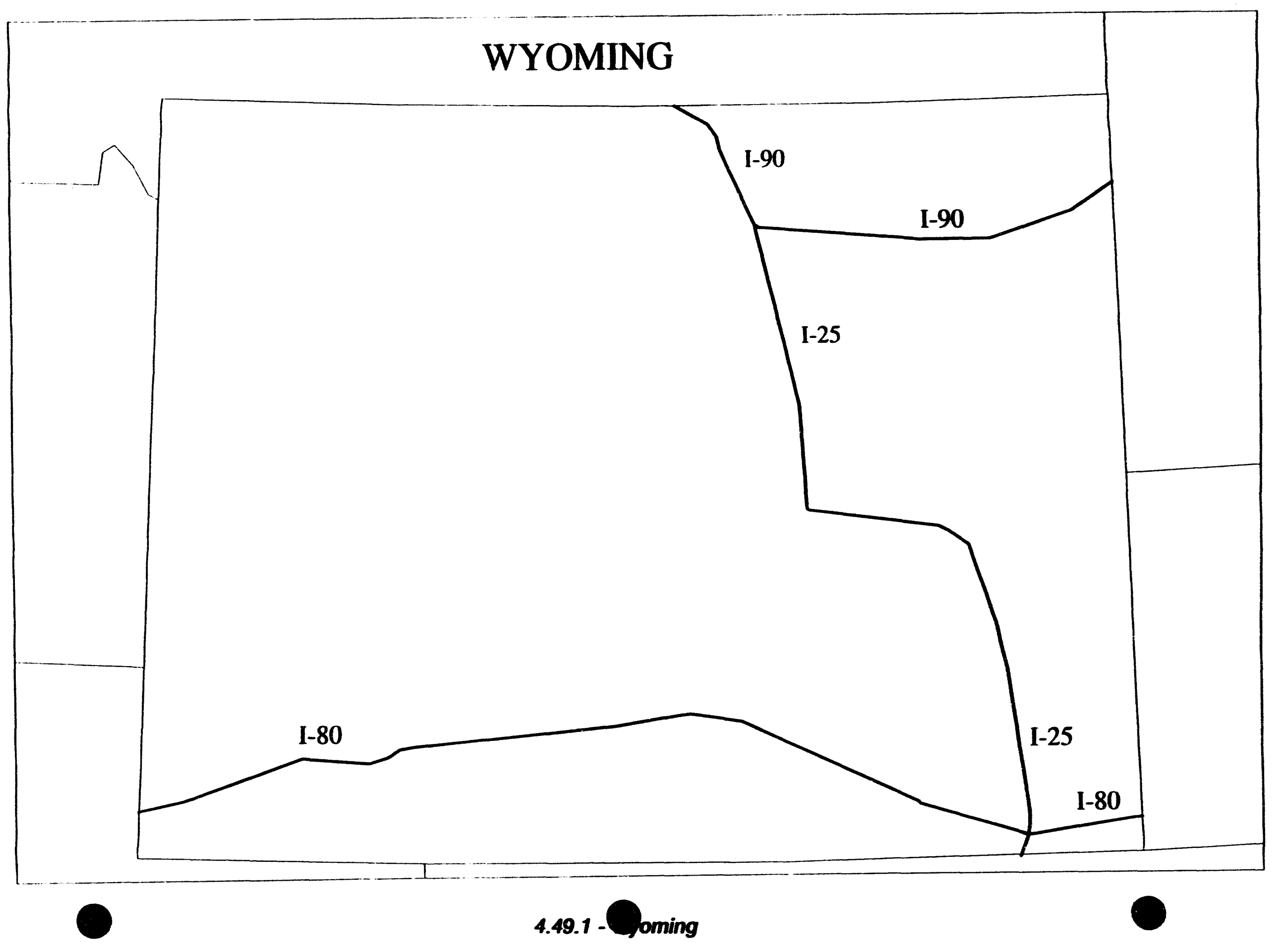




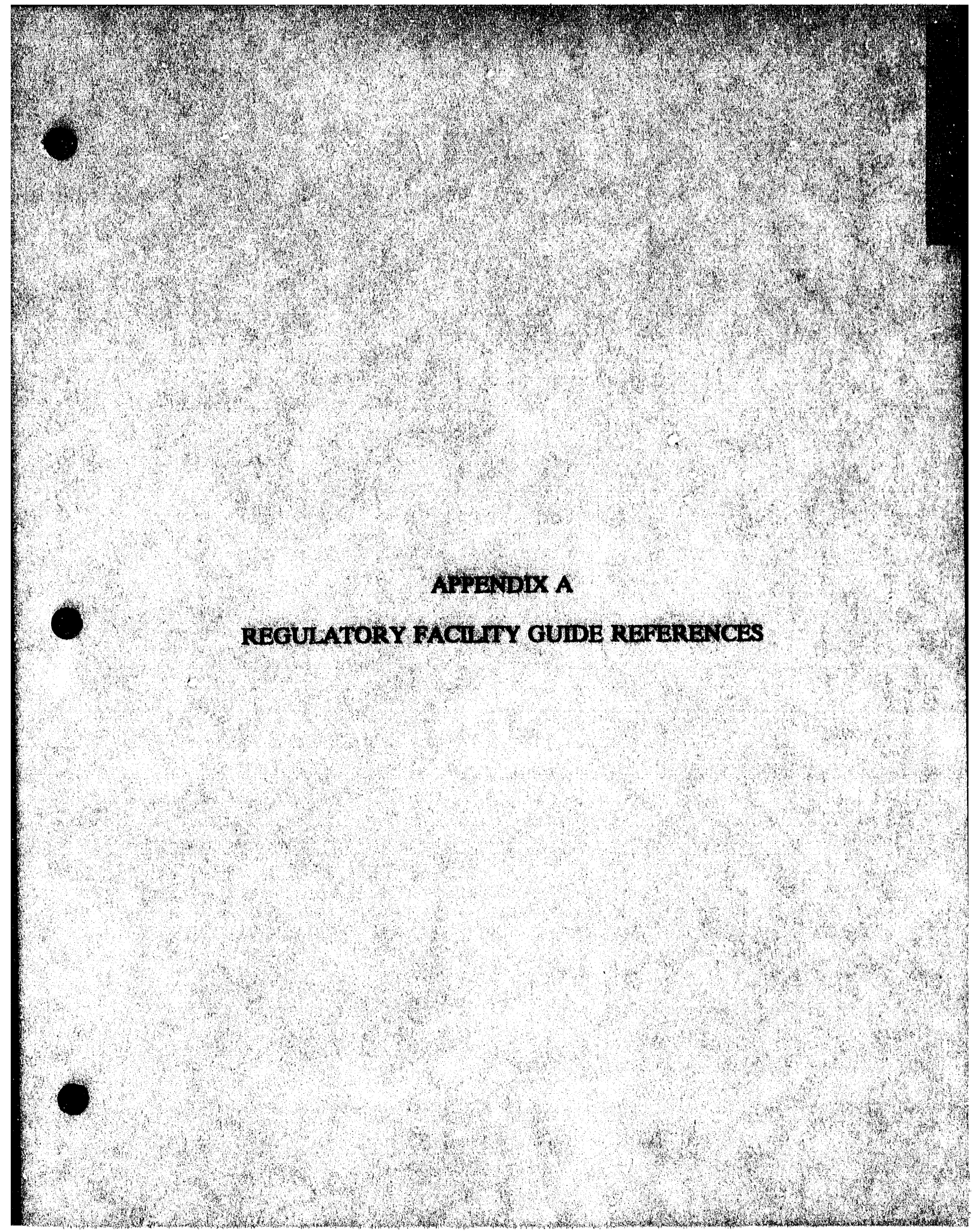




\section{APPENDIX A \\ Regulatory Facility Guide References}

'Code of Federal Regulations (CFR), 10 CFR - Energy, Government Printing Office, Washington, January 1993.

'Code of Federal Regulations (CFR), 15 CFR - Commerce and Foreign Trade, Government Printing Office, Washington, January 1993.

'Code of Federal Regulations (CFR), 19 CFR - Articles Exported and Retumed, Government Printing Office, Washington, April 1993.

'Code of Federal Regulations (CFR), 29 CFR - Labor, Government Printing Office, Washington, July 1992.

'Code of Federal Regulations (CFR), 40 CFR - Protection of Environment, July 1992.

'Code of Federal Regulations (CFR), 49 CFR - Transportation, Government Printing Office, Washington, October 1992.

International Air Transport Association (IATA), Dangerous Goods Regulations, 35th Edition, Effective January 1, 1994, Montreal, Quebec, 1994.

'International Maritime Operations (IMO), International Maritime Dangerous Goods Code, Vols. 1-4, London, 1991.

${ }^{3}$ J.J. Keller \& Associates, Inc., Hazardous Waste Regulatory Guide: State Waste Management Programs, Generator, Transponer, Treatment, Storage \& Disposal Standards, Neenah, WI, 1993.

${ }^{3}$ J.J. Keller \& Associates, Inc., 1910 OSHA Guide: Plant Safety Regulations \& Index, Neenah, WI, 1991.

${ }^{3}$ J.J. Keller \& Associates, Inc., Hazardous Waste Guide: For Generators, Transporters \& TSD's, Neenah, WI, 1992.

${ }^{3}$ J.J. Keller \& Associates, Inc., Fleet Safety Compliance Manual: Procedures, Regulations, Source Data: Personnel, Operations and Equipment, Neenah, WI, 1992.

${ }^{3} \mathrm{~J} . J$. Keller \& Associates, Inc., Hazardous Waste Operations \& Emergency Response Compliance: OSHA Part 1910.120, Neenah, WI, 1992. 
${ }^{3}$ J. J. Keller \& Associates, Inc., Motor Carrier Safety Report, Volume XIX, 1993 (published monthly).

${ }^{3}$ J.J. Keller \& Associates, Inc., Vehicle Sizes \& Weights Manual, Neenah, WI, 1993.

${ }^{3}$ J.J. Keller \& Associates, Inc., Trucking Safety Guide, Neenah, WI, 1993.

${ }^{3}$ J.J. Keller \& Associates, Inc., Hazardous Materials 181: The Guide for Shippers, Handlers \& Transporters, Neenah, WI, 1993.

${ }^{3}$ J.J. Keller \& Associates, Inc., State Hazardous Materials Manual: State Requirements for Shipping and Transportation, Neenah, WI, 1993.

${ }^{3}$ J.J. Keller \& Associates, Inc., Hazardous Waste Management Guide: Identification, Monitoring, Treatment and Disposal. Regulations, Compliance \& Management, Neenah, WI, 1993.

${ }^{3}$ J.J. Keller \& Associates, Inc., Trucking Permit Guide: Private, Contract, Common, Exempt, Neenah, WI, 1993.

${ }^{3}$ J.J. Keller \& Associates, Inc., Drug Testing: Motor Carrier Compliance Manual, Neenah, WI, 1993.

W. E. Kenworthy, Transportation of Hazardous Materials, Second Edition, $A$ Compliance and Practice Guide for Safe Transportation of Hazardous Materials, Government Institutes, Inc., 1992.

${ }^{4}$ Office of Executive Director, Conference of Radiation Control Program Directors, Inc., Directory of State Agencies Involved with the Transportation of Radioactive Material - With Notes on Their Statutory Authority and Regulations, CRCPD Publication 92-6, Frankfort, KY, November 1993.

${ }^{5}$ Tennessee Code Annotated, Section 110, Liability Insurance Requirements, 1982.

5Tennessee Code Annotated, Section 113, Safety Rules and Regulations Inspection of Vehicles..., 1991 Supplement. 
Ordering information for footnoted references:

'C.W. Associates

P.O. Box 34099

Bethesda, MD 20827

Telephone: (301) 340-9399

'American Labelmark Company

Labelmaster Division

5724 North Pulaski Road

Chicago, IL 60646-6797

Telephone: (312) 478-0900; Fax: (312) 478-6054

${ }^{3} \mathrm{~J} . J$. Keller \& Associates, Inc.

$3003 \mathrm{~W}$. Breezewood Lane

P.O. Box 368

Neenah, WI 54957-0368

${ }^{4}$ Executive Director

Conference of Radiation Control Program Directors, Inc.

205 Capital Avenue

Frankfort, KY 40601

Telephone: (502) 227-4543

${ }^{5}$ Tennessee Motor Vehicle Laws Annotated (Reprinted from the Tennessee Code Annotated)

Nashville, TN 37243-0505

Telephone: (615) 741-2974, 1-800-342-8359 


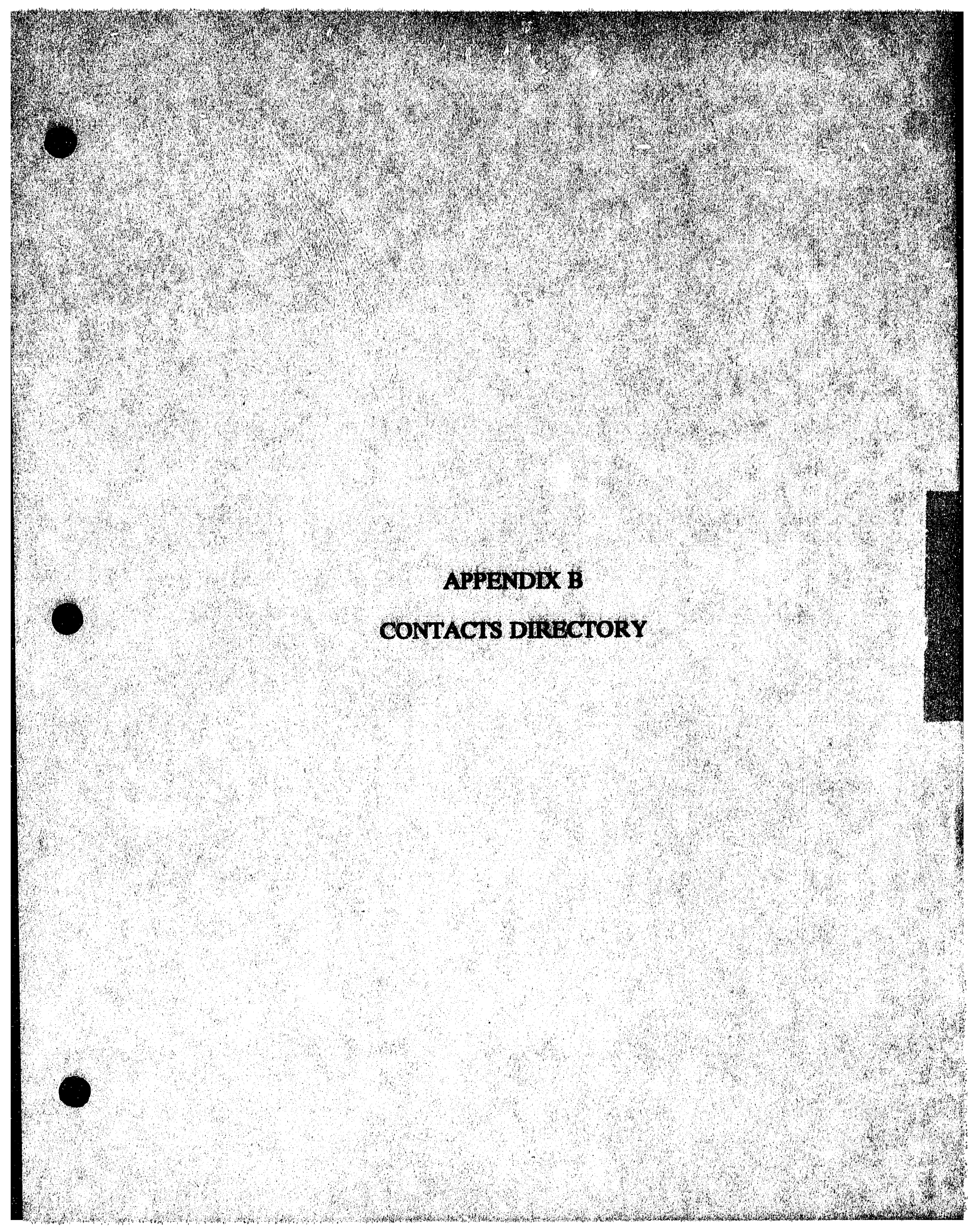




\title{
APPENDIX B \\ CONTACTS DIRECTORY
}

\author{
Analysas Corporation \\ Oak Ridge Corporate Center \\ 151 Lafayette Drive \\ Oak Ridge, TN 37830
}

Contact: Barbara Turner. Manager, Transportation Operations

Telephone: (615) 576-9545; FAX: (615) 576-0709

Contact: Mike Bohlen, Manager, Packaging Operations

Telephone: (615) 576-2245; FAX: (615) 576-9417

Bechtel National, Inc.

P.O. Box 350

Oak Ridge, TN 37831

Contact: Karen Noey, Supervisor. FUSRAP Waste Management

Telephone: (615) 576-4432: FAX: (615) 576-2249

Department of Energy

Oak Ridge Operations

P.O. Box 2001

Oak Ridge, TN 37831

Contact: Brady Lester, Transportation Manager

Telephone: (615) 576-8354; FAX: (615) 576-5333

Contact: Marvin Bennett, Packaging Manager

Telephone: (615) 576-0853; FAX: (615) 576-5333

Cuntact: Doug Stancel, Transportation Safety

Telephone: (615) 576-9214; FAX: (615) 576-3725

Contact: Dana Willaford, Transportation Safety

Telephone: (615) 576-5338; FAX: (615) 576-3725

Martin Marietta Energy Systems, Inc.

P.O. Box 2009

Oak Ridge, TN 37831-8241

Cuntact: Brad Welles, Transportation Compliance Manager

Telephone: (615) 574-6376; FAX: (615) 241-2023 
Martin Marietta Energy Systems, Inc.

P.O. Box 2008

Oak Ridge, TN 37831-6267

Contact: Randy Walker. Director of Packaging \& Transportation

Telephone: (615) 574-5522: FAX: (615) 574-1979

Martin Marietta Energy Systems, Inc.

Oak Ridge National Laboratory

P.O. Box 2008

Oak Ridge, TN 37831-6267

Contact: Randy Walker, Transportation Operations

Telephone: (615) 574-5522; FAX: (615) 574-1979

Contact: Mark Hawk. Packaging Operations

Telephone: (615) 574-6042; FAX: (615) 574-1979

Martin Marietta Energy Systems, Inc.

Y-12 Site

P.O. Box 2009

Oak Ridge, TN 37831-8043

Contact: Roger Yarborough, Transportation Operations

Telephone: (615) 574-6550; FAX: (615) 574-8612

Contact: Ed Stumpfl, Packaging Operations

Telephone: (615) 574-2547; FAX: (615) 574-3514

Martin Marietta Energy Systems, Inc.

K-25 Site

P.o. Box 2003

Oak Ridge, TN 37831-7532

Contact: Henry Nennstiel. Transportation Operations

Telephone: (615) 574-4049; FAX: (615) 576-2838

Contact: Jerry Rutherford. Packaging Operations

Telephone: (615) 574-8950; FAX: (615) 574-1979

MK-Ferguson of Oak Ridge Company

P.O. Box 2011

Oak Ridge, TN 37831-2011

Contact: Judy Hedden. Transportation Operations

Telephone: (615) 574-3033 
Oak Ridge Institute for Science \& Education

P.O. Box 117

Oak Ridge, TN 37831-0117

Contact: Larry O'Barr, Asst. Director, Office of Facilities Management. Transportation. \& Svcs.

Telephone: (615) 576-9246: FAX: (615) 576-7046

Science Applications International Corporation

301 Laboratory Rd.

Oak Ridge, TN 37831

Contact: Cheryl Hamberger, SMAC Coordinator

Telephone: (615) 481-2913; FAX: (615) 482-6828

Contact: Steve Schmid. Packaging Operations

Telephone: (615) 481-2899; FAX: (615) 481-8591

Tennessee Emergency Management Agency

3041 Sidco Dr.

Nashville. TN 37204

Contact: John White, Asst. Deputy Director

Telephone: (615) 741-0001

AFTER HOURS: (Inside TN - 1-800-262-3300) (Outside TN - 1-800-258-3300)

(Govemor Designee for Receiving Advance Notification of Transportation of Nuclear Waste Shipments)

Tennessee Public Service Commission

460 James Robertson Parkway

Nashville. TN 37243-0505

Contact: Paul Melander, Manager, Motor Carrier Investigations

Telephone: (615) 741-2974: 1-800-342-8359

Tennessee Department of Environmental and Conservation

Division of Radiological Health

401 Church St., 3rd Floor, L\&C Annex

Nashville, TN 37243-1532

Contact: Elizabeth Flanagan, Emergency Services Coordinator

Telephone: (615) 532-0416

(Radioactive Materials Licensing, Emergency Response)

Tennessee Department of Environment and Conservation

Division of Solid Waste Management

401 Church St., 5th Floor, L\&C Annex

Nashville, TN 37243-1535

Contact: Alcyone Arnold

Telephone: (615) 741-3424 
Tennessee Department of Safety

1150 Foster Ave.

Hardison Building

Nashville. TN 37249

Contact: Burton Lawson

Telephone: (615) 251-5196

(Enforcement Agency for Vehicle Sizes \& Weights)

Tennessee Department of Transportation

Overweight \& Overdimensional Permit Section

Suite 300, James K. Polk Bldg.

505 Deaderick St.

Nashville, TN 37243-0331

Contact: Jerry W. Smith, Civil Engineer

Telephone: (615) 741-3821 


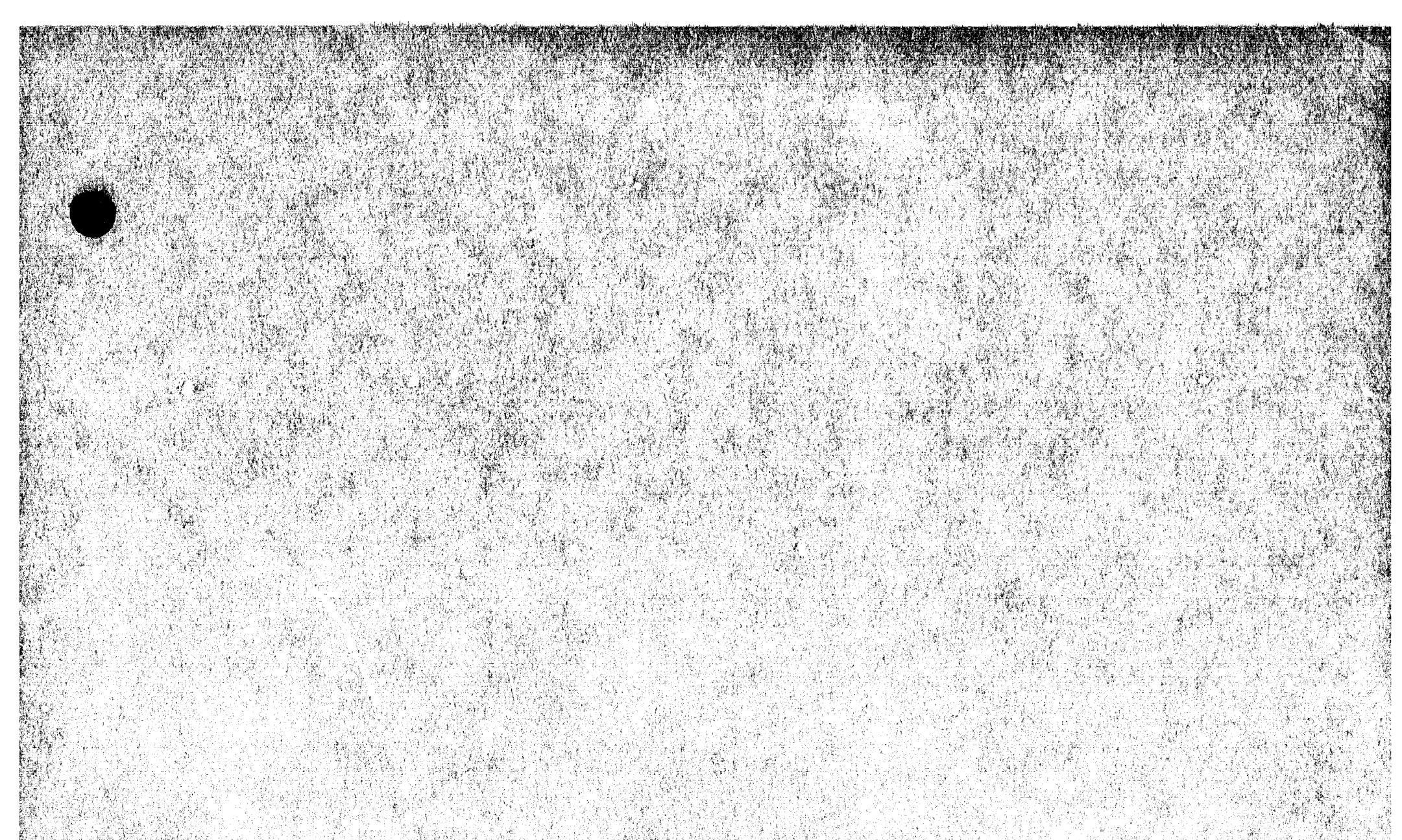

\section{APPENDXX C IMPORT/EXPORT INFORMATION AND REQUIREMENT,}




\section{APPENDIX C \\ MMPORT/EXPORT INFORMATION AND REQUIREMENTS \\ 1. MMPORTING INTO THE UNITED STATES}

\section{$1.1 \quad$ INTRODUCTION}

Communication between the parties involved in a commercial import transaction is necessary to avoid problems that can occur after a shipment arrives at a U.S. Port of Entry, such as:

- Assessment of excess duty through misclassification or buying from nations that do not enjoy most-favored-nation status.

- Assessment of duty on a piece of foreign equipment (on which duty was previously paid) being returned to the United States after having been exported for repair or alteration by a foreign vendor, or assessment of duty on a complete article that is comprised. in part, of American goods previously exported.

- Detention of urgently needed goods at the port for want of correct documentation. [The cure for missing documents is to post a bond until the documents can be produced (see 19 CFR 141.66).]

- Penal sanctions affecting the importer through inaccurate or misleading statements of fact or umissions of required information even though unintentional. Mismarking is subject to additional duty of $10 \%$.

- Duty-free entry of scientific instruments or apparatus provided that: (1) the application (Form ITA-338P) is executed in the name of the U.S. Department of Energy (DOE) and is signed by a cognizant official of the Department, and (2) the procurement document is executed bv DOE.

- Duty-free entry of articles exported for scientific or educational purposes and returned to the United States. (See 19 CFR 10.67 for details.)

As an agency of the Federal Government, DOE enjoys certain privileges and exemptions from the bonding requirements and the regulations of the Customs Service.

- No bond or deposit of estimated duties or taxes is required to release a shipment if a stipulation of compliance in the name of DOE is filed with Customs. Customs will bill DOE after liquidation (see 19 CFR 141.102). However, a few DOE contractors import under their own names. If they employ a customshouse broker, either their bond or the broker's bond can be used as surety. 
- No bond is required to release shipments under an Immediate Delivery Permit provided that a stipulation in the form provided in 19 CFR 10.101 is filed with Customs.

- $\quad$ Certain source materials (Item 9808.00.5000 HTSUS) are accorded duty-free status when so certified by DOE in the form shown in 19 CFR 10.102 .

- $\quad$ American goods returned (Item 9801.00.10 HTSUS) enjoy duty-free status when certified on the letterhead of the Department or contractor in the form shown in 19 CFR 10.103. For better control, some contractors elect to register American goods to be returned on Customs Form 4455 (Figure 18) when exported and execute Form 3311 (Figure 19) when imported. Also see 19 CFR 10.67 for articles returned after having been exported for scientific or educational purposes. Use Forms 4455 and 3311 when applying these provisions.

\subsection{CUSTOMS ORGANIZATION}

The Customs Service staffs Ports of Entry in Puerto Rico, the Virgin Islands, and in every State in the Union except South Dakota and Wyoming. See 19 CFR 101.3 for Customs regions, districts, and ports. In addition, there are Customs Officers attached to U.S. embassies in most countries that are U.S. trading partners. Foreign exporters are encouraged to consult with these officers concerning U.S. Customs regulations.

\subsubsection{Customs Regulations}

Imports of goods into the United States are controlled by the following statutes and regulations:

- Omnibus Trade and Competitiveness Act of 1988.

- $\quad$ "Customs Duties," CFR 19, Chapter 1.

- $\quad$ "Harmonized Tariff Schedules of the United States" (HTSUS).

CFR 19 and HTSUS are published by the Government Printing Office and should be included in the reference library of any importer. Another publication of the Government Printing Office, "Importing into the United States," written in laymen's language, is helpful in interpreting Customs regulations. 


\subsubsection{The Harmonized System}

The Harmonized System (HS) is a new classification system for goods moving in international tradie. HS has been adopted by the United States and all of its major trading partners. There are 137 countries also considering adoption. Nomenclature and basis for classification are uniform in the HS, but each country establishes its own rates of duty subject to agreements under the "General Agreement on Tariffs and Trade" (GATT) treaty. Two new documents, HTSUS and the Harmonized Schedule B (HSCH B), replaced the Tariff Schedules of the U.S. Annotated (TSUSA) and Schedule B, respectively, effective January 1, 1989. A comparison of these documents follows.

\begin{tabular}{|c|c|c|c|}
\hline A. & TSUSA $\quad v$ & HTSUS & HSCH B \\
\hline Measure & U.S. & Metric & Same \\
\hline Language & U.S. & Oxford English & Same \\
\hline Construction & $\begin{array}{l}8 \text { Schedules } \\
\text { and parts thereof }\end{array}$ & $\begin{array}{l}22 \text { Sections } \\
99 \text { Chapters }\end{array}$ & $\begin{array}{l}\text { Same } \\
98 \text { Chapters }\end{array}$ \\
\hline Headings & $\mathrm{N} / \mathrm{A}$ & 5,000 6-digit & Same \\
\hline Tariff & 7,208 5-digit & 8,090 8-digit & N/A \\
\hline Statistical & 13,723 7-digit & 10,457 10-digit & *8,000 10-digit \\
\hline $\begin{array}{l}\text { Basis for } \\
\text { Classification }\end{array}$ & $\begin{array}{l}\text { Chief value } \\
\text { Chief use } \\
\text { Ornamentation }\end{array}$ & $\begin{array}{l}\text { Predominant Weight } \\
\text { Essential character } \\
\text { eliminated }\end{array}$ & $\begin{array}{l}\text { Same } \\
\text { Same } \\
\text { Same }\end{array}$ \\
\hline Special & N/A & N/A & Chapter 98 \\
\hline Provision & $\begin{array}{l}\text { Schedule } 8 \\
\text { Appendix }\end{array}$ & $\begin{array}{l}\text { Chapter } 98 \\
\text { Chapter } 99\end{array}$ & $\begin{array}{l}\text { N/A } \\
\text { N/A }\end{array}$ \\
\hline
\end{tabular}

* Of the 8,000 entries, about 5,600 are identical in both the HTSUS and the HSCH B. The difference is attributable to entries for tariff purposes, which are not applicable to exports. 
B.

\section{TSUSA}

Schedule

Part

Subpart

Provision

Item Number

General Head-notes 1 thru 9

General Head--

note 10

General Head--

notes $10(e)$ and $10(i$ and $j)$

\section{HTSUS \& HSCH B}

Section

Chapter

Subchapter

Heading

Subheading

General Notes

General Rules of Interpretation (GRI)

U.S. GRI

C.

\section{EXAMPLE OF STRUCTURE}

\section{UNIVERSAL LEVEL}

Headnotes

GRI

Notes

Section XVIII

Chapter 90

Heading 9008

Subheading 9008.30

\section{NATIONAL (U.S.) LEVEL}

Tariff Subdiv. 9008.30 .00

Statistical Subdiv. 9008.30 .0000

(Nations may expand upon the 6-digit number but may not conflict with or alter it.

Also may add GRIs and notes applicable to that nation's trade.)

D. In the HTSUS, there is a more logical progression in the grouping of commodities starting with raw materiais and progressing through processing to finished goods. Example: Chapter 41, "Raw Hides and Skins (other than Furskins) and Leather"; Chapter 42, "Articles of Leather," etc.; Chapter 43, "Furskins and Artificial Fur; Manufactures thereof." Under the HS, "Goods" is always interpreted as "finished goods."

E. One of the objectives in converting the TSUSA to the HS was to minimize tariff-rate changes. This objective was met by holding all changes to $1 / 2$ of $1 \%$. However, there were some very drastic changes, mostly reductions, on some commodities. For example, a trade weighted average (TWA) was made of ornamented $v$. nonornamented cotton overcoats imported into the United States. Statistics proved that most of the trade was in nonornamented. Subsequently, "ornamentation" was dropped as a criterion and the tariff rate reduced from $21 \%$ to $10 \%$. 
F. "Essential Character" is the backbone of the new classification criteria under the HS. Its definition and an example follow.

"It is the role of the component or material in relation to the use or function of the goods." For example, an office desk with a wooden top, steel legs, and drawers would be classified under 9403.10 .00 as "metal furniture of a kind used in offices" inasmuch as it is the drawers rather than the top that gives the desk its essential character. Otherwise, the product would be a table with the wooden top giving it its essential character.

G. While there still are some specific entries for sets, i.e., 8206 "Hand Tool Sets," the HTSUS has a broad provision for the importation of nearly any merchandise put up as a set provided it meets the following definition:

"A multitude of items having a single function with a single classification."

1. They must consist of at least two different articles that are classifiable under different headings.

2. At the time of entry or export. they must be put up as a set suitable for retail sale.

3. They must consist of articles put up together to meet a particular need or carry out a specific activity.

Example: A pasta meal for two consisting of 0406 package of grated cheese, 1902 package of uncooked spaghetti, and 2103 can of tomato sauce would be classified as a set under 1902.

This also applies to systems with a single function and sold as a system.

H. This new philosophy of togetherness allows items normally sold with, and shipped with, mixtures and sets to be classified as a single item. Example: $A$ framed painting can be grouped under a single heading provided the frame is of a kind nornally sold therewith, but not a $\$ 2,000$ frame with a cardboard poster.

I. There are six universal GRIs and one U.S. GRI that are the keys to classification.

GRI \#1 - "Eo Nomine" or earliest use by its own name.

GRI \#2 - "Essential character principle" for composite goods and mixtures. Example: A motorcycle without a motor is still a motorcycle since it retains its essential character. 
GRI \#3 - "Essential character principle" for finished goods. Example: The steel desk with the wooden top previously cited.

GRI \#4 - "Most akin rule" if previous GRIs do not fit.

GRI \#5 - "Shipped with principle" for cases and packaging material. Does not apply to reusable containers like compressed-gas cylinders.

GRI \#6 - Compare apples with apples, not oranges, when determining classification. Only subheadings at the same level are comparable.

U.S. GRI - Controlled and actual use. Plan your use and use your plan.

GRIs are hierarchical; that is, they must be considered in the order listed with GRI \#1 having first priority.

J. Notes have the same legal weight as headings or subheadings. They are intended to:

1. Define the scope of limits of a heading or subheading.

2. Provide lists of goods excluded from a particular section.

3. Provide lists of goods included in a particular section.

It is very important that notes be considered when classifying commodities.

K. Explanatory Notes are contained in a separate four-volume, 1,620-page "official" explanation of what was intended by the GRIs, section and chapter, notes and headings. These notes provide the authoritative source for technical definitions, some of which were used in this writeup.

\subsection{THE ENTRY PROCESS}

Basically, entry is a two-step process. First, file the documents to allow the merchandise to be imported. Second, file the documents that will allow Customs to assess duty or exempt the merchandise from duty and obtain statistical information. The steps follow.

1. Within 5 working days of the date of arrival of a shipment at a U.S. Port of Entry, entry documents must be filed unless an extension is granted. These documents are:

(a) Entry Manifest, Customs Form 7533, or Application and Special Permit for Immediate Delivery, Customs Form 3461, or other form of release 
required by the District Director of Customs. See 19 CFR 141.61. An Entry Summary, Form 7501 (Figure 20) also may be filed if the necessary documents and information needed to complete the form are available within the 5 days.

(b) Evidence of the right to make entry as the Importer of Record is required. Presentation of shipping papers and invoices in the name of the importer is usually sufficient evidence. See 19 CFR 141.11. Customshouse brokers also have the right to make an entry.

(c) Commercial invoice or a pro forma invoice (Figure 21) when the commercial invoice cannot be produced. See 19 CFR 141.86 for general contents and $141.87,141.88$, and 141.89 for additional information required for certain classes of merchandise such as aluminum metal, bearings, machines, iron and steel articles, colors, dyes and stains, etc.

Fifteen exceptions are listed in 19 CFR 141.83; four examples are listed below.

- $\quad$ Merchandise having an aggregate price of value of $\$ 500$ or less.

- Merchandise returned to the United States after having been exported for repairs or alteration. Duty is applicable only on the cost of the repairs or alteration.

- Exported merchandise returned to the United States because it was undelivered.

- Merchandise consigned to or entered in the name of DOE.

Although no commercial invoice is required, some evidence of value and description of the merchandise for statistical purposes must be presented to Customs.

(d) Packing lists if appropriate.

(e) Other documents necessary to determine merchandise admissibility such as a cost of production statement, an NRC license, a certificat:- $n$ on Canadian crude petroleum, a stipulation or certificate by DOE or the contractor, etc.

(f) Following acceptance of the entry, the shipment is examined, or examination is waived and released. 
2. When goods are released from Customs custody on entry documents, an Entry Summary Form 7501 (Figure 20) must be filed and duty paid (or for DOE billed later) at the port of entry within 10 working days of the time the goods are entered and released. Many contractors dispense with entry documents by filing the entry summary within 5 days. Additional information is required on the entry summary when importing petroleum products in bulk. See 19 CFR 151.41 for details.

If there is a failure to file an entry for goods in a timely manner, Customs may place them in a general order warehouse at the expense and risk of the importer.

\subsection{MAIL AND INFORMAL ENTRIES}

Contractors may find it advantageous to use the mails to import low-valued merchandise provided:

- The shipment does not exceed the size and weight limitations of exporting country.

- The merchandise does not exceed $\$ 1,000$ in value except for certain classes of textiles, wood products, wearing apparel, plastic or rubber articles, agricultural products, and products of the seas, on which the limit is $\$ 250$. These exceptions are listed in Sections II, VII, IX, and XI of HTSUS.

The duties on parcels not exceeding the dollar limits above are collected by the letter carrier or, if for DOE, the parcel may be released upon arrival if accompanied by the stipulation explained in Section 1.1 of this appendix. The stipulation can be sent to the foreign vendor with the order. No entry is required on duty-free merchandise not exceeding $\$ 1,000$.

The vendor should be instructed to mark the package with the country of origin and enclose two copies of a commercial invoice with the mark "Invoice Enclosed." If the parcel is sealed it should be marked "May be opened for Customs purposes before delivery." Certain types of merchandise such as wood products and textiles require other marks, but DOE contractors do not usually import such commodities. See 19 CFR 145 for details. The same dollar limits apply to certain classes of merchandise eligible for informal entry. See 19 CFR 143.21 for details.

\subsection{POWER OF ATTORNEY}

When entry is made by a customshouse broker, a Customs power of attorney (Form 5291) given by the firm for which the broker is acting as agent should be made (19 CFR 141.46). The best evidence of the authority of an employee to make entry for his employer is a Customs power of attorney. Generally, however, Customs will also accept the signature of a traffic officer of the importing corporation if it is well known. DOE field offices may delegate to a contractor employee the authority to act for DOE by writing Customs at the port of entry on DOE letterhead. 


\subsection{EXAMINATION OF GOODS (19 CFR 151)}

Prior to release of the goods Customs may designate a representative quantity for examination or may waive examination for DOE. Examination is necessary to determine:

- The value of the goods and their dutiable status.

- That the goods were properly marked especially if the regulations require special markings or labeling (19 CFR 11).

- Whether or not the shipment contained prohibited articles (19 CFR 145, Subpart E).

- That the goods are correctly invoiced.

- Whether or not the goods are over/short from invoiced quanities.

- That the tare weight allowance appears to be reasonable on goods assessed duty based on weight (19 CFR 159 Subpart B).

\subsection{THE COMMERCIAL INVOICE}

A commercial invoice, prepared in accordance with 19 CFR 141.86 , and signed by the seller, shipper, or their agents is acceptable. The invoice must contain the following information:

- Port of entry to which the merchandise is destined.

- Names of buyer and seller (or shipper and receiver) and time and place of sale.

- A detailed description of the merchandise, including marks.

- The quantities in weights and measures of the United States or exporting country and number of packages.

- Purchase price in the currency of the sale.

- The kind of currency.

- All charges upon the merchandise itemized by name for freight, insurance, commission, and all other expenses for bringing the merchandise alongside the carrier at the first port of entry. Packaging and inland freight in the foreign country need not be itemized if included in the invoiced price. 
- All rebates, drawbacks, bounties, discounts, separately itemized.

- Additional information for certain classes of merchandise (19 CFR 141.89).

- Invoices and attachments in English or accompanied by a translation.

\subsection{OTHER INVOICES}

When the required commercial invoice is not available at time of entry, a pro forma invoice executed by the importer may be substituted by posting a bond against production of the invoice for statistical purposes within 50 days but not later than 6 months from date of entry.

A special summary steel invoice in duplicate is required on certain iron and steel products with an aggregate purchase price of $\$ 5,000$ or more from contiguous countries or $\$ 10,000$ from other countries. This invoice must accompany the entry summary. See 19 CFR 141.89 for details. In addition to the special steel invoice required by U.S. Customs, some countries like Japan control exports of steel in any quantity to the United States by issuing a special export license.

\subsection{CUSTOMS DUTY}

All goods imported into the United States are subject to duty or duty-free entry according to their classification in HTSUS.

There are three types of duty rates: ad valorem, specific, or compound rates. The ad valorem rate is used most often and is applied to a percentage of the value appraised by Customs. A specific rate is the rate applied to a unit of measure, weight, number of items imported, dozens, etc. A compound rate is a combination of the first two such as 5 cents per pound plus $5 \%$ of the appraised value.

Duty varies by country, as reflected in the three columns headed "Rates of Duty" in HTSUS. Column 1 affords preferential duty status to U.S. trading partners such as the United Kingdom; Column 2 levies high statutory rates on imports from Eastern Bloc nations; and the "Special" column reflects free or minimum-duty rates to promote the development of the economies of friendly or Third World nations.

The rates in the "Special" column reflect the following preferences:

Genex:..ized System of Preferences.

Automotive Products Trade Act

Agreement on Trade in Civil Aircraft.

United States-Canada Free Trade Area.

Caribbean Basin Economic Recovery Act.

U.S.-Israel Free Trade Area

\author{
A or $\mathrm{A}^{*}$ \\ B \\ C \\ CA \\ $\mathrm{E}$ or $\mathrm{E}^{*}$ \\ IL
}


Using HTSUS Item 8205.10.00, "drilling, threading and tapping tools," as an illustration, Column 1 rates would be $6.2 \%$ ad valorem; Column 2 would be $45 \%$ ad valorem: country CA, $5.5 \%$ ad valorem; and countries A,E,IL, duty free. To claim exemption from duty, see 19 CFR 10.172, which requires a certificate of origin. With the possible exception of Israel, countries afforded duty-free status might not produce the type or quality of products that would attract DOE or contractor buyers. See 19 CFR 10.171-10.198 for the Generalized System of Preference and the GRI and notes in HTSUS. Also see 19 CFR 10.179 for the duty-free entry of Canadian crude petroleum under an exchange agreement.

\subsection{DRAWBACK - REFUND OF DUTY}

A 99\% refund of duty is allowed for any imported merchandise found not to conform to specification or shipped without consent of the consignee. Rejected merchandise should be returned to Customs for examination and exported within 90 days of its release unless an extension is granted. See 19 CFR Part 191 for details on this and other types of drawbacks. Apply for a drawback on Customs Form 7539 (Figure 22) supported by Customs Form 7511 and the shippers export declaration.

\subsection{TEMPORARY IMPORTATION UNDER BOND}

Section XXII, Chapter 98 of HTSUS admits certain articles into the United States duty free for a period of 1 year. A bond in the amount of double the estimated duty must be posted. Of interest to DOE and its contractors might be Item 9813.00.51 "Professional equipment, tools of the trade, repair components for such equipment," which are duty free.

\subsection{ATA CARNETS}

ATA stands for the combined French and English words Admission Temporaire-Temporary Admission. The carnet is an international customs document that may be used for the temporary admission of protessional equipment, commercial samples, and advertising material. Carnets can be used in any country that is a U.S. trading partner and certain others that have accepted the ATA convention. In the United States, carnets are issued by the U.S. Council of the International Chamber of Commerce, 1212 Avenue of the Americas, New York, NY 10036. There is a fee for issuing the carnet and guarantee against the payment of Customs duties. The term is 1 year, not renewable.

Another type of carnet is the Transport International Routier (TIR), which is used between countries outside of the Common Market. The TIR convention permits sealed cargoes to move from one country to another. Each cargo is covered by a Customs bond guaranteeing delivery of the goods to the destination country. 


\subsection{CONVERSION OF CURRENCY}

The conversion of currency for Customs purposes is at the rate of exchange determined by the Federal Reserve Bank of New York. The rate used during the quarters is the one certified on the first business day of each calendar quarter unless fluctuations of $5 \%$ or more occur. The certified rate for the day on which the fluctuation occurs will then be used. The date of exportation of the goods is used to determine the certified rate of exchange for Customs purposes, even though a different rate is used for payment of the goods.

\subsection{RESTRICTED OR PROHIBITED ARTICLES AND OTHER AGENCY REQUIREMENTS}

Restrictions or prohibitions on food; drugs; cosmetics; economic poisons; hazardous substances; and dangerous, caustic, or corrosive substances may be found in 19 CFR 12 . Enforcement of laws for other agencies including NRC is shown in 19 CFR 161.2. DOE is exempt from NRC import regulations. See Section 1.18 of this appendix.

The Toxic Substances Control Act (TCSA) holds importers responsible as if they were "manufacturers" of toxic, noxious, or hazardous materials at ports of entry. Under this law the importer must produce health and safety data, provide premarketing notification, and review the regulation of new substances for new uses. Included are chemicals fully contained in dyes, paints, instruments, and machinery.

\subsection{FOREIGN TRADE DEFINITIONS}

The foreign seller and the U.S. buyer should agree in the sales contract as to which terms of sale will govern the contract, either the "Revised American Foreign Trade Definitions 1941" (similar to FAR 47) or the Incoterms of 1980. There are some subtle differences in the assumption of risk, which could lead to misunderstandings. Also, when buying by weight, "ton" should be defined as metric (2,204.6 pounds).

\subsection{FINANCING IMPORTS}

Most DOE contractors have credit ratings that enable them to purchase foreign goods on open account often through a distributor in the United States. Other methods involve using banks as collection agencies.

The most secure method of collection is through an irrevocable letter of credit, which the importer opens with a U.S. bank. The foreign bank, acting as correspondent for the U.S. bank, will remit to the seller when the terms of the letter of credit are satisfied. Usually the seller draws a draft, attaches the necessary clearance documents, and sends the draft through the foreign bank for payment. Once payment is made there is no recourse against the seller. 
If a business relationship exists between the parties, the seller may depend on a sight draft or time draft $(30,60$, or 90 days) to effect collection. The seller attaches the necessary clearance documents and forwards the draft with a collection letter through the correspondent banks with instructions to transfer title when the buyer has satisfied or guaranteed payment.

\subsection{DUTY-FREE ENTRY OF SCIENTIFIC INSTRUMENTS OR APPARATUS}

Item 9810.00 .60 of HTSUS provides for duty-free importation of instruments and apparatus "entered for the use of any nonprofit institution whether or not public or private established for educational or scientific purposes...if no instrument or apparatus of equivalent scientific value....is being manufactured in the United States" (15 CFR 301.1).

Applications must be made on Form ITA-338P, which may be obtained from the Statutory Import Staff, International Trade Administration, U.S. Department of Commerce, Washington, DC 20230 (15 CFR 301.3). Applications may be filed by eligible persons who have placed a bona fide order for the article or who intend to place a bona fide order within 60 days after decision on the application becomes final. For DOE, an eligible person can be a cognizant official of the Department. However, a contracting officer of the field office having jurisdiction is preferred.

The application is filed with the U.S. Customs Service at the address shown on page 1 of the application. Customs reviews the nonprofit status of the applicant in the current edition of "Cumulative List of Exempt Organizations," or verifies that the applicant is an agency of the U.S. Government involved in educational or scientific work. Customs assigns numbers to the application and also verifies that the instrument or apparatus falls within the class eligible for duty-free entry in the U.S. Notes to Subchapter X, HTSUS. It is important to justify domestic nonavailability not just with such a statement, but also with a detailed description of the instrument or apparatus on the application. Such detail is necessary to enable Customs to make its determination as to eligibility for duty-free entry and to avoid a protest from domestic suppliers when the application is posted for public inspection.

Customs assigns a number to the application and forwards one copy to the Secretary of Health and Human Services, the original and one copy to the Department of Commerce, keeps a file copy, and returns one copy to the applicant stamped "Accepted for Transmittal to the Department of Commerce."

The Department of Commerce (DOC) posts a copy for public inspection at its Washington, DC, offices within 10 days of receipt and publishes a notice in the Federal Register. If no adverse comments are received within 20 days, the application will be approved.

In the meantime, the copy ret"rned to the applicant stamped "Accepted for Transmittal" may be used with the other entry documents to avoid a deposit of estimated duty. Liquidation will be suspended for 180 days pending approval of the application. 
When the imported instrument reaches the port of entry, a claim must be filed by the importer for duty-free entry under HTSUS Item 9810.00 .60 at the same time a bond, a deposit, or, in the case of DOE, a stipulation must be posted against payment of duty should the application be denied. If no such claim is made, the instrument will be classified without regard to Item 9810.00 .60 and duty will be assessed and entry liquidated in the usual manner. See 15 CFR 301 for these details.

Note: The names of commercial firms who are DOE contractors may not be shown as either the purchaser or the consignee except that the instrument may be shipped to DOE in care of such contractor.

\subsection{NUCLEAR EQUIPMENT AND MATERIAL IMPORT PROCEDURES}

Imports of nuclear materials into the United States are controlled by the following statutes and regulations in addition to those of the Customs Service:

- Atomic Energy Act of 1954 as amended.

- $\quad$ Nuclear Non-Proliferation Act of 1978.

- $\quad 10$ CFR 110 "Export and Import of Nuclear Equipment and Material."

- $\quad 49$ CFR 171.11 "Use of ICAO Technical Instructions," 171.12 "Import and Export of Shipments," and Part 171.12a "Canadian Shipments and Packaging."

10 CFR 110.27 addresses imports as follows:

A General License is issued to any person to import byproduct, source, or special nuclear material, other than 100 kilograms or more of irradiated fuel, if the consignec is authorized to possess the material under: (a) a contract with the Department of Energy or (b) an exemption from licensing requirements issued by the Commission, or a general or specific license issued by the Commission or a State with which the Commission has entered into an agreement under Section 274b of the Atomic Energy Act. Importers of special nuclear material under this General License shall provide advance notification of imports to the Commission as specified in 10 CFR 73.27.

"Person" in 10 CFR 110.2 is defined as: "any individual, corporation, partnership, firm, asscciation, trust, estate, institution, group, Government agency other than the Commission (NRC) or, with respect to imports, the Department of Energy...."

The Department is exempt from the provisions of Part 110 of 10 CFR and exercises its own import controls through the Office of Nuclear Non-Proliferation Policy (IE-13). This office approves the import with the concurrence of the Office of Security Affairs (DP-323.1) after a noninimical determination, if required. 
DOE imports of special nuclear materials (SNM), source, or byproduct materials as a government-to-government shipment require the authorization of IE-13 on Form MB\#4 shown as Figure 23. This form must be presented to Customs at the port of entry with the other entry documents, or Customs will frustrate the shipment until some authority for entry is produced.

Form MB\#4 may be executed by a field office, a DOE contractor as directed, or by the foreign exporter and submitted to IE-13 for approval after coordinating the details with each other. If DOE-owned material, the control number will be the foreign contract number that was assigned when the material was exported. After the approved Form MB\#4 has been distributed to the parties by IE-13, contract negotiations can be finalized. If foreign-owned material, the contract should state disposition of the material upon completion of use.

Preparation and filing of entry documents and transportation arrangements from the port of entry are the responsibility of the receiving contractor. The contractor may deal directly with Customs or through a customshouse broker. Inland transportation is subject to those parts of 49 CFR referenced in Section 1.18 of this appendix.

Upon receipt, the receiving contractor shall execute a 741 Form (See DOE Order 5633.5, Nuclear Materials Reporting and Data Submission Procedures). Oak Ridge will enter the transaction into the International Nuclear Material Tracking System upon receipt of its copy.

\section{EXPORTING FROM THE UNITED STATES}

\subsection{INTRODUCTION}

The movement of goods internationally is quite complicated. Skills in international documentation and logistics are required to administer a successful program. Inexperienced personnel would be well advised to rely on the advice of a foreign freight forwarder until some expertise can be acquired through training and experience. Various organizations publish and sell guides that are helpful, such as:

- A Guide to Export Procedures.

- A Guide to Export Documentation (with sample documents).

- A Guide to International Shipping.

- A Reference Guide to the Export Administration Regulations. (Explains licensing and the commodity control list.)

- The Official Export Guide published by North American Publishing Company, 401 North Broad Street, Philadelphia, PA 19108. 
DOE produces only a few commodities, which it markets commercially in international markets. Many of its exports are of nuclear equipment, materials, and related scientific instruments. These commodities are subject to the licensing requirements of both the NRC and the Department of Commerce for national security, nuclear nonproliferation, and foreign policy reasons. Defense related articles and technology are controlled by the Department of State (see Section 3.1.1 of this appendix). As a result, this section will cover commercial transactions only briefly. Traffic personnel will not be involved in all aspects of the export but should understand the overall procedures.

\subsection{THE COMMERCIAL TRANSACTION}

In a commercial transaction the seller offers a product at a given price to the buyer at some geographic location. In turn, the buyer agrees to purchase the product at the stated price and to take title at the point agreed upon. Each has certain responsibilities to carry out according to the terms of sale. Also see Section 1.15 of this appendix regarding foreign trade definitions. Depending upon the skills of the principals some responsibilities may be discharged by the principals and some may be contracted to a carrier or a foreign freight forwarder.

\subsubsection{General Licenses}

A "General License" is a broad grant of authority by the Federal government to all exporters for certain categories of commodities to all or most destinations. Most U.S. exports are shipped abroad under various general licenses, and no application is required tor the use of these licenses except for general license GTE. If, after reviewing the Export Administration Regulations, the exporter determines that a validated license is not required, the exporter may ship under a general license. Several types of general licenses are shown below. A description of these and others may be found in 15 CFR 771.

GCT General license for "a" level commodities to COCOM participating countries except those specifically excluded

G-DEST General Destination (first choice)

G-DR General license - Germany

G-FW General license - free world

GLR General license - return

GLV General license - limited value

GTE General license - temporary imports - requires specific approval for temporary import not exceeding 12 months 
G-COCOM General license to COCOM participating countries

GCG General license to cooperating governments

GTDA General license - technical data to all destinations

GTDR General license - technical data under restriction.

\subsection{ADMINISTRATIVE CONTROLS}

The following regulations govern the exporting of nondefense, nonnuclear materials and equipment from the United States:

Title 15, Parts 700-799 "Export Administration Regulations," Bureau of Export Administration, Department of Commerce. Also Parts 768-799 are available as a looseleaf edition.

HSCH B, "Statistical Classification of Domestic and Foreign Commodities Exported from the United States."

\subsection{QUOTATIONS AND PRO FORMA INVOICES}

Only the seller can make a price quotation, which can be in many forms: telephone, cable, letter, or a pro forma invoice. In order to accumulate all of the charges that will be applicable to shipments to the buyer's country, the seller must develop the import requirements of that country. A consulate of the buyer's country is one source. Another is the Export Shipping Manual published by the Bureau of National Affairs, Inc. (BNA).

If the seller is making frequent quotations involving ocean freight, a quotation worksheet is recommended. See page 3-34.

If a Cost, Insurance, Freight (CIF) pro forma invoice is requested, it may be prepared on the firm's billhead. As each article is priced and extended, enter directly below the dimension of each package in centimeters or meters, then the cube of each package in meters and the weight in kilograms plus totals. Total the merchandise to a Free On Board (F.O.B.) origin price, then add international transportation, forwarders fees, and insurance to arrive at CIF destination price. If the article is to move via ocean freight, a breakdown of the elements that comprise the international freight total is necessary. Forwarding fees may be lumped as a total. Some countries require a certification in the language of that country. The pro forma invoice must be prepared with great care because the letter of credit, the purchase order, and the credit documents all will reflect the details contained in the pro forma invoice. When the commercial invoice is issued it should be an updated version of the pro forma invoice. It should reflect the actual shipping information plus package markings and other information supplied by the buyer such as the purchase order number, import license number, and dates. 


\subsection{INSTRUMENTS OF PAYMIENT}

See Section 1.16 for credit documents used in foreign trade. For exports the procedure would be the reverse of that shown for imports. Anyone dealing in drafts or letters of credit regularly should secure the following publications from the International Chamber of Commerce, 1212 Avenue of the Americas, New York, NY 10036:

- $\quad$ Publication No. 322, "Uniform Rules for Collections."

- Publication No. 400, "Uniform Customs and Practice for Documentary Credits."

Credit documents are not usually the province of traffic personnel except to the extent that they contain transportation information.

\subsection{FOREIGN DMPORT LICENSES}

Foreign countries control and license the importation of goods for several reasons: shortage of exchange dollars, protection of local industry, national security, and statistics for duty purposes. Some countries require the buyer to furnish a copy to the seller to be submitted to the consulate at the time the commercial or consulate invoices are visaed by the consulate (for a fee). Others require only that the license number and date be entered on the commercial invoice. Still others have no requirement other than issuance of the license to the buyer.

\subsubsection{Import and End-Use Certificates}

Refer to 15 CFR 775 for destinations requiring International Import Certificates and EndUse Certificates to be furnished by the importing country and Delivery Verification Certificates to be secured by the exporter.

\subsubsection{Letters of Assurance}

When exporting technical data, review 15 CFR 779 concerning the restrictions on licensing of technical data (including software) and specifically the requirement for a letter of assurance from the foreign importer that he/she will not knowingly re-export or otherwise disclose, directly or indirectly to certain country groups, any software or other technical data received from the exporter.

\subsection{THE COMMERCIAL INVOICES}

The commercial invoice is the final statement of the agreement between the seller and buyer. In the course of the transaction it will be examined by everyone party to the transportation and payment of the goods. 
- The carrier or inturnational freight forwarder.

- The consulate of the buyer's country if required.

- The seller's bank if not sold on open account.

- The Customs officer in the buyer's country.

- The buyer's bank.

- The import broker.

- The insurance company in event of loss.

If the seller does not make the agreement explicit on the commercial invoice, each of the above is free to make its own interpretation, not always in favor of the seller.

A common error that occurs on computer-created invoices is to designate the "ship to" address as that of the international carrier or freight forwarder, neither of whom performs as the buyer's agent. The "ship to" address is the same as the consignee on the international bill of lading, which is usually the foreign bank or to order of the shipper.

\subsection{FOREIGN CUSTOMS INVOICES}

Some countries including Canada (Figure 24) require a customs invoice for use by foreign Customs officers. Standard Customs invoices are available at many stationers specializing in transportation forms and are not difficult to fill out. Customs invoices must be included with other entry documents.

\subsection{CONSULAR INVOICES}

The consular invoice can usually be purchased from the resident consulate. It usually must be submitted to the consulate for legalization or visa upon shipment. Latin American countries are the most frequent users of consular invoices. Consular invoices must be included with the other entry documents.

\subsection{SHIPPER'S LETTER OF INSTRUCTIONS}

There are many forms of the shipper's letter of instruction. The freight forwarder may supply forms or the forms may be purchased from stationers specializing in transportation forms. There are several types: air direct, ocean containerization, and ocean pier to pier. The letter of instruction should state:

- The name of the ultimate consignee. 
- The name of the intermediate consignee, such as the foreign bank.

- The number of pieces, the type packaging, and the description of the goods in $\mathrm{HSCH}$ B vernacular, the gross weight and dollar value by $\mathrm{HSCH} B$ number.

- The port marks to appear on packages or containers.

- U.S. license specifications, general (15 CFR 771), or Specific (15 CFR 772-775).

- List of documents that accompany the letter.

- Freight prepaid or collect.

- Special instructions concerning completion and distribution of documents.

\subsection{SHIPPER'S EXPORT DECLARATION}

The Shipper's Export Declaration (SED), Department of Commerce Form $7525-\mathrm{V}$, is required for:

- Shipments from the United States exceeding $\$ 2,500$ in value [15 CFR 786.1(c)( 2$)$ ]; mail ex ding $\$ 500$ [15 CFR 786.3(f)(2)]. The $\$ 2,500$ exemption does not apply to ts requiring (1) a DOC export license, (2) a DOS, Office of Defenst Controls export license under the International Traffic in Arms Regusutions (ITAR), 22 CFR 121-130, (3) inose subject to ITAR but exempt from licensing, or (4) a Department of Justice, Drug Enforcement Administration export permit, 21 CFR 1312.

- All shipments of controlled commodities regardless of value (15 CFR 799).

- Cargo destined to restricted countries (15 CFR 785).

The SED functions both as a census for the statistics on exported commodities and as a regulatory document to control commodities for foreign policy, national security, nuclear nonproliferation, and short-supply reasons. See 15 CFR 786.3 for details. The SED is reproduced as Figure 25 together with instructions for completion. Once the SED is completed it is submitted with the air waybill or the ocean bill of lading to the carrier. The carrier attaches three copies of the SED to the manifest of the aircraft or vessel and submits them to U.S. Customs. 


\subsection{BOOKING THE SHIPMENT}

As soon as an actual shipping date can be established the shipment should be booked with the international carrier as far in advance as possible, especially if the movement is to be on a vessel. The letter of credit could expire while waiting for another ship if the planned ship date is missed. Shipments can be booked through the freight forwarder or direct with the carrier.

\subsection{INTERNATIONAL SHIPPING DOCUMENTS}

International air waybills diffe- frcm ocean bills of lading in that the air waybill is nonnegotiable. If consigned to the buyer, the buyer can take possession of the goods without paying the draft. An alternative is to consign the shipment to the buyer's bank and send the draft with the original air waybill and other entry documents to the bank for collection. Ocean bills of lading may be consigned either to the order of the shipper or foreign bank and title to the goods transferred by endorsement after the draft has been satisfied.

\section{U.S. EXPORT CONTROL AGENCIES}

\subsection{TYPES OF EXPORTS CONTROLLED BY THE AGENCIES**}

$\begin{array}{llll}\text { Agency } & \text { Commodity or Service } & \text { Control Mechanism } & \text { Advisory Agencies } \\ \text { DOC } & \text { General merchandise } & \text { General License } & \text { Treasury } \\ \text { DOC } & \begin{array}{l}\text { All dual-use commod- } \\ \text { ities and technology** }\end{array} & \text { Validated License } & \begin{array}{l}\text { DOS, DOD, DOE, } \\ \text { Treasury }\end{array} \\ \text { DOS } & \begin{array}{l}\text { Defense articles and } \\ \text { services (OMC); } \\ \text { Dual-use commodities } \\ \text { (COCOM) }\end{array} & \text { Validated License } & \text { DOC, DOD, DOE } \\ \text { NRC } & \begin{array}{l}\text { Nuclear equipment and } \\ \text { materials }\end{array} & \text { Validated License } & \text { DOS, DOD, DOE, } \\ \text { DOE } & \begin{array}{l}\text { Nuclear materials, } \\ \text { equipment, technology; } \\ \text { subsequent arrangements } \\ \text { government-to-government; } \\ \text { foreign nuclear activities }\end{array} & \begin{array}{c}\text { Validated License } \\ \text { Det Noninimicality }\end{array} & \begin{array}{l}\text { DOS, DOD, NRC, } \\ \text { DOC, ACDA }\end{array} \\ & & \end{array}$


DOC-Department of Commerce

DOS-Department of State

DOE-Department of Energy

DOD-Department of Defense

NRC-Nuclear Regulatory Commission

ACDA-Arms Control and Disarmament Agency

** Except for a small number of items licensed by other agencies that are not relevant here (e.g., alcohol, tobacco, and firearms under Treasury).

\subsubsection{Regulatory Relationship Between the Agencies}

The Arms Export Control Act (AECA) authorizes controls primarily on exports that are deemed to be inherently military in character or were specially designed, modified or engineered for military applications. The AECA authorizes the control of expor's of "defense articles and defense services to further world peace and the security and foreign policy of the U.S...." The State Department controls these defense articles and services under the International Traffic in Arms Regulations (ITAR), 22 CFR 120 et seq.

The Office of Munitions Control (OMC) in the State Department determines which articles and services are deemed to be defense-related and lists them on the U.S. Munitions List, 22 CFR 121.1. Items on this list are regulated solely under ITAR.

Generally, NRC and DOE regulations take precedence over ITAR in the export of nuclear materials, services, and equipment; but the ITAR will take precedence over the Export Administration Regulations (EAR) in the export of defense articles, related technical data, and defense services.

Most exports of commodities and technical data not controlled under ITAR are controlled under the EAR, 15 CFR 769 et seq. as authorized by the Export Administration Act of 1979. These regulations are administered by the Office of Export Licensing (OEL), Bureau of Export Administration (BXA), and the U.S. Department of Commerce. A license under these regulations is required prior to exporting an EAR controlled commodity or technical data out of the U.S.

The regulatory relationship between the Department of Commerce's Bureau of Export Administration (BXA) and the Department of the Treasury's Office of Foreign Assets Control (OFAC) can be confusing for exporters to understand. OFAC is the office primarily responsible for implementing broad economic sanctions against specific countries. OFAC controls participation by U.S. persons, including foreign subsidiaries in transactions, including imports and exports, with specific countries or nationals of such countries. By contrast, BXA controls exports and re-exports of U.S. origin items whether made by a U.S. or foreign person. Since both agencies possess export licensing authority, some jurisdictional overlap 
exists. While an export license authorized by BXA is recognized by OFAC and OFAC sometimes defers to BXA, exporters or importers contemplating transactions with embargoed or restricted countries should contact OFAC for advice at (202) 376-0392 in case an OFAC license will also be needed. Review also 31 CFR 500-565 "Foreign Assets Control Regulations." OFAC's statutory authorities are (1) Trading With the Enemy Act of 1917, (2) the International Emergency Economic Powers Act of 1977, (3) the Comprehensive AntiApartheid Act of 1986, and (4) the International Security and Development Act of 1985.

DOE regulations in 10 CFR 810 "Assistance to Foreign Atomic Energy Activities" implement Section 57b of the Atomic Energy Act, which empowers the Secretary of Energy to authorize U.S. persons to engage directly or indirectly in the export of "sensitive nuclear technology" for the production of SNM outside the U.S. "Sensitive nuclear technology" embraces a full range of technologies pertinent to nuclear proliferation and national defense. These technologies can include models, prototypes, operational manuals, and technical services. See the congressionally mandated Militarily Critical Technologies List and the DOE Nuclear Technology Reference Book. both of which are classified.

\section{$3.2 \quad$ NUCLEAR-RELATED EXPORTS}

All requests for the export of source materials and special nuclear materials must include a statement, signed on behalf of the recipient nation or group of nations involved, that the proposed export will be subject to the terms and conditions of the appropriate Agreement for Cooperation, and must give the end-use of the nuclear material(s). See Figure 30 for an example of such a statement.

All incoming requests for export to South Africa of stable isotopes, radioisotopes, or materials listed in Section 4.1 of this appendix by DOE or its integrated contractors shall be referred to the DOE Office of Nuclear Non-Proliferation Policy (IE-13).

All requests for export of the nuclear materials specified in Section 4.1 must be referred to IE-13 regardless of the proposed destination.

\section{EXPORT OF NUCLEAR MATERIALS BY DOE}

\subsection{NUCLEAR MATERIAL DEFINED}

The DOE Office of Safeguards and Security designates the nuclear materials to be reported to the Nuclear Materials Management and Safeguards System (NMMSS), administered by the Oak Ridge Operations Office. Export of the following nuclear materials must be reported to the NMMSS International Nuciear Materials Tracking System (INMTS): 
Special Nuclear Material

Plutonium

Plutonium-238

Plutonium-242

Enriched Uranium

Uranium-233

Source Material

Normal Uranium

Depleted Uranium

Thorium
Byproduct Material

Americium-241

Americium-243

Berkelium

Californium-252

Curium

Neptunium-237

Tritium

Other Material

Deuterium

Enriched Lithium-6

Exports of other byproduct materials (e.g., polonium-210) are not reported to NMMSS/INMTS.

In addition to the nuclear materials identified above, nuclear-grade graphite and nuclear equipment are also under export control.

\subsection{ADMINISTRATIVE CONTROLS - NUCLEAR MATERIALS}

Export of nuclear materials from the United States is controlled by the following statutes and regulations:

- Atomic Energy Act of 1954 as amended. See 15 CFR 770.10(e).

- Nuclear Non-Proliferation Act of 1978 as administered by the Departments of State, Energy, and Commerce. This Act is implemented in 15 CFR 778, "Special Nuclear Controls."

- $\quad$ Export Administration Act of 1979 as amended.

- $\quad 10$ CFR 110, "Export and Import of Nuclear Equipment and Material," NRC.

- $\quad 10$ CFR 810, "Assistance to Foreign Atomic Energy Activities."

- $\quad 15$ CFR 700-799, "Export Administration Regulations." Bureau of Export Administration, Department of Commerce. Parts $768-799$ are also available in a looseleaf edition. 
- $\quad$ HSCH B, "Statistical Classification of Domestic and Foreign Commodities Exported from the United States."

- Amendment to Procedures Established Pursuant to the Nuclear NonProliferation Act of 1978, Federal Register, Vol. 49, No. 96, May 10, 1984, pages 20780-20786, amended by Vol. 58, No. 33, February 19, 1991, page 6791 .

- DOE Order 5633.5, Nuclear Materials Reporting and Data Submission Procedures.

\subsection{PACKAGING AND TRANSPORT REGULATIONS}

Effective January 1, 1991, all international shipments of hazardous materials by air or water must comply with the UN packaging recommendations for non-bulk commodities:

- $\quad$ IATA Restricted Articles Regulation published by the International Air Transport Association of Quebec, Canada.

- Regulations for the Safe Transportation of Radioactive Materials, Safety Series 6, issued by the International Atomic Energy Agency (IAEA) of Vienna, Austria. See 49 CFR 171.12 for authority to use IAEA regulations to the port.

- Technical instructions for the Safe Transport of Dangerous Goods by Air, approved and published by the International Civil Aviation Organization (ICAO) of Montreal, Quebec, Canada. See 49 CFR 171.11 for authority to use ICAO Regulations, with certain exceptions for inland transportation to the port.

- International Maritime Dangerous Goods Code, 1988, as amended, published by the International Maritime Organization of London, England, for exports by sea.

- Transportation of Dangerous Goods issued by the government of Canada. See 49 CFR 171.12a.

See álso DOE 5480.3A Chapter VI for approvals, certificates of Competent Authority, DOT validation of foreign packages, special form certifications, and notifications necessary to ship. 


\subsection{GENERAL DOENRC EXPORT CONTROLS}

Export of DOE nuclear material as a government-to-government shipment under a U.S. Agreement for Cooperation with a foreign country requires two or more of the following controls according to the type and quantity to be exported:

- DOE Office of Nuclear Non-Proliferation Policy, IE-13 approves shipment.

- DOE Office of Security Affairs, DP-323.1 concurs in approval upon a noninimicality determination.

- $\quad$ NRC specific export license.

- $\quad$ NRC General License (applicability determined by DOE IE-13).

- $\quad$ Tracking by the INMTS, administered by Martin Marietta Energy Systems, Inc., Oak Ridge, TN. (See Section 4.1 of this appendix.)

Field organizations must seek and obtain DOE IE-13 approval prior to contract negotiations with foreign entities that involve, by any means whatsoever, the distribution of one (1) kilogram quantity or greater of the following materials: uranium enriched in U-235 to $20 \%$ or greater, plutonium, U-233, or heavy water.

All requests for Government distributions (also known as government-to-government shipments) for source, special nuclear, byproduct material, or heavy water, except those involving byproduct material, which are under a General License for export by NRC under 10 CFR 110.23, must be submitted to DOE IE-13 for approval.

DOE export of tritium in quantities greater than $37 \mathrm{TBq}(1,000$ curies) or lithium- 6 in quantities greater than 500 grams to any country must be referred to DOE IE-13 for approval prior to execution of a contract. DP-323.1 must also make a finding of noninimicality.

\subsubsection{NRC General Licenses (DOE IE-13 Authorized)}

A General License (blanket permission) is issued to DOE and its contractors by NRC to export certain quantities of material that fall within the provisions of $10 \mathrm{CFR}$ :

110.21 "Export of Special Nuclear Materials."

110.22 "Export of Source Material."

110.23 "Export of Byproduct Material."

110.24 "Export of Deuterium."

110.25 "Export of iNuclear Grade Graphite."

110.26 "Export. of Nuclear Reactor Components." 
Embargoed destinations are Cuba, Kampuchea, North Korea, and Vietnam (110.28). Restricted destinations are listed in 10 CFR 110.29.

Field offices and contractors inay not export nuclear materials in any quantity under a General License without a determination from DOE IE-13 that a General License does apply. In some instances concurrence by DOE DP-323.1 is required. Byproduct material with atomic numbers 3 to 83 are exempted and may be shipped under General License without DOE IE-13 approval.

\subsubsection{NRC Export Licensed Shipment}

Both an NRC license and DOE IE-13 authorization are required to export quantities of SNM and source materials that exceed the General License limits listed in 10 CFR 110.21 and 110.22. Byproduct material does not require an export license when exported by DOE. Field organizations may delegate the responsibility to the contractor for filing an application directly with NRC. Application must be made on Form NRC-7 (Figure 26) according to instructions shown in Appendix IX, 10 CFR 110.

NRC will return the approved license to the applicant with eight conditions to be followed (Figure 27). When the contractor is the applicant, distribution of copies is made by local procedure and may include:

- Operations Office Nuclear Materials Management Coordinator, Traffic Manager, and program office.

- Contractor's program office.

- DOE-HQ IE-13.

- INMTS.

- DOE-HQ Program Sponsor or NRC Program Sponsor.

Upon shipment, the contractor completes the SED and Form 741, referencing both the foreign contract number and the NRC license number as follows:

$\begin{array}{llll}\text { Foreign contract number } & \text { SED } & & \text { FORM 741 } \\ \text { NRC License } & \text { Block 14 } & & \text { Block 15 } \\ \text { Block 21 } & & \text { Block 16 }\end{array}$

In addition to normal distribution, a copy of the 741 Form should be sent to Martin Marietta Energy Systems, Inc., in Oak Ridge for entry into INMTS. 
The contractor should complete the appropriate shipping documents, attach the SED and other appropriate supporting papers, and release the shipment. If one shipment completes the NRC license, the original copy may be included with the shipping papers. Customs will return the license to NRC; otherwise, a copy may be included and the original returned to NRC after the last shipment has been made.

\subsubsection{Exports Not Requiring a License}

For a government-to-government export approved by DOE IE-13 that does not require a validated license from NRC according to 10 CFR 110, the SED becomes the control document for INMTS. A special SED number must be assigned. The SED should be prepared in the same manner as for a licensed shipment except that "Exempt 10 CFR 110.1" or other appropriate entry should be entered in Block 21 as the authority to export. In addition, an SED continuation sheet should be prepared as shown on the next page.

\subsection{INTERNATIONAL NUCLEAR MATERIAL TRACKING SYSTEM}

Tracking of nuclear material exports is done using INMTS, which is operated by Martin Marietta Energy Systems, Inc., at Oak Ridge. Information is furnished by field installations through copies of material supply contracts, export licenses, Shipper's Export Declarations, and Form 741. Foreign entities report movement from one Agreement for Cooperation entity to another on Forms SER 1 or DOE F-5660.2 and SER 2. See 10 CFR 110.6 "Retransfers."

The initial data entry is from the application for a foreign contract number from field offices, later updated from a copy of the executed contract.

For shipment control purposes, Oak Ridge uses the specific export license number, a General License notation, a special Shipper's Export Declaration number (government-togovernment) or the term "LIC-EXEMPT." Each field office maintains its own series of Shipper's Export Declaration numbers using the first two letters of the RIS symbol for identification. Albuquerque, for example, should be SED-AA-I. Assignment of numbers may be delegated to the contractor.

For additional information, see "Data Collection Procedures, International Nuclear Material Tracking System," Oak Ridge Operations, which is reproduced as Appendix VII.

\subsection{DEPARTMENT OF COMMERCE LICENSES}

The Bureau of Export Adninistration within the DOC maintains a Commodity Control List (CCL), which includes all commodities subject to DOC controls but does not include those commodities exclusively controlled by other Government agencies such as NRC and DOE. 


\section{Certification Statement.}

I certify that the shipment described in this Shipper's Export Declaration as a government-to-government shipment, which does not require a validated license from the Nuclear Regulatory Commission.

"Name:
Title:
Organization:
(The best signator of this document is that of an officer in the Operations
Office having jurisdiction.)

*This information should be typed or printed on the SED.

(See INMTS Data Collection Procedures for the following:)

2. Special SED number.

3. Foreign contract number assigned by INMTS.

4. Nuclear material (NM) description:

$$
\begin{aligned}
& \text { Physical form } \\
& \text { Chemical form } \\
& \text { Material type (in code) } \\
& \text { NM element weight } \\
& \text { Isotope weight percent } \\
& \text { Isotope weight } \\
& \text { NM compound weight (if } \\
& \text { available) } \\
& \text { Hardware and/or } \\
& \text { instrumentation }
\end{aligned}
$$

5. End-use statement.

6. Final disposition of the material upon completion of use at the foreign site.

7. Shipped for DOE by ___ (enter contractor's name) 
There are 10 general categories as follows:

\begin{tabular}{|c|c|}
\hline $\begin{array}{l}\text { Commodity } \\
\text { Group Number }\end{array}$ & Commodity Groups \\
\hline 0 & Metal-working machinery \\
\hline 1 & Chemical and petroleum equipment \\
\hline 2 & Electrical and power-generating equipment \\
\hline 3 & General industrial equipment \\
\hline 4 & Transportation equipment \\
\hline 5 & Electronics and precision equipment \\
\hline 6 & Metals, minerals, and their manufactures \\
\hline 7 & $\begin{array}{l}\text { Chemical, metalloids, petroleum products, and related } \\
\text { materials }\end{array}$ \\
\hline 8 & Rubber and rubber products \\
\hline 9 & Miscellaneous \\
\hline
\end{tabular}

Each individual entry is preceded by a four-digit Export Control Commodity Number (ECCN) and a code letter. The first digit relates to the strategic level of control, the second is the Commodity Group Number, and the next two digits identify related commodities in numerical order within a group. The letter suffix identifies the country groups for which a validated license is required.

Countries are grouped by letter designation in Supplement 1 to 15, CFR 770. For example "S" is Libya, " $Z$ " is North Korea, Vietnam, Cambodia, and Cuba, all of which are embargoed. Canada has no letter designation and is referred to by "name" in the regulations.

Each ECCN entry contains the following information:

- Unit report in dollar value.

- Validated license required by country groups.

- GLV dollar value limit on low-value shipments by countries.

- Processing code (two letters) required on license application.

- Reason for control.

- Special licenses available.

- $\quad$ Special controls (when applicable). 
Some examples of controlled commodities by groups are:

\begin{tabular}{|c|c|}
\hline Group 2-ECCN & $3261 \mathrm{~A}$ \\
\hline Group 3-ECCN & $\begin{array}{l}4360 A \\
4363 B\end{array}$ \\
\hline \multirow[t]{2}{*}{ Group 5-ECCN } & $\begin{array}{l}1522 \mathrm{~A} \\
4530-\mathrm{B} \text { UF } \\
1564 \mathrm{~A}\end{array}$ \\
\hline & $1565 \mathrm{~A}$ \\
\hline Group 6-ECCN & $\begin{array}{l}3670 \mathrm{~A} \\
36988 \mathrm{~B}\end{array}$ \\
\hline
\end{tabular}

"Neutron generating systems."

Centrifugal balancing machines.

Nuclear power plant related equipment.

Lasers and laser systems.

6 mass spectrometers.

Printed circuit board, substrates, and microcircuits.

Electronic computers.

Lithium enriched in the 6 isotope.

Depleted uranium as shielding.

Contractors who export scientific equipment, nonnuclear materials, and equipment related to nuclear work, and dual-use equipment or technology should review 10 CFR 810,15 CFR 778.3, and 15 CFR 799 before making a shipment. If unable to find the proper entry, the contractor may consult with the Exporters' Service Staff, Bureau of Export Administration, Department of Commerce, Washington, DC, or the Chief of Export Control, DP-323.1, U.S. Department of Energy, Washington, DC 20585.

If the export consists of equipment or a device containing nuclear material, the equipment or device would require a DOC license, while the contained nuclear material would require an NRC license. Exports of nuclear-related technology may require both a DOC license and a DOE 10 CFR 810 non-inimicality determination.

Applications for a DOC license, Form BXA 628P (Figure 28), must be made on Form BXA 622P (Figure 29). It should be filed directly with DOC with a copy to DP-323.1 for advance notice and review. This procedure will provide DP-323.1 some insight into the export activities of the various program offices and assist in directing any concerns or inquiries that may arise from other agencies to the appropriate DOE office. 15 CFR 778, Supplement 1 for "Procedures Established Pursuant to the Nuclear Non-Proliferation Act of 1978," contains procedures for considering the application and resolving disputes. A 1991 amendment to the Act restricts consideration by the Subgroup on Nuclear Export Coordination (SNEC) to 90 days for approval or rejection. See the Forms Supplement, pages 3-35, to the Export Administration Regulations for a facsimile of a completed Form BXA 622P and other BXA or ITA forms that might apply to a particular export. 


\subsection{CANADIAN CUSTOMS DUTIES AND EXCISE TAXES}

Nuclear materials and related equipment shipped to Canada for experimental purposes under a joint agreement for cooperation are exempt from Canadian Customs duties and taxes. Attach the following certification to the Canadian Customs Invoice (Figure 24):

"I hereby certify that the articles or goods herein described are or will become the property of the Government of the United States of America and are to be used solely and exclusively in joint Canada-United States projects or United States Government establishments in Canada and are exempt from Customs duties and excise taxes." In accordance with ORDER-IN-COUNCIL P.C. 1960-1600.

Project or Contract Identification

Exporter

For the U.S. Department of Energy 

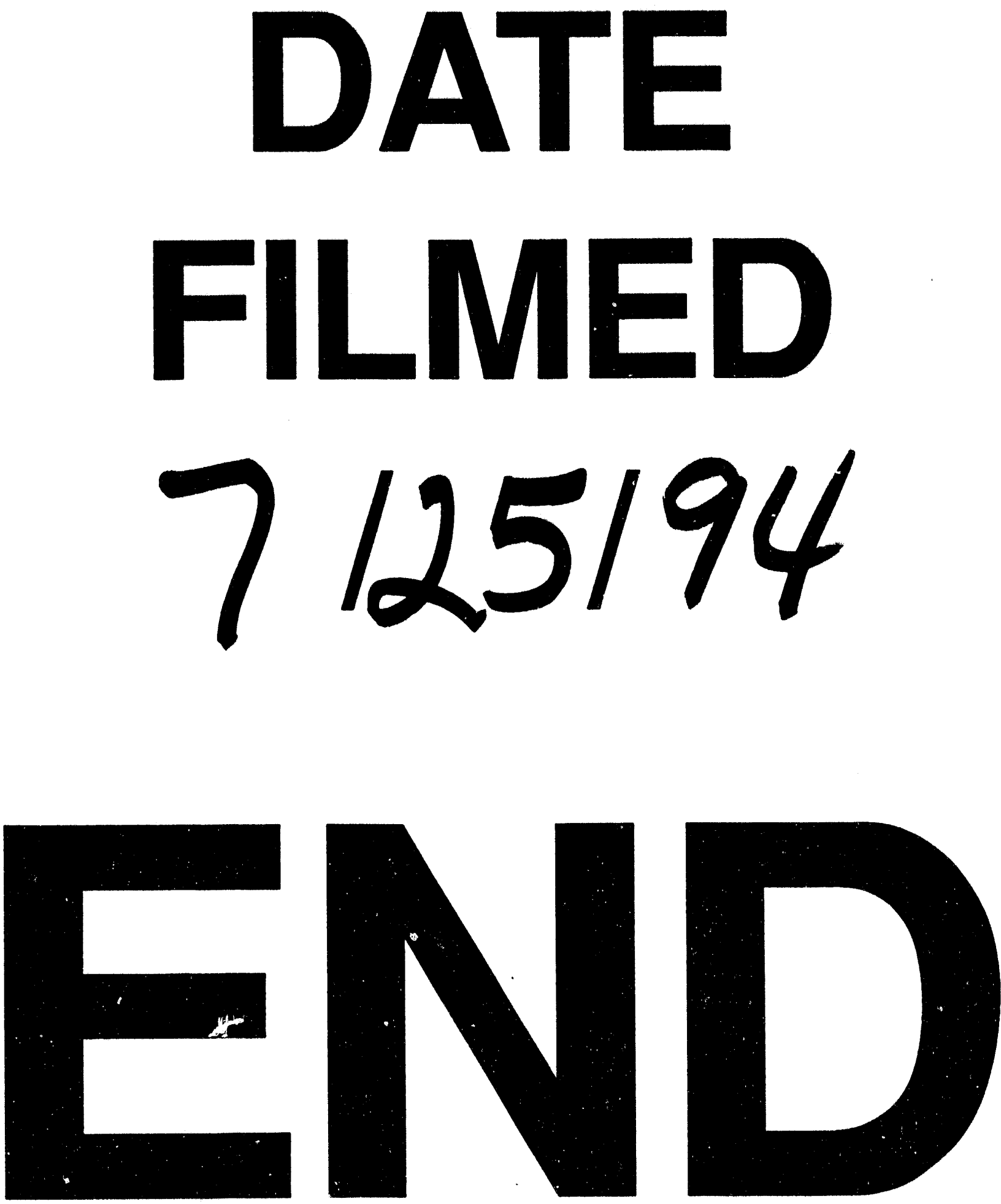